\title{
Beam Tracking Strategies for 5G New Radio Networks Operating in the Millimetre Wave Bands
}

Departamento de Comunicaciones

Universitat Politècnica de València

\author{
A thesis submitted for the degree of \\ Doctor por la Universitat Politècnica de València \\ Valencia, October $7^{\text {th }} 2019$
}

Author:

Carlos Herranz Claveras

Supervisors:

Dr. Jose Francisco Monserrat del Río

Dr. David Martín-Sacristán Gandía 

This dissertation is dedicated to my beloved Anneta and to my grandmother Angela. I love you. 



\section{Abstract}

The arrival of the next generation of mobile communication standards, the so-called Fifth Generation (5G), is already a reality. The first commercial networks have begun to be deployed, and they focus on providing higher data rates. However, the 5G standard goes much further from that and aims at providing support to new services which will revolutionise the society. These new services impose a high level of requirements not only in terms of the data traffic speed, but also in terms of very low latency or incredibly large number of simultaneous connections. This wide variety of requirements cannot be technologically supported by the current Fourth Generation (4G) networks, so it became necessary to move forward with a new paradigm for wireless networks.

With the promise of large amounts of bandwidth, in the order of $\mathrm{GHz}$, the $5 \mathrm{G}$ standard contemplates the use of frequencies in the commonly known as Millimeter Wave (mmWave) band. The mmWave band experiences large propagation losses, which are accentuated in blockage events. Regulatory activities worldwide in the mmWave bands attracted the interest of both the industry and the academia. In the last few years, a tremendous number of contributions on mmWave propagation studies and networks have appeared, most of them based on Multiple-Input Multiple-Output (MIMO) solutions. MIMO architectures allow to beamform, which focuses the radiated energy on certain directions of interest called beams. The additional beam gain compensates the high propagation losses, ensuring the viability of the communications in the mmWave band.

There is an evident lack of viability studies of mmWave MIMO systems in mobile and highly-dynamic environments, where obstacles may block beams and forcing frequent re-configurations. This Thesis work aims to fill this gap from a practical approach and proposes beam management mechanisms utilising the mechanisms and resources offered by the Third Generation Partnership Project (3GPP) 5G radio access standard: 5G New Radio (NR). The practical solutions are based on the efficient use of measurement reports of standardised downlink Reference Signal (RS).

In first place, this Thesis provides a thorough state-of-the-art analysis and corroborates the need of adopting beam tracking solutions for mmWave networks. Then, a complete overview of the $5 \mathrm{G}$ standard mechanisms that enable beam tracking is given. The NR standard does not define a standardised mechanism for beam tracking, leaving the door open to proposals to carry out such monitoring. 
Once the technologies have been identified, the Thesis continues with assessing the impact of the beam tracking strategies on the network and link-level performance. The study is focused on individual point-to-point mmWave links in a realistic urban environment. Based on end-to-end network simulations, the Thesis is interested in assessing the beam tracking success ratio and how beam misalignment affects the perceived Signal to Interference plus Noise Ratio (SINR) and user throughput at pedestrian and vehicular speeds.

The beam tracking solutions proposed in this Thesis fall into two categories. The first category monitors beams based on measuring and reporting beamformed RS. This beam tracking category reaches a maximum beam tracking performance of $98 \%$, with little throughput reduction if the beams of interest are measured with a periodicity below $20 \mathrm{~ms}$. Beam tracking in the second category relies on external information sources. Within this category, this Thesis proposes a fingerprinting database relating beams to the user position and a Machine Learning (ML) model. Fingerprinting beam tracking is technologically viable and provides similar performance levels. However, this solution is very sensitive to errors and requires considering all possible situations. On the other hand, the ML beam tracking model can make predictions with a $9 \%$ of estimation error for the reference data set. 


\section{Resumen}

La llegada de la próxima generación del estándar de comunicaciones móviles, la llamada quinta generación (5G), es prácticamente una realidad. Las primeras redes comerciales han comenzado a ser desplegadas, centrándose en ofrecer altas velocidades de transferencia de datos. Sin embargo, el estándar $5 \mathrm{G}$ va mucho más allá y prevé dar soporte a nuevos servicios que pretenden revolucionar la sociedad. Estos nuevos servicios imponen un nivel alto de requisitos en no solo en cuanto a velocidad del tráfico de datos, sino en cuanto a latencia o número de dispositivos conectados simultáneamente. La amplia variedad de requisitos no puede ser soportada por las redes de cuarta generación (4G), por lo que se hizo necesario plantear un nuevo paradigma para las redes inalámbricas.

Con la promesa de grandes cantidades de ancho de banda sin utilizar, el estándar 5G contempla utilizar frecuencias en la comúnmente conocida como banda de milimétricas (mmWave). Esta banda presenta grandes pérdidas de propagación, que se acentúan si existen bloqueos de señal. Actividades regulatorias del uso de las bandas de milimétricas atrajo el interés tanto de la industria como de la academia en plantear soluciones para dar servicio en estas bandas. En los últimos años se han presentado infinidad de trabajos basados en sistemas con múltiples antenas o MIMO, para conformar las señales transmitidas o recibidas en haces apuntando en determinadas direcciones. La ganancia que aportan los sistemas MIMO pueden compensar las altas pérdidas de propagación, asegurando la viabilidad de las comunicaciones mmWave.

Se ha detectado una evidente falta de estudios sobre la viabilidad de sistemas MIMO en entornos móviles y dinámicos con bloqueos que hagan necesario que el sistema se reconfigure. Esta Tesis pretende cubrir este espacio desde un enfoque práctico y propone mecanismos de gestión de los haces para hacerles un seguimiento utilizando los recursos y mecanismos del nuevo estándar 5G. Las soluciones aportadas se basan en el uso eficiente de los reportes de medidas de las señales de referencia estandarizadas en enlace descendente.

En primer lugar, esta Tesis recoge un análisis minucioso del estado del arte, donde se corrobora la necesidad de aportar soluciones de seguimiento de haces en sistemas de comunicaciones en la banda de milimétricas. Además, se estudian los diferentes mecanismos definidos en el estándar $5 \mathrm{G}$ y que posibilitan el seguimiento. Cabe destacar que el estándar no define un mecanismo único a seguir, permitiendo presentar propuestas.

Una vez conocidas las tecnologías, se centra el estudio en el impacto del seguimiento sobre las prestaciones a nivel de red y de enlace. Dicho estudio se 
realiza sobre un sistema punto a punto, donde el terminal móvil se desplaza por un entorno urbano. En base a simulaciones de red, se cuantifica el índice de seguimiento de haz y de cómo dicho seguimiento afecta a la relación señal a ruido más interferencia (SINR) y la tasa de transmisión del usuario.

Las soluciones de seguimiento propuestas en esta Tesis se pueden clasificar en dos categorías. En una primera categoría, se realiza el seguimiento en base a reportes de medidas de las señales de referencia. Se llega a alcanzar un seguimiento del $98 \%$ con poca penalización en la tasa de transmisión si se monitorizan los haces de interés con una periodicidad menor de $20 \mathrm{~ms}$. En la segunda categoría caben mecanismos de seguimiento que hacen uso de fuentes externas de información. Dentro de esta categoría, se propone un fingerprinting que relacione haces con la localización reportada y un modelo de machine learning (ML) que prediga los haces a utilizar. El fingerprinting proporciona los mismos niveles de rendimiento. Sin embargo, esta solución es muy sensible a errores y requiere considerar todos los casos posibles, lo que la hace tecnológicamente muy costosa de implementar. En cambio, el modelo de ML, que hace predicciones con un error del $9 \%$, presenta una alternativa más factible. 


\section{Resum}

L'arribada de la següent generació de l'estàndard de comunicacions mòbils, l'anomenada cinquena generació $(5 \mathrm{G})$, es pràcticament una realitat. Les primeres xarxes comercials han començat a desplegar-se i s'han centrat en oferir altes velocitats de transferència de dades. No obstant, l'estàndard $5 \mathrm{G}$ va molt més allà y preveu donar suport a nous serveis que pretenen revolucionar la societat. Estos nous serveis imposen un alt nivell de requisits no sols en quant a velocitat de tràfic de dades, si no també en quant a latència o número de connexions simultànies. L'ampla varietat de requisits no es suportada per les xarxes de quarta generació $(4 \mathrm{G})$ actuals, per el qual es va fer necessari un nou paradigma de xarxes sense fil.

Amb la promesa de amplies quantitats d'ample de banda, l'estàndard 5G contempla utilitzar freqüències a la banda de mil-limètriques. Esta banda presenta l'inconvenient d'experimentar grans pèrdues de propagació, que s'accentuen en cas de bloqueigs. L'apertura de les bandes de mil-limètriques va atraure l'interès tant de l'industria com de l'acadèmia en plantejar solucions per a donar servei en estes bandes. En els últims anys s'han presentat infinitat de treballs basats en sistemes amb múltiples antenes o MIMO, per a conformar els senyals transmesos o rebuts en feixos apuntant en determinades direccions d'interès. El guany de feix es pot utilitzar per a compensar les pèrdues de propagació, assegurant la viabilitat de les comunicacions en la banda de mil.limètriques.

No obstant això, s'ha detectat una preocupant manca d'estudis sobre la viabilitat d'estos sistemes en entorns mòbils i dinàmics, amb obstacles que bloquejen els feixos i facen necessari que el sistema es reconfigure. El present treball de Tesi pretén cobrir este espai buit i des d'un punt de vista pràctic, es proposen mecanismes de gestió dels feixos per a ser el seguiment utilitzant els recursos i mecanismes dels que disposa l'estàndard 5G. D'esta manera, les solucions aportades es basen en la utilització eficient dels reports de mesures dels senyals de referència del enllaç descendent.

En primer lloc, esta Tesi recull una anàlisi minuciosa de l'estat de l'art on es corrobora la necessitat de aportar solucions de seguiment de feixos per a comunicacions en la banda de freqüències mil-limètriques. A més a més, s'estudien els diferents mecanismes definits a l'estàndard $5 \mathrm{G}$ i que possibiliten el seguiment. Cap destacar que l'estàndard no defineix un mecanisme únic, si no que deixa la porta oberta a presentar propostes. 
Una vegada conegudes les tecnologies, l'estudi es centra en l'impacte del seguiment sobre les prestacions a nivell de xarxa i d'enllaç. Este estudi es realitza sobre un sistema MIMO punt a punt, en una única estació base i un terminal mòbil desplaçant-se en un entorn urbà. En base a simulacions d'extrem a extrem, es quantifica l'índex de seguiment de feix i com l'anomenat seguiment afecta a la relació senyal a soroll més interferència (SINR) i a la taxa instantània de transmissió de l'usuari.

Les solucions de seguiment de feixos propostes a la Tesi es poden classificar en dos categories. A la primera categoria, el seguiment de feixos es realitza en base als reports de mesures dels senyals de referència. Es pot arribar a una taxa de seguiment del $98 \%$ amb poca penalització de la taxa de transmissió si els feixos d'interès es mesuren amb una periodicitat menor a $20 \mathrm{~ms}$. A la segona categoria pertanyen els algoritmes que utilitzen fonts d'informació externes. Dins d'aquesta categoria es proposa un fingerprinting que relaciona un parell de feixos amb la ubicació de l'usuari, i a banda un model d'intel-ligència artificial (IA) que preveu el feix a utilitzar. El fingerprinting ofereix el mateix rendiment. Però, esta solució es molt sensible a errors i requerix considerar tots els casos possibles, fent-la tecnològicament molt costosa. En canvi, el model IA, que fa prediccions amb un error del $9 \%$, es una alternativa més realista. 


\section{Acknowledgements}

I write these lines to express my gratitude to all the people who helped me to accomplish this Thesis. First and foremost, I would like to thank my supervisor, Professor Jose F. Monserrat, and Professor Narcís Cardona for the great opportunity of conducting my research activities in the Mobile Communications Group with its great team of scientists. I really appreciate the excellent opportunities they offered me to grow personally and professionally. I also want to thank my supervisor Dr. David Martín-Sacristán for his valuable help in many scientific issues and for many fruitful technical discussions and guidance. I would like to thank again Professor Jose F. Monserrat for his guidance and for facilitating all the problems encountered during all the stages of writing this Thesis.

I would also like to thank Dr. Sundeep Rangan and, specially, Dr. Marco Mezzavilla for inviting me joining their research group at the NYU Wireless. I spent a great time with you during my research stay and I obtained a very valuable knowledge. I also want to thank all the staff and great group of researchers there, specially to Kiren for dealing with all the official documents with the American government. I want to thank Professor Dennis Shasha for hosting the joyful Tuesday lunches with very interesting people. Thank you all for giving me the opportunity to live the American dream.

I would also like to thank all the people at the Department of Signal Theory and Communications at the Universitat Politècnica de CatalunyaBarcelonaTech. Thank you, Sílvia, Joan, David, Jose Luís, Rolando and Lalo for welcoming me in your research group. I want to give special thanks to Professor Mario García-Lozano for his valuable advice and guidance during the last part of the Thesis.

I would like to acknowledge to all my colleagues at iTEAM for making my time at the research institute more enjoyable. Special mention to Pepe, Guillermo, Jorge, Irene, Alicia, Sonia, Pablo, Miguel, Jordi Joan, David Garcia, Jordi, Tere, Sandra, Dani, Gerardo, Carlos, Manuel, Edu, Alex, Sergio, Martina, Sofía, Conchi, Danaisy, Saúl, Josué, Samuel, Dani, Toni and Nora.

I want to thank the Spanish Ministry of Education and Professional Formation for funding this Thesis work with an official pre-doctoral contract grant.

I want to express my gratitude to Dr. David López-Pérez, Dr. David Gutiérrez and Dr. Lorenza Giupponi for reviewing this Thesis. I also want to thank Dr. David Gómez-Barquero, Dr. María José Domènech and Dr. Jordi Joan Giménez por accepting taking part of the Thesis tribunal. 
I would like to give specials thank my girlfriend Anna. Thank you for your infinite love, patience, care and for believing in me. You have always been there for me. I would also thank to my little dog One for always lying next to me during the endless days spent writing this Thesis manuscript.

Finally, I want to thank my relatives and closest friends for believing in me. Thanks to my parents, Juan and Isabel, and my brother Ivan for their support. Thanks to my grandmothers Angela and Isabel and to my grandfather Fermín for their unconditional love and support. Thanks to Isabel Alvado for caring so much. Special thanks to my friends, who were of great support in providing the necessary happy distraction to rest my mind outside of my research.

Carlos 




\section{Table of contents}

Acronyms $\quad$ v

Notation $\quad$ xiii

1 Introduction $\quad \mathbf{1}$

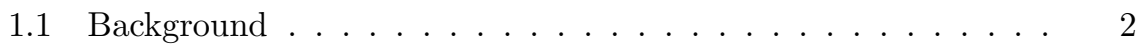

1.1 .1 Why $5 \mathrm{G} ? \ldots \ldots \ldots \ldots 2$

1.1 .2 3GPP Releases . . . . . . . . . . . . . . 8

1.1.3 Multi-antenna transmission in 3GPP . . . . . . . . 9

1.1.4 Channel models for simulations . . . . . . . . . . . . 12

1.2 State of the art analysis . . . . . . . . . . . . . . . . . 13

1.2.1 Millimeter Wave communications . . . . . . . . . 13

1.2 .2 Beamforming ....................... 18

1.2.3 Beam tracking solutions . . . . . . . . . . . 25

1.3 Problem formulation and Thesis scope . . . . . . . . . . . . . . 31

1.4 Thesis outline . . . . . . . . . . . . . . . 33

1.5 Publications . . . . . . . . . . . . . . . . 34

$2 \quad 3 G P P$ NR Release $15 \quad 37$

2.1 Physical layer aspects . . . . . . . . . . . . . . . 37

2.1.1 Flexible radio frame structure . . . . . . . . . . . 38

2.1.2 Synchronisation and initial access in NR . . . . . . . . . 41

2.1.3 Channel State Information in NR . . . . . . . . . . . . 44

2.1.4 Downlink signals and overhead . . . . . . . . . . 46

2.2 Higher layer aspects . . . . . . . . . . . . . . . . . . . . . . . 49

2.2.1 Beam management . . . . . . . . . . . . 49

2.2.2 Beam measurement and reporting . . . . . . . . . 52

2.2 .3 UE positioning . . . . . . . . . . . . . . . 56

2.3 Conclusions . . . . . . . . . . . . . . . 56 
3 System Modelling $\quad 59$

3.1 Network architecture . . . . . . . . . . . . . . . . 60

3.2 Channel models for mmWave . . . . . . . . . . . . . 63

3.2.1 3GPP channel model for frequencies over $6 \mathrm{GHz} \ldots .63$

3.2 .2 Ray tracing tool in Unity . . . . . . . . . . . . . . 66

3.3 Codebook-based beamforming . . . . . . . . . . . . . 71

3.4 Conclusions . . . . . . . . . . . . . . . . . 75

4 Simulation Framework and Visualisation $\quad 77$

4.1 End-to-end network simulation with ns-3 . . . . . . . . 78

4.1.1 The mmWave module . . . . . . . . . . . . . 78

4.2 Extension of the mmWave module . . . . . . . . . . . 79

$4.2 .1 \quad$ Flexible radio frame . . . . . . . . . . . . . . 79

4.2 .2 Beam management . . . . . . . . . . . . . . . . 80

4.3 Beam management framework validation . . . . . . . . . . 88

4.3.1 Circular walk . . . . . . . . . . . . . . . . 88

4.3 .2 Linear walk . . . . . . . . . . . . . . . . . . . . . . 92

4.4 Visualisation . . . . . . . . . . . . . . . . 95

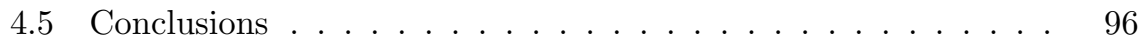

5 Beam Tracking Techniques $\quad 99$

5.1 Simulation setup . . . . . . . . . . . . . . . . . 100

5.1 .1 Simulated scenario . . . . . . . . . . . . . . . 100

5.1 .2 Tracking performance indicators . . . . . . . . . 107

5.1 .3 Baseline case definition . . . . . . . . . . . . . . . . 109

5.2 Tracking with SSB . . . . . . . . . . . . . . . 110

5.2 .1 General considerations . . . . . . . . . . . . . . 110

5.2 .2 Urban macro scenario . . . . . . . . . . . . . . . . 111

5.2 .3 Urban micro scenario . . . . . . . . . . . . . . . . . 119

5.3 Tracking with SSB and CSI-RS . . . . . . . . . . . . 128

5.3 .1 Beam tracking strategies . . . . . . . . . . . . . . 128

5.3.2 Urban macro scenario . . . . . . . . . . . . . . . . 131

5.3 .3 Urban micro scenario . . . . . . . . . . . . . . . . . 142

5.4 Rotation . . . . . . . . . . . . . . . . . . 153

5.4 .1 Coordinate system . . . . . . . . . . . . . . 153

5.4 .2 Analytical study on beam prints . . . . . . . . . 153

5.4 .3 UE rotation in walk . . . . . . . . . . . . . . . 158

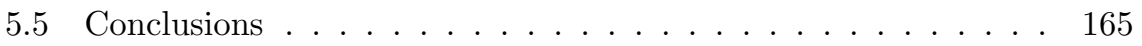


6 Contextual Beam Tracking Techniques 169

6.1 Inverse fingerprinting for beam tracking . . . . . . . . 170

6.1 .1 Mechanism description . . . . . . . . . . . . . . 170

6.1.2 System model assumptions . . . . . . . . . . . . . . 171

6.1.3 Simulation results with error-free measurements . . . . 177

6.1.4 Simulation results with erroneous measurements . . . . 188

6.1.5 Beam pair determination based on user location . . . . 194

6.1.6 Conclusions on fingerprinting . . . . . . . . . . . . 196

6.2 Beam tracking with machine learning . . . . . . . . . . . . . . 198

6.2.1 Data preprocessing . . . . . . . . . . . . . . . 199

6.2.2 The Multi-Layer Perceptron (MLP) _ . . . . . . . . 200

6.2.3 An MLP model for beam tracking . . . . . . . . . . 203

6.2.4 Conclusions on machine learning applications for beam tracking . . . . . . . . . . . . . . . 209

7 Conclusions and Future Research $\quad 211$

7.1 Concluding remarks . . . . . . . . . . . . . . 211

7.2 Future research directions . . . . . . . . . . . . . . . . 214

Appendices $\quad 217$

A Visualisation platform . . . . . . . . . . . . 217

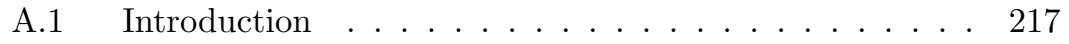

A.2 $5 \mathrm{G}$ visualisation $\ldots \ldots \ldots \ldots \ldots \ldots \ldots$

A.3 Open source graphical environment . . . . . . . . . . 219

A.4 Interaction capabilities . . . . . . . . . . . . . 225

A.5 Lessons learned . . . . . . . . . . . . . . . . . 226

A.6 Conclusions . . . . . . . . . . . . . . . . 227

$\begin{array}{ll}\text { References } & 229\end{array}$ 



\section{Acronyms}

2D

3D

3GPP

4G

$5 \mathrm{G}$

$5 \mathrm{GC}$

$6 \mathrm{G}$

ADC

AI

AoA

AoD

AR

BGL

BS

BWP

CDM

CNN

CoMP
Two Dimension

Three Dimension

Third Generation Partnership Project

Fourth Generation

Fifth Generation

5G Core Network

Sixth Generation

Analog-to-Digital Converter

Artificial Intelligence

Angle of Arrival

Angle of Departure

Augmented Reality

Beam Gain Loss

Base Station

Bandwidth Part

Code Division Multiplexing

Convolutional Neural Network (NN)

Coordinated Multi-Point 
CP

CQI

CRI

CRS

CSI

CSI-RS

CW

cmWave

DCI

DFT

DL

DM-RS

DoF

DS

eMBB

eNodeB

EPC

FDD

FDM

FD-MIMO

FR

FR1

FR2

FSPL
Cyclic Prefix

Channel Quality Indicator

Channel State Information Reference Signals (CSI-RS)

Resource Indicator

Channel state information Reference Signal

Channel State Information

Channel State Information Reference Signals

Congestion Window

Centimetre Wave

Downlink Control Information

Discrete Fourier Transform

Downlink

Demodulation Reference Signal

Degrees of Freedom

Delay Spread

Enhanced Mobile Broadband

evolved Node B

Evolved Packet Core

Frequency Division Duplexing

Frequency Division Multiplexing

Full Dimension MIMO

Frequency Range

Frequency Range (FR) 1

FR 2

Free Space Power Loss 


$\begin{array}{ll}\text { GIS } & \text { Geographical Information Service } \\ \text { gNB } & \text { NR base station } \\ \text { GNSS } & \text { Global Navigation Satellite System } \\ \text { GPS } & \text { Global Positioning System } \\ \text { GPGPU } & \text { General-Purpose Graphics Processing Unit } \\ \text { GSM } & \text { Global System for Mobile Communication } \\ \text { GUI } & \text { Graphical User Interface (UI) } \\ \text { HPBW } & \text { Half-Power Beamwidth } \\ \text { IEEE } & \text { Institute of Electrical and Electronic Engineers } \\ \text { IMT } & \text { International Mobile Telecommunication } \\ \text { IMT-2020 } & \text { International Mobile Telecommunication (IMT) 2020 } \\ \text { IoT } & \text { Internet of Things } \\ \text { ISD } & \text { Inter-Site Distance } \\ \text { ITU } & \text { International Telecommunication Union } \\ \text { ITU-R } & \text { International Telecommunication Union (ITU) } \\ \text { KPC } & \text { Radiocommunication Sector } \\ \text { KPI } & \text { Kronecker Product Codebook } \\ \text { LAN } & \text { Key Performance Indicator } \\ \text { LI } & \text { Local Area Network } \\ \text { LoS } & \text { Layer Indication } \\ \text { LTE } & \text { Line of Sight } \\ \text { Long Term Evolution }\end{array}$

LTE-Advanced Long Term Evolution (LTE) Advanced

L1-RSRP Layer 1 Reference Signal Received Power (RSRP)

MAC Medium Access Control 


$\begin{array}{ll}\text { MAC CE } & \text { Medium Access Control (MAC) Control Element } \\ \text { MIB } & \text { Master Information Block } \\ \text { MIMO } & \text { Multiple-Input Multiple-Output } \\ \text { ML } & \text { Machine Learning } \\ \text { MLP } & \text { Multi-Layer Perceptron } \\ \text { MMSE } & \text { Minimum Mean Square Error } \\ \text { MO } & \text { Measurement Object } \\ \text { MTC } & \text { Machine-Type Communications } \\ \text { mMTC } & \text { Massive Machine-Type Communications (MTC) } \\ \text { MU-MIMO } & \text { Multi-User MIMO } \\ \text { mmWave } & \text { Millimeter Wave } \\ \text { NLoS } & \text { Non Line of Sight } \\ \text { NN } & \text { Neural Network } \\ \text { NR } & \text { New Radio } \\ \text { NSA } & \text { Non Standalone } \\ \text { OFDM } & \text { Orthogonal Frequency Division Multiplexing } \\ \text { OSM } & \text { Open Street Map } \\ \text { PBCH } & \text { Physical Broadcast Channel } \\ \text { PDP } & \text { Packet Data Protocol } \\ \text { PDSCH } & \text { Physical Downlink Shared Channel } \\ \text { PHY } & \text { Physical } \\ \text { PMI } & \text { Precoding Matrix Indicator } \\ \text { PRACH } & \text { Physical Random Access Channel } \\ \text { PSD } & \text { Power Spectral Density } \\ \text { PSS } & \text { Primary Synchronization Signal } \\ & \end{array}$




$\begin{array}{ll}\text { PUCCH } & \text { Physical Uplink Control Channel } \\ \text { PUSCH } & \text { Physical Uplink Shared Channel } \\ \text { QoS } & \text { Quality of Service } \\ \text { RACH } & \text { Random Access Channel } \\ \text { RAN } & \text { Radio Access Network } \\ \text { RAT } & \text { Radio Access Technology } \\ \text { RB } & \text { Resource Block } \\ \text { RE } & \text { Resource Element } \\ \text { ReLU } & \text { Rectifier Linear Unit } \\ \text { RF } & \text { Radio Frequency } \\ \text { RI } & \text { Rank Indicator } \\ \text { RLF } & \text { Radio Link Failure } \\ \text { RMa } & \text { Rural Macro-cell } \\ \text { RMSE } & \text { Root Mean Squared Error } \\ \text { RNN } & \text { Recurrent NN } \\ \text { RRC } & \text { Radio Resource Control } \\ \text { RS } & \text { Reference Signal } \\ \text { RSRP } & \text { Reference Signal Received Power } \\ \text { RTT } & \text { Round Trip Time } \\ \text { SA } & \text { Standalone } \\ \text { SCS } & \text { Sub-Carrier Spacing } \\ \text { SIB } & \text { System Information Block } \\ \text { SIB1 } & \text { System Information Block (SIB) 1 } \\ \text { SINR } & \text { Signal to Interference plus Noise Ratio } \\ \text { SISO } & \end{array}$


SMTC Synchronization Signal (SS)/Physical Broadcast

Channel (PBCH)-Block (SSB)-based Measurement Timing

Configuration

SNR Signal to Noise Ratio

SS Synchronization Signal

SSB SS/PBCH-Block

SSS Secondary Synchronization Signal

SU-MIMO Single User MIMO

SVM Support Vector Machine

TCP Transmission Control Protocol

TDD Time Division Duplexing

TDM Time Division Multiplexing

TR Technical Report

TRP Transmit Receive Point

TXRU Transceiver Architecture

UE User Equipment

UI User Interface

UL Uplink

ULA Uniform Linear Array

UMa Urban Macro-cell

UMi Urban Micro-cell

UML Unified Modeling Language

URA Uniform Rectangular Array

URLLC Ultra-Reliable Low-Latency Communications

UT User Terminal

V2V Vehicle-to-Vehicle 
V2X

VP

VR

WG

WI

WiMAX

Wi-Fi

XML
Vehicle-to-Everything

Visualisation Platform

Virtual Reality

Working Group

Work Item

Worldwide Interoperability for Microwave Access

Wireless Fidelity

Extensible Markup Language 



\section{Notation}

This section describes the notation used in this Thesis.

$\mathbf{A}$ (boldface capital letter) and $\mathbf{A}_{k}$ represent matrices.

$[\mathbf{A}]_{i j}$ is the element of matrix $\mathbf{A}$ in row $i$ and column $j$.

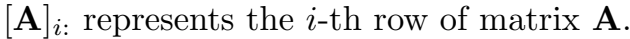

$[\mathbf{A}]_{: j}$ represents the $j$-th column of matrix $\mathbf{A}$.

$\mathbf{A}^{H}$ denotes the conjugate transpose or Hermitian transpose of $\mathbf{A}$.

$\mathbf{I}_{n}$ denotes the identity matrix of size $n$.

$\mathbf{a}$ (boldface lower-case letter) and $\mathbf{a}_{k}$ represent vectors.

$[\mathbf{a}]_{i}$ represents the $i$-th element of vector $\mathbf{a}$.

$a$ and $A$ (not boldface italic letters) represent scalars.

$\mathcal{A}$ (calligraphic letter) represents a set of numbers.

$|\mathcal{A}|$ denotes the number of elements in the set $\mathcal{A}$. 



\section{Chapter 1}

\section{Introduction}

The concept of mobile network is evolving. The next generation of the mobile communication standard, the so-called Fifth Generation (5G), will bring profound modifications in many aspects. The projected roadmap to the ubiquitous high-speed network promised by the year 2020 implies managing ultra-dense Multi-User MIMO (MU-MIMO) cellular deployments. On the other hand, the use of additional frequency bands in the Millimeter Wave (mmWave) domain is presented as a major requirement in order to increase the network capacity 1000-fold, given the complete saturation of the electromagnetic spectrum in frequencies below $6 \mathrm{GHz}$, in which legacy wireless networks operate.

The present chapter of the Thesis introduces the results of the research activity conducted on the field of link adaptation mechanisms for massive Multiple-Input Multiple-Output (MIMO) 5G wireless networks operating in frequencies over $6 \mathrm{GHz}$, the called Centimetre Wave (cmWave) and mmWave bands. This chapter serves as an introduction to the Thesis and explains the motivation of the work done and provides the necessary technological background. The background is structured as a series of the explanations of some fundamental concepts or by presenting the most significant references.

With this aim, this chapter has been divided into the following sections:

- Section 1.1 situates the Thesis in its technological and historical context.

- Section 1.2 analyses the state of the art in the field covered by this Thesis previous to the beginning of this Thesis work. Concurrent research activities are also commented.

- Section 1.3 identifies the main problems discussed by the research community concerning the specific field covered in this Thesis. The section 


\section{CHAPTER 1. INTRODUCTION}

concludes establishing the research hypothesis and the research objectives of the Thesis.

- Section 1.4 outlines the structure of the Thesis summarising the main contents of each chapter.

- Section 1.5 lists the publications related with this Thesis, including scientific papers in journals and conferences.

\subsection{Background}

This section presents the context in which the Thesis has been undertaken. This process includes the evolution process of the mobile and wireless communication networks to cope with the voracious demand of user data traffic and the standardised solutions adopted through the years.

\subsubsection{Why 5G?}

Mobile communications have fueled the evolution of society and technology for the past three decades. Since the irruption of the smartphones, communication devices are becoming more and more frequent in different situations of people's daily lives. This trend is expected to continue, making technology and society bounds more profound.

The future will impose more exigent requirements to the wireless networks. New services and applications will demand higher volumes of data traffic from a massive number of devices simultaneously connected, each with different service requirements and hardware complexities. The creation of new applications will open up new revenue streams for mobile operators, which are also pushed to reduce their operation expenditure and maintain user tariffs low.

The services to come and their requirements will undoubtedly need of innovative solutions. In this scenario, the International Telecommunication Union (ITU) Radiocommunication Sector (ITU-R) is pushing the wireless technologyenablers to a revolutionary new network paradigm where new scenarios and services will flourish. With the firm intention to conclude in the year 2020, the new International Mobile Telecommunication (IMT) 2020 (IMT-2020) specification [1] for the future generation of wireless communications focuses on three main usage scenarios: Enhanced Mobile Broadband (eMBB), Massive Machine-Type Communications (MTC) (mMTC) and Ultra-Reliable Low-Latency Communications (URLLC). The expected capacities of the $5 \mathrm{G}$ network are listed in Table 1.1 
Table 1.1: Wireless network requirement for IMT-2020.

\begin{tabular}{|c|c|c|}
\hline Capability & Target value & Usage scenario \\
\hline \hline Peak data rate & $20 \mathrm{Gbps}$ & eMBB \\
\hline Guaranteed data rate & $100 \mathrm{Mbps}$ & eMBB \\
\hline Latency & $1 \mathrm{~ms}$ & URLLC \\
\hline Maximum user speed & $500 \mathrm{Km} / \mathrm{h}$ & eMBB/URLLC \\
\hline Simultaneous connections & $10^{6} / \mathrm{km}^{2}$ & mMTC \\
\hline Energy efficiency & Equal to $4 \mathrm{G}$ & eMBB \\
\hline Area traffic capacity & $10 \mathrm{Mbps} / \mathrm{m}^{2}$ & eMBB \\
\hline Spectrum efficiency & $(3-4) \mathrm{x} 4 \mathrm{G}$ & eMBB \\
\hline
\end{tabular}

Achieving the requirements imposed by IMT-2020 will require the adoption of innovative solutions in the network. The enhancements in data rate and network capacity can be tacked down from different perspectives. One of them is increasing the spectral efficiency of the network. To that aim, advanced physical layer techniques can be introduced, including advanced modulation and coding schemes. Advanced processing techniques like Three Dimension (3D) beamforming or using massive MIMO are also promising solutions to increase the spectral efficiency by performing what is called spatial multiplexing.

A revolutionary novelty in IMT-2020 is that it opened the door, for the first time, to consider carrier frequencies between 6 and $100 \mathrm{GHz}$ for the $5 \mathrm{G}$ radio access. Rather than suggesting a common range of frequencies for all the geographic areas, the IMT-2020 recommends policy regulators of the different regions to make and effort and harmonise the use of frequency bands. Harmonisation will simplify the network equipment and terminals since they will equip a single radio front-end. Such complexity reduction will also help reduce the energy consumption, thus extending the battery life of the devices, and facilitate the economies of scale. Furthermore, the IMT-2020 recommends the new frequency bands to have large and contiguous portions of spectrum. The bandwidth must be flexible and long enough to accommodate the IMT-2020 usage cases may require bandwidths from a few $\mathrm{MHz}$ to $1 \mathrm{GHz}$ or even more.

\section{GPP 5G timeline}

It is clear now that some of the new requirements imposed by the IMT-2020 specification are incompatible with the current Long Term Evolution (LTE) technology. In order to fulfil the IMT requirements, Third Generation Partnership Project (3GPP) created the next $\mathrm{G}$ of its mobile network standards and named it New Radio (NR) [2]. 


\section{CHAPTER 1. INTRODUCTION}

It was during TSG \#67 in March 2015 when 3GPP reported its tentative timeline for the development of their 5G standard [3]. Later, during RAN \#69 in September 2015, 3GPP RAN group identified three initial steps which were necessary to support the development of NR: 1) preparation of a new channel model supporting larger frequencies; 2) study the new scenarios for $5 \mathrm{G}$; and 3) define RAN Working Group (WG) to evaluate technology solutions for 5G. The timeline of the development of each of the three requirements started with the creation of their individual Release 14 Study Items (SI) in successive RAN meetings is the following.

The first SI, which also appeared in RAN \#69, was the Release 14 SI entitled "Study on channel model for frequency spectrum above $6 \mathrm{GHz}$ " [4]. This study centred on channel models for frequencies above $6 \mathrm{GHz}$. The study concluded with the creation of the $3 \mathrm{GPP}$ standard model defined in Technical Report Technical Report (TR) 38.900, which was created in RAN \#72 meeting held in June 2016 [5]. This TR can be seen as the first official Release 15 document centred in NR. Later, the channel model for frequency spectrum above $6 \mathrm{GHz}$ was integrated with the channel model for sub- $6 \mathrm{GHz}$ frequencies in TR 38.901 [6].

The Release 14 SI "Study on Scenarios and Requirements for Next Generation Access Technologies" [7] was initialised in RAN \#70 in December 2015. This study spanned for one year until RAN \#74 meeting in December 2016, when 3GPP moved the SI to the standard document TR 38.913 [8].

In March 2016, ITU-R invited for candidate radio interface technologies for IMT-2020 in a Circular Letter. The overall objectives of IMT-2020 were set via ITU-R M.2083 and the requirements were provided in ITU-R M.2410 [9].

In alignment with the ITU-R Circular Letter, 3GPP started during RAN \#71 meeting in March 2016, the Rel-14 SI "Study on New Radio (NR) Access Technology" [10] with the purpose to identify the usage cases and their requirements. This study completed at RAN \#75 in March 17 and it is part of the 3GPP TR 38.912 [11]. This standard document summarises the features of the new radio technologies, extending those contained in [8], and provides feasibility and capacity studies.

The outcome of RAN \#75 meeting in March 17 was an important milestone in the development of NR. 3GPP started a Release 15 a Work Item (WI) on "New Radio Access Technology" [12]. In this meeting, the RAN work groups committed to accelerate the developing and deployment of NR. Due to the complexity of the $5 \mathrm{G}$ network and the very challenging timing, the NR release plan was split into 3 phases addressing different network operator demands.

For this aim, 3GPP released an "early Release 15 drop", which was intended for speeding the adoption of $5 \mathrm{G}$. This architectural solution consisted in integrating $\mathrm{NR}$ base stations (called NR base station (gNB)) to an existing 


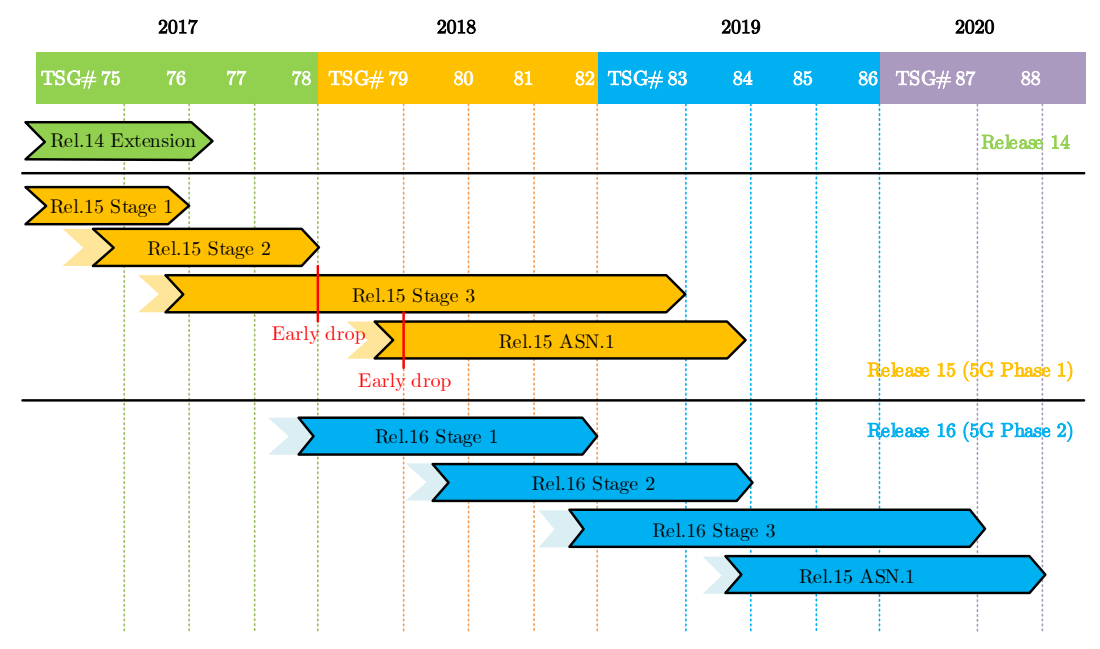

Figure 1.1: 3GPP roadmap for 5G [13]

LTE Advanced (LTE-Advanced) network in such a way that NR only conveys user-plane data and all the control signalling is conveyed throughout LTE's legacy Evolved Packet Core (EPC). 3GPP refers to this architectural solution as NR Non Standalone (NSA) mode.

3GPP contemplates a "regular Release 15 freeze" release, which focus on the NR Standalone (SA) mode. Opposite to the NSA mode, this architectural option conveys the signalling data through an 5G Core Network (5GC), not requiring the background support of an LTE network.

Finally, there is a third release of NR named "late Release 15 drop", which provides inter-networking flexibility between LTE and NR networks.

3GPP has been actively developing 5G specifications since June 2017 when LTE Release 14 was declared to be completed (freeze). 3GPP planned the full development of the NR standard in two phases and they spanned throughout Releases 15 and 16. Completion of Release 15 is referred to as 5G Phase 1, and the $5 \mathrm{G}$ Phase 2 occurs throughout the completion of Release 16 . The whole roadmap to $5 \mathrm{G}$ is illustrated in 1.1 , where the "Stage 1 " refers to the service description, "Stage 2" described the architectural and logical analysis of the network, and "Stage 3" is the implementation of the protocols. 


\section{CHAPTER 1. INTRODUCTION}

The first set of $3 \mathrm{GPP} 5 \mathrm{G}$ specification documents, NR in NSA mode saw the light in December 2017. The SA mode was due to by September 2018 but was concluded in June 2018.

The first commercial call using the NSA 5G NR standard was completed in February 2018 on a test network in Spain, by Vodafone and Huawei. Vodafone started to commercialise $5 \mathrm{G}$ coverage in 15 cities in the Spanish territory in June 2019 .

\section{New operation frequencies}

As already stated, the services envisioned for $5 \mathrm{G}$ will require a large number of contiguous chunks of spectrum, way beyond the currently available bandwidths in legacy cellular systems. This basic requirement made mmWave bands so attractive for $5 \mathrm{G}$ systems, given the vast unoccupied spectrum.

The IMT-2020 system requirements demand at least $100 \mathrm{MHz}$ width channels, extending this compromise to up to $1 \mathrm{GHz}$ for frequencies in the mmWave band.

3GPP, following the IMT-2020 guidelines on use of the 5G spectrum, extended the operation frequencies used in legacy cellular systems to higher frequencies for NR. Accordingly, 3GPP separates the $5 \mathrm{G}$ spectrum in two Frequency Range (FR) $[2,14]$. The FR 1 (FR1) term refers to frequencies below $6 \mathrm{GHz}$, and the FR 2 (FR2) term refers to the inaccurately called mmWave band. More precisely, FR2 includes operation frequencies ranging from 24.25 to $52.60 \mathrm{GHz}$.

One of the mandatory objectives in IMT-2020, and extended to 3GPP NR, is the spectrum harmonisation among geographical areas. This implies that regions must make an effort to make available the frequency bands identified for $5 \mathrm{G}$ deployments. Spectrum harmonisation is desirable because it allows using the same mobile terminals and radio access network equipment worldwide. This brings significant benefits in terms of end-user and network equipment simplification, and cost manufacturing cost reduction with the introduction of economies of scale. An harmonised spectrum is even more desirable in mmWave frequencies. Having a unified ecosystem eases the development of advanced multi-antenna techniques.

3GPP TR 38.815 is devoted to the spectrum harmonisation efforts for mmWave [15]. The document summarises the initiatives taken by governments of The United States of America, the European Union, South Korea, Japan, and China, with respect to their spectrum licensing activities for 5G. The original tentative frequency bands for each region are illustrated in Figure 1.2.

On July 2016, the USA Federal Communications Commission (FCC) open up nearly $11 \mathrm{GHz}$ of spectrum in the mmWave band for commercial wireless 


\subsection{Background}

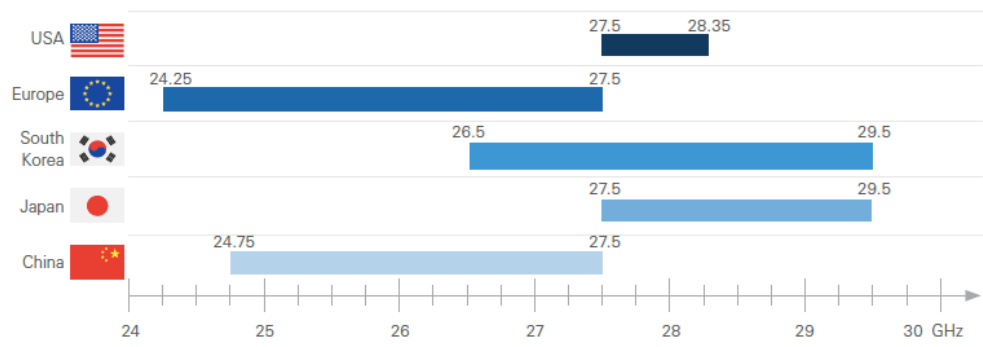

Figure 1.2: The plans of spectrum use for $5 \mathrm{G}$ systems proposed in different regions are harmonised in the $24-30 \mathrm{GHz}$ band [15].

services: $3.85 \mathrm{GHz}$ of licensed spectrum and $7 \mathrm{GHz}$ of unlicensed spectrum by creating a new Upper Microwave Flexible Use service (UMFUS) in the 28 $\mathrm{GHz}(27.5-28.35 \mathrm{GHz}), 37 \mathrm{GHz}(37-38.6 \mathrm{GHz})$, and $39 \mathrm{GHz}(38.6-40 \mathrm{GHz})$ bands, and a new unlicensed band at 64-71 GHz. In Europe, the frequency band $3400-3800 \mathrm{MHz}$ is considered as the pioneering frequency band for $5 \mathrm{G}$ networks in Europe before the year 2020 given that it is already harmonised for mobile networks and satisfies the IMT-2020 channel bandwidth requirement for the lower 5G frequencies. On December 2016, the European Conference of Postal and Telecommunications (CEPT) recommended the 24.25-27.5 GHz as a pioneer band for $5 \mathrm{G}$ above $24 \mathrm{GHz}$ and encouraged the European partners to undertake the necessary spectrum harmonisation efforts and make the band available for national operators by the year 2020. On June 2016, Japanese Ministry of Internal Affairs and Communications (MIC) published their final report on $5 \mathrm{G}$ spectrum allocation and planned to use $5 \mathrm{G}$ networks operating in the 27.5-29.5 GHz band in Summer 2020 during the Olympic Games in Tokyo. Additionally, national policy makers and regulators are considering early 5G trials in the $3600-4100 \mathrm{MHz}, 4405-4895 \mathrm{MHz}$ and $27.5-28.28 \mathrm{GHz}$ bands. On January 2017, Korean Ministry of Science and ICT (MSIT) announced the K-ICT Spectrum Plan in order to use the $28 \mathrm{GHz}(26.5-29.5 \mathrm{GHz})$ band for 5G. On June 2017, Chinese Ministry of Industry and Information Technology (MIIT) is consulting wireless industry leaders on the mmWave spectrum planning in two principal candidate bands: the $24.75-27.5 \mathrm{GHz}$ and $37-42.5 \mathrm{GHz}$. At the time of writing this Thesis, no decision has been made on this aspect by the Chinese government.

With regard to the description of the mmWave spectrum use plans in each region, 3GPP actively studied the frequency bands in the region between 24 and $30 \mathrm{GHz}$ for NR in a WI which crystallised in the TR 38.815 document [15]. 


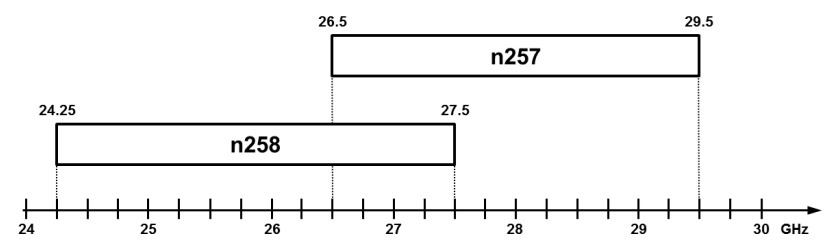

Figure 1.3: First standard mmWave bands for NR [15].

Finally, only two of the four original candidate bands in FR2 made it to the final specification document: standard bands n257 and n258. From lower to higher frequency, n258 ranges from $24.25 \mathrm{GHz}$ to $27.5 \mathrm{GHz}$ and $\mathrm{n} 257$ consider operation frequencies between $26.5 \mathrm{GHz}$ and $29.5 \mathrm{GHz}$. Both frequency bands are large, $3.25 \mathrm{GHz}$ and $3 \mathrm{GHz}$ bandwidths respectively. Figure 1.3. Note that the overlapping frequencies of the two frequency bands is $1 \mathrm{GHz}$ bandwidth.

\subsubsection{GPP Releases}

This subsection aims at describing the novelties brought by the $5 \mathrm{G}$ Phase 1 and Phase 2 to put the attention on the new services and applications.

\section{Release 15}

After initial delivery in late 2017 of NSA NR specifications, much effort focused in 2018 on completing the Release 15 specification documents in due time and on passing the first milestones imposed by the IMT-2020 time plan.

The first drop of the initial Release 15 specifications centred on NSA 5G network deployments integrated in the previous-generation LTE network architecture. The late versions of Release 15 standard documents cover SA 5G with a new core network. Release 15 also brings enhancements to LTE and, implicitly, its core network: the EPC. This crucial way-point enables vendors to progress rapidly with chip design and the early deployment of commercial $5 \mathrm{G}$ networks during 2019.

As the Release 15 work has matured and drawn close to completion, the focus shifted to the first stage of Release 16, also referred to as '5G Phase 2'. By the end of 2018, 83 studies relating to Release 16 were in progress for $5 \mathrm{G}$, covering diverse topics such as Vehicle-to-Everything (V2X) application layer services, 5G satellite access, Local Area Network (LAN) co-existence, terminal positioning and location, introduction of network automation processes and novel radio techniques, multimedia transmission, network slicing and Internet of Things (IoT). 
In addition, 3GPP groups are evaluating the applicability of 3GPP technology to satellites, airborne base stations, railway or maritime communications.

\section{Release 16}

Release 16 is a major Release for the $3 \mathrm{GPP}$ project since it will be a full $5 \mathrm{G}$ system compliant with the IMT-2020 guidelines. The completion of the Release 16 of NR is expected by March 2020.

Release 16 is the continuation of the services envisaged for Release 15 . Moreover, Release 16 centres on extending Mission Critical (MC) services to address a wider business sector than public security and civil defence services. The focus is on new commercial applications like taxi dispatching or railway traffic management. This would bring enhanced reliability to those MC services due to the wider adoption of the technology, which can reduce deployment costs due to economies of scale.

\subsubsection{Multi-antenna transmission in 3GPP}

\section{Evolution along LTE releases}

Multi-antenna schemes are an integral part of the LTE standard from the very beginning of the technology. Generally, a number of transmission layers $N_{L}$ are mapped to a number of antennas $N_{A}$ by a $N_{A} \times N_{L}$ precoder matrix, with the limitation on the number of layers, also referred to as rank in LTE nomenclature, cannot be larger than the number of antennas, that is, $N_{L}<N_{A}$.

The number of antenna elements and layers has increased along LTE releases. In Release 8, the number of maximum antennas is 4 . In downlink, the maximum number of antenna layers is 4 , but commercial base stations supported 2 layers at maximum. Up to four layers is possible in the downlink although commercial deployments typically use only two layers. Conversely, uplink transmissions only supports a single layer. LTE Release 8 offers a theoretical maximum data-rate of $150 \mathrm{Mbps}$ in downlink with a rank 2 transmission and $20 \mathrm{MHz}$ of bandwidth, and a maximum data rate of $75 \mathrm{Mbps}$ in uplink.

Release 10, also referred to as LTE-Advanced represented more than a significant update of the LTE technology, since it brought important novelties to the standards, also to multi-antenna transmissions [16]. The maximum number of supported layers and antenna ports in downlink augmented to 8. But more importantly, LTE Release 10 redefined the Reference Signal (RS) structure, and introduced separated RS for channel estimation for data and control planes. This redefinition was due the irruption of new transmission techniques in the Radio Access Technology (RAT) such a Coordinated Multi-Point (CoMP). 


\section{CHAPTER 1. INTRODUCTION}

Releases 13 and 14 focused on supporting very-large or massive antenna arrays, up to 64 antenna elements, and in Channel State Information (CSI) feedback enhancements. Among the enhancements, Releases 13 redefined the base station Transceiver Architecture (TXRU) [17], which separates the analog and digital precoding stages to better support beamforming. In addition, a beamformed Channel State Information Reference Signals (CSI-RS) was introduced that impacted the CSI channel training procedure and feedback, introducing the beam index report. The larger degrees of freedom obtained from the very large number of antennas can be used to create directional links to various users, i.e. to beamform. In this MU-MIMO, users are spatially multiplexed and each link conveys dedicated user data, which means that the same timefrequency resource can be shared among users. 3GPP recognises the spatial multiplexing advancements in LTE as Full Dimension MIMO (FD-MIMO).

LTE Release 15 extended the maximum number of antenna ports to 32 . The maximum number of receive antennas in downlink was increased to 8 . Therefore, up to 8 data streams could be transmitted to a single User Equipment (UE) with 8 antennas. 3D beamforing was introduced in this release, so beams can be configured not only in the horizontal plane but the vertical plane. On the other hand, Release 15 uplink supports the simultaneous transmission of 4 data streams from a user terminal with 4 antennas.

\section{Channel state information feedback}

The transmission rank, as well as the exact precoder matrix, can be selected by the network based on CSI measurements reported by the UE to the evolved Node B (eNodeB). This channel information feedback operation is known as closed-loop spatial multiplexing. This Thesis focuses on closed-loop reporting, but there are other strategies contemplated in the standards.

LTE allows spatial multiplexing in rank 1 channels. In this case, the precoder matrix is a $N_{A} \times 1$ matrix, that is, a precoder vector. This precoding vector can be used to beamform by selecting the precoding vector values or codeword out of a set of predefined precoding vectors or codebook. For this reason, this beamforming strategy is known as codebook beamforming.

In codebook beamforming transmissions, the UE gets the CSI from the transmitted Channel state information Reference Signal (CRS). Then, the user reports the eNodeB its perception of the channel state by sending a Precoding Matrix Indicator (PMI). Then the eNodeB applies the spatial domain precoding on the transmitted signal taking into account the PMI so that the transmitted signal matches with the channel experienced by the UE. Note that the PMI may be changed by the eNodeB according to the instantaneous state and then will be sent back to the UE. After precoding, the UE receives 


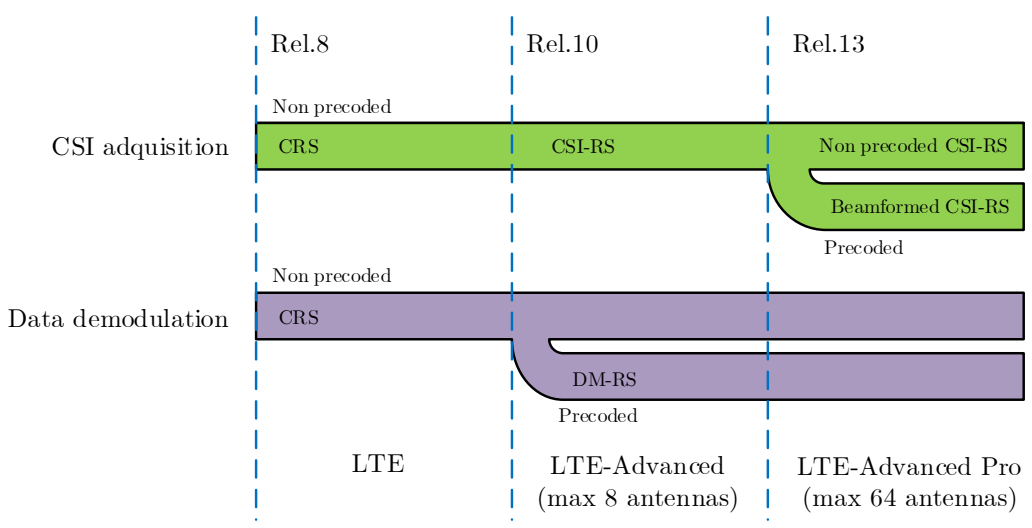

Figure 1.4: Evolution of RS in LTE.

the information from the eNodeB on what precoding matrix is used, which is utilised by the UE for demodulating the data.

Before Release 10, channel estimation is entirely performed by measuring CRS. LTE-Advanced introduced two new RS: CSI-RS and Demodulation Reference Signal (DM-RS) for CSI acquisition and data channel demodulation, respectively. Figure 1.4 illustrates the evolution of the RS use in LTE separated by data planes. The transmission frequency of the reference signals was altered. CRS transmission occurs every millisecond, whilst CSI-RS is transmitted with a periodicity of a multiple of $5 \mathrm{~ms}$. Similarly to CRS, the CSI-RS signals are transmitted to every user in the cell. On the contrary, DM-RS is UE-specific and individually addressed to the intended UE, so DM-RS signals are precoded or beamformed with the same weights applied to the data transmission channel. Since the DM-RS is present in the transmission grid under scheduling basis, this RS is not valid for CSI measurements in LTE.

Release 13 complemented CSI-RS with a beamformed version for CSI acquisition. Beamformed CSI-RS introduced a different channel training technique in LTE that uses multiple precoding weights to transmit the CSI-RS on different directions. The UE measures the received precoded CSI-RS and selects best received vector weights and reports its index to the eNodeB. This scheme is particularly beneficial over non-precoded CSI-RS for large number of antennas, since it reduces the uplink signalling overhead, the downlink pilot overhead, higher RS quality due to the beamforming gain [16]. 


\section{MIMO in NR}

The use of multi-antenna technology in NR is focused on two objectives. The first objective is to ensure sufficient coverage for NR deployment in over- $6 \mathrm{GHz}$ spectrum where propagation loss over wireless channels is significantly higher than that of sub- $6 \mathrm{GHz}$ spectrum. The second objective is to achieve spectral efficiency 3 times larger that in LTE systems.

Overcoming the large propagation loss is achieved in NR with beamformed transmission and reception, where the transmitter and receiver benefit from the antenna gain due to the large number of antenna elements. At a given time instance, data transmission to or from a base station is made using one of the multiple beams that can provide sufficient signal quality. Support for multi-beam operation in NR includes beam quality measurement, beam quality reporting, beam assignment, and recovery mechanism in case the assigned beam quality is not good enough. NR provides support for multi-beam operation at every stage of the radio operation: initial/random access, paging, data/control transmission/reception, and mobility handling.

\subsubsection{Channel models for simulations}

Standardised channel models have been widely used and have significantly contributed to the successful evolution of wireless network systems. This trend will undoubtedly continue in future developments. As a rule of thumb, standardised models must represent real channels with a certain level of precision, in addition to having an acceptable computational complexity; this makes them economically viable and advantageous compared to the normally expensive direct prototype of the technology to be developed. Generally, the degree of realism or accuracy of the model to be used depends ultimately on the scope of use of the model itself, so the computational cost can always be minimised. For example, a lower accuracy will be required in those cases in which you want to compare certain algorithms. In these cases, stochastic models have traditionally been used, which can be parameterised according to the use scenario: urban, rural, indoor, etc. On the other hand, a high degree of fidelity of the channel is necessary when planning a cellular network that must take into account both the antenna sites and their orientation or the morphology of the area under study. For the later case, professional planing software must be used. Such professional tools use channel models based on raytracing, which have a very high accuracy at the expense of a high computational load.

The most widely used standardised stochastic geometric models are the 3GPP Spatial Channel Model (SCM) [18], the 3GPP 3D [19] or the IMTAdvanced [20], which were formerly designed for frequencies below $6 \mathrm{GHz}$. 
These models have been improved to adapt to higher operation frequencies, up to $100 \mathrm{GHz}$. It is worth mentioning that the propagation conditions in the millimetre range are different due to the shorter wavelength of the signal. For example, diffraction tends to be smaller due to a reduced area of the Fressnel zone, scattering is higher due to the greater effective roughness of the materials and higher penetration losses [21]. The possible millimetre channel models will use common properties of low frequency systems such as multipath delay spread, angular dispersion or Doppler deviation, but with adequate values, and will also include new features such as high sensitivity to blocking due to buildings, people (even fingers) and strong differences between links with direct vision and no direct vision.

\subsection{State of the art analysis}

This section presents an analysis of the state of the art in the field of study of this Thesis based on an exhaustive literature review. This review is divided into five parts. The first one is the review of the MIMO antenna architectures. The second part discusses the channel estimation techniques. The third and last part deals with beam alignment techniques.

\subsubsection{Millimeter Wave communications}

The extremely-high data transmission rates and the service ubiquitousness expected in $5 \mathrm{G}$ require huge portions of bandwidth. The traditional operation frequency bands of wireless networks, which typically operate below $6 \mathrm{GHz}$, are highly saturated. Radio Access Network (RAN) in the unsaturated cmWave and mmWave bands have traditionally been discarded due to the higher wave propagation losses and blockage sensitivity in this frequency range, which usage seemed unfeasible in medium and long range communications. However, the large amount of free chunks of spectrum has attracted the interest of the scientific community in compensating the high propagation losses in the cmWave and mmWave bands. From the sake of notation simplicity and clarification of concepts, this Thesis will refer to cmWave as mmWave, even if the term is not used correctly. As the authors note in [22], it is well-known that free space propagation losses proportionally increase with the frequency, but the same formula applies to isotropic antennas with an effective aperture area of half the wavelength $(\lambda / 2)$. The higher the frequency, the shorter the wavelength and, therefore, the smaller the effective aperture area. On the other hand, an antenna with larger aperture has larger gain since it can capture more energy. 


\section{CHAPTER 1. INTRODUCTION}

Shorter wavelengths allows placing many antennas separated $\lambda / 2$ in the same effective aperture area. Having several antenna radiation elements makes the antenna being directional, meaning that the antenna can focus the energy around given directions, configuring lobes or beams. As a result, an additional antenna gain can be expected in the directions with maximum energy.

The combination of these two gains can overcome the free space propagation losses and additional losses from other agents which are not perceived in frequencies below $6 \mathrm{GHz}$ such as the foliage attenuation or rain attenuation. Authors in [22] experimentally demonstrate that the losses due to foliage can be very high, of up to $80 \mathrm{~dB}$, while the rain attenuation is negligible in communication link distances shorter than a few hundred meters. The authors in [23] foreseen the use of high gain directional antennas as an enabling technology of wireless communications in the mmWave band. In their work, authors also identify a series of elements to be taken into account in mmWave communications, such as 1) the shadowing probability behind the rapid fluctuations of the received wave signal level; and 2) coordination among users to reduce undesired interferences; and 3) the computational cost of the signal processing solution.

\section{Measurement campaigns above $6 \mathrm{GHz}$}

For the correct design of the future 5G system and its enabling technologies, it is necessary to correctly characterise the spatial and temporal propagation characteristics of the channels in the mmWave band in a wide variety of environments. The characterisation of mmWave channels gained momentum in the past five years with the explosion of $5 \mathrm{G}$ research activities. It is worth highlighting the significant contribution to this field of the research group led by professor Theodore S. Rappaport of the New York University (NYU). Some of their numerous experiments are public, like the outdoor measurement campaigns at the NYU campus in Downtown Manhattan at 28, 38 and $73 \mathrm{GHz}$ [23] or the repetition of the measurement campaign in the campus of the University of Austin in Texas [24]. The measurement campaigns basically consisted on placing a couple of directive horn antennas in different locations (with precise location information from Global Positioning System (GPS)), one antenna being the transmitter and the other antenna is the receiver, and determine the existence of direct vision between them or not. Both antennas were rotated in azimuth and elevation in order to obtain the departure and arrival angles of the different received power contributions, which is known as Packet Data Protocol (PDP), which have great relevance when obtaining the greater possible signal in conditions of no direct vision.

In both scenarios, Rappaport showed that in conditions of Line of Sight (LoS), the multipath component is practically irrelevant and the direct ray 
predominates, whereas in Non Line of Sight (NLoS) conditions the power is received from a few significant contributions, finding 3 maximum contributions in the outdoor Manhattan measurement campaigns. For these findings, mmWave channels are supposed to be low ranked, being the rank the number of successful rays. Channel statistics can be extracted from the PDP information such as the average delay spread Delay Spread (DS). In the measurement campaigns, the DS was about $200 \mathrm{~ns}$ in the Manhattan experiments, whereas the DS was $70 \mathrm{~ns}$ in Austin. In light of these results, it can be said that in verydense urban environments there are more reflections so the contributions are received with greater delay and attenuation. Rappaport also draw the attention on an important reflection: the construction materials in very dense cities introduce larger propagation losses at mmWave frequencies. Despite the larger attenuation due to reflections, all the measurements experiments in Manhattan reported received Signal to Noise Ratio (SNR) values of $10 \mathrm{~dB}$ at all distances below $200 \mathrm{~m}$ even in NLoS conditions due to the large antenna gain of the transmit and receive horn antennas, providing a joint gain of $50 \mathrm{~dB}$.

As for the probability of outage, all locations in Austin registered sufficient signal for distances shorter than 200 metres away from the base station. The measurement campaigns in Manhattan showed that at $28 \mathrm{GHz}$ the probability of a user to be out of coverage, i.e. in outage, is $14 \%$ for all receivers below 200 metres away from the base station. At $73 \mathrm{GHz}$, the outrage probability slightly increased to $17 \%$. These outages percentages are due to the higher probability of blocking the signal in dense urban scenarios, where $57 \%$ of the outage locations were in either in NLoS condition or the receiver was place further than 200 metres away from the transmitter. Finally, the authors also noted that in LoS conditions, the beam gain requirement can be relaxed.

In light of these results, the Rappaport group does not hesitate to claim that cellular systems operating in mmWave is feasible in ultra-dense urban environments, where the cell density is high and, therefore, the coverage area of the cells can be lowered and the LoS probability is larger. Communication is also possible in short-range NLoS conditions.

Rappaport's group went further in their measurement campaigns and repeated the study in a varied indoor environment for frequencies of $28 \mathrm{GHz}$ and $73 \mathrm{GHz}$ as detailed in [25]. The main novelty introduced was the study of the polarisation diversity. The main results drawn from the indoor experiments are a greater isolation between vertical and horizontal polarisation which led to a larger diversity. On the other hand, polarisation misalignment severely penalises (up to $32 \mathrm{~dB}$ of loss) if horn antennas are used as Maccartney showed in his evaluation. Antenna arrays with circular polarisation, or antenna panels with cross-polarisation should be used to minimise losses due to polarisation mismatches. 


\section{CHAPTER 1. INTRODUCTION}

Other initiatives worth noting in the characterisation of the mmWave channels are the Samsung initiative in Daejeon, South Korea, which conducted a statistical characterisation of the $28 \mathrm{GHz}$ band $[26,27]$ in a similar procedure to Rappaport's, or the characterisation undertaken by Salous in Durham University [28], who designed a 2x2 MIMO wideband sounder operating at $60 \mathrm{GHz}$, with a firm intention to extend the frequency to $90 \mathrm{GHz}$. The TU Ilmenau [29] has focused on characterising the $70 \mathrm{GHz}$ band in indoor environment.

In light of all the measurement campaigns detailed so far, it can be said that mmWave cellular communication systems are viable since useful signal levels were received. Measurement campaigns were undertaken in static conditions, with fixed transmitter and receiver. There is very little contribution in terms of the characterisation of the mmWave channel in mobility scenarios, and such characterisation is of great importance given the fact that one millimetre displacement at this large frequencies can completely change the perception of the channel. It would also be interesting to consider channel models with transmitter mobility to support Vehicle-to-Vehicle (V2V) type communications.

\section{Channel models above $6 \mathrm{GHz}$}

The partners of the METIS project were the first to review the existing channel models, identifying the model deficiencies that make them unsuitable for high frequencies. In their study [30], METIS consortium focused on the 3GPP 3D models [19], Winner / IMT-Advanced [20, 31, 32] and their extensions, COST 2100 [33] and Institute of Electrical and Electronic Engineers (IEEE) 802.11ad [34]. The main limitations that arose were the following:

- Inadequate bandwidth. As you go up in frequency the bandwidth is usually also higher, even greater than the maximum bandwidth allowed by the models.

- Two-dimensional models. 3GPP SCM and IMT-Advanced / WINNER $[20,31]$ are $2 \mathrm{D}$; that is, the diffraction and reflector phenomena occur on the same plane. This fact limits the use of MIMO techniques to the azimuth domain. Key elements and scenarios of $5 \mathrm{G}$ as $3 \mathrm{D}$ beamforming need the three coordinates of space. Therefore, it is necessary to use 3D models such as [19] or it would be necessary to introduce modifications to the SCM models that add the elevation component as proposed in [35], which provides similar values compared to the 3D slope of IMT-Advanced / WINNER [32].

- Low suitability for device-to-device (D2D) communications. The models only suppose movement in one of the two elements involved in the communication and does not contemplate that both ends are mobile, so that 
effects such as Doppler at both ends must be introduced. In addition, the authors emphasize that adapting the WINNER / IMT-Advanced models to D2D would be highly inefficient. These models estimate very well the correlation of the large-scale parameters based on a map of the precalculated area with a fixed base station. Having mobility in transmission and reception required to have a map for each location combination of the mobile nodes, increasing the dimensions of said map (from $2 \mathrm{D}$ to $4 \mathrm{D}$ or $6 \mathrm{D})$. Ignore the correlation of the meters for large scale, especially shadowing, will lead to erroneous predictions [36].

- Inconsistency of the small-scale parameters, in the sense that the spatial variety is not modelled realistically. The position of the diffraction elements or scatterers is determined stochastically, so that very close receivers can see different scatterers when they should be very similar. Especially affects the determination of the angles of departure and arrival, referred to in the literature as Angle of Departure (AoD) and Angle of Arrival (AoA) respectively, and whose determination is fundamental for a good design of beamforming solutions and beamtracking. In contrast, future models should be more realistic and with an improved spatial correlation.

- Lack of support for arrays of large antennas. The models require a much higher and more realistic resolution, in addition to supporting spherical and non-planar waves as in all two-dimensional stochastic models analysed. This last characteristic is of vital importance in order to validate multi-antenna systems.

In the report [37] the authors collect the measures initiatives and modelling of channels below $100 \mathrm{GHz}$ for $5 \mathrm{G}$, among which are included in this thesis proposal. The authors stress that the $3 \mathrm{D}$ model of the $3 \mathrm{GPP}$ must be the basis on which to build the future stochastic geometric models for the $5 \mathrm{G}$, and also detail the fulfilment of the necessary attributes to satisfy a $5 \mathrm{G}$ model. The $3 \mathrm{GPP}$ presented the first version of their 3D channel model for frequencies above $6 \mathrm{GHz}$ [5] in June 2016 and took the form of a Release 15 as a channel model from $0.5 \mathrm{GHz}$ to $100 \mathrm{GHz}$ in June 2018 [6].

Parallel to the development of the 3GPP models for very high frequencies, Professor Rappaport's group developed the first statistical channel model for high frequencies. The NYU channel model is based on their measurement campaigns with highly directional channel sounders, for which it was necessary to obtain the equivalent omni-directional propagation loss models by adding the received powers in each direction of aiming; that is, adding the areas below each of the PDPs for each angle, for each combination of elevation and azimuth for 


\section{CHAPTER 1. INTRODUCTION}

each pair of transmitter and receiver and eliminating the effect of the antenna gains, as detailed in [38]. Similar to 3GPP or IMT models, the NYU channel model is of the alpha-beta-gamma (ABG) style [39] where the coefficients are calculated in such a way that the Minimum Mean Square Error (MMSE) of the estimation is minimised for the operation frequency. But as already mentioned, communications in mmWave are directional, so Rappaport's group introduced in $[40,41]$ their preliminary 3GPP-3D-like model for Urban Micro-cell (UMi) and indoor environments, respectively. Both models are still alpha-beta-gamma (ABG) model type, with the difference of an additional propagation loss due to polarisation mismatch of the signal in the indoor model.

For a precise characterisation of the channel environment in a given scenario, professional channel models based on powerful ray tracing tools [42], completely established in the industry and used by professionals, and that are also helping to characterise the mmWave channels. These tools use multiple rays in three-dimensional representations of real scenarios to obtain very accurate propagation loss measurements. The only drawback of the ray-tracing tools is their high computational cost, which is why they are indicated for largescale channel models in which the propagation losses of each stage point are calculated only once. The partners of the METIS project have their so-called map-based model in which the propagation losses from each antenna to any outdoor position of their Madrid-grid scenario were calculated with a commercial ray-tracing tool [30]. The authors of [43] characterise two indoor scenarios in the mmWave band, from $26 \mathrm{GHz}$ to $30 \mathrm{GHz}$, in conditions of no direct vision to the transmitter, and corroborate the blockages that introduce people and objects in the signal and that the contributions with greater power they only come from a few addresses that can be grouped into a few clusters.

\subsubsection{Beamforming}

Signals transmitted in the mmWave bands suffer of propagation losses several orders of magnitude higher than those experienced by signals in the operation frequency which are typically used in cellular and other wireless communication systems. To overcome the excess in free-space propagation losses, systems operating in high frequencies must and can benefit from the additional gain provided by antenna arrays, which due to a shorter wavelength $\lambda$ results in higher density of antennas for the same effective area. The gain introduced by the array is directional. The energy is concentrated in certain directions where the signals coming from the different antenna elements sum constructively. These directions are influenced by the configuration of the array of antennas, this is, the number of antennas elements and the geometric shape of the array, and the spatial separation between adjacent antenna elements. By changing 
the amplitude and phase of the signal feeding the radiating elements, it is possible to steer the pointing direction of the main lobes. This is known in the literature as beamforming.

\section{Beamforming architectures}

In MIMO systems, the term of beamforming refers to the multi-antenna property of controlling the direction of a wave-front by appropriately adjust the magnitude and phase of the signals feeding each antenna element of the array. Considering the transmission case, the different copies of the signals transmitted from the antenna elements travels with different phases. In free-space propagation conditions, the signal level experienced in an arbitrary location is determined by the phase relationship among the copies. There are two extreme cases of signal phase interactions. On one hand, if signals arrive in opposite phases, they add destructively and, consequently, no signal is received. On the other hand, if the signals are in phase, the signals sum up constructively and the signal power level is significantly increased.

In LTE, the term MIMO usually refers to Single User MIMO (SU-MIMO). In SU-MIMO, both the Base Station (BS) and UE have multiple antenna ports and antennas, and multiple data streams are transmitted simultaneously to the UE, re-utilising the same time/frequency resources. Depending on the MIMO configuration, SU-MIMO doubles (2x2 MIMO), or quadruples (4x4 MIMO) the peak throughput experienced by the UE.

In MU-MIMO, the BS sends multiple data streams. Each flow is intended for a single UE. Like in SU-MIMO, data flows are multiplexed using the same time-frequency resources. As a result, the total cell capacity is increased. The BS has multiple antenna ports, at least as many as data streams or UEs, and each UE is mapped to a unique antenna port.

The transmission of multiple data streams (layers in 3GPP terminology) for different users is also known in the literature as precoding. Unlike beamforming, in which the phase shift of the signals emitted by the antenna elements is constant, in precoding the antennas are fed with different signals and also with different phases. The beam conformation in MIMO systems is done in baseband. This digital processing requires dedicated Radio Frequency (RF) chains, Analog-to-Digital Converter (ADC)s and power amplifiers per data stream. These components are not inexpensive, so the use of MIMO solutions with purely digital preprocessing is not feasible when the number of antennas is high. And so is the case of massive MIMO deployments. Note that precoding refers to the digital signal processing at the transmit side; the equivalent term for precoding at the receiver side is named combining. 
The number of RF chains, ADCs and power amplifiers, and their connection to each one of the antenna radiation elements in the array gives three different beamforming architectures:

- Precoding and MU-MIMO are also known as digital beamforming. This architecture requires separate RF and ADC chains for each individual antenna element in the array, enabling fully-digital signal processing. Multiple beams (one per user) can be formed individually for each UE and simultaneously to all the UEs served by the BS. The number of beam directions to form is potentially unlimited and it is only affected by the quantisation resolution of the ADCs used [44].

- Analog beamforming is the simplest beamforming architecture: it presents a single RF chain and it is connected to all the antenna elements of the array. Compared to the digital architecture, the analog architecture saves power, since only one power amplifier is used, and is more economical. Precoding is significantly less complex and computationally lightweight. The principal drawback of the analog beamforming architecture is that it can only form a single beam at a time. The number of beam directions to form is limited by the resolution of the phase shifters. Despite the aforementioned drawbacks, the reduced power consumption and complexity of analog beamforming architecture makes them an ideal candidate for early mmWave NR deployments.

- Hybrid beamforming is the compromise solution between digital and analog beamforming architectures. An hybrid architecture presents $N_{\mathrm{t}}$ antennas and $N_{\mathrm{RF}}^{\mathrm{t}} \mathrm{RF}$ chains, with the condition of $N_{\mathrm{RF}}^{\mathrm{t}}<N_{\mathrm{t}}$. The number of simultaneous data flows in an hybrid architecture is the number of RF chains $N_{\mathrm{RF}}^{\mathrm{t}}$. It is expected that $\mathrm{NR}$ deployments will evolve to some sort of hybrid transceiver configurations. One possible application in NR is performing beamforming in a two-step procedure: the analog beamforming would do beamforming with a wide beam, and then the digital precoder can fine tune and the beam to some extend.

\section{Massive MIMO}

The concept of massive MIMO was introduced by Thomas L. Marzetta [45] to refer to antenna array configurations with a very large number of antenna elements. It is commonly agreed that to properly refer to a massive MIMO system as massive, the number of antenna elements must be larger then than the number of potential users. In mmWave, a massive MIMO system typically consists of around a hundred radiant elements. 
A massive MIMO network may take advantage of the multiple degrees of freedom Degrees of Freedom (DoF) provided by the large number of antennas, more than users, for multiple purposes besides the beamforming gain, which details were provided in Subsection 1.2.2. The multitude of antennas may serve to transmit multiple data flows simultaneously using the same time-frequency resources, thus increasing the spectral efficiency of the base station. This is also known in the literature as spatial multiplexing. Furthermore, the multiple DoF can also be used to eliminate interference among users [46] and to reduce the complexity and precision of the hardware transceiver to reduce the economical cost. In a study on the effect of non-ideal hardware [47], the authors noted that, in effect, the hardware impairments reduce the potential capacity the massive MIMO system can achieve. However, the imbalance introduced by the user terminal is the most limiting factor.

When increasing the number of antenna elements, new interesting effects appear from a mathematical perspective. In his work, Marzetta noted that MIMO equations get important simplifications from the theory of random matrices. With very large number of antennas, the values of the MIMO channel matrices become deterministic functions instead of random variables. This characteristic allows introducing simplifications to the computational-intense channel matrix inversions. Furthermore, Marzetta proved that in MU-MIMO, when the number of antennas tend to infinite, linear coding in both base station and users is optimal even with users with a single antenna. In addition, the massive MIMO receiver can filter the thermal noise and fast fading effect just by averaging out the signals received in all its antenna elements, even in conditions of low SNR.

It is known that the performance of MIMO systems is penalised if favourable propagation conditions are not provided; that is, the channels perceived by the users must be sufficiently uncorrelated among them (they are mutually orthogonal) so the interference can be easily filtered out with linear coding. Most contributions in the MIMO literature assume rich scattering scenarios such as independent and identically distributed (iid) Rayleigh fading propagation environments, and preferably NLoS condition. However, mmWave communications are usually low ranked, with few significant contributions, given the elevated propagation losses and direct visibility conditions between user and BS according to Professor Rappaport's evaluation in [23]. Massive MIMO could overcome this axiom and provide good performance even in direct vision conditions due to the greater number of antennas in the transmitter, as suggested by the authors in [48]. In a latter work, Yang and Marzetta showed that in LoS situations, a massive MIMO system can achieve sum-rate values comparable to richer and more favourable idd Rayleigh channels with a simple power allocation strategy and selectively dropping certain users with large channel correlations [49]. 
Dropped users can be served in following subframes. In light of this result, the low ranked channel problem in a massive MIMO system can be tackled with an efficient scheduling policy.

The massive MIMO - mmWave binomial seems immediate. Fundamentally, the antenna spacing in an antenna array at mmWave is shorter compared to sub-6 GHz systems. As a result, more antenna elements can be fit in the same antenna form factor in mmWave. The extra antenna elements provides larger antenna gains, which compensates the high propagation losses at mmWave. However, the high number of antennas increases the complexity of the precoding stage at both the BS and user sides. If the number of antennas is very large, the computational cost of the fully-digital signal processing can be prohibitive with the current technology.

This is the fundamental motivation that impulsed the research of hybrid schemes for mmWave massive MIMO systems. Professor Robert W. Heath Jr. is one of the most prolific researchers in the field of hybrid precoding schemes for mmWave MIMO [50, 51]. Professor Heath noted the signal processing load is not negligible and represents a real challenge [44]. Given the dispersive nature of the mmWave channel, Professor Heath is inclined to use beam training algorithms based on predefined codebooks to find the best beams providing the largest beamformed channel gain. In his prior work with Alkhateeb [52], the authors propose an algorithm based on an iterative multilevel codebook in which, at each level, the aiming beam is sharpened until finding the contribution of the highest received signal power. In [53] the authors extend the hybrid precoding to a MU-MIMO system with limited feedback, that is, that the CSI information sent by the user is quantified, which affects the fine tuning of the beam. The algorithm, in its first phase, adjusts the analog beamforming by performing beam training in the BS and selects the beam that maximise the power received by each user. Later, in the second phase, the algorithm calculates the prcoding matrix in the base station to reduce the interference among users. Based on simulation results, the authors claim that hybrid architectures with this codebook-based beamforming offer similar performance in terms of cell capacity to the figures achieved by a purely digital beamforming architecture.

\section{Channel estimation}

The optimal operation point of a massive MIMO system is based on two assumptions:

- There is an exact knowledge of the channel response 
- User channels served by the same base station are completely uncorrelated.

Traditionally, wireless systems have transmitted pilot signals to estimate the response of the channel and adapt future transmissions to the channel response. This technique is based on the transmitter sending certain training sequences, distributed throughout the system bandwidth, which are known by the receiver. The relevance of acquiring information on the state of the channel or CSI is greater in massive MIMO than in traditional systems for two reasons. The first and fundamental reason is to concentrate the energy on the receiver. The second is that the energy efficiency that can be achieved with a massive MIMO solution is affected by the accuracy of the channel estimation.

Lets define the power efficiency as the power difference between a MIMO and Single Input Single Output (SISO) systems for the same sum-rate performance. Authors in [54] affirm that in a massive MIMO system with perfect knowledge of the channel state, the power efficiency is proportionally to the number of antennas of the base station, whereas if the CSI is not perfect, the maximum efficiency is proportional to the square root of the number of antennas of the base station. The acquisition of channel information can raise the signalling load, particularly if the number of antennas is high or the available bandwidth is large. The scientific community has put the attention in techniques to reduce the overhead due to CSI. The canonical massive MIMO system uses Time Division Duplexing (TDD) to take advantage of the supposed reciprocity of the channel response and thus prevent CSI reporting. With such strategy, the overhead depends on the number of terminals in spite of the number of antennas, which completely eliminates user scalability problems.

In [48] the authors ask themselves about the viability of massive MIMO systems operating in Frequency Division Duplexing (FDD) and authors found some important limitations. Note that in FDD channel reciprocity is no longer valid. As a result, users would need to report their CSI. Ideally, each user would report CSI feedback of each antenna of the base station. In an FDD massive MIMO, the signalling would significantly increase with the number of antennas, exhausting many time/frequency resources. Besides, the CSI values are only valid during the coherence time of the channel, which is inversely proportional to the carrier frequency and the user speed. In some very large antenna systems, it could not be possible to generate all the CSI reports within the same coherence time. Still, some research work was done to find alternative techniques to make massive MIMO systems feasible in FDD mode. Alternatives include using partial channel knowledge or limited feedback [55], or even blind methods in the sense that we do not use pilots to estimate the channel in FDD as in $[56]$. 


\section{CHAPTER 1. INTRODUCTION}

Pilot contamination has been addressed to be one of the most important limiting element of massive MIMO [46, 57]. Each user is assigned an orthogonal pilot sequence for their uplink transmissions, but the maximum number of orthogonal sequences is limited by the coherence interval divided by the delay spread. This is why this maximum number can become short for cellular systems. For instance, in a typical deployment such as the one presented by Marzetta in [58], only 200 codes are obtained for a coherence time of $1 \mathrm{~ms}$. Such a small number can be easily exhausted and, therefore, base stations will need to reuse those sequences. When users of contiguous cells use the same code, reception interference occurs and makes the uplink channel estimation incorrect. This is what is known in literature as pilot contamination. To model realistic conditions, we ought to add other sources of measurement contamination such as hardware non-idealities and imbalances or the non-reciprocity of actual transceivers. Nevertheless, the situation in uplink channel estimation is not pessimistic. Authors in [47] affirm that if the number of antennas is very large, the effect of pilot contamination can eliminated by simply averaging the received contributions. And according to Marzetta [58], pilot contamination phenomena in massive MIMO systems will neither be an issue. Authors in [50] explore alternative pilot-free channel estimation mechanisms for mmWave MIMO systems which use beamforming.

The authors of [59] give an exhaustive review of the pilot contamination problem in massive MIMO TDD systems and collect channel estimation solutions that mitigate the problem that appear in the literature recently. Marzetta and Appaiah propose in [60] to apply a temporal offset to the frames transmitted by each base station so that the pilots are not overlapped. This straightforward solution imposes the need to establish some coordination between the base stations involved. However, this solution might find scalability problems in high-dense heterogeneous networks where the cell density is very high and multiple BS may overlap their coverage areas.

Other authors suggest considering additional factors in the pilot sequence assignment in order to overcome the pilot contamination problem. For example, the spatial information of users can be exploited as suggested by the authors in [61], where they demonstrate that in an ideal assumption that useful and interfering signals reach the base station from different AoAs, inter-cell and intra-cell interferences can be completely eliminated if the number of antennas tends to infinity. However, the method requires obtaining second-order statistics of the massive MIMO uplink channels, which would be expensive to achieve. On the other hand, the works of $[62,63]$ completely eliminate the pilot contamination in precoding stage in TDD systems, using what they named Pilot Contamination Precoding (PCP), by including channel statistics such as 
the slow fading the users experience and reports to the BS. Sharing this type of channel information between cells is key to minimise inter-cell interferences.

\subsubsection{Beam tracking solutions}

One of the fundamental problems of directional communications is maintaining the link alignment. In the following subsections two possible approaches to solve the beam alignment problem are given. The first considers classical fingerprinting databases. The latter option considers Artificial Intelligence (AI) models, which is a modern research trend.

\section{Inverse Fingerprinting}

In a multipath environment, the signal power level at a given point largely depends on the topology of the scenario and the geometry of the blocking elements within. At a given point, the arriving wave contributions may combine constructively or destructively, resulting the latter in a coverage hole where no useful signal is received. Databases have been traditionally used by network operators to optimise certain network parameters such the list of neighbouring cells.

On a different note, databases may also assist in the user localisation or positioning. The channel conditions experienced by the UE can be seen as a sort of electromagnetic signature which is strongly correlated with the position of the user. The network operator can store in a database the collection of measurements and other network parameters reported by UEs. Each sample of the network parameter information is called a fingerprint. When a localisation procedure is needed, the network may instruct the UE to perform the necessary measurements and report them to the location server. The location server determines the UE location by comparing the received information with the fingerprints stored. The resolution of the fingerprint database determines the precision of the localisation estimation.

Lakmali's work in [64] introduced a localisation mechanisms for outdoor and indoor based on reporting Global System for Mobile Communication (GSM) signals. Lakmali achieved a localisation effectiveness of $33 \%$ in urban scenarios, and found an average localisation deviation error of $112 \mathrm{~m}$. In a latter work, Kupershtein et.al. [65] reported average localisation errors not larger than $1 \mathrm{~m}$ for indoor wireless networks with a fingerprint database considering the spatialtemporal evolution of the dominant contributions in multipath environments, demonstrating that accurate localisation estimations can be made.

The fingerprinting philosophy can also be applied to beams in mmWave communications. For example, Va et.al. introduced in their work in [66] an 


\section{CHAPTER 1. INTRODUCTION}

inverse fingerprinting approach: based on the user location, the geo-located database relates the reported position to a list of candidate beams to monitor for that specific location. Authors show that this method works well in LoS scenarios. But its performance in highly-dynamic scenarios with blockages with different time intervals in NLoS needs to be evaluated.

\section{Machine learning}

Without any question, Machine Learning (ML) is one of the most promising technologies. According to Simeone [67], ML can offer an efficient solution when cost and time constraints are the main concerns of the problem to solve, or when the problem appears to be too complex to be properly modelled in its full generality. ML generally provides a suboptimal solution, and most of the times, the interpretability of the solution is uneasy and only limited to a number of applications.

There is an increasing interest in using ML in different aspects of wireless network. Due to the wide diversity of service requirements, applications and hardware equipment complexities, $5 \mathrm{G}$ networks will be as complex as ever. This high complexity makes traditional network parameter adjustment inadequate. Authors in [68] suggest using novel self-aware, adaptive, and predictive optimisation algorithms in $5 \mathrm{G}$ networks. Network operators should use information from their network and subscribers to continuously monitor and optimise their networks. In the authors' opinion in $[68,69]$, this network intelligence can be obtained with the combination of Big Data analysis and ML.

The scientific community is interested in ML applications for all the network levels, including the Physical (PHY) layer. Authors in [68] suggest maintaining radio-electric maps reflecting the spatiotemporal evolution of the channel environment to make predictions of the signal fluctuations. This information can also be used to make suggestions on the optimised beamforming vectors to use at a given moment. In their survey, authors in [70] provide different research trends of ML applications in PHY, naming cognitive radio, energy harvesting, Massive MIMO, or coverage planning for ultra-dense networks.

The fundamental problem which is halting the development of ML applications for $5 \mathrm{G} \mathrm{PHY}$ is the lack of real measurements. Measurements are a fundamental part of the beam tracking problem, and without them, it is impossible to train an ML algorithm. Authors in [71] attempt to fill this gap and provide a small free-to-access database with measurements obtained from Remcom's InSite professional planning tool [72], which use a raytracer to accurately characterise the electromagnetic wave propagation conditions in real-world deployments. They use different snapshots (static scenario) to train the ML model. Measurement resolution is one square meter, which might be too large 
considering the mmWave wavelength size. To reduce the computational burden of the ray-tracing software used, obstacles like vehicles remain fixed during the scene; the receiver however is allowed to move during the scene. Ray tracing models are better than stochastic channel models because of the spatial consistency, which must be modelled in the latter models. Authors assume a good knowledge of the vehicular communication scenario used, including the number of lines, the type of vehicles - car, truck or bus - and the location of the vehicles. Authors evaluate a number of classification algorithms (the most complex deep neural network outperforms from the rest of learning algorithms) and a deep reinforcement learning approach.

Authors in [73] use a classification method approach to the beam pair selection in vehicular-type communication. Authors assume what they call "situational awareness," which consists of the system knowing the location of the surrounding vehicles and their type, which can be either a car or a truck. Such knowledge is obtained from other systems which can be available to the wireless network. This information is best known as side-band or out-of-band information. ML algorithms predict the best AoA directions, which match a specific beam in the beamforming codebook. As features, authors feed their ML networks with AoA and received power levels (given as quantised Channel Quality Indicator (CQI)) obtained from Remcom InSite ray tracing software. Results show that as long as a complete out-of-band information is provided, the overhead due to beam alignment can be practically eliminated without reducing the tracking ability nor the data rate experienced by the UE. However, the situational awareness seems an optimistic simplification of the problem: this information might not always be available nor be accurate. For this reason, authors in [74] extend the previous work and assume some practical issues that can occur in realistic environments such as GPS data inaccuracy, outdated locations given the reporting delays and missing features in the ML algorithm.

Authors in [75] use a type of Recurrent Neural Network (NN) (RNN) to predict the beam sweeping pattern according to the users spatial distribution. Their goal is to cover all users with the minimum number of beams, thus reducing the beam sweeping procedure timing. The spatial distribution of users is inferred from data in Call Detail Records (CDRs) of a real cellular network in Milan. Results show that the users spatial distribution and their approximate location (direction) can be accurately predicted based on CDRs data using the RNN model, which is then used to calculate the sweeping pattern in the angular domain during cell search. This certainly helps reducing the beam area search and reducing the initial connection delay.

An example of predicting the best beam without any out-band or side information is Alkatheeb's work in [76]. In their work, the authors train a neural network with past information of best past beams of a vehicle moving along a 


\section{CHAPTER 1. INTRODUCTION}

street in a V2X communication situation. The ML solution predicts whether or not the vehicle is about to experience blockage (the LoS link will not be available and connection should be hand-off to another base station with LoS). Therefore, the ML algorithm is a centralised solution and some degree of interactivity among base stations is required. In a similar system approach, the work in [77] use ML to leverage uplink channel training. In their systems, authors suggest using uplink training sequences or pilots in the sub-6 Ghz band using omnidirectional antennas. This is a two-step procedure: first, omnidirectional and beamformed measurements are done, taking most of the transmission time for training, and; second, only omnidirectional pilots are transmitted, freeing resources for data transmission. Authors noted the limited range of received SNR, which limits how far receivers must be from the transmitter, but the advantage is training overhead is practically non-existing once the ML network is trained according to authors. Four mmWave base stations, strategically located around the transmitting vehicle, receive the omnidirectional pilots and help constructing a spatial signature of the transmitter, which helps locating the transmitter. The location error is smaller than GPS accuracy. The envisaged deep learning solution use the spatial signatures to predict the beamforming weights.

Va, Heath et.al. in [78] introduced an online learning beam selection algorithm based on user positioning. The reinforcement learning beam selection algorithm has two stages. In the first step, the UE scans the angular space using a progressive phase-shift codebook. The beam sweep is done on a subset of promising beams or directions. In the second stage of the algorithm, the angles of the maximum gain directions of both base station and UE are fine tune to optimise the total beam gain. The envisaged reinforcement learning solution cannot be considered as fully online, given the fact that the database relating locations and candidate beams must be initialised offline by the method detailed in Va's previous work in [66], for the initialisation of first beam search procedure. The reward function feeding in this reinforcement learning approach uses binary values, relating to the selection of the optimum beam pairs (1) or not (0). The selection of a binary function limits the amount of valuable information feeding the neural network, but the selection of this criteria was necessary in order to reduce the complexity of the solution, facilitating the online training. In addition, the optimal reward function the authors considered in first place need measurements on all possible beams combinations. This is clearly unfeasible and authors rewarded on selecting the beam pairs with maximum gain out of a subset of promising beams. Authors claim that 30 promising beam pairs are needed in order to beam train, which seems a reasonable amount of beams for a massive MIMO deployment. Training with more beams will reduce the time resources devoted to data transmission, since an IEEE 802.11ad network 
is assumed. In the beam refinement procedure, authors assume an error area or bin size, ranging from 2 metres to 5 meters, which might seem over-sized for pedestrians.

Authors in [79] presented an AI-based AoA estimation for beamforming weight determination algorithm for multi-user MIMO. The beamforming weights decision is seen as a supervised learning problem, and weights are extracted out of a predefined Discrete Fourier Transform (DFT) codebook. Since the beamforming weights must cover with different beams users in different directions, authors need to widen the beams, hence reducing the beamforming gain. In order to reduce complexity, authors required hybrid beamforming architectures, which are a feasible solution for real mmWave network deployments. Different ML networks are evaluated and their learning is based on snapshots with fixed positions and conditions. This makes the current model not compatible with mobility environments, since every movement within a wavelength would need to re-train the ML network.

\section{Real measurements over $6 \mathrm{GHz}$}

The fundamental limitation to effectively evaluate any beam management solution, and for mmWave communication networks in general, is the limited amount of accessible realistic measurement data collections. Due to this limitation, most contributors to the beam management problem in the literature as Alkhateeb, Heath, or Va tend to rely on professional ray-tracing tools such as Remcon's InSite to obtain a propagation characterisation very close to reality. These professional tools have two important drawbacks:

- The computational cost to run this software is expensive.

- Normally, this tools takes static scenarios or snapshots where the blocking objects and the transmitter and receivers are fixed. Moving any element on the static scenario implies re-calculating all the measurement points.

- A commercial license to use this kind of professional software is required, and for some researchers the cost can be prohibitive.

To fill this gap, some altruistic initiatives have arisen and provide freeaccess data sets of mmWave campaigns. Table 1.2 lists some of the open-access initiatives for mmWave or ML.

The database in [71] contains more than 40, 000 measurement of 10 vehicles, acting as users of the mmWave network, on a 200 meters long section of a three line road with other vehicles, seen as obstacles. Measurements are arranged in 116 episodes, and each episode contain 50 scenes or static snapshots of 
Table 1.2: Summary of mmWave free-access collections of propagation data

\begin{tabular}{|c|c|c|c|c|}
\hline Initiative & Summary & Source & Frequency & Scenario \\
\hline $\begin{array}{l}\text { 5GMData } \\
{[71]}\end{array}$ & $\begin{array}{l}\text { More than } 40,000 \\
\text { measurement } \\
\text { points of a three- } \\
\text { lane road section } \\
\text { for a ML model for } \\
\text { V2I communica- } \\
\text { tions. }\end{array}$ & $\begin{array}{l}\text { Ray- } \\
\text { tracing }\end{array}$ & $60 \mathrm{GHz}$ & Outdoor \\
\hline $\begin{array}{l}\text { Deep } \\
\text { MIMO } \\
{[80]}\end{array}$ & $\begin{array}{l}\text { More than } \\
1,000,000 \text { mea- } \\
\text { surement points in } \\
\text { a V2X scenario in } \\
\text { the intersection of } \\
\text { two streets }\end{array}$ & $\begin{array}{l}\text { Ray trac- } \\
\text { ing }\end{array}$ & $60 \mathrm{GHz}$ & Outdoor \\
\hline $\begin{array}{l}5 \mathrm{G} \\
\text { mmWave } \\
\text { Channel } \\
\text { Model } \\
\text { Alliance } \\
{[81]}\end{array}$ & $\begin{array}{l}\text { NIST and collab- } \\
\text { orating partners } \\
\text { maintain a col- } \\
\text { lection of real } \\
\text { measurements. }\end{array}$ & $\begin{array}{l}\text { Real } \\
\text { measure- } \\
\text { ments } \\
\text { from } \\
\text { NYU } \\
\text { Wireless, } \\
\text { Qual- } \\
\text { comm, } \\
\text { NIST, } \\
\text { and } \\
\text { others }\end{array}$ & $\begin{array}{l}\text { From } \\
2.4 \mathrm{GHz} \text { to } \\
300 \mathrm{GHz}\end{array}$ & $\begin{array}{l}\text { Outdoor } \\
\text { and in- } \\
\text { door, } \\
\text { multiple } \\
\text { scenarios }\end{array}$ \\
\hline $\begin{array}{l}\text { DeepSig } \\
{[82]}\end{array}$ & $\begin{array}{l}\text { A dataset of } \\
\text { recorded RF sig- } \\
\text { nals with different } \\
\text { digital and analog } \\
\text { modulations for } \\
\text { ML. }\end{array}$ & Synthetic & $\mathrm{NA}$ & NA \\
\hline $\begin{array}{l}\text { Signal } \\
\text { Metadata } \\
\text { Format } \\
\text { (SigMF) } \\
{[83]}\end{array}$ & $\begin{array}{l}\text { A GNU Radio data } \\
\text { format to share } \\
\text { signal } \\
\text { ments. } \\
\text { some Provides } \\
\text { waveforms } \\
\text { ples. }\end{array}$ & Synthetic & NA & NA \\
\hline
\end{tabular}


the channel conditions and location of the 10 receivers and other vehicles. This dataset was created with the sole intention of demonstrating that a ML approach can be given to the problem of predicting the optimal combination of transmit and receive beams. For each measurement point, the database contains information on the number of rays arriving at that location, the AoA and AoD in both azimuth and elevation planes, and the delay and attenuation of each ray. Even if the number of measurement points is large, the time resolution of the data collection is $100 \mathrm{~ms}$, which might be very large for some complex mmWave system models with more exigent time resolution demands.

The dataset in [80] is the same data source used in Alkhateeb's ML works [77] and [76]. The scenario selected is a two street intersection with about 4,000, 000 measurement points. However, the open-access data contains information of one single channel realisation and with no obstacles in the scenario.

The 5G mmWave Channel Model Alliance [81] is the most extensive collection of real measurement campaigns data in an open-access form. The Alliance, sponsored by the National Institute of Standards and Technology (NIST), was created "to facilitate improved data and knowledge sharing amongst leading communications engineers interested in producing more accurate and predictive channel models and measurements required to support the commercialisation of next generation wireless networks (5G and beyond)". Up to date, the database contains waveform measurements from 50 contributors such as Qualcomm, NYU Wireless, Georgia Institute of Technology, Communications Research Centre Canada, or NIST. Some examples of the measurements included in the dataset are given below:

- Indoor path-loss models at 28, 29, 60, 73, 140, and $300 \mathrm{GHz}$.

- Outdoor path-loss models at 38 and $73 \mathrm{GHz}$.

- Data-center-type communications at $60 \mathrm{GHz}$.

- Semi-Open and closed office measurements at 2.4, 3.5, 5.8, 26, 38, and 61 $\mathrm{GHz}$.

- Lobby hallway and basement propagation measurements at $83.5 \mathrm{GHz}$.

- Shopping Mall signal propagation measurements at 2.9, 29, and $60 \mathrm{GHz}$.

\subsection{Problem formulation and Thesis scope}

This Thesis focuses on investigating the link adaptation problem of mmWave communications for NR networks under user mobility. The mmWave band 


\section{CHAPTER 1. INTRODUCTION}

presents the disadvantages of experiencing large propagation losses and being very sensitive to blockage phenomena. It is widely agreed that MIMO solutions are called to solve the propagation problem in mmWave frequencies. MIMO ability to beamform the transmission and reception of signals adds a significant beam gain, which is vital to the success of the mmWave communication link. Many research activities have focused on channel estimation in mmWave links finding the precoding matrices that optimise a given performance metric, e.g. the cell capacity or user throughput. However, those same studies assume random channel realisations of the MIMO channel, and there is a lack of performance evaluation in realistic propagation scenarios.

Characterising scenarios in a realistic manner is necessary for the correct evaluation of any beam management strategy since the different rays arriving to the receiver depend on the geometry of the scenario. The geometry counts for the number of rays arriving at destination and also for the number of times a given ray bounces on different reflectors. This Thesis aims at providing a realistic MIMO channel model for mmWave communications supporting 3D beamforming and antenna arrays with very-large (massive) number of radiation elements.

Many contributions in the literature to the channel sounding problem rely on the transmission of non-standard pilots. Pilot transmission side effect is allocating time/frequency resources for such transmission. The overhead due to pilot transmission scales up with the number of the transmit antennas. In massive MIMO system, when the number of antennas is very large, pilot transmissions might exhaust the available resources, reducing the network capacity. One objective of this Thesis is keeping the pilot overhead to a minimum. To that aim, channel information will be obtained from the standardised Synchronization Signal (SS), which are transmitted periodically and, therefore, no additional overhead might be required. The Thesis tries to identify scenarios where this strategy may actually work.

Previous work on mmWave communication networks evaluated the performance of their solutions based on performance figures at the PHY level. There is little work in assessing the impact of beam misalignment in end-to-end network simulators. Another objective of this Thesis is to provide a standardised mmWave beam management framework within an end-to-end $5 \mathrm{G}$ network simulation tool.

In order to support user mobility, the channel information associated to users must be periodically updated. However, full channel knowledge from standardised SS in NR might be delayed in time. In beamformed communications, full channel information knowledge implies performing and reporting measurements of each combination of transmit and receive beam. The challenge is to update all possible beam combinations in a reasonably short period of 
time. Instead of performing an exhaustive beam search throughout the whole beam space, it would be desirable to get fast updates of promising beams. Then, the objective is finding those promising beams in an efficient and accurate way. Such mechanisms may also relax the dependency of beam tracking on measuring the periodically-transmitted SS, and the network could eventually extend the SS transmission period in order to allocate less resources to pilots and devote more resources for data transmission and, therefore, increasing the network capacity.

To conclude the Thesis outline, the main objectives of the Thesis are summarised as follows:

- Propose and evaluate mmWave beam tracking strategies which are in alignment with 3GPP Release 15 of NR. Beam tracking strategies will make use of the collection of beam measurements of standard downlink RS and The envisage strategies must make an efficient use of the time/frequency resources available in the system, keeping signalling overhead to a minimum.

- Study alternative beam tracking strategies which make use of user location or other information sources.

- Provide a realistic MIMO channel model adjusted to massive MIMO based on real-world propagation conditions.

- Provide a whole simulation framework for beam management using standardised protocols and signals.

\subsection{Thesis outline}

This Thesis is organised into 7 chapters. The present chapter is the first one and serves as an introduction to the mmWave beam tracking problem in NR. The rest of the Thesis is structured as follows:

- Chapter 2 presents the main features of 3GPP NR Release 15 which are the foundations of the management and tracking strategies of the mmWave beams. More precisely, the Chapter centres the attention on the PHY and higher layer aspects.

- Chapter 3 introduces the system model for the evaluation of the beam tracking strategies in the mmWave bands. Two channel models are used to recreate the multipath MIMO channel: 3GPP channel model for larger frequencies and a ray-tracing tool built in Unity, an open-source video 
game engine. This ray-tracing channel model was specifically created for this Thesis.

- Chapter 4 establishes the simulation environment, which is the main evaluation method used in this Thesis. The selected simulation tool is the well-known end-to-end network simulator ns-3. This chapter presents the mmWave module for ns-3 and deepens on the modifications introduced in this Thesis work to the PHY and high layers to be Release 15 compliant. A section of this chapter is devoted to detailing a visualisation platform created in Unity to visualise network-related information in the context of a real city.

- Chapter 5 defines the beam tracking strategies devised in this Thesis. The proposed beam tracking strategies are based on the collection and use of measurement reports. These reports are generated by wellconfigured UEs, which are mandated to measure certain downlink RS in certain time/frequency resources. The beam tracking strategies make use of the standardised RS used for synchronisation and initial access: the SS/Physical Broadcast Channel (PBCH)-Block (SSB). The SSB-based tracking may be complemented by periodic CSI-RS reports.

- Chapter 6 implements beam tracking strategies which use additional information sources or tools to suggest the NR system which beams to use in both the transmit and receive side. The proposed solutions include, first, a database which relates the user position with the optimal pair of beams; and, secondly, a machine learning model to estimate the following pair of beams to use.

- Chapter 7 draws the main conclusions of the research work conducted in this Thesis and gives some insights on the possible research work activities for the future.

\subsection{Publications}

The work developed during this Thesis made possible the publication of the following journals, conference papers, project reports with special relevance and a book chapter. A set of publications resulting from work closely related with the Thesis is also provided. 


\section{Journals}

[J1] C. Herranz, E. González and J. F. Monserrat, "New Radio Beam Management in Release 15," Accepted for publication in IEEE Communication Standards Magazine on July 16th 2019.

[J2] D. Martín-Sacristán, C. Herranz, J. F. Monserrat, A. Szczygiel, N. P. Kuruvatti, D. García-Roger, D. Prado, M. Boldi, J. Belschner, and H. D. Schotten, "5G Visualization: The METIS-II Project Approach," Mobile Information Systems, 2018.

\section{Conferences}

[C1] C. Herranz, M. Zhang, M. Mezzavilla, D. Martín-Sacristán, S. Rangan, and J. F. Monserrat, "A 3GPP NR Compliant Beam Management Framework to Simulate End-to-End mmWave Networks," 21st ACM International Conference on Modeling, Analysis and Simulation of Wireless and Mobile Systems (MSWIM), Montreal, Canada, November 2018, pp. 119-125.

[C2] C. Herranz, D. Martín-Sacristán, J. F. Monserrat, N. Cardona, S. Inca, "On the Use of Serious Game Engineering for 5G System Performance Evaluation" 2016 European Conference on Networks and Communications (EuCNC), Athens, Greece, June 2016, pp. 1-5.

\section{Technical reports with special relevance}

[R1] METIS-II, "Final 5G Visualization," Deliverable D7.3, Jun. 2017

[R2] METIS-II, "Preliminary 5G Visualization," Deliverable D7.2, Dec. 2016

\section{Related work}

[W1] D. Martín-Sacristán, C. Herranz and J. F. Monserrat, "Traffic safety in the METIS-II 5G connected cars use case: Technology enablers and baseline evaluation," 2017 European Conference on Networks and Communications (EuCNC), Oulu, Finland, June 2017, pp. 1-5.

[W2] J. Cabrejas-Peñuelas, D. García-Roger, I. Alepuz-Benaches, C. Herranz, S. Gimenez-Colás, "Tecnologías SON" in 3GPP LTE-Advanced y su evolución hacia la 5G móvil, Marcombo, 2017. 



\section{Chapter 2}

\section{GPP NR Release 15}

This Thesis is oriented towards the study of beam tracking strategies within the ambit of the New Radio (NR) specifications defined by Third Generation Partnership Project (3GPP). In order to perform any assessment, it is necessary to have a deep knowledge of the system protocol under study before defining an evaluation methodology. This chapter of the Thesis deals with the first issue.

This chapter deals with the principal aspects of the 3GPP specification documents released for Fifth Generation (5G), also referred to as NR, which are directly related to the beam tracking problem in the Millimeter Wave (mmWave) band. This chapter is focused on Standalone (SA) NR networks operating in the mmWave band or in Frequency Range (FR) 2 (FR2) in 3GPP terminology.

With this aim, this chapter has been divided into the following sections:

- Section 2.1 presents the structure of the new Physical (PHY) layer.

- Section 2.2 deals with the higher layers mechanisms that enable the beam management framework.

- Section 2.3 draws the main conclusions of the present chapter.

\subsection{Physical layer aspects}

This subsection summarises the principal novelties in the PHY layer introduced by $3 \mathrm{GPP}$ which are directly connected to multi-antenna techniques in FR2. 


\subsubsection{Flexible radio frame structure}

In order to accommodate the new 5G services such as Ultra-Reliable LowLatency Communications (URLLC) and the use of high carrier frequencies, the frame structure needs a certain degree of flexibility. Some of the assumptions made in Long Term Evolution (LTE) are still valid in the new standard. As an example, NR continues using Orthogonal Frequency Division Multiplexing (OFDM). However, the separation in frequency among consecutive OFDM subcarriers or Sub-Carrier Spacing (SCS), is flexible and not fixed to a single value as in the LTE case. Taking the SCS in LTE as reference, $\Delta f_{\text {base }}=15 \mathrm{kHz}$, the possible SCS values in NR are multiples of the reference SCS [84]:

$$
\Delta f=\Delta f_{\text {base }} \cdot 2^{\mu}
$$

where the scaling factor $\mu=[0,1,2,3,4]$

Each combination of SCS and cyclic prefix Cyclic Prefix (CP) length is referred to as numerology. The motivation behind having different numerologies is to address different types of services, and CP is mainly motivated to protect subcarriers from the Doppler effect. The choice of a given numerology depends on various factors: type of deployment, service requirements, possible hardware impairments, user mobility, performance and implementation complexity [85].

The complete list of numerologies that can be applicable in NR are listed in Table 2.1. It is worth noting that different numerologies apply to different FR. Smaller SCS values are supported for FR 1 (FR1) $(15,30$ and $60 \mathrm{kHz})$, whereas the largest SCS values only apply to $\operatorname{FR2~(60,120~and~240)~[86].~According~}$ to the specification, numerologies can accommodate data and control physical channels, with two exceptions: $60 \mathrm{kHz}$ SCS can only be used for data channels; and $240 \mathrm{kHz}$ SCS can only transport control data. In FR2, SCS values of $60 \mathrm{kHz}$ and $120 \mathrm{kHz}$ are to be expected for data transmission, whereas SCS values of $120 \mathrm{kHz}$ or $240 \mathrm{kHz}$ are meant for transmitting $\mathrm{NR}$ control information, mainly cell synchronisation signals. The SCS does not necessarily need to be announced to users in the control information. This is not a problem because the User Equipment (UE) may derive the SCS from the carrier frequency.

Moreover, Downlink (DL) and Uplink (UL) transmissions are multiplexed in time within frames with a fixed duration of $10 \mathrm{~ms}$, being each frame further divided into 10 subframes with a fixed duration of $1 \mathrm{~ms}$. This transmission arrangement in time is exactly the same used in LTE. The difference in NR is that the SCS value determines the number of OFDM symbols in a subframe. The specifications define a slot as a group of 14 consecutive OFDM symbols, except for the case of using Extended CP where the number of symbols in a slot is 12 . As a result, the number of slots per subframe is not fixed, as it occurred in LTE, and its value entirely depends on the SCS configuration. Figure 2.1 
Table 2.1: The different numerologies can be applied to different CP, frequency bands and data planes.

\begin{tabular}{|c|c|c|c|c|}
\hline $\boldsymbol{\mu}$ & $\mathbf{S C S}(\mathbf{k H z})$ & Cyclic prefix & FR & Physical channel \\
\hline \hline 0 & 15 & Normal & FR1 & Control/Data \\
\hline 1 & 30 & Normal & FR1 & Control/Data \\
\hline 2 & 60 & Normal/Extended & FR1/FR2 & Data \\
\hline 3 & 120 & Normal & FR2 & Control/Data \\
\hline 4 & 240 & Normal & FR2 & Control \\
\hline
\end{tabular}

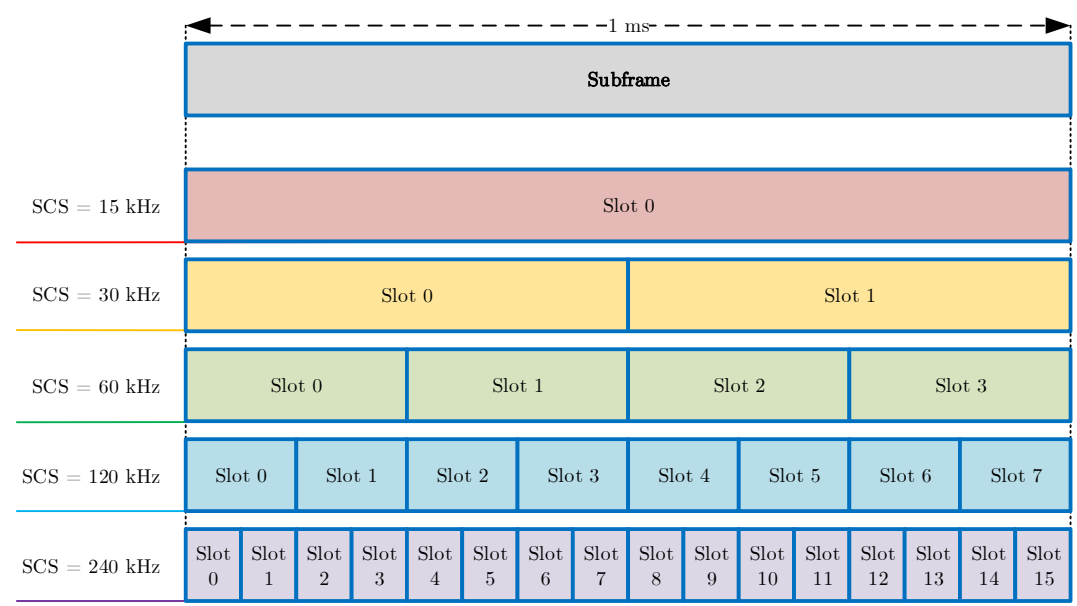

Figure 2.1: The number of slots that fit in one subframe $(1 \mathrm{~ms})$ varies with the SCS.

illustrates the number of slots in a subframe for the supported SCS values and normal CP. Note that the number of slots can be directly obtained as the SCS value divided by the reference SCS value of $15 \mathrm{kHz}$.

Another interesting aspect of the new radio frame in NR is the possibility of grouping 2 consecutive OFDM symbols to form a mini-slot in the NR nomenclature. Mini-slots are of particular interest for low-latency URLLC services, since scheduling decisions can be made at a mini-slot granularity.

Another novelty introduced in NR is that the OFDM symbols within a slot can be mapped to be DL or UL. This is different to LTE Time Division 


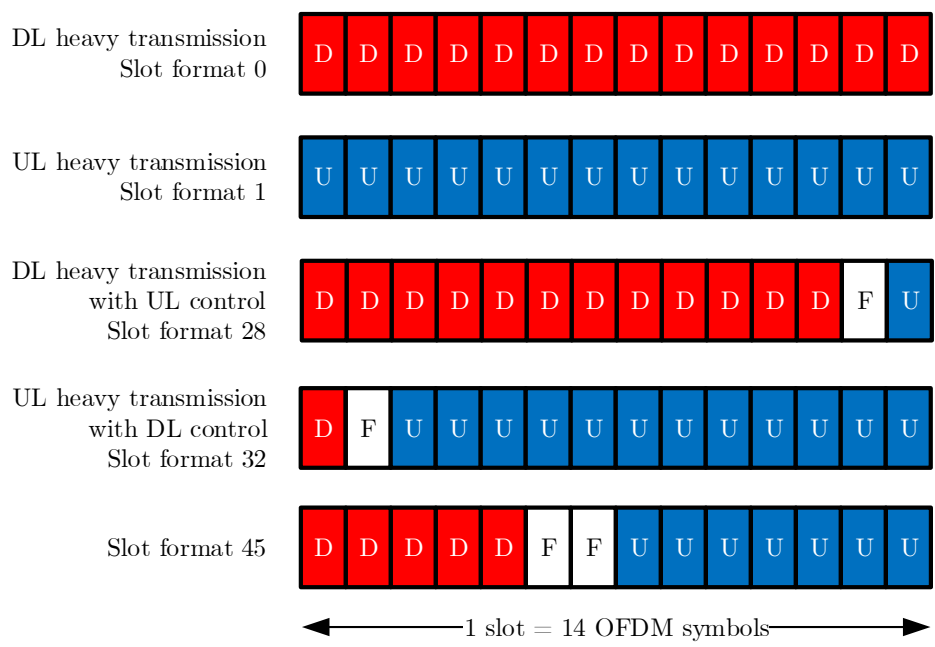

Figure 2.2: Some examples of the slot formats for NR.

Duplexing (TDD) systems, in which a subframe and all of its symbols can only be configured either for downlink or uplink transmission. NR defines several slot formats [86], which determine how the symbols within a slot will be used, that is, which symbols will be marked for DL and which symbols will be mark for UL. The number of slot formats is potentially large. In practice, 3GPP specifies 61 slots formats in Release 15; slot formats 62 to 128 are reserved. Most of the slot formats have in common that DL symbols are mapped at the beginning of the slot and UL symbols are located at the end of the slot. Slot formats contemplate the use of special cases called as flexible. Flexible symbols can be used for downlink or uplink transmissions indistinctly; the decision on how to use the flexible symbols is up to the scheduler. Some examples of slot formats are given in Figure 2.2.

In NR, a single slot can be divided into multiple segments of consecutive symbols marked as DL, UL or flexible.

In frequency, an OFDM subcarrier is the minimum allocation entity or Resource Element (RE). More precisely, a RE is made of one OFDM subcarrier and one OFDM symbol. A Resource Block (RB) contains 12 subcarriers and 1 slot or 14 OFDM symbols, which makes 168 REs. In NR Release 15, one carrier has a maximum number of 3300 subcarriers, which represents 275 RBs. 
Table 2.2: Summary of the radio frame parameters that depend on the SCS. The number of slots and OFDM symbols are given on a per subframe and per frame basis. The symbol duration influences the minimum and maximum numbers of RBs and bandwidth for a single component carrier.

\begin{tabular}{|c|c|c|c|c|c|}
\hline SCS (kHz) & $\mathbf{1 5}$ & $\mathbf{3 0}$ & $\mathbf{6 0}$ & $\mathbf{1 2 0}$ & $\mathbf{2 4 0}$ \\
\hline \hline Number of slots per subframe & 1 & 2 & 4 & 8 & 16 \\
\hline Number of slots per frame & 10 & 20 & 40 & 80 & 160 \\
\hline OFDM symbol length $(\mu \mathrm{s})$ & 66.67 & 33.33 & 16.67 & 8.33 & 4.17 \\
\hline Number of symbols per subframe & 14 & 28 & 56 & 112 & 224 \\
\hline Number of symbols per frame & 140 & 280 & 560 & 1120 & 2240 \\
\hline Minimum RB & 24 & 24 & 24 & 24 & 24 \\
\hline Maximum RB & 275 & 275 & 275 & 275 & 138 \\
\hline Minimum bandwidth $(\mathrm{MHz})$ & 4.32 & 8.64 & 17.28 & 34.56 & 69.12 \\
\hline Maximum bandwidth $(\mathrm{MHz})$ & 49.5 & 99 & 198 & 396 & 397.44 \\
\hline
\end{tabular}

Another novelty in NR is the possibility of defining Bandwidth Parts (BWPs), which is a subset of contiguous RBs within a carrier [84]. The maximum number of BWPs per transmission direction (downlink or uplink) is four and, at a given time, a UE can only have one active BWP per link. For instance, the network can dynamically change from a narrow BWP to a wider BWP in case the UE data rate needs grow.

Table 2.2 summarises the numerologies supported by 3GPP for NR, including the number of slots and OFDM symbols for each SCS per frame and subframe. The table also contains the minimum and maximum number RB for each SCS, which results in different bandwidths.

\subsubsection{Synchronisation and initial access in NR}

In LTE, base stations or evolved Node B (eNodeB) transmit Synchronization Signal (SS) so a UE in their vicinity can detect the presence of the eNodeB.

The synchronisation procedure is a keystone procedure in every wireless network. Mobile stations perform channel measurements in order to recognise a known signal which helps them get the fundamental information needed to get connected to the base station. In LTE this information is conveyed in Primary Synchronization Signal (PSS) and Secondary Synchronization Signal (SSS).

In NR, PSS and SSS are packed together with Physical Broadcast Channel $(\mathrm{PBCH})$ in a single block, which is known as SS/PBCH-Block (SSB). The structure in time and frequency of an SSB is depicted in Figure 2.3. The block spreads in time over 4 OFDM symbols. In frequency, the SSB occupies 240 


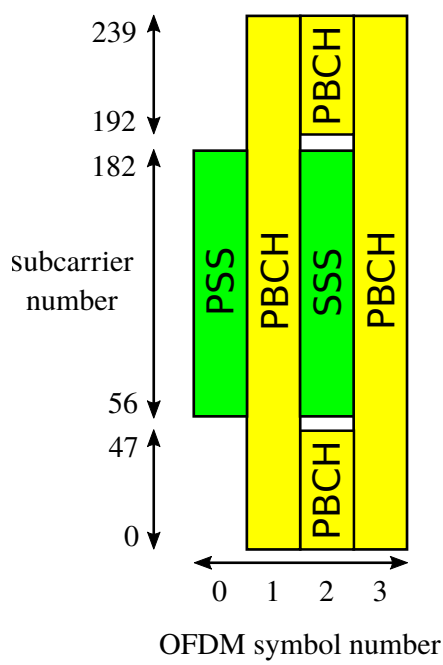

Figure 2.3: Illustration of the periodic SSB directional transmission over time.

contiguous subcarriers or $20 \mathrm{RB}$. Unlike in LTE, the location in frequency of the SSB is not fixed. In LTE,PBCH, PSS and SSS are located at the centre of the carrier bandwidth. The position of SSB in frequency domain is identified by two higher layer parameters naming offsetToPointA and ssb-SubcarrierOffset.

SSB transmissions occur within a half frame, i.e. $5 \mathrm{~ms}$, which is referred to as SS burst set in NR terminology. The possible locations in time of the SSBs depend on the SCS. All locations are referenced to OFDM symbol 0, which corresponds to the first symbol of the first slot of the SS burst set [86]. Table 2.3 lists the different patterns or cases of the starting OFDM symbols $(\Delta S)$ of the SSB in the SS burst. As it can be seen, the SSB positions depend on the SCS and frequency. Cases A, B and $\mathrm{C}$ can be used for sub- $6 \mathrm{GHz}$ frequencies, and cases D and $\mathrm{E}$ for mmWave band. Additionally, Table 2.3 details the maximum number of SSB transmission occasions $(L)$ within an SS burst, which depends on the operation frequency: $L=4$ for FR1 frequencies below $3 \mathrm{GHz}, L=8$ for FR1 frequencies over $3 \mathrm{GHz}$ and below $6 \mathrm{GHz}$, and $L=64$ for frequencies in FR2.

The SS burst structure is periodically repeated. The NR standard contemplates periodicity values are 5, 10, 20, 40, 80 and $160 \mathrm{~ms}$ [86]. This period repetition, which is larger or equal than the SS burst length, gives the sense of a burst SSB transmission. For initial access, by default the SSB transmission window is repeated every $20 \mathrm{~ms}$ [86]. Nevertheless, the network may announce 
Table 2.3: Starting OFDM symbol indices $(\Delta \mathrm{S})$ of SSB within the SS burst set for the possible SCS vales and maximum number of SSB transmissions $L$.

\begin{tabular}{|c|c|c|c|c|c|}
\hline \multirow{2}{*}{ Case } & \multirow{2}{*}{$\begin{array}{c}\text { SCS } \\
(\mathrm{kHz})\end{array}$} & \multirow{2}{*}{$\Delta \mathrm{S}$} & \multicolumn{2}{|c|}{ FR1 } & \multirow{2}{*}{$\begin{array}{c}\text { FR2 } \\
\text { f }>6 \mathrm{GHz}\end{array}$} \\
\hline & & & f $<3 \mathbf{G H z}$ & $\mathrm{f}>\mathbf{3} \mathrm{GHz}$ & \\
\hline \multirow{2}{*}{ A } & \multirow{2}{*}{15} & $\multirow{2}{*}{2,8}+14 n$ & $\mathrm{n}=\{0,1\}$ & $\mathrm{n}=\{0,1,2,3\}$ & \multirow{2}{*}{ NA } \\
\hline & & & $L=4$ & $L=8$ & \\
\hline \multirow{2}{*}{ B } & \multirow{2}{*}{30} & \multirow{2}{*}{$\begin{array}{r}\{4,8,16,20\} \\
+28 \mathrm{n}\end{array}$} & $\mathrm{n}=\{0\}$ & $\mathrm{n}=\{0,1\}$ & \multirow{2}{*}{ NA } \\
\hline & & & $L=4$ & $L=8$ & \\
\hline \multirow{2}{*}{$\mathrm{C}$} & \multirow{2}{*}{30} & $\multirow{2}{*}{2,8}+14 n$ & $\mathrm{n}=\{0,1\}$ & $\mathrm{n}=\{0,1,2,3\}$ & \multirow{2}{*}{ NA } \\
\hline & & & $L=4$ & $L=8$ & \\
\hline \multirow[t]{2}{*}{$\mathrm{D}$} & \multirow[t]{2}{*}{120} & \multirow[t]{2}{*}{$\begin{array}{r}\{4,8,16,20\} \\
+28 \mathrm{n}\end{array}$} & \multirow[t]{2}{*}{ NA } & \multirow[t]{2}{*}{ NA } & $\begin{array}{r}\mathrm{n}=\{0,1,2,3,5,6, \\
7,8,10,11,12, \\
13,15,16,17,18\}\end{array}$ \\
\hline & & & & & $L=64$ \\
\hline \multirow[t]{2}{*}{$\mathrm{E}$} & \multirow[t]{2}{*}{240} & \multirow{2}{*}{$\begin{array}{r}\{8,12,16, \\
20,32,36, \\
40,44\}+56 n\end{array}$} & \multirow[t]{2}{*}{ NA } & \multirow[t]{2}{*}{ NA } & $\begin{array}{r}\mathrm{n}=\{0,1,2,3, \\
5,6,7,8\}\end{array}$ \\
\hline & & & & & $L=64$ \\
\hline
\end{tabular}

a different periodicity in the system information. More precisely, the network broadcasts in ssb-PeriodicityServingCell and ssb-PositionsInBurst elements of the System Information Block (SIB) 1 (SIB1), the SS burst set period and the SSB time index for the cell [87]. Additionally, the network can provision UEs with a SSB-based Measurement Timing Configuration (SMTC) value, which imposes a restriction on the SSB measurement duration and periodicity. This limitation on the subset of SSB to measure helps reduce the UE power consumption and save battery.

In FR2, the maximum number of SSB transmissions $(L)$ within the SS burst set $(5 \mathrm{~ms})$ is 64 , although the number of transmitted SSBs can be lower.

In NR network deployments, SSB cases may not be explicitly indicated when broadcasting the system information. In such case, the UE can deduce which case is used by detecting the central carrier frequency and the SCS. PBCH conveys an SSB time index, which helps identifying the current SSB within the SS burst set. The frequency location of the SSB, however, is configurable by upper layers and the SS do not need to be allocated at the centre of the frequency band as happens in LTE. The ssb-SubcarrierOffset field in the Master Information Block (Master Information Block (MIB)) announces the frequency offset in number of subcarriers between the start of the SSB and the beginning of the resource grid [87]. 
SSB transmission must be beamformed in FR2. This requires the NR base station (gNB) to alternate different beams pointing at different directions in order to transmit the SSB over the whole coverage area. This is known as beam sweeping. To identify beams in downlink, or transmit beams, each direction can be associated to a SSB time index.

\subsubsection{Channel State Information in NR}

NR can indistinctly operate in TDD or Frequency Division Duplexing (FDD). But mainly TDD. TDD can assume channel reciprocity, which eliminates the need of estimating downlink and uplink separately. The standard provides all the elements needed to perform channel sounding and obtain Channel State Information (CSI).

As mentioned in Section 1.1.3, Channel State Information Reference Signals (CSI-RS) were introduced in the Release 10 of LTE, which was the first Release of LTE Advanced (LTE-Advanced) [88]. CSI-RS were introduced to support $3 \mathrm{D}$ beamforming and to perform a more efficient channel sounding. Originally, CSI-RS supported up to 8 antenna ports, but this number has been extended in NR and supports up to 32 antenna ports in Release 15. A CSI-RS resource associated to a single antenna port occupies one RE.

Unlike LTE, the CSI-RS mapping in NR is not based on a defined pattern, but rather on a set of combinations and conditions that must be met. CSI-RS resources are spread on every RE in the active BWP and use the same numerology. However, CSI-RS cannot be allocated in those REs conveying an SSB or Demodulation Reference Signal (DM-RS) associated to the Physical Downlink Shared Channel (PDSCH). The CSI-RS resource configuration always refers to the active BWP. Nevertheless, the CSI-RS can be configured to cover just a fraction of the BWP. In such case, the network must instruct the UE the the starting RB index and the number of RB mapped with CSI-RS resources.

In the context of NR Release 15, the number of REs used for CSI-RS and their time-frequency locations depend on three parameters [84]:

- The number of CSI-RS ports, which can be 1, 2, 4, 8, 16, 24 or 32 .

- The CSI-RS resource density, which defines the number of RBs used for CSI-RS resource within the BWP. The density can take three possible values: $0.5,1$ or 3 . A density configuration of 1 means that CSI-RS will be transmitted in every RB within the BWP or the configured fraction of bandwidth. If the network provides a density value of 0.5 , CSI-RS will be transmitted in half of the RBs that compose the BWP, in odd or even RBs. The standard mandates that 4,8 or 12 antenna port cannot 
support CSI-RS density equal to 0.5 . The density value of 3 is only used for oscillator time and frequency tracking.

- Port multiplexing allows sharing the CSI-RS resources among a set of CSI-RS ports. Such resource sharing is possible with the use of orthogonal sequences in transmission, which cancels any possible interference within the shared resources. This technique is known as Code Division Multiplexing (CDM). The advantage of using CDM is that it balances the transmit power of the CSI-RS signal along its frequency resources. Depending on the code bit length determined by the upper layers, a number of 2, 4 or 8 ports can be multiplexed in the same CDM group. For larger number of port multiplexing, CDM can be combined with time Time Division Multiplexing (TDM) or frequency Frequency Division Multiplexing (FDM) multiplexing, or both options, and have all the 32 CSI-RS ports multiplexed.

The exact time/frequency resources reserved for a specific CSI-RS resource within an RE results from a process involving a pseudo-random sequence multiplied by weighting sequence in both time domain and frequency domain. The exact procedure is detailed in [84].

CSI-RS resources have three different time allocation strategies, naming periodic, aperiodic or semi-persistent reporting. For periodic and semi-persistent CSI-RS configurations, the UE assumes that the CSI-RS signals are transmitted with a periodicity of $T_{\mathrm{CSI}-\mathrm{RS}}$ slots and with an offset value of $T_{\text {offset }}$ slots, both defined by the network. This CSI-RS reporting is determined by upper layers in the reportSlotConfig parameter. For SCS of $60 \mathrm{kHz}$, the possible periodicities that can be configured are 40, 80, 160 or 320 slots, whereas the possible periodicities are doubled for the SCS value of $120 \mathrm{kHz}$. Regardless of the numerology used, the periodicities in time that can be configured are $10 \mathrm{~ms}$, $20 \mathrm{~ms}, 40 \mathrm{~ms}$ or $80 \mathrm{~ms}$. For periodic or semi-persistent CSI-RS strategies, only one CSI-RS resource set is given. In semi-persistent reporting, the transmission of CSI-RS can be dynamically activated or deactivated by the network by sending a Medium Access Control (MAC) Control Element (MAC CE) [89]. When the network activates the allocation of CSI-RS, the UE assumes the reporting periodicity from the moment it received the activation indication message until the allocation is explicitly deactivated.

A UE may report different CSI parameters [90], based on the network configuration, which most of them were already introduced in LTE: Channel Quality Indicator (CQI), Precoding Matrix Indicator (PMI), Layer Indication (LI) (Layer Indication), Rank Indicator (RI), or the newly introduced Layer 1 Reference Signal Received Power (RSRP) (L1-RSRP) for link adaptation measurements. In case of measuring beamformed signals, the UE sends a CSI-RS 
Resource Indicator (CSI-RS Resource Indicator (CRI)) with the CSI-RS report. CSI information is feedback through the Physical Uplink Control Channel (PUCCH) or the Physical Uplink Shared Channel (PUSCH).

\subsubsection{Downlink signals and overhead}

The transmission of Reference Signals (RSs) occupies a number of time/frequency resources which cannot be used for user data transmission. Therefore, it is important to evaluate the impact of the signalling overhead in the number of available resources and achievable data rate. This Thesis is interested in evaluating the contribution of the downlink RS to the signalling overhead, and no uplink RS will be considered.

In the first place, the contribution of the SSB to the signalling overhead is determined. As illustrated in Figure 2.3, in the frequency domain an SSB occupies 240 subcarriers, equivalent to $20 \mathrm{RBs}$, while in time it spans 4 OFDM symbols. During an SS burst period with duration $T_{\text {SSburst }}$ multiple SSBs may be transmitted. Let's use $L$ for the number of SSBs during an SS burst.

The product of time/frequency resources devoted to SSB transmissions, $R_{\mathrm{T}}^{\mathrm{SSB}}$, during a time interval with duration $T$, and considering a large $T$, tends to be equal to:

$$
R_{T}^{\mathrm{SSB}}=240 \times \Delta_{f} \times 4 \times T_{\text {symb }} \times L \times \frac{T}{T_{\text {SSburst }}}
$$

where $\Delta_{f}$ is the subcarrier bandwidth and $T_{\text {symb }}$ is the duration of each OFDM symbol including the cyclic prefix.

The valid values for $T_{\text {symb }}$ can be approximated by $T_{\text {symb }}=71.35 / 2^{n} \mu \mathrm{s}$ where $n=\log _{2}\left(\Delta_{f} / 15000\right)$. Therefore, the previous expression can be simplified to:

$$
R_{T}^{\mathrm{SSB}}=1027.44 \times L \times \frac{T}{T_{\text {SSburst }}}
$$

The total product of time/frequency resources available in a system during the same period of time, depends on the system bandwidth, BW:

$$
R_{T}^{\text {Total }}=\mathrm{BW} \times T .
$$

Then, the percentage of the total time/frequency resources devoted to the transmission of SSB is:

$$
\text { Overhead }_{\mathrm{SSB}}=\frac{R_{T}^{\mathrm{SSB}}}{R_{T}^{\text {Total }}} \times 100=\frac{1027.44 \times L}{\mathrm{BW} \times T_{\mathrm{SSburst}}} \times 100 .
$$

Note that the valid range of values for $L$ depends on the SCS configuration for control data as it can be seen in Table 2.3. Specifically for SCS up to $30 \mathrm{kHz}$, 
the maximum $L$ is 8 , while for higher bandwidths the maximum $L$ is 64 .Also the valid range of BW depends on the SCS configuration (see Table 2.2).

Table 2.4 shows the maximum and minimum signalling overhead obtained for different control data SCS values when the maximum $L$ is used for each SCS value. Lower overheads could be obtained if the number of SS bursts per burst period was lower. To obtain the minimum overhead shown in the table, the maximum system bandwidth for data transmission of $396 \mathrm{MHz}$ and the maximum SS burst periodicity of $160 \mathrm{~ms}$ have been considered. The maximum overhead values correspond to the minimum system bandwidth for data transmission of $4.32 \mathrm{MHz}$ and the minimum SS burst periodicity of $5 \mathrm{~ms}$.

Table 2.4: SSB overhead for different control data SCS.

\begin{tabular}{|c|c|c|}
\hline SCS (kHz) & 15 \& 30 & 120 \& 240 \\
\hline \hline Maximum overhead (\%) & 38.05 & 100 \\
\hline Minimum overhead (\%) & 0.01 & 0.10 \\
\hline
\end{tabular}

This Thesis assumes a common numerology for user and control data. Specifically it is used an SCS of $120 \mathrm{kHz}$ and the maximum system bandwidth for that SCS, which means a $\mathrm{BW}_{\mathrm{RB}}$ of $275 \mathrm{RBs}$. It also assumes that 64 SSB transmission occasions are used within an SS burst. In such case, the percentage of resources occupied by SSB is summarised in Table 2.5.

Table 2.5: Percentage of the overhead due to SSB transmission over the whole system bandwidth in this Thesis (one component carrier).

\begin{tabular}{|r|r|}
\hline SS burst period (ms) & SSB overhead (\%) \\
\hline \hline 5 & 3.32 \\
\hline 10 & 1.66 \\
\hline 20 & 0.83 \\
\hline 40 & 0.42 \\
\hline 80 & 0.21 \\
\hline 160 & 0.10 \\
\hline
\end{tabular}

The overhead due to CSI-RS signalling is calculated similarly. The CSI-RS are transmitted periodically with a period equal to $T_{C S I-R S}$. In one slot within this period, a configurable number of CSI-RSs related to different antenna ports can be accommodated. This number should lie within the set $1,2,4,8,16,24,32$. In frequency, for each CSI-RS, one RE (1 subcarrier during 
1 symbol), is reserved in every $\rho \mathrm{RB}$, where $\rho$ is the density parameter defined in Section 2.1.3. Note than $\rho$ can only take 1 or $1 / 2$ as value.

The product of time/frequency resources devoted to CSI-RS transmissions, $R_{\mathrm{T}}^{\mathrm{CSI}-\mathrm{RS}}$, during a time interval with duration $T$, and considering a large $T$, tends to be equal to:

$$
R_{T}^{\mathrm{CSI}-\mathrm{RS}}=\mathrm{BW}_{\mathrm{RB}} \times \rho \times \Delta_{f} \times T_{s y m b} \times B \times \frac{T}{T_{\mathrm{CSI}-\mathrm{RS}}}
$$

where $B W_{R B}$ is the system bandwidth measured in RBs and $B$ is the number of different CSI-RS transmitted.

The product of total time/frequency resources, $R_{T}^{\text {Total }}$, during the time interval $T$ is:

$$
R_{T}^{\text {Total }}=\mathrm{BW}_{\mathrm{RB}} \times 12 \times \Delta_{f} \times T .
$$

Then, the percentage of the total time/frequency resources devoted to the transmission of SSB is:

$$
\text { Overhead }_{\mathrm{CSI}-\mathrm{RS}}=\frac{R_{T}^{\mathrm{CSI}-\mathrm{RS}}}{R_{T}^{\text {Total }}} \times 100=\frac{T_{\text {symb }}}{12 \times T_{\mathrm{CSI}-\mathrm{RS}}} \times \rho \times B \times 100
$$

The overhead due to CSI-RS for the different CSI-RS periods and a SCS of $120 \mathrm{kHz}$ is listed in Table 2.6 .

Table 2.6: Percentage of the CSI-RS overhead with respect to the total number of resources available for the CSI-RS periods with an SCS of $120 \mathrm{kHz}$.

\begin{tabular}{|r|r|}
\hline CSI-RS period (ms) & CSI-RS overhead (\%) \\
\hline \hline 10 & $7.4 \cdot 10^{-3} \rho \cdot B$ \\
\hline 20 & $3.7 \cdot 10^{-3} \rho \cdot B$ \\
\hline 40 & $1.85 \cdot 10^{-3} \rho \cdot B$ \\
\hline 80 & $0.93 \cdot 10^{-3} \rho \cdot B$ \\
\hline
\end{tabular}

The contribution of CSI-RS to the signalling overhead is largely determined by the number of CSI-RS resources $B$. The density parameter $\rho$ can only be 1 or $1 / 2$ and can contribute to reduce the overhead. As it can be seen in Table 2.6, the base CSI-RS overhead with unitary $\rho$ and $B$ values is 3 orders of magnitude below the number of resources allocated for SSB transmission with the longest SS burst period of $160 \mathrm{~ms}$. The low CSI-RS overhead allows allocating large number of CSI-RS resources to measure the same number of combinations of transmit and receive beams without exceeding the overhead due to SSBs. It can be concluded that the contribution to the dowlink signalling 
overhead of CSI-RS resources is marginal for low number of $B$, and practically all of the overhead associated to the transmission of downlink RS is due to SSB transmissions.

\subsection{Higher layer aspects}

This Section discusses the upper-layers procedures and aspects that make an important part of the beam tracking framework.

\subsubsection{Beam management}

3GPP introduced beam management as a part of the new multi-antenna transmission technology in the study of the PHY requirements to support 5G [91]. 3GPP defines beam management as the collection of L1 and L2 procedures to acquire and maintain a set of Transmit Receive Point (TRP), i.e. one of the possible multi-antenna panels belonging to a gNB, and UE beams. Beam management is composed of 4 stages:, which are illustrated in Figure 2.4:

- Beam determination is the ability of the TRP or the UEs to select of its own beams.

- Beam measurement is the process to quantise the characteristics of the received beamformed signal.

- Beam reporting is the ability of the UE to report the beam information. This information is obtained from having performed a beam measurement procedure.

- Beam sweeping is the operation of covering a spatial area with transmit or received beams during a specific time interval and in a specific manner.

The Technical Report (TR) 38.802 also details the beam correspondence between TRP and UE. On one hand, the TRP can determine the set of downlink beams to use based on UE uplink beam measurements. In the same way, the TRP can also determine the set of uplink beams to use based on TRP uplink beam measurements. On the other hand, the UE can determine the set of uplink beams to use based on TRP downlink beam measurements. But, the TRP notifies the UE the set of downlink beams to use based on TRP uplink beam measurements. Beam correspondence relies on efficient beam measurement and beam reporting mechanisms. Note that an important simplification can be assumed in situations where downlink and uplink channel reciprocity is 

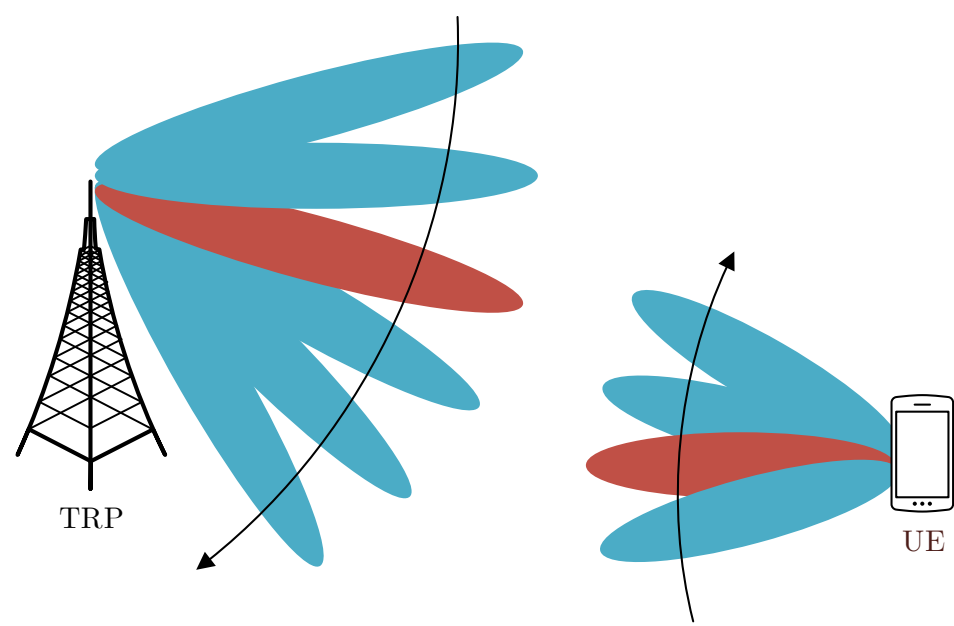

(a) Beam sweeping.

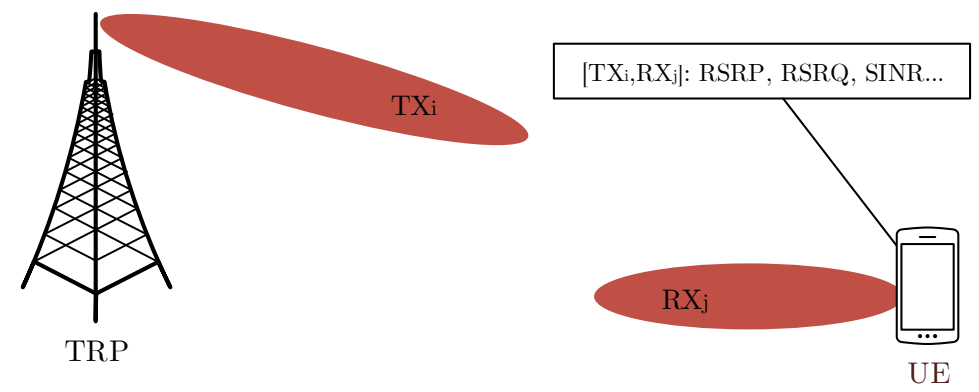

(b) Beam measurement. 


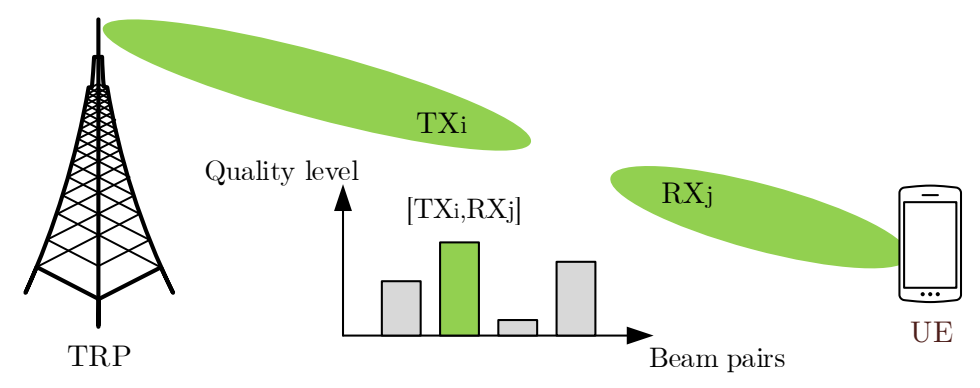

(c) Beam determination.

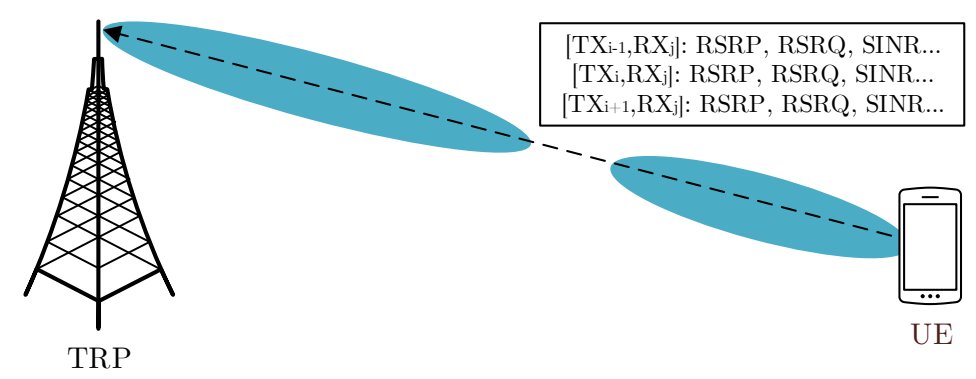

(d) Beam reporting.

Figure 2.4: The four beam management procedures studied by 3GPP during the PHY-study phase. 
guaranteed, which can be the case of TDD systems. In this case, uplink and downlink beams are the same.

Beam management can be seen as a continuous process which starts when a UE triggers the initial access procedure and continues as long as the UE is in the CONNECTED state. 3GPP TR 38.802 splits the process in three separated processes which are tightly coupled, which are $\mathrm{P}-1, \mathrm{P}-2$ and $\mathrm{P}-3$ processes.

$\mathrm{P}-1$ objective is enabling the UE to perform beam measurements on different TRP downlink beams and select the set of most promising TRP downlink beams. To this aim, the TRP performs beam sweeping and transmit the reference signals the UE has to measure on different TRP beams. In this stage, the UE also beam sweeps with its downlink beams.

P-2 is the procedure in which the UE measures different downlink TRP beams in case of changing the TRP downlink beam discovered in P-1. In P-2, the downlink UE beam is fixed. P-2 also performs beam sweeping on the TRP side, so it can be seen as a reduced case of P-1 with a smaller set of TRP downlink beams. P-2 can be used for beam refinement of the downlink TRP beam.

P-3 is the complementary procedure to P-2 on the UE side. The UE beams sweeps and measures a TRP beam, which is fixed, with different UE downlink beams.

\subsubsection{Beam measurement and reporting}

NR CSI framework is not a trivial procedure and involves Radio Resource Control (RRC) and PHY. The network instruct a UE in RRC_CONNECTED to perform and report measurements. The measurement configuration is provided by means of dedicated messages RRCReconfiguration or RRCResume. The network can configure measurements on NR or legacy networks, but in this description of the CSI framework the focus is on NR measurements on mmWave.

The network may configure the UE to report the following measurement information based on SSB or CSI-RS. Each signal has its advantages and disadvantages. On one hand, SSBs are used for initial access and the gNB always transmits this signal. Monitoring SSBs for CSI does not result in increased overhead. However, SSB spans along $20 \mathrm{RBs}$ and, therefore, rarely cover the whole system bandwidth. CSI-RS resource allocation is flexible and can be configured to cover any RE in the whole bandwidth. In addition, CSI-RS can be allocated more frequently if needed. The disadvantage of CSI-RS is the additional overhead.

The UE can filter the CSI measurement reports, so it can notify results per individual SSB or CSI-RS resource, or group SSB or CSI-RS measurements in 
a cell report. In addition, the UE can provide the resource identifier, that is, the SSB index or the CSI-RS resource identifier. This last feature is of extreme interest for beam management since it allows reporting the beam associated to the reported index, and SSB and CSI-RS resources are mapped to beams in a 1-to-1 basis.

The CSI framework includes five measurement configuration parameters:

1. First of all, a Measurement Object (MO) indicates the list of objects on which the target UE shall perform measurements. More precisely, a MO indicates the RB location and SCS of the RS to be measured. For a given $\mathrm{MO}$, the network may configure a list of cell specific offsets, a list of 'whitelisted' cells and a list of 'blacklisted' cells. The UE may perform measurements of cells which cell ids are contained the white list. On the contrary, the UE will not perform any measurement of cells which cell id is in the black list of cells. The network configures as many MO as serving cells. The measObjectId of the MO which corresponds to each serving cell is indicated by servingCellMO within the serving cell configuration.

2. Each MO has a list of associated reporting configurations. Each reporting configuration contains the following information:

- The measurement report criterion that triggers the UE to send a measurement report. This criterion can be a time periodicity condition or a triggering event.

- The type of RS the UE considers for beam and cell measurement results. The RS can be either SSB or CSI-RS.

- The reporting format determines the information reported by the UE. The format specifies the measurement quantities per cell and per beam that the UE includes in the measurement report (e.g. RSRP) and other associated information such as the maximum number of cells and the maximum number beams per cell to report.

3. A measurement identity links one MO with one reporting configuration. By configuring multiple measurement identities, it is possible to link more than one MOs to the same reporting configuration, as well as to link more than one reporting configuration to the same MO. The measurement identity is also included in the measurement report that triggered the reporting, serving as a reference to the network.

4. The quantity configuration defines the measurement filtering configuration used for all event evaluation and related reporting, and for periodical reporting of that measurement. For NR measurements, the network 
may configure up to 2 quantity configurations with a reference in the NR measurement object to the configuration that is to be used. In each configuration, different filter coefficients can be configured for different measurement quantities, for different RS types, and for measurements per cell and per beam.

5. A measurement gap is a period of time when the data transmission and reception of the addressed UE is deactivated. During the measurement gap period, as it name suggests, the UE perform measurements on other Radio Access Technologys (RATs).

An NR device can be configured to carry out different measurements, in most cases with corresponding reporting to the network. In general, such a configuration of a measurement and corresponding reporting are done by means of a report configuration, in the $3 \mathrm{GPP}$ specifications referred to as a CSIReportConfig.

Each resource configuration describes/indicates:

- The specific quantity or set of quantities to be reported;

- The downlink resource(s) on which measurements should be carried out in order to derive the quantity or quantities to be reported;

- How the actual reporting is to be carried out, for example, when the reporting is to be done and what uplink physical channel to use for the reporting.

A report configuration indicates a quantity or set of quantities that the device is supposed to report. The report could, for example, include different combinations of CQI, RI, and PMI, jointly referred to as CSI. For beam management purposes, the report quantity can be L1-RSRP, which is similar to RSRP used for in higher layers but without performing the (layer-3) filtering.

In addition to describing what quantity to report, a report configuration also describes the set of downlink signals or, more generally, the set of downlink resources on which measurements should be carried out in order to derive the quantity or quantities to be reported. This is done by associating the report configuration with one or several resource sets

A resource configuration is associated with at least one NZP-CSI-RSResourceSet to be used for measuring channel characteristics. A NZP-CSI$R S$-ResourceSet may either contain a set of configured CSI-RS or a set of SS blocks. Reporting of, for example, L1-RSRP for beam management can thus be based on measurements on either a set of SS blocks or a set of CSI-RS.

Note that the resource configuration is associated with a resource set. Measurements and corresponding reporting are thus in the general case carried out 
on a set of CSI-RS or a set of SS blocks. In the case of beam management, the resource set will typically consist of multiple CSI-RS, alternatively multiple SS blocks, where in practice each CSI-RS or SS block is associated with a specific beam. The device measures on the set of signals within the resource set and reports the result to the network as input to the beam-management functionality.

In addition to the quantity to report and the set of resources to measure on, the report configuration also describes when and how the reporting should be carried out. Similar to CSI-RS transmission, device reporting can be periodic, semi-persistent, or aperiodic.

As the name suggests, periodic reporting is done with a certain configured periodicity. Periodic reporting is always done on the PUCCH physical channel. Thus, in the case of periodic reporting, the resource configuration also includes information about a periodically available $\mathrm{PUCCH}$ resource to be used for the reporting.

In the case of semi-persistent reporting, a device is configured with periodically occurring reporting instances in the same way as for periodic reporting. However, actual reporting can be activated and deactivated by means of MAC signalling.

Similar to periodic reporting, semi-persistent reporting can be done on a periodically assigned PUCCH resource. Alternatively, semi-persistent reporting can be done on a semi-persistently allocated PUSCH. The latter is typically used for larger reporting payloads.

Aperiodic reporting is explicitly triggered by means of Downlink Control Information (DCI) signalling, more specifically within a CSI-request field within the uplink scheduling grant (DCI format $0_{-} 1$ ). The DCI field may consist of up to 6 bits with each configured aperiodic report associated with a specific bit combination. Thus, up to 63 different aperiodic reports can be triggered.

Aperiodic reporting is always done on the scheduled PUSCH and thus requires an uplink scheduling grant. This is the reason why the triggering aperiodic reporting is only included in the uplink scheduling grant and not in other DCI formats. It should be noted that, in the case of aperiodic reporting, the report configuration could actually include multiple resource sets for channel measurements, each with its own set of reference signals (CSI-RS or SSB). Each resource set is associated with a specific value of the CSI-request field in the DCI. By means of the CSI request the network can, in this way, trigger the same type of reporting but based on different measurement resources. Note that the same could, in principle, have been done by configuring the device with multiple report configurations, where the different resource configurations would specify the same reporting configuration and report type but different measurement resources. 
Table 2.7: Possible combinations of measurement report type and measurement resource type

\begin{tabular}{|l|c|c|c|}
\hline \multirow{2}{*}{ Report type } & \multicolumn{3}{|c|}{ Resource type } \\
\cline { 2 - 4 } & Periodic & Semi-persistent & Aperiodic \\
\hline \hline Periodic & Yes & No & No \\
\hline Semi-persistent & Yes & Yes & No \\
\hline Aperiodic & Yes & Yes & Yes \\
\hline
\end{tabular}

Periodic, semi-persistent, and aperiodic reporting should not be mixed up with periodic, semi-persistent, and aperiodic CSI-RS as described in Section 2.1.3. As an example, aperiodic reporting and semi-persistent reporting could very well be based on measurements on periodic CSI-RS. On the other hand, periodic reporting can only be based on measurements on periodic CSI-RS but not on aperiodic and semi-persistent CSI-RS. Table 2.7 summarises the allowed combinations of reporting type (periodic, semi-persistent, and aperiodic) and resource type (periodic, semi-persistent, and aperiodic).

\subsubsection{UE positioning}

In NR, the network can instruct the UE to start reporting its current location. A UE may, after being requested by the upper layers, send a Location measurement indication message to indicate the beginning or the conclusion of a location procedure by the UE. Before starting the procedure, the network should have notified the UE about the availability of measurement gaps the UE can use to obtain the location information. The UE shall complete the location procedure within the time allocated in the measurement gaps. If the allocated time is not enough to complete the location procedure, the UE will not trigger its location procedure. By sending this message, the UE notifies the network that it is going to start or stop location measurements which require measurement gaps.

\subsection{Conclusions}

The inclusion of high frequency transmissions demanded the introduction of important modifications in the PHY and upper layers of NR. The new standard provides the necessary technologies and mechanisms to enable an efficient management of transmission and reception beams. Beam management in NR is based on beam measurements of RS for downlink, uplink or both. This chapter of the Thesis has focused on measuring downlink RSs, which offers the 
possibility of measuring the directional transmission of SSBs used for initial access for beam management for free, without increasing the signalling overhead. Additionally, beam measurements can be done on CSI-RS resources.

There is, however, a clear lack of a precise beam management procedure in the $3 \mathrm{GPP}$ specification documents. It is, therefore, up to equipment manufacturers and operators to implement their own beam management algorithms. 



\section{Chapter 3}

\section{System Modelling}

This Thesis is oriented towards the study of beam tracking strategies within the ambit of the New Radio (NR) specifications defined by Third Generation Partnership Project (3GPP). In order to perform any assessment, it is necessary to have a deep knowledge of the system protocol under study before defining an evaluation methodology. This chapter of the Thesis deals with the latter issue.

There is an evident lack of real Millimeter Wave (mmWave) measurement data of users in mobility that can help research the beam tracking problem. For this reason, channel data must be artificially handcrafted. Good channel models providing spatial consistency are required.

This chapter of the Thesis is composed of two differentiated parts. The first part is devoted to providing the details of two channel models for large frequencies which were considered in this Thesis. The second part discusses the simulation tool used, focusing on the different implementation aspects which were necessary to implement the beam management framework.

This Chapter is structured as follows:

- Section 3.1 presents the point-to-point hardware configuration assumed in this Thesis .

- Section 3.2 introduces the realistic channel models used together with the network architecture to model the mmWave Fifth Generation (5G) system. Special attention is paid to the ray tracing channel model for mmWave communications created specifically for this Thesis. The main novelty of this ray tracing tool is that it runs with a video game engine.

- Section 3.3 addresses the problem of the generation of beams in the azimuth and elevation domain. This Thesis proposes an adaptation of Two 

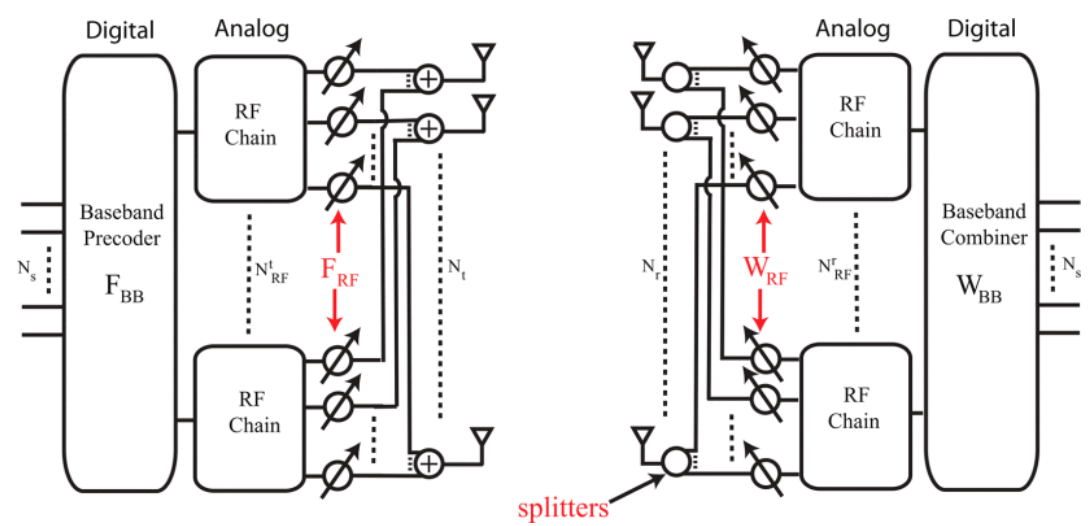

Figure 3.1: System model.

Dimension (2D) Discrete Fourier Transform (DFT) codebooks to support Three Dimension (3D) beamforming.

- Section 3.4 concludes the present chapter by highlighting the most significant revelations.

\subsection{Network architecture}

This Thesis assumes a point-to-point mmWave system as illustrated in Figure 3.1. This architecture is similar to works on mmWave Multiple-Input Multiple-Output (MIMO) communications [44, 55, 92, 93]. On one side, the Base Station (BS) is equipped with $N_{\mathrm{t}}$ antenna elements. On the other side of the link, a User Equipment (UE) is equipped with an antenna array of $N_{\mathrm{r}}$ antenna elements. The BS is capable of transmitting $N_{\mathrm{S}}$ data streams simultaneously through $N_{\mathrm{RF}}^{\mathrm{t}}$ Radio Frequency (RF) transmission chains, in such a way that $N_{\mathrm{S}} \leq N_{\mathrm{RF}}^{\mathrm{t}} \leq N_{\mathrm{t}}$. Similarly, the UE is equipped with $N_{\mathrm{RF}}^{\mathrm{r}} \mathrm{RF}$ chains to receive $N_{\mathrm{s}}$ simultaneous flows only if $N_{\mathrm{s}} \leq N_{\mathrm{RF}}^{\mathrm{r}} \leq N_{\mathrm{r}}$.

The number of simultaneous streams depends on $N_{\mathrm{RF}}$. In analog beamforming architectures, $N_{\mathrm{RF}}^{\mathrm{t}}=1$ and only one stream can be transmitted. In fully-digital beamforming, the number of transmission chains matches the number of antenna elements, so the number of simultaneous streams can be up to the number of antennas. The middle term is the hybrid beamforming architectures in which the number of flows matches the number of transmission chains. 
The considered transmitter architecture consists on a base band precoder (digital) $\mathbf{F}_{\mathrm{BB}}$ which is a $N_{\mathrm{RF}}^{\mathrm{t}} \times N_{\mathrm{S}}$ matrix, followed by an analog beamformer or RF precoder $\mathbf{F}_{\mathrm{RF}}$ which is a $N_{\mathrm{t}} \times N_{\mathrm{RF}}^{\mathrm{t}}$ matrix. The analog beamforming is composed of phase shifters, which implies that $\mathbf{F}_{\mathrm{RF}}$ has norm 1. Note that the phase shifters are all connected to all the antenna elements of the array.

The discrete-time signal transmitted can be written as:

$$
\mathbf{x}=\mathbf{F}_{\mathrm{RF}} \mathbf{F}_{\mathrm{BB}} \mathbf{S}
$$

where $\mathbf{s}$ is the $N_{\mathrm{S}} \times 1$ input signal vector representation, which energy is distributed as $\mathbb{E}\left\|\mathbf{s s}^{*}\right\|^{2}=\frac{1}{N_{\mathrm{s}}} \mathbf{I}_{N_{\mathrm{s}}}$ and $\mathbf{x}$ is the $N_{\mathrm{t}} \times 1$ vector representing the signal transmitted by the BS.

Assuming a narrow-band block fading channel as in [55], the received signal can be expressed as follows:

$$
\mathbf{y}=\sqrt{\rho} \mathbf{H F}_{\mathrm{RF}} \mathbf{F}_{\mathrm{BB}} \mathbf{s}+\mathbf{n}
$$

where $\mathbf{y}$ is the signal vector $N_{\mathrm{r}} \times 1$ arriving at the $\mathrm{UE}, \mathbf{H}$ is the $N_{\mathrm{r}} \times N_{\mathrm{t}}$ channel matrix, $\rho$ is a parameter describing the received power level, and $\mathbf{n}$ is the i.i.d. noise vector of size $N_{\mathrm{r}} \times 1$. The channel matrix satisfies that $\mathbb{E}\left[\|\mathbf{H}\|_{F}^{2}\right]=N_{\mathrm{t}} N_{\mathrm{r}}$

The recovered signal at the end of the analog beamformers and digital combiner stages can be written as:

$$
\tilde{\mathbf{s}}=\sqrt{\rho} \mathbf{W}_{\mathrm{BB}}^{*} \mathbf{W}_{\mathrm{RF}}^{*} \mathbf{H} \mathbf{F}_{\mathrm{RF}} \mathbf{F}_{\mathrm{BB}} \mathbf{s}+\mathbf{W}_{\mathrm{BB}}^{*} \mathbf{W}_{\mathrm{RF}}^{*} \mathbf{n}
$$

where $\mathbf{W}_{\mathrm{BB}}$ is the baseband combining matrix of size $N_{\mathrm{RF}}^{\mathrm{r}} \times N_{\mathrm{s}}$ and $\mathbf{W}_{\mathrm{RF}}$ is the analog beamforming matrix of size $N_{\mathrm{r}} \times N_{\mathrm{RF}}^{\mathrm{r}}$.

For the sake of simplicity and without loss of generality, this Thesis will consider analog beamforming with one RF chain at both communications sides. This simplifies Equation 3.1, Equation 3.2 and Equation 3.3 by reducing the precoding and combining matrices to 1 , which can be then rewritten as:

$$
\begin{gathered}
\mathbf{x}=\mathbf{F}_{\mathrm{RF}} \mathbf{s} \\
\mathbf{y}=\sqrt{\rho} \mathbf{H F}_{\mathrm{RF}} \mathbf{s}+\mathbf{n} \\
\tilde{\mathbf{s}}=\sqrt{\rho} \mathbf{W}_{\mathrm{RF}}^{*} \mathbf{H} \mathbf{F}_{\mathrm{RF}} \mathbf{s}+\mathbf{W}_{\mathrm{RF}}^{*} \mathbf{n}
\end{gathered}
$$

Furthermore, $\mathbf{F}_{\mathrm{RF}}$ and $\mathbf{W}_{\mathrm{RF}}$ matrices are now beamforming vectors of $N_{\mathrm{t}} \times 1$ and $N_{\mathrm{r}} \times 1$ respectively. 
The mmWave frequency bands are known to suffer high free-space propagation losses, which leads to poor scattering environments, with only few substantial contributions [23]. The traditional geometric channel models used in sub $6 \mathrm{GHz}$ MIMO systems are inaccurate for sparse scattering environments, as occurs in mmWave transmissions [55]. For this Thesis, a narrowband clustered channel model based on Saleh-Valenzuela model [94] is assumed, in alignment with other relevant research works in mmWave MIMO systems [44, 55, 92, 93]. This channel model assumes that the multi-path environment is composed by $l$ scattering clusters, and each one of them originates $i$ rays which are slightly different one from the others. The general MIMO channel matrix $\mathbf{H}$ can be written as follows:

$$
\mathbf{H}=\gamma \sum_{i, \ell} \alpha_{i \ell} \Lambda_{\mathrm{r}}\left(\phi_{i \ell}^{\mathrm{r}}, \theta_{i \ell}^{\mathrm{r}}\right) \Lambda_{\mathrm{t}}\left(\phi_{i \ell}^{\mathrm{t}}, \theta_{i \ell}^{\mathrm{t}}\right) \mathrm{a}_{\mathrm{r}}\left(\phi_{i \ell}^{\mathrm{r}}, \theta_{i \ell}^{\mathrm{r}}\right) \mathrm{a}_{\mathrm{t}}\left(\phi_{i \ell}^{\mathrm{t}}, \theta_{i \ell}^{\mathrm{t}}\right)^{*}
$$

where $\gamma$ is a power normalisation factor which depends on the number of transmit and receive antennas and the total number of rays involved, such as $\gamma=\sqrt{\frac{N_{\mathrm{t}} N_{\mathrm{r}}}{N_{\text {rays }}}}, \alpha_{i \ell}$ is the complex path gain of the $i$-th ray belonging to the $l$-th cluster. Since the channel model is three-dimensional, the Angle of Departure (AoD) and Angle of Arrival (AoA) are expressed in spherical coordinates, that is, providing the azimuth $(\phi)$ and elevation $(\theta)$ angles. $\phi_{i \ell}^{\mathrm{t}}$ and $\theta_{i \ell}^{\mathrm{t}}$ correspond to the azimuth and elevation AoD. Similarly, $\phi_{i \ell}^{\mathrm{r}}$ and $\theta_{i \ell}^{\mathrm{r}}$ refer to the azimuth and elevation AoA.

Functions $\Lambda_{\mathrm{t}}\left(\phi_{i \ell}^{\mathrm{t}}, \theta_{i \ell}^{\mathrm{t}}\right), \Lambda_{\mathrm{r}}\left(\phi_{i \ell}^{\mathrm{r}}, \theta_{i \ell}^{\mathrm{r}}\right)$ represent the antenna element of the BS and UE, given the AoD and AoA. Typically, antenna elements at UEs are omnidirectional, meaning that they radiate in all directions equally. On the contrary, antenna elements at the base station have an angular response in order to create the sectors which are typical in macro-cellular deployments. 3GPP provides in its channel model Technical Report (TR) the reference antenna element radiation diagram at the $\mathrm{BS}$ for model calibration [6].

The BS and UE array response vectors, $\mathbf{a}_{\mathrm{t}}$ and $\mathbf{a}_{\mathrm{r}}$ respectively, are a function of the antenna array geometry and frequency. The MIMO channel equation takes arbitrary antenna array configurations. A widely-used antenna array configuration is Uniform Linear Array (ULA), which is illustrated in Figure 3.2(a). Assuming that $N$ antenna elements are placed over the $x$-axis, the ULA response equation is as follows:

$$
\mathbf{a}_{\mathrm{ULA}}(\phi)=\frac{1}{\sqrt{N}}\left[1, e^{j k d \sin (\phi)}, \ldots, e^{j(N-1) k d \sin (\phi)}\right]^{T},
$$

where the wavenumber $k=\frac{2 \pi}{\lambda}$, being $\lambda$ the wavelength, and $d$ is the physical separation between adjacent antenna elements. 


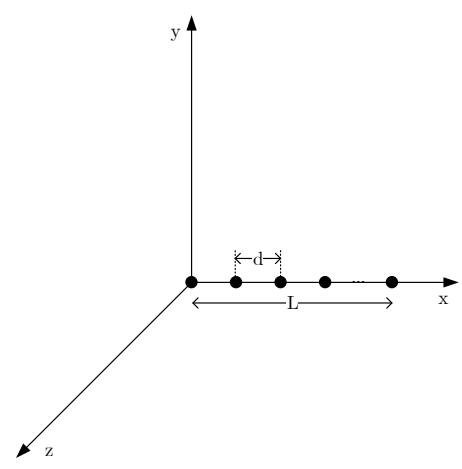

(a) Uniform Linear Array

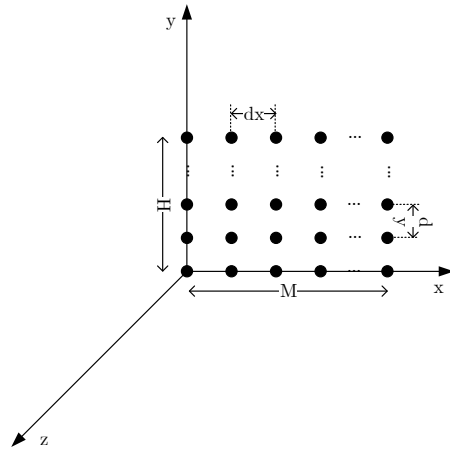

(b) Uniform Rectangular Array

Figure 3.2: Antenna array configurations

Note that ULA response equation only depends on azimuth angles, and it cannot fully capture the multipath components in elevation. Such response is impractical for 3D beamforming, which is of interest in this Thesis work. 3D beamforming can be achieved with an antenna array which radiation elements are placed over the same plane. This Thesis will focus on Uniform Rectangular Array (URA). The antenna array response equation for an URA on the $x y$-plane, with $W$ antenna elements in the horizontal plane and $H$ antenna elements in the vertical plane is:

$$
\begin{aligned}
\operatorname{a}_{\mathrm{URA}}(\phi, \theta)= & \frac{1}{\sqrt{N}}\left[1, \ldots, e^{j k d(m \sin (\phi) \sin (\theta)+n \cos (\theta))}, \ldots,\right. \\
& \left.e^{j k d((W-1) \sin (\phi) \sin (\theta)+(H-1) \cos (\theta))}\right]^{T}
\end{aligned}
$$

where $m$ and $n$ are the antenna element index of the horizontal and vertical planes, respectively, $0 \leq m<W, 0 \leq n<H$ and $N=W H$.

\subsection{Channel models for mmWave}

\subsubsection{GPP channel model for frequencies over $6 \mathrm{GHz}$}

On December 2016, 3GPP published its standard document TR 38.900 containing the reference channel model for evaluating $5 \mathrm{G}$ systems and technologies 
operating in high frequency bands, up to $100 \mathrm{GHz}$ [5]. The channel model is the result of the natural evolution of the 3D statistical spatial channel model used in Long Term Evolution (LTE) [19]. The parameters of the new channel model were obtained from exhaustive propagation measurement campaigns at mmWave frequencies conducted by several 3GPP partners. The final channel model document, including also sub $6 \mathrm{GHz}$ channels, is included in TR 38.901 [6].

The maximum system bandwidth allowed represents the $10 \%$ of the selected carrier frequency, with a practical upper bound of $2 \mathrm{GHz}$. The model supports the mobility of one of the two communication edges. The modular design of the channel model allows adding optional features into the base model. One interesting addition in mobility scenarios is the calculation of the spatial consistency of the channel realisations. With spatial consistency, 3GPP model ensures that the channel realisation among very-close users are correlated and not completely independent. Other interesting additions to the channel are blockage modelling or User Terminal (UT) rotation.

$3 \mathrm{GPP}$ channel model for frequencies over This model supports different scenarios, which must be chosen when setting the simulations parameters:

- Urban Micro-cell (UMi) assumes small base stations with small Inter-Site Distance (ISD) among cells, which targets at densely deployed urban area. This scenario also assumes a street canyon scenario where the BS are at street level, not higher than building rooftops, hence blocking signals from cells in surrounding streets. UMi scenario considers outdoor, Outdoorto-Indoor (O2I) and Outdoor-to-Outdoor (O2O) propagation conditions.

- Urban Macro-cell (UMa) is similar to UMi but BSs are place on rooftops and the ISD is larger.

- Rural Macro-cell (RMa) focuses on a rural deployment or low-density population scenarios. The coverage area of cells are larger than the in the previous two urban cases, and takes typical ISD values of thousands of meters.

- Indoor Office (In) aims at capturing the rapid channel condition fluctuation which are typical in offices, with cubicles and wireless access points on ceilings, and shopping malls, which might have several stories with open areas shared by different floors.

The channel model coefficients generation is illustrated in Figure 3.3. Given a certain scenario, the $3 \mathrm{D}$ positions, velocities and indoor/outdoor state of UT and BS, the model assigns a Line of Sight (LoS)/Non Line of Sight (NLoS) condition to each link according to scenario-specific probability distributions. This 


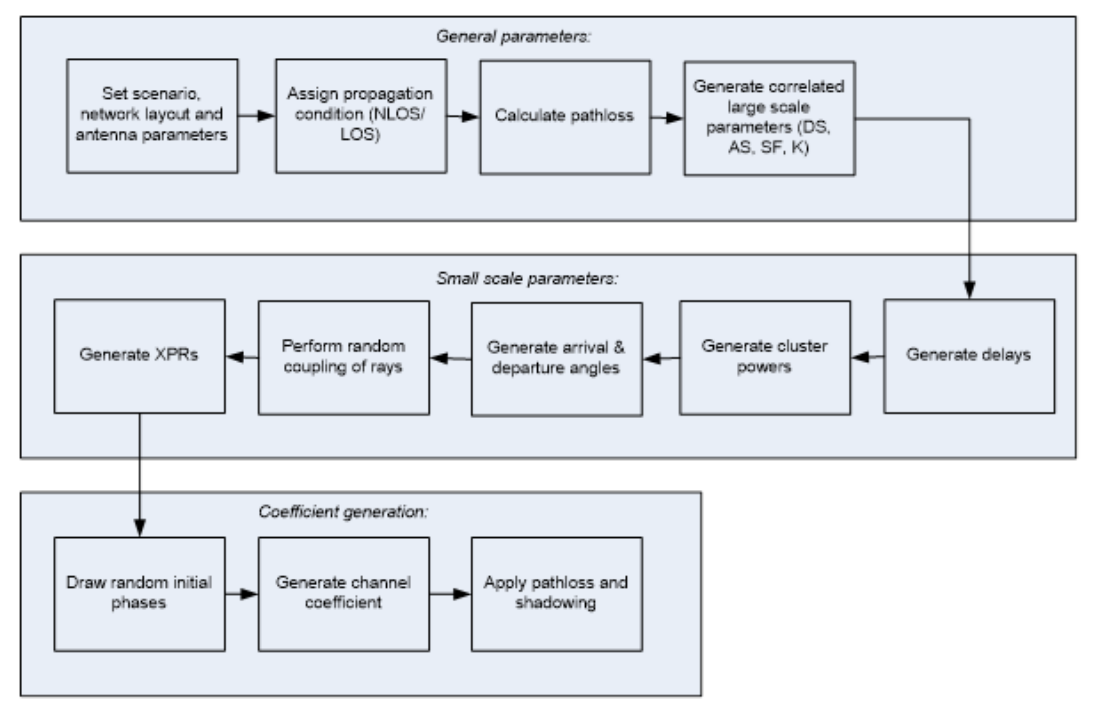

Figure 3.3: The different channel coefficients are generated sequentially following this block diagram [6]

condition affects both the pathloss computation and the generation of fading parameters. For the pathloss, different formulas are provided, according to the scenario, and an additional O2I component can be added, with the possibility of modelling different kinds of building penetration losses (glass,concrete, wood).

The channel between a transmitter point and a receiver end is described by a channel matrix $\mathbf{H}(t, \tau)$ of size $N_{r x} \times N_{t x}$, where $N_{r x}$ and $N_{t x}$ are the number of antennas at the receiver and the transmitter, respectively. Each entry depends on $N$ different multipath components, named clusters, which have different delays and received powers, following an exponential Packet Data Protocol (PDP). The clusters are themselves a combination of $M$ rays, each one with small deviations from the others in terms of the arrival and departure angles in both vertical and horizontal planes. All these parameters are randomly generated from the distributions that take different simulation parameters, naming the scenario, the LoS condition, the presence of indoor or outdoor transitions, the carrier frequency and bandwidth, the 2D and 3D distance between transmitter and receiver, and their respective antenna configurations and heights. The model distinguishes large scale and slow scale fading components. The large scale fading depends on user mobility or changes in the propagation conditions in the scenario, that have an impact on parameters such as the Delay 
Spread (DS), the angular spread of arrival and departure, either in the azimuth, i.e., the horizontal plane (ASA, ASD), or in the elevation plane or zenith (ZSA, ZSD), the Ricean factor for the LoS condition $(K)$ or the shadowing. The large scale parameters for each link are generated and cross-correlated using the procedure originally suggested in the WINNER-II channel model [32]. Different correlation parameters are given for the scenarios previously introduced and for the LoS and NLoS conditions. The small scale fading, instead, depends on the small variation of the multipath components within a cluster, including the arrival and departure angles of the rays, small power variations, and the Doppler effect in mobility scenarios. The main difference between a LoS and a NLoS channel is the overall reduced power levels in the clusters in NLoS.

The channel model for NR specifies additional modelling components that can be included to the base simulator depending on the level of detail needed for the simulations. For example, in a drop-based simulation with no mobility, the basic channel model is recommended. But, if the simulation requires user mobility, more realistic results are obtained from a channel model that updates the channel matrix correlating it with the channel conditions in the previous location of the user. This can be achieved with the spatial consistency component, which provides the decorrelation distances for the given fading parameters, the LoS condition whether the UT is outdoor or indoor. The blockage option introduces an attenuation factor over the received power of certain clusters according to some randomly deployed blockers simulating the presence of human bodies, vehicles, etc. Another interesting feature in mobility scenarios is the addition of the UT rotation. The UT orientation is critical when applying beamforming in the UT, where beams are referred to the antenna array plane. Another optional feature is oxygen absorption,that introduces a frequency-dependent additional pathloss factor to account for the atmospheric absorption at certain frequencies.

The 3GPP channel model may also interact with an external ray tracing tool in order to obtain the ray directions from a virtually-recreated scenario and deterministically create the different clusters. This approach is referred to as map-based hybrid channel model and it is clearly influenced by the METIS project activity in redefining statistical channel models for $5 \mathrm{G}$ networks needs [30]. The flow explaining the channel coefficient calculation in the map-based hybrid channel with ray information from external ray tracer is illustrated in Figure 3.4.

\subsubsection{Ray tracing tool in Unity}

Professional ray tracing software such Remcom's InSite [72] are sophisticated tools with an elevated degree of accuracy in their channel propagation predic- 


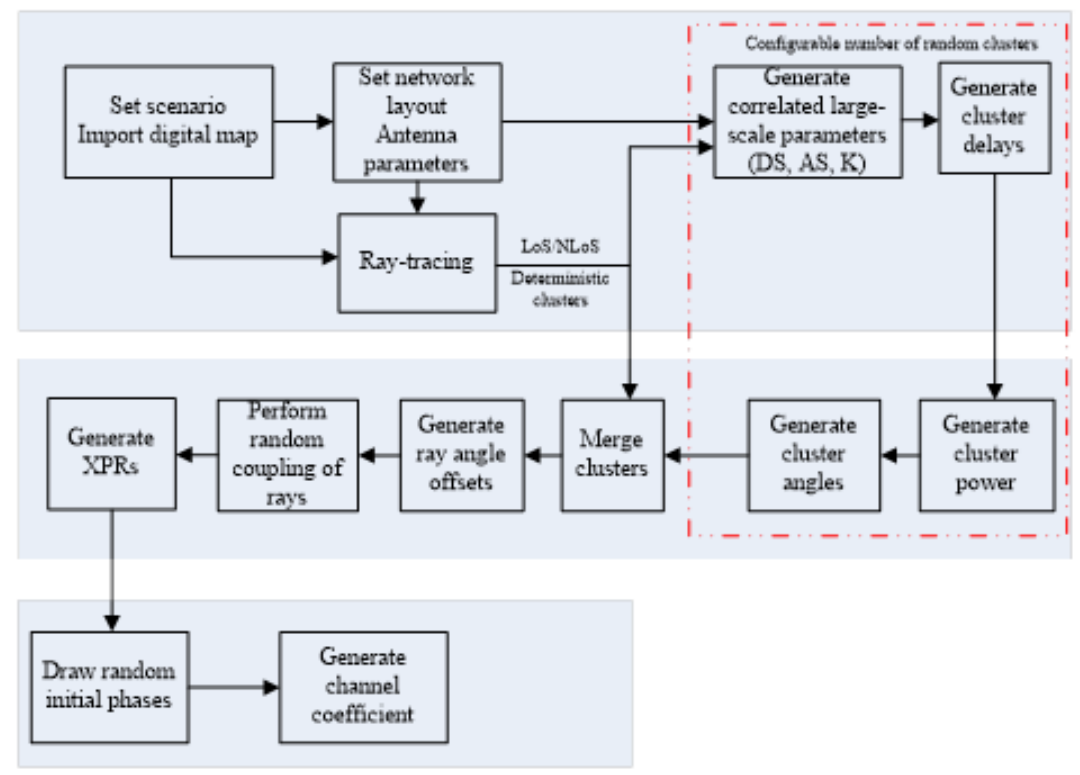

Figure 3.4: Ray information, including arriving and departure angles, delays and propagation losses are calculated with an external raytracing tool and used in the creation of the channel coefficients [6].

tions. This kind of software is computationally very intensive and calculating the propagation conditions in a relatively small are takes a long time to complete. In order to alleviate the computational burden, calculations can be run in paralleled in multiple General-Purpose Graphics Processing Unit (GPGPU). The use of open-source video game engines like Unity is an attractive alternative given the collection of optimised library to accelerate ray tracing calculations.

\section{Creating the 3D model}

The first issue to solve is how to create a 3D model of a real-world area. There are several possible approaches for the creation of a virtual scenario. Synthetic scenarios devised for mobile technology testing purposes are typically a simple set of regular polygons spread over a surface, which can be recreated in Unity easily. However, when it is needed to realistically reproduce a real-world area with many irregular shapes, then the use of a 3D modelling software is required.

Obtaining realistic 3D models of urban environments is a time-consuming process if the recreation has to be done manually by a team of digital artists, 


\section{CHAPTER 3. SYSTEM MODELLING}

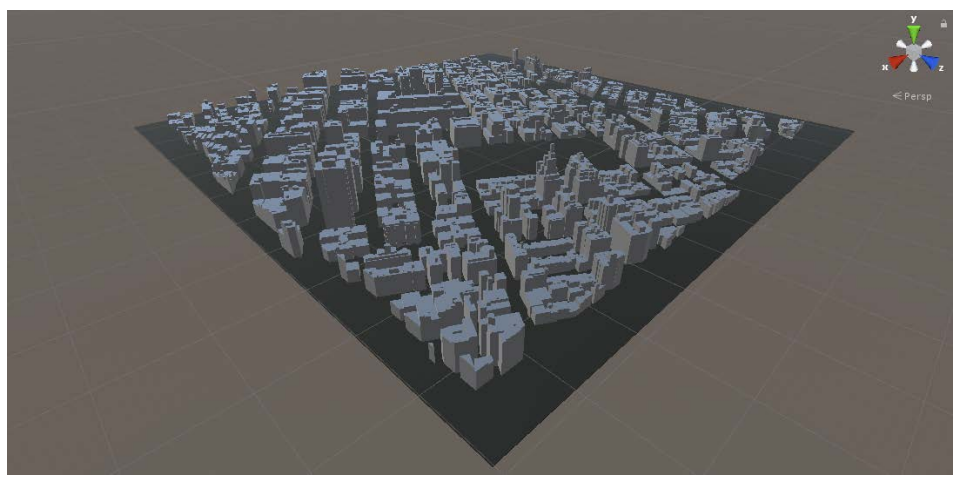

Figure 3.5: A panoramic view of the Downtown Manhattan 3D model recreated in Unity.

and it would be largely desirable to add some degree of automation in the creation process. Luckily, when the interest is on recreating scenarios from the real world, the process can be alleviated by using geographical data sources and integrating the resulting models in the game engine directly [95]. The use of geomatics engineering databases like Open Street Map (OSM) [96] or Geographical Information Service (GIS) may boost the modelling design process by introducing automations whilst making the resulting model geographically consistent [97]. OSM is a valid free open-source option, which is basically supported by the altruistic work of the OSM community. The main drawback of this open-source database is the incompletion or total absence of some regional data [98]. On the other hand, GIS databases are typically commercialised and, therefore, shall contain complete information of a given geographical area and may provide realistic characterisations in computer-based simulations [97].

The virtual scenario recreated in Figure 3.5 is a spatially-consistent threedimensional model of a large area in Downtown Manhattan in New York. In order to obtain the 3D model, we imported the geographical information in Autodesk 3ds $\operatorname{Max}^{\circledR}$ and exported the model in Filmbox (FBX ${ }^{\circledR}$ ) format, which is completely compatible with Unity. The resulting model accurately reproduced the shape of the buildings, although some post-processing exercises was required to add architectural elements such as trees, billboards, lamp posts, traffic lights, and so on. 


\section{Description of the ray tracing tool}

The ray tracing channel model created in Unity for this Thesis was inspired by the dissertation in [99]. This method calculates the propagation between a transmitter point and a receiver point in the $3 \mathrm{D}$ space. The ray tracing technique used is ray launching, which is describes as follows. The transmitter launches a ray in a given direction, seeking to hit the receiver's surface. A sphere with an arbitrary radius is placed at the receiver point and only the rays hitting this sphere are considered in the calculation of the electric field at the receiver location. Different rays are launched in separated time intervals, sweeping the whole 3D space. The angular resolution is configurable in azimuth $(\theta)$ and elevation $(\phi)$. The number of reflections is also configurable. However, the contribution of rays with more than 3 reflections is negligible [42] and discarded in order to reduce the computational burden.

The ray tracing algorithm in Unity calculates the signal power level at the measurement point. Since the urban channels are typically multi-path, the resulting power level is the result of the sum of each arriving ray. Accordingly, the power level at the measurement point is the addition of the Free Space Power Loss (FSPL) of the direct ray in case of LoS condition and the reflected rays.

According to ITU recommendation P.525-2 [100], the FSPL can be obtained as follows:

$$
L_{F S P L}(d B)=32.4+20 \log f+d
$$

where $f$ is the frequency of the transmitted wave in $\mathrm{MHz}$ and $d$ is the distance between transmitter and receiver expressed in kilometres.

To calculate the power level of a reflected signal, it is necessary to operate in the electric field $E$. In free-space propagation, the conversion from the transmitted power to field strength is defined as follows:

$$
E=P_{t}-20 \log d+74.8
$$

where $P_{t}$ is the isotropic transmit power expressed in $\mathrm{dBW}$ and electric field strength $E$ is expressed in $\mathrm{dB} \mu \mathrm{V} / \mathrm{m}$ units. On the other hand, the power received with an isotropic antenna for a given electrical field $E$ is

$$
P_{r}=E-20 \log f+167.2
$$

Then, the resulting $L_{F S P L}$ in terms of $E$ can be rewritten as follows:

$$
L_{F S P L}=P_{t}-P_{r}=P_{t}-E+20 \log f+20 \log d+167.2
$$


The electric field is a vector which is composed of two components, parallel $\hat{e}_{\|}$and perpendicular $\hat{e}_{\perp}$ to the plane of incidence.

Considering an electromagnetic wave impinging on a surface at point $\mathbf{Q}$, the electric field of the reflected wave is determined by the reflection coefficient matrix $\mathbf{R}$.

$$
\mathbf{E}_{r}(Q)=\mathbf{R E}_{i}(Q)
$$

where $\mathbf{E}_{r}(Q)$ is the electric field of the reflected wave and $\mathbf{E}_{i}(Q)$ is the electric field of the impinging wave at point $Q$. Note that both impinging and reflected electric fields are vectors, and $\mathbf{R}$ is a matrix.

The reflection coefficient $\mathbf{R}$ can also be decomposed into two components parallel and perpendicular to the incidence plane, denoted as $R_{\|}$and $\hat{e}_{\perp}$, respectively, which are defined as follows:

$$
\begin{aligned}
R_{\perp} & =\frac{\eta_{2} \cos \theta_{t}-\eta_{1} \cos \theta_{i}}{\eta_{2} \cos \theta_{t}+\eta_{1} \cos \theta_{i}} \\
R_{\|} & =\frac{\eta_{2} \cos \theta_{i}-\eta_{1} \cos \theta_{t}}{\eta_{2} \cos \theta_{i}+\eta_{1} \cos \theta_{t}}
\end{aligned}
$$

where $\eta_{1}$ and $\eta_{2}$ denotes the wave impedance in free space (vacuum) and the medium of the surface, thet $a_{i}$ is the angle of the incident ray with respect to the normal direction of the plane and the thet $a_{t}$ is the angle of the transmitted ray with respect to the normal direction of the plane. The wave impedance $\eta$ is expressed as follows

$$
\eta=\sqrt{\frac{j \omega \mu}{\sigma+j \omega \epsilon}}
$$

where $j$ is the imaginary unit, and $\omega$ is the angular frequency of the wave, $\mu$ is the magnetic permeability, $\sigma$ is the electrical conductivity of the medium, and $\epsilon$ is the real part of the electric permitivity.

Equation 3.14 can be separated in the parallel and perpendicular components to the incident plane:

$$
\begin{gathered}
E_{r \|}=R_{\|} E_{i \|} \\
E_{r \perp}=R_{\perp} E_{i \perp}
\end{gathered}
$$

The magnitude of the electric field after the reflection can be obtained as

$$
|E|=\sqrt{\left|E_{r \|}\right|^{2}+\left|E_{r \perp}\right|^{2}}
$$


The multi-path channel response is typically represented with the PDP, which represents the intensity signal received through rays or tabs in time. Different tabs arrive in different time instants. This time delay between contributions depends on the difference on the distance travelled by the waves.

The Unity ray-tracer is capable of estimating the channel propagation conditions in a given area of interest. It is also possible to define a set of points of interest on which ray tracing calculations are to be performed. This characteristic allows creating user walks, which can be used in mobility models.

The ray tracing tool in Unity stores the channel information at the measurement points in a log file. This log file contains the PDP information, including the power level and the time delay, the AoD and AoA separated in azimuth and elevation planes in order to support $3 \mathrm{D}$ channel modelling. This channel information file, in the appropriate format, can be used by simulators recreating realistic wave propagation conditions.

\subsection{Codebook-based beamforming}

In a mmWave NR system, the transmission of the Synchronization Signal (SS) described in Section 2.1.2 is beamformed and, consequently, SS/Physical Broadcast Channel (PBCH)-Block (SSB)s are sequentially transmitted on different beams. This procedure is referred to as beam sweeping.

Beam sweeping is a blind process in which the NR base station (gNB), i.e. the 5G BS, transmits Reference Signal (RS) without any prior knowledge of the channel status perceived by UEs. For this reason, beams are obtained out of a predefined matrix of beam forming vectors known as codebook. A codebook is composed by codewords, and each codeword determines the weights applied to each antenna element phase shifter to conform the beam.

This Thesis work aims at covering as much surface as possible with equallydistributed beams. DFT codebooks [101] are a perfect fit for such objective, and have been massively used in the MIMO systems literature [102, 103]. Some of the properties of DFT codebooks assuming a ULA are the following: beams are spread almost proportionally over a circumference and the beamwidth or HalfPower Beamwidth (HPBW) scales down with the number of antenna elements and increases the beam gain.

The DFT beamforming codebook matrix $\mathbf{C}$ is the concatenation of several codewords $\mathbf{c}_{\mathbf{m}}: \mathbf{C}=\left\{\mathbf{c}_{0}, \mathbf{c}_{1}, \cdots, \mathbf{c}_{N}\right\}$, where $N$ is the number of codewords of the codebook and $m=0,1, \cdots, N$.

A DFT codeword $\mathbf{c}_{\mathbf{m}}$ is expressed as follows: 


$$
\mathbf{c}_{\mathbf{m}}=\frac{1}{\sqrt{N_{t}}}\left[1, \exp \frac{j 2 \pi m}{N}, \ldots, \exp \frac{j 2 \pi\left(N_{t}-1\right) m}{N}\right]^{T}
$$

where $N_{t}$ is the number of antenna elements of the ULA.

It is worth noting that ULA beam diversity is restricted to the azimuth plane. There is no change of beams in elevation. Elevation or $3 \mathrm{D}$ beamforming is interesting not only to provide beam coverage to users in positions over the transmitting antenna, but to increase the beam gain of users from different distances away from the BS.

To enable 3D beamforming, antennas cannot be arranged over a single dimension in a straight line. To provide elevation gain the antennas must be placed on a plane. This Thesis work assumes URA with a symmetric antenna separation. However, the number of vertical and horizontal antenna elements does not necessarily need to be the same.

DFT codebooks cannot be directly applied to URA. This Thesis work adopts the Kronecker product approximation described in [102]. The rectangular antenna array is decomposed in a horizontal and a vertical ULAs with their respective DFT codebooks. This approximation assumes that there exists no interaction between the horizontal and vertical antenna elements. The final URA codebook matrix is the Kronecker product of the vertical and horizontal DFT codebooks as described in Equation 3.22.

$$
\begin{gathered}
\mathbf{c}_{m}^{(v)}=\frac{1}{\sqrt{N_{t}^{(v)}}}\left[1, \exp \frac{j 2 \pi m}{\beta N_{v}}, \ldots, \exp \frac{j 2 \pi\left(N_{t}^{(v)}-1\right) m}{\beta N_{v}}\right]^{T} \\
\mathbf{c}_{n}^{(h)}=\frac{1}{\sqrt{N_{t}^{(h)}}}\left[1, \exp \frac{j 2 \pi n}{N_{h}}, \ldots, \exp \frac{j 2 \pi\left(N_{t}^{(h)}-1\right) n}{N_{h}}\right]^{T} \\
\mathbf{c}_{N_{h} m+n}=\mathbf{c}_{m}^{(v)} \otimes \mathbf{c}_{n}^{(h)}
\end{gathered}
$$

where $N_{t}^{(h)}$ and $N_{t}^{(v)}$ are the number of antenna elements of the horizontal and vertical sub-arrays, and $N_{h}$ and $N_{v}$ are the number of codewords of the horizonal and vertical DFT codebooks, respectively. There is an additional parameter $\beta$ in the vertical codebook, which is a scaling factor associated with downtilt. In the literature, this codebook is known as Kronecker Product Codebook (KPC) and has $N_{v} \times N_{h}$ codewords: $\mathbf{C}=\left\{\mathbf{c}_{0}, \mathbf{c}_{1}, \cdots, \mathbf{c}_{N_{v} \times N_{h}-1}\right\}$

In the ambit of this Thesis, two antenna array configurations are going to be used. One is a rectangular antenna array of 64 omnidirectional radiation elements, with $W=16$ elements in the horizontal axis and $H=4$ in the 
vertical axis. The second configuration is another rectangular-shaped array of 16 omnidirectional radiation elements, with $W=8$ in the horizontal axis and $H=2$ in the vertical axis. The number of horizontal and vertical beams of each configuration matches their corresponding number of antenna elements in each domain. The downtilt parameter $\beta=1$ and the antenna element separation is half wavelength.

Each beam has a unique identification number. For simplicity, this identification number is referred to beam id. The codebook-based beamforming has a codebook matrix in which each column of the matrix is one codeword. And the codeword determines the weights and phases that feeds the antenna array to beamform. So, there is a direct codeword-beam relationship. In this Thesis, beam ids are given based in their position in the codebook matrix, this is, the column number. The Kronecker codebook in Equation 3.22 is composed of $H$ blocks, and each block is composed of the $W$ beams contained in the same azimuth plane. Therefore, it is possible to obtain the azimuth and elevation information from the beam id. Equations 3.23 and 3.24 determine the azimuth $b_{\mathrm{az}}$ and elevation beam $b_{\mathrm{el}}$ of a given beam id $b$ :

$$
\begin{gathered}
b_{\mathrm{az}}=b \bmod W \\
b_{\mathrm{el}}=\operatorname{floor}(b / W)
\end{gathered}
$$

where mod is the modulus operation function and floor is the round to next smaller integer function.

The opposite operation of determining the beam id $b$ from the azimuth and elevation beams is possible:

$$
b=W \cdot b_{\mathrm{el}}+b_{\mathrm{az}}
$$

The combined antenna array and codebook maximum gains are illustrated in Figure 3.6. The horizontal axis contains the angular variation in azimuth, ranging from $-180^{\circ}$ to $180^{\circ}$, whereas the vertical axis represents the variation on elevation angles, raging from $-90^{\circ}$ to $90^{\circ}$. Figure 3.6(a) illustrates the beam gains of the BS, whereas Figure 3.6(b) represents the beam gains of the UE. The figure represents for each combination of azimuth and elevation beams, the maximum beam gain obtained from all the beamforming vectors. As it can be seen, the number of lobes is larger on the BS and, as a result, the lobes are more compressed in the two angular domains than in the UE beam gain response. In addition, due to the larger number of antenna elements, the beam gains in the BS are larger than in the UE, with a peak value difference between the two antenna configurations of $10 \mathrm{~dB}$. 


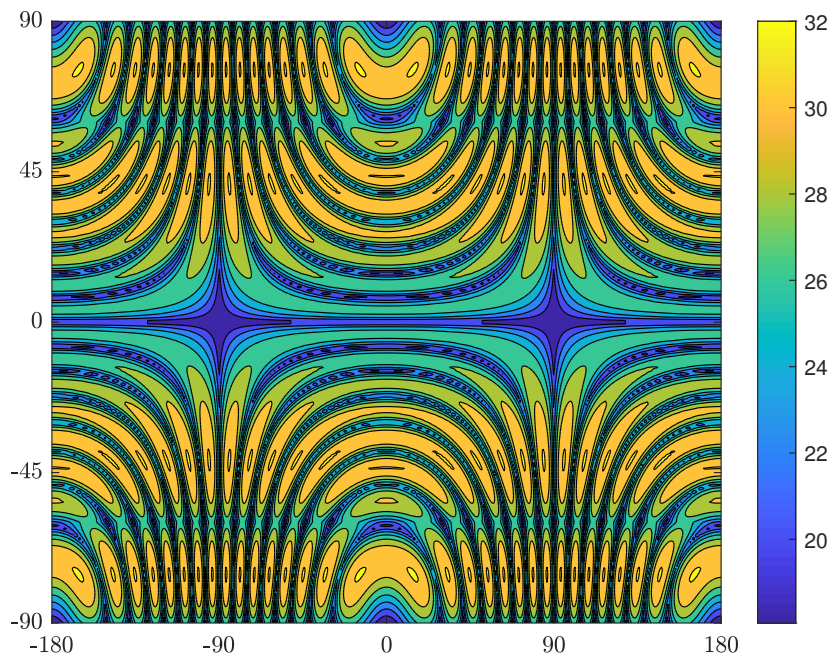

(a) $16 \times 4$ URA

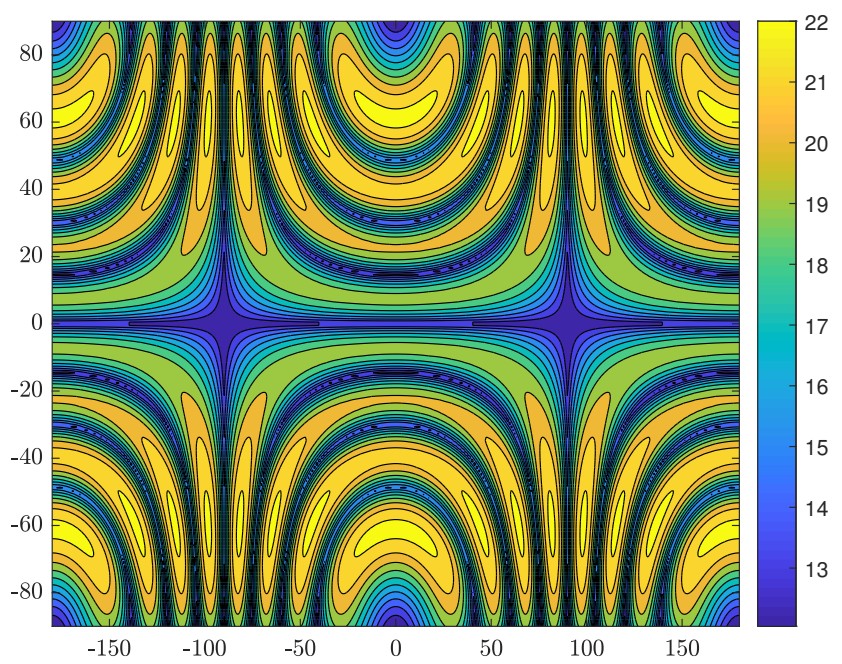

(b) $8 \times 2$ URA

Figure 3.6: Maximum beam gains (dB) varies in azimuth (horizontal axis) and elevation (vertical axis) angles due to the combined effect of the antenna array and the Kronecker codebook. 


\subsection{Conclusions}

This chapter has presented the system model assumed in the Thesis to evaluate the beam tracking strategies. The Thesis has presented the MIMO architecture for MIMO point-to-point communications in the mmWave band.

This chapter has introduced a ray tracing tool to estimate the signal propagation in the mmWave band. This tool has the particularity of being implemented for Unity video game development platform, which can take advantage of multiple GPGPU to speed up calculations. In combination with the visualisation platform described in Appendix A, this ray tracing tool allows obtaining realistic channel estimations of any real scenario virtually recreated. In the right format, the channel characterisation can be used in any external simulator.

Then, the general description of the channel model was adapted to analog transceivers, which is of particular interest in this Thesis. Special attention was put to enabling $3 \mathrm{D}$ beamforming, in such a way that beams are diverse in azimuth and also in elevation. To that aim, a special version of the DFT codebook, the Kronecker codebook, was discussed. Considering the substantial gains provided by the combination of the antenna array response and the Kronecker codebook, directional communications in the mmWave band are certainly feasible from an analytical point of view. 



\section{Chapter 4}

\section{Simulation Framework and Visualisation}

The objective of this Thesis is the proposal and evaluation of beam tracking strategies within the ambit of the New Radio (NR) specifications defined by Third Generation Partnership Project (3GPP). This evaluation is to be achieved by means of computer simulations. Once the system model has been declared in Chapter 3, this chapter of the Thesis introduces the used simulation tool and describes the modifications made in that tool in order to implement the beam management framework, which serves as a basis to the devised beam tracking strategies.

The present Chapter of the Thesis is structured in the following way:

- Section 4.1 introduces the reader to the simulation tool ns-3 and to the Millimeter Wave (mmWave) module for Fifth Generation (5G) end-toend network simulations assessment.

- Section 4.2 discusses the necessary modifications to adapt the mmWave module for ns-3 to the Release $15 \mathrm{NR}$ specifications and provides the implementation details of the beam management framework.

- Section 4.3 presents some results from simple ns-3 simulation runs that serve to prove that beam management effectively manages the mmWave beams.

- Section 4.4 illustrates through some visualisation examples that there exist alternative ways to efficiently communicate and educate on $5 \mathrm{G}$ technologies. 
- Section 4.5 list the main conclusions drawn from the experience with ns-3 and the beam management framework developed for this Thesis.

\subsection{End-to-end network simulation with ns-3}

ns-3 is a discrete event network simulator for research on fixed and wireless networks. ns-3 has been developed to provide an open, extensible network simulation platform, for networking research and education. In brief, ns-3 provides models of how packet data networks work and perform, and provides a simulation engine for users to conduct simulation experiments. Some of the reasons to use ns-3 include to perform studies that are more difficult or not possible to perform with real systems, to study system behaviour in a highly controlled, reproducible environment, and to learn about how networks work. Even if ns-3 focuses on modelling how Internet protocols and networks work, non-Internet-based systems can also be simulated.

The ns-3 project is committed to building a solid simulation core that is well documented, easy to use and debug, and that caters to the needs of the entire simulation workflow, from simulation configuration to trace collection and analysis. The ns-3 simulation core supports research on both IP and non-IP based networks. However, the large majority of its users focuses on wireless/IP simulations which involve models for Wireless Fidelity (Wi-Fi), Worldwide Interoperability for Microwave Access (WiMAX), or Long Term Evolution (LTE) for layers 1 and 2 and a variety of static or dynamic routing protocols.

The LTE implementation in ns-3 started within the LENA project, which implemented the LTE and Evolved Packet Core (EPC) network functionalities. The flexibility of the solution allow emulating different network topologies. Moreover, the modular design of ns-3 permits creating additional functionalities and test them in the tool.

With the $5 \mathrm{G}$ in the horizon, the NYU Wireless created a fork of the ns-3 LENA project and created a module to support transmissions in the mmWave bands. In the past few months, an important effort is being made to combine the mmWave module to the 5G LENA. The first version of the 5G LENA simulator was released in February 2019.

\subsubsection{The mmWave module}

The mmWave module for ns-3 can be used to simulate 5G cellular networks operating in the mmWave band. Figure 4.1 illustrates the network architecture of the mmWave module for ns-3. As it can be seen, the mmWave module is built on top of the LTE protocol stack, which means that it uses most of LTE 
network functionalities implemented in ns-3. The implementation details of the mmWave module are given in [104]. Some of the most important features of the last version of the mmWave module for ns-3 are listed below:

- Support of a wide range of channel models, including the 3GPP model for frequency spectrum above $6 \mathrm{GHz}$. The implementation details of the $3 \mathrm{GPP}$ channel model for frequencies larger than $6 \mathrm{GHz}$ can be found in [105].

- The channel model implementation allows using realistic wave propagation data sources such as ray tracing tools or traces from real measurement campaigns.

- Physical (PHY) and Medium Access Control (MAC) layer classes have been redesigned for simulating mmWave communication networks.

- Custom schedulers for the dynamic Time Division Duplexing (TDD) format.

- Possibility to perform Carrier Aggregation (CA) in the MAC layer.

- Dual Connectivity with LTE base stations, with fast secondary cell handover and channel tracking through the legacy network.

\subsection{Extension of the mmWave module}

This subsection details the necessary modifications introduced in the ns-3 mmWave module to support beam management within the framework of NR. Figure 4.2 illustrates the diagram of classes related to the contribution to ns-3 mmWave module. The source code of the simulator used to obtain the results of this Thesis can be downloaded from [106].

\subsubsection{Flexible radio frame}

The frame structure in the ns-3 mmWave module required modifications in order to accommodate the NR specifications. The common class object MmWavePhyMacCommon stores the customisable variables affecting the PHY and MAC layers. This class was extended to include an enumerator variable m_scs that determines the system Sub-Carrier Spacing (SCS). There can only be one possible value for the whole system. From this value, the simulator calculates in the MmWavePhyMacCommon: :SetScs method the remaining frame structure variables: m_symbolPeriod, m_symbolsPerSlot, m_symbolsPerSuframe and m_slotsPerSuframe 


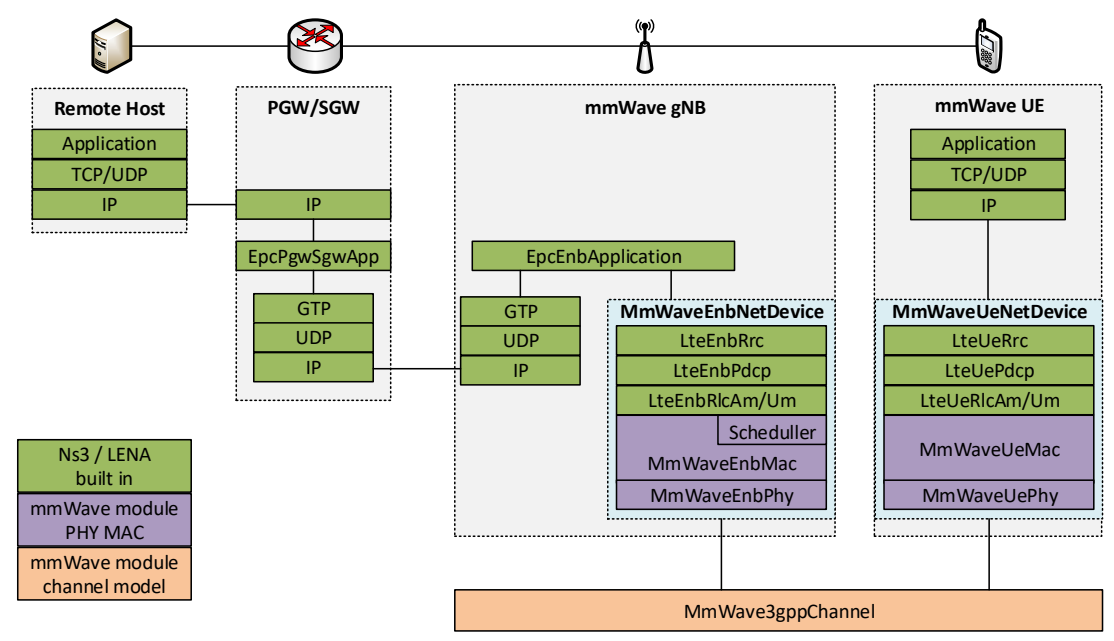

Figure 4.1: End-to-end ns-3 network architecture with the mmWave module over the LENA LTE protocol stack.

\section{Codebook}

The beam directions are ruled by a precoding matrix called codebook. Each instance of MmWaveBeamManagement has one codebook, which is loaded by calling MmWaveBeamManagement : : LoadCodebookFile method. The beam management implementation assumes that beamforming vectors are rows in the codebook matrix, and the beam id is directly mapped to the row index of the codebook matrix. Therefore, the number of beam indexes shall not exceed the number of rows in the codebook. Each row of the matrix has a certain number of complex values, which corresponds to the exact number of radiation elements in the antenna array. The codebook must be generated according to the design of the antenna array determined in AntennaArrayModel. The values of numAntennasH and numAntennasV in the MmWaveSpectrumPhy class determine whether the antenna array is rectangular, squared or linear. Other antenna geometries are not supported.

\subsubsection{Beam management}

A new MmWaveBeamManagement class is introduced in the mmWave module for ns-3. The NR base station (gNB) and User Equipment (UE) mmWave 


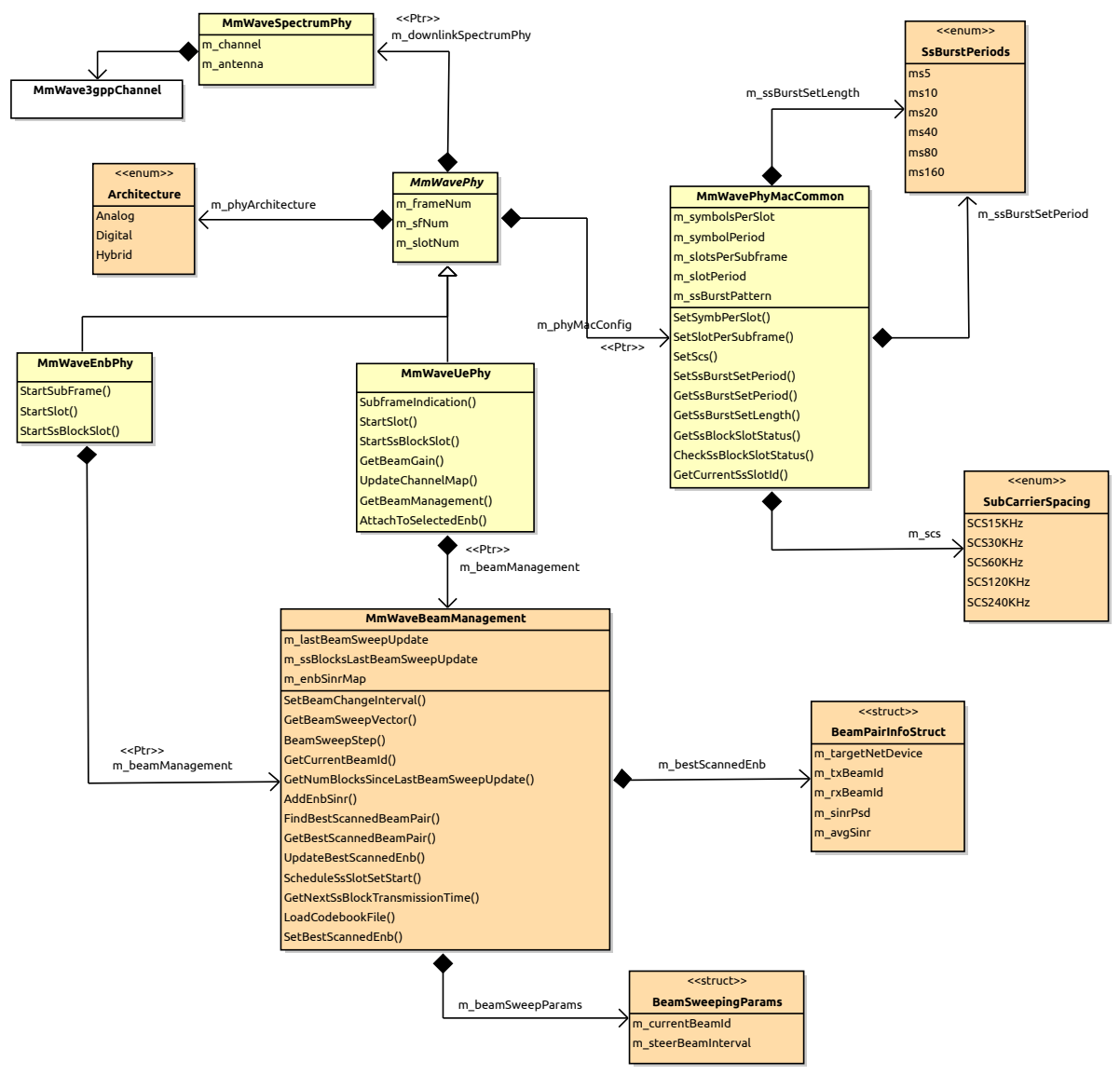

Figure 4.2: UML class diagram of the beam management framework for ns-3. New classes are coloured in orange, and the former ns-3 mmWave module classes that have been modified are coloured in yellow. 


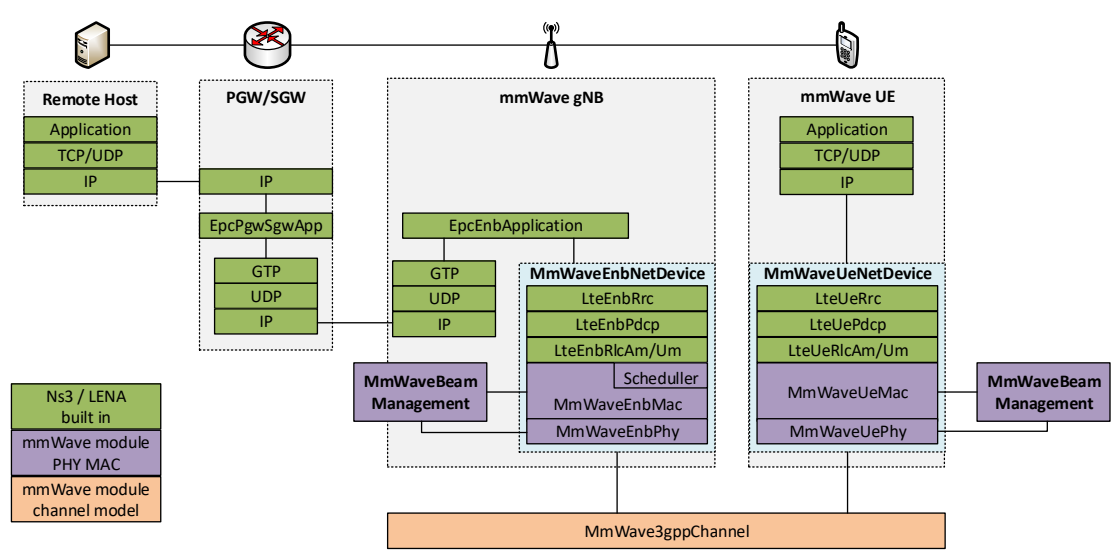

Figure 4.3: The devised NR-compliant beam management framework extension interacts with the mmWave module in different levels.

device classes instantiate independent MmWaveBeamManagement objects, which are also controlled by the MmWaveUePhy and MmWaveEnbPhy through the m_beamManagement class member in order to perform beam-related operations. Figure 4.3 illustrates the end-to-end network architecture in ns-3 with the beam management class. The MmWaveBeamManagement class manages the beamforming vectors and regulates the beam sweeping, beam measurement and beam determination procedures to find and maintain the best pair of beams for data transmission and reception.

The MmWaveBeamManagement class stores BeamPairInfoStruct objects for each managed beam. This BeamPairInfoStruct object contains a single transmit beam id m_txBeamId to a target receiver identified in $\mathrm{m}_{-}$targetNetDevice. The m_rxBeamId field contains the beam id used in reception. The BeamPairInfoStruct object also contains channel quality information in terms of Signal to Interference plus Noise Ratio (SINR): m_sinrPsd contains the Power Spectral Density (PSD) of each configured sub-band, which averaged value is stored in m_avgSinr. The choice of SINR is motivated by the fact that it is one of the measurement report metrics supported by $3 \mathrm{GPP}$ for NR and SINR can be easily mapped to other of the supported metrics in [90]. 


\section{Beam sweeping}

The initial access in Standalone (SA) mmWave NR deployments is based on beam sweeping, in which a base station sequentially uses the beams from its predefined codebook to transmit Synchronization Signal (SS)/Physical Broadcast Channel (PBCH)-Block (SSB) in the programmed time instants, covering the whole angular domain. UEs will try to detect the SSB on different directions by sequentially switching its beams extracted from its independent codebook. The MmWaveBeamManagement class has a BeamSweepingParams member that governs the beam sweeping procedure. This new class contains the id of the currently used beam (m_currentBeamId) and the beam change periodicity m_steerBeamInterval.

SSB transmission opportunities in the time domain are fixed and depends on the numerology. 3GPP determines the [86]. In MmWavePhyMacCommon, the m_ssBurstPattern class member stores the indexes of the starting Orthogonal Frequency Division Multiplexing (OFDM) symbol of the 4-symbol-long SSB referenced to the beginning of the current SSB transmission window or SS burst. This sort of SSB transmission pattern is initialized when setting the system numerology by calling MmWavePhyMacCommon: :SetScs. Assuming carrier frequencies over $6 \mathrm{GHz}$, the possible SCS values can be $120 \mathrm{kHz}$ or $240 \mathrm{kHz}$. In the current version of the simulator, only one numerology is supported. This limitation forces the SCS value to be $120 \mathrm{kHz}$, which is the only value compatible with data and control transmission in the mmWave regime.

SSB transmissions have a maximum length of $5 \mathrm{~ms}$, which is the minimum periodicity of the SSB burst [86]. It is possible to configure the SSB transmission window periodicity in the m_ssBurstSetPeriod in the MmWavePhyMacCommon class, whose possible values can be $5 \mathrm{~ms}, 10 \mathrm{~ms}, 20 \mathrm{~ms}$, $40 \mathrm{~ms}, 80 \mathrm{~ms}$ or $160 \mathrm{~ms}$. For initial cell selection, a UE may assume an SS burst periodicity of $20 \mathrm{~ms}$ [86], but this is not an stringent requirement and the network can choose another standardised period.

For carrier frequencies larger than $6 \mathrm{GHz}$, the maximum number of SSB transmission within the $5 \mathrm{~ms}-$ long SS burst is 64 . However, the number of SSB transmissions is configurable with the m_maxSsBlockSlotId variable. The m_currentSsBlockSlotId keeps track of the number of transmitted SSB index in the current SS burst.

The implementation of the beam sweeping in the mmWave module for ns-3 is illustrated in Figure 4.4. The gNB transmits each SSB on a single beam, and the beams used by the gNB are sequentially changed from one SSB transmission to the next one. In reception, UEs listen from the beams indicated at the beginning of the SS burst. The gNB also determines the number of resources to allocate for downlink signaling, including SSBs, in the subframe and the 
time location of the SSBs from m_ssBurstPattern. This resource allocation is implemented in MmWavePhyMacCommon: :GetDlBlockSize method at MAC layer.

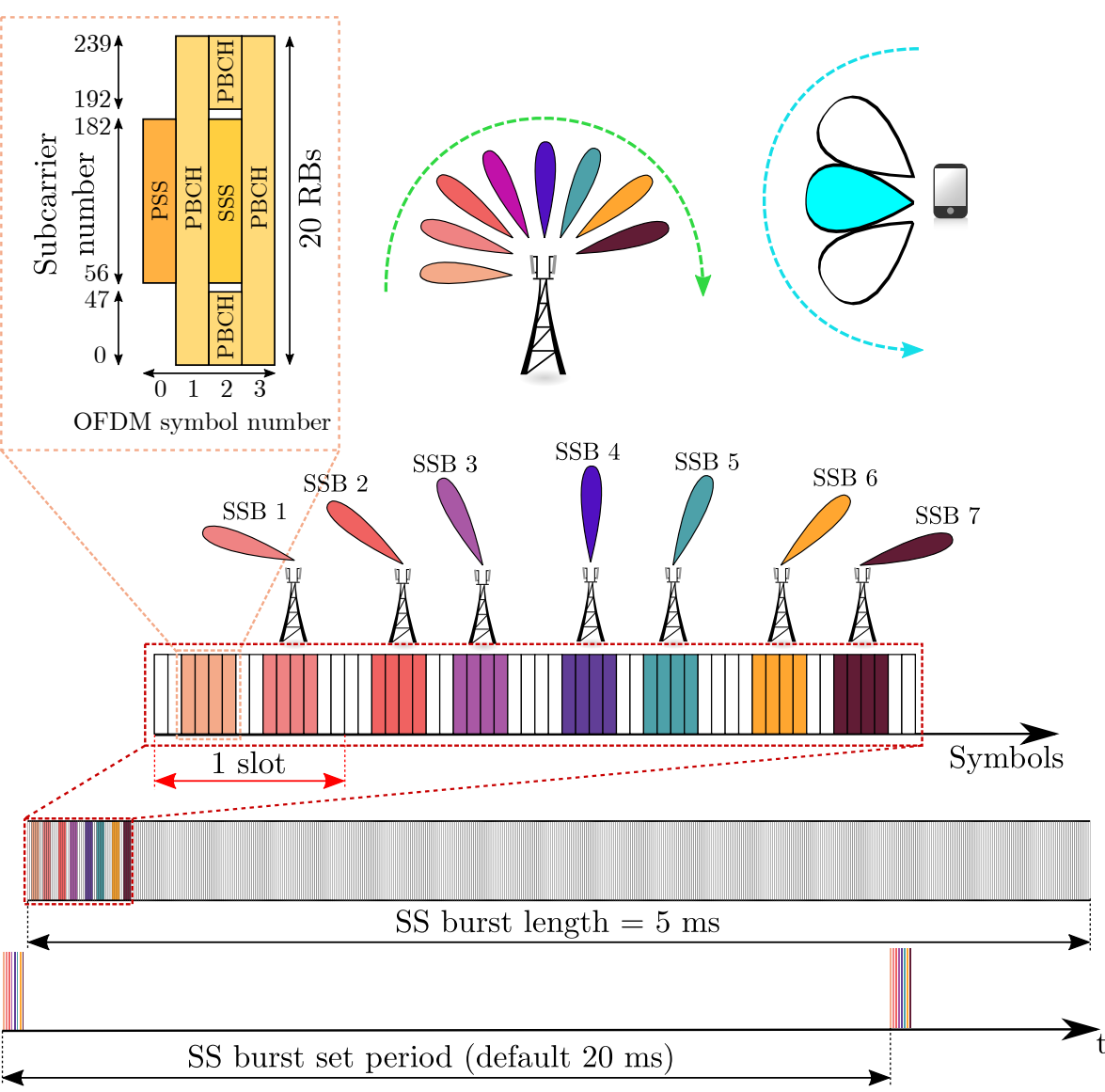

Figure 4.4: The beam sweeping procedure implemented in ns-3 mmWave module. In the example, $N=8$ different beams are used to transmit SSB. The UE measures the received signals from a fixed UE beam during the whole SSB window. 


\section{Beam measurement}

During the beam sweeping process, the UE measures the received SINR of all the beam combinations and stores the measurements in to a map as described below. The selection of SINR as the beam quality indicator is contemplated by the NR standard [90] and the SINR calculation was the only supported choice by the ns- 3 mmWavemodule at the time of writing this Thesis.

In the mmWave module of ns-3, the classes MmWaveEnbPhy and MmWaveUePhy of the PHY layer simulate the beginning and end of the frames, subframes and slots and perform the channel measurements [107]. In the implementation for this Thesis, beam measurements run in a thread in parallel to the regular data transmission in slots. On the one hand, the class MmWaveEnbPhy: :StartSsBlockSlot the gNB changes the beam used to transmit SSB each time a new block is to be transmitted. On the other hand, MmWaveUePhy: :StartSsBlockSlot measures the signal level from its current receiving beam. The MmWavePhy obtains the signal power level from the MmWaveSpectrumPhy class.

On the other hand, the UE may additionally measure and report beams with Channel State Information Reference Signals (CSI-RS) resources. CSI-RS beam measurements and reports are performed if the MmWaveBeamManagement::m_beamReportingEnabled flag is set to true. The beam management implementation for this Thesis only supports periodic CSI-RS reporting, and the reporting period is configured in MmWaveBeamManagement: :m_beamReportingPeriod. Note that the supported CSI-RS period values are $10 \mathrm{~ms}, 20 \mathrm{~ms}, 40 \mathrm{~ms}$ and $80 \mathrm{~ms}$ as detailed in Section 2.1.3.

Beam measurements of CSI-RS resources are ruled with the class MmWaveEnbPhy: :AcquaringPeriodicCsiValuesRoutine and run in an independent thread in parallel to the normal slot functionality. This routine consults the MmWaveBeamManagement in order to obtain the list of beam pairs to monitor with CSI-RS resources. The manager returns a data structure called BeamTrackingParams with the list of beam pairs and the reporting period. The MmWaveEnbPhy perfoms the measurements in the indicated time and reports the measured beam SINR to the gNB, which updates channel information.

Both analog and digital beamforming architectures are implemented at the PHY layer. The m_phyArchitecture member in the MmWavePhy class determine whether the layer uses analog or digital beamforming. Only one architecture can be used in the simulated deployment. Independently of the selection on the beamforming architecture, MmWaveUePhy and MmWaveEnbPhy use their codebooks to determine the best direction. The difference between analog or digital architectures implies that UEs with digital transceivers are able to detect 


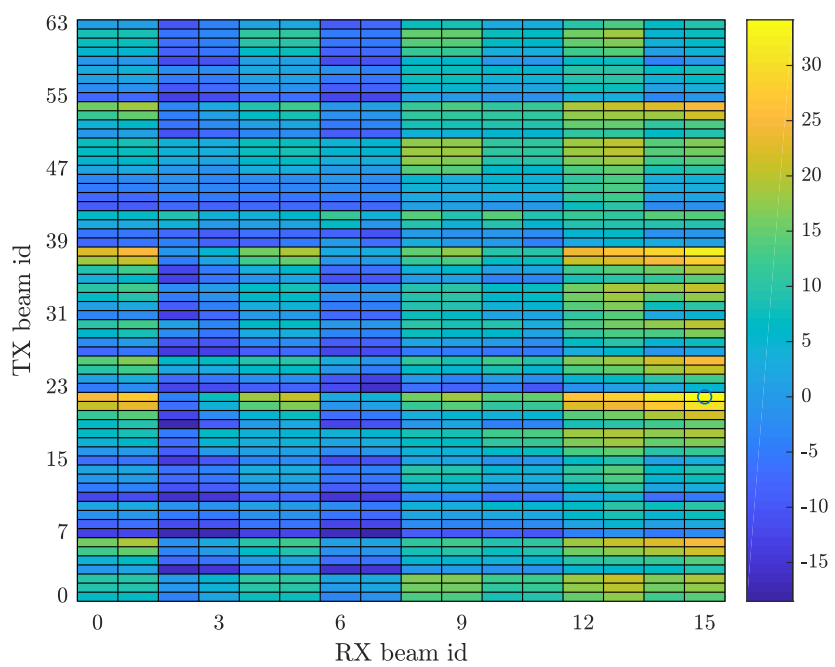

Figure 4.5: A realisation of the beam pair map containing the reported SINR by a given UE.

SSBs from all the receiving beams at the same time, while analog transceivers can only detect the synchronisation signal from a single receiving beam at a time. Finally, the UE stores the measurements in the m_enbSinrMap in the MmWaveBeamManagement.

A possible SINR map diagram is depicted in Figure 4.5. This map relates the detected gNB and another map relating all the combinations of transmit (gNB) and receive (UE) beams and, for each pair, the sub-band SINR and averaged values.

\section{Beam determination}

Once the UE completes a beam sweeping cycle and the UE has fresh SINR measurements from all the transmit and receive beams, then the MmWaveBeamManagement class selects the pair of beams with the highest SINR level. This procedure is referred as beam determination. Serving as a visual example of this procedure, Figure 4.5 illustrates a colour map of the grid of measured SINR values extracted from m_enbSinrMap. The vertical axis represents the transmit (TX) or gNB beam ids and the horizontal axis the receive (RX) or UE beam ids. This example assumed rectangular arrays in both gNB and UE sides. The gNB is configured with $16 \times 4$ antennas elements and 64 beams, whilst the UE is equipped with $8 \times 2$ antennas and 16 beams. The beam 
determination algorithm selects the pair of transmit and receive beams that reported the largest SINR level. In the example snapshot in Figure 4.5, the selected beam pair (circled slot) corrensponds to the transmit beam id 22 and the receive beam id 15 .

The decision on the best pair of beams, or optimum beam pair, is ideally notified to the target gNB to the peer MmWaveBeamManagement class. In case of using analog architectures, the beam update will happen when the SSB is transmitted on the best transmit beam, which means this update takes multiple SSBs in order to scan all directions. But in case digital architectures are deployed, the beam update happens at the same instant time each SSB is received.

For analog beamforming architecture, completing a whole beam sweeping cycle might be slow. This is why, eventually, the UE could not wait to complete the SINR map and update the pair of beams used for transmission and reception through the next pair of beams that provide a sufficiently good SINR level.

\section{Initial access and start of beam tracking}

Once the beam pair for data transmission is determined, UE triggers an ideal collision-free random access using the appropriate beams to connect with the best serving cell. In analog beamforming architectures, Random Access Channel (RACH) occasions are synchronised with the SSB transmission. This means that the $\mathrm{RACH}$ procedure is initiated on the optimal gNB beam at the same time the gNB transmits the SSB on the same beam. In fully-digital transceivers, $\mathrm{RACH}$ occassions do not wait for the SSB transmission since the gNB is able to detect signals from all its receiving beams at the same time. After the gNB and UE are connected, the beam manager at the UE keeps updating its internal channel condition map for beam tracking and link adaptation.

In the beam management framework implemented for this Thesis, beam tracking updates are based on beam sweeping SSB and CSI-RS measurements, which is in alignment with the NR standards [87]: the 5G network may instruct UEs to report beam quality metrics from measuring CSI-RS, SSBs or both signals. If CSI-RS measurement is enabled, beam updates are more frequent, which can result in a better beam tracking.

If CSI-RS measurements are enabled, beam tracking is performed differently to the SSB-only beam tracking. The gNB instructs the UE to perform a number of beam measurements on specific beams at a specific time instants. Once the beam measurements are completed, the UE reports the experienced SINR level of each beam pair combination. Then, the gNB updates the corresponding entries of its internal m_enbSinrMap and, based on the updated 
channel information, provisions the UE with the list of beams to monitor with a new BeamTrackingParams.

\subsection{Beam management framework validation}

As a proof-of-concept of the beam management framework introduced in this work, a simple urban scenario was set up consisting on a single gNB and a UE moving at constant speed, with acceleration set to zero. The gNB was located at the centre of the scenario, which is the origin of the coordinates system $(0,0)$. For this exercise, the Urban Macro-cell (UMa) scenario defined in the 3GPP channel model for frequencies above $6 \mathrm{GHz}$ [5] was selected.

It is assumed that the gNB mounts a single rectangular antenna array with 64 radiation elements, 16 omnidirectional radiation elements in the horizontal plane and 4 in the vertical plane. The UE is also equipped with one rectangular antenna array with 16 omnidirectional radiation elements, 8 in the horizontal plane and 2 in the vertical plane. Both gNB and UE perform 3D codebookbased beamforming, with as many beams per plane as the number of radiation elements per plane. The gNB and UE codebooks were generated as defined in Section 3.3.

The rest of the simulation parameters are summarised in Table 4.1.

\subsubsection{Circular walk}

In this first example, the objective is to demonstrate that the pair of beams are changed as the UE moves and the optimal beams to connect with the gNB changes. For that aim, a circular walk around the base station was used to visualise the beam pair evolution through all the beams of the gNB. For the sake of simplicity of the beam pair evolution, the UE is in Line of Sight (LoS) condition during the simulation.

The circular walk implemented for this experiment takes the UE to complete a whole walk around the base station while maintaining a constant distance $r$ to the gNB. Figure 4.6 illustrates how the circular walk works. The UE starting position is on the $x$-axis and the walk does not finish until the UE arrives at the same starting position.

This first analysis is interested in illustrating how beams changes along the circular walk around the gNB with different radii, including 20, 40, 60 and 100 metres. Figure 4.7 illustrates the transmit and receive combination of beams used throughout the whole circular walk of radii 20 and 40 metres, and Figure 4.8 represents the same beam pair evolution in the two furthest distances being considered: 60 and 100 metres. In both figures there is a clear 
Table 4.1: Beam management proof-of-concept simulation parameters.

\begin{tabular}{|c|c|}
\hline Simulation parameter & Value \\
\hline Channel & 3GPP Urban Macro (UMa) \\
\hline Carrier frequency & $28 \mathrm{GHz}$ \\
\hline System bandwidth & $400 \mathrm{MHz}$ \\
\hline Transmit Power & $23 \mathrm{dBm}$ \\
\hline gNB height & $25 \mathrm{~m}$ \\
\hline gNB antenna elements & 64 (16 horizontal and 4 vertical) \\
\hline gNB codebook & 3D Kronecker codebook \\
\hline gNB beams & 64 (16 horizontal and 4 vertical) \\
\hline Sectorisation & Omnidirectional \\
\hline UE height & $1.5 \mathrm{~m}$ \\
\hline UE speed & $1 \mathrm{~m} / \mathrm{s}$ \\
\hline UE antenna elements & 16 ( 8 horizontal and 2 vertical $)$ \\
\hline UE codebook & 3D Kronecker codebook \\
\hline UE beams & 16 (8 horizontal and 2 vertical $)$ \\
\hline SS burst period & $20 \mathrm{~ms}$ \\
\hline TCP congestion window protocol & Cubic [108] \\
\hline Simultaneous TCP flows & 4 \\
\hline Simulation duration & $10 \mathrm{~s}$ \\
\hline Number of independent runs & 10 \\
\hline
\end{tabular}




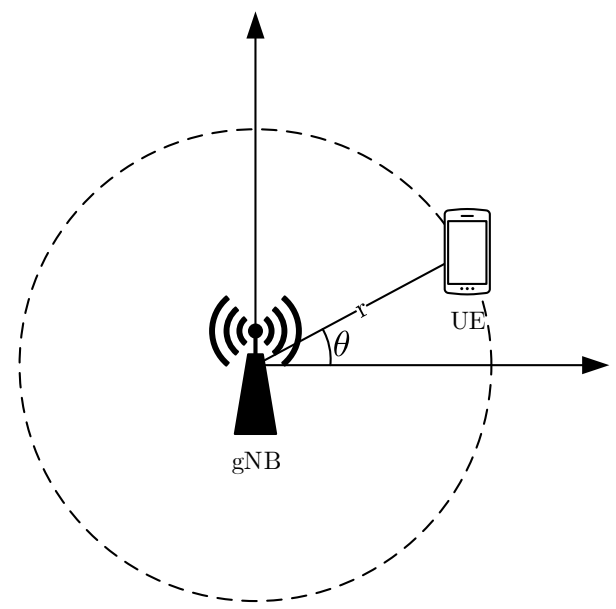

Figure 4.6: In the circular walk, the gNB is at the centre of the scenario and the UE walks around the gNB, always maintaining the same physical separation.

symmetry of the beam evolution in $\theta=0$ rad. This is reasonable since the antenna arrays are contained on a vertical plane and no sectorised antennas are being used. As a result, both sides of the antenna array are equivalent.

Comparing the results in Figure 4.7, the gNB beam are all different between the two radii of 20 metres and 40 metres. The only reason is the change on the elevation beam. Note that beam ids are ordered sequentially in the same elevation beam. If the number of azimuth beams of a given array is $N_{\text {azimuth }}$, beam id zero adjacent beam ids in the same elevation plane are 1 and $N_{\text {azimuth }}-1$. Adjacent beams in the same azimuth plane are separated by $N_{\text {azimuth }}$ beam ids.

UE beam id are different in the two walks in Figure 4.7. The UE uses 5 different beams when is 20 metres away from the gNB, whereas the number of different beam ids is 7 when the distance is 40 metres away. The reason is that beam prints are not homogeneous in elevation as illustrated in Figure 3.6. Note that the elevation angle at 20 metres distance to the gNB is about $40^{\circ}$.

UE beams in Figure 4.8 are completely different to the UE beams in Figure 4.7 due to the change of elevation beam on the UE side in this case. Also note that with a distance of 100 metres to the base station, the gNB beams also change their elevation beam. 

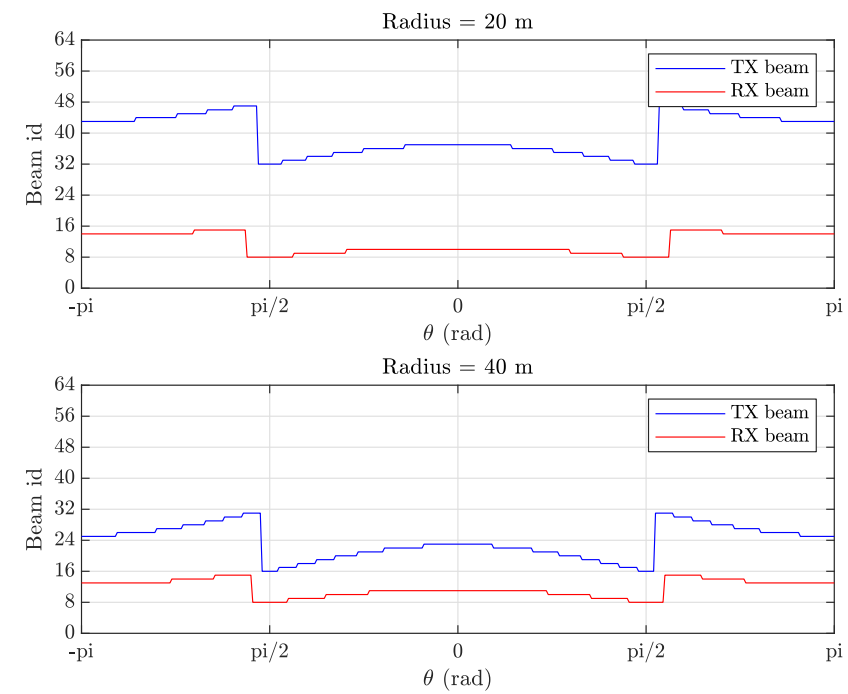

Figure 4.7: Circular walks with distances to the gNB of 30 and 40 metres.
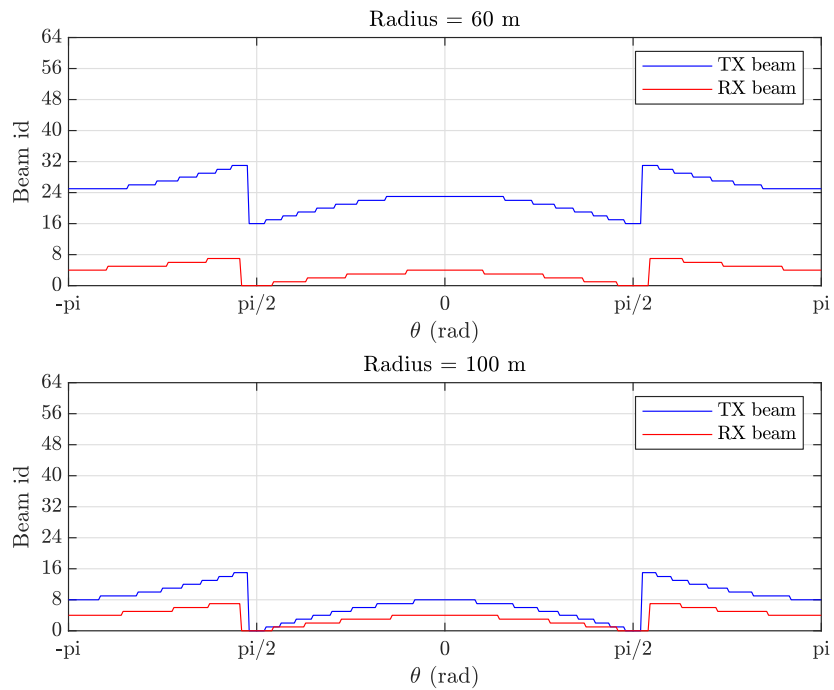

Figure 4.8: Circular walks with distances to the gNB of 60 and 80 metres. 


\subsubsection{Linear walk}

The objective of this second example is to check the effect of blockages in the TCP performance. Figure 4.9 illustrates the new scenario set for this second exercise. As it can be seen, the UE moves along the $x$-axis. This time, the linear movement is done at a constant speed of $15 \mathrm{~m} / \mathrm{s}$, which is a typical vehicular speed in urban scenarios. The UE stars its trip at point $(-80,50)$ and finishes it at point $(70,-50)$. In total, the trip lasts for 10 seconds at the vehicular seed in consideration.

A vehicular speed was selected in order to force beam changes more frequently than at pedestrian speeds. Figure 4.9 also illustrates the UE and gNB beams used in the whole ride. The SS burst periodicity was set to the longest possible value: $160 \mathrm{~ms}$. The motivation of selecting such large periodicity is to check the influence of large beam updates in the experienced throughput.

At the beginning of the ride, the gNB switches the elevation beam, from beam 4 to beam 20. The change of elevation beam is clear given that the gNB beams belonging the same horizontal plane are grouped in 16 beams, from 0 to 15, and a given beam in a given direction in azimuth. The following
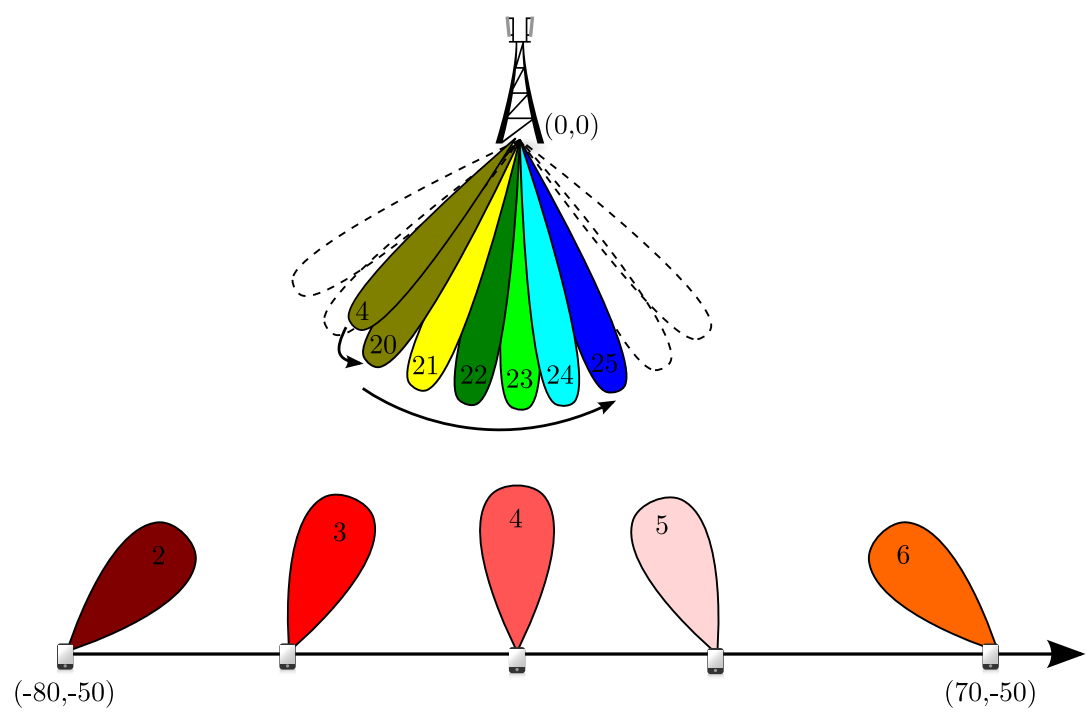

Figure 4.9: To track the mobility of the UE, the gNB first switches the elevation beam (same colour, beam 4 to beam 20) and then it scans beams in the same horizontal codebook (different colours). On the UE side, the used beams belong to the same horizontal codebook. 
beam changes are done in the same horizontal codebook since the numbers corresponding to the beam identifiers are consecutive. On the UE side, beam changes occur within the same horizontal codebook.

The UE generates TCP traffic and consumes all the system resources, i.e., full buffer. The TCP version is Cubic, which is largely adopted in modern communication networks.

Figure 4.10 and Figure 4.11 show the evolution of the throughput trends and beam pairs trends in the communication link. Even if analog beamforming is assumed in the system modelling, fully-digital beamforming architectures are considered for performance evaluation purposes. The digital performance is the best performance the system can achieve, so it is a good reference. In addition, two beam tracking strategies are assumed: one is based on beam sweeping SSB signals and the second one combines SSB with CSI-RS in a given measurement reporting configuration generating measurements every $5 \mathrm{~ms}$. Note that the beam tracking in digital is done with SSB measurements only.

For digital and the two analog beam tracking configurations, Figure 4.10 shows the variations in LoS conditions and Figure 4.11 is the same walk in LoS path but with blockages, which are happened at $2 \mathrm{~s}, 3.5 \mathrm{~s}, 6 \mathrm{~s}$ and $7.5 \mathrm{~s}$. Periods in Non Line of Sight (NLoS) are greyed out in Figure 4.11 for a better understanding of the influence of blockages. Despite TCP traffic is full buffer, the throughput slow start during the first two seconds of the simulation is due to the selection of the Cubic TCP traffic profile and the fact that the congestion window opens up slowly [108]. ns-3 implements several TCP congestion algorithms. The selection of the Cubic algorithm was based on its suitability for high-rate connections [108].

As it can be seen in Figure 4.10, beam tracking with additional CSI-RS in LoS maintains the same optimal data rate achieved with the digital beamforming architecture. A different situation occurs with SSB-only beam tracking: the frequent beam changes due to the UE speed are not followed by this simple beam tracking strategy. It can be easily seen that the detection of the optimal beam pairs is delayed for several beam updates. As a consequence, the experienced throughput is reduced along this delay: only when the optimal pair of beams is found, then the data rate can recover until reaching the rate in the digital beamforming case if the beam alignment is maintained. As occurred in the beginning of the simulation, the data rate recovery is slow due to the TCP cubic congestion window.

The appearance of blockages in the walk influences the throughput experienced with all the three beam tracking configurations due to the signal degradation in NLoS. As it can be seen in Figure 4.11, both beam tracking strategies with analog beamforming fall under the digital beamforming figures. This is due to the intrinsic delay in determining the optimum pair of beams in the 

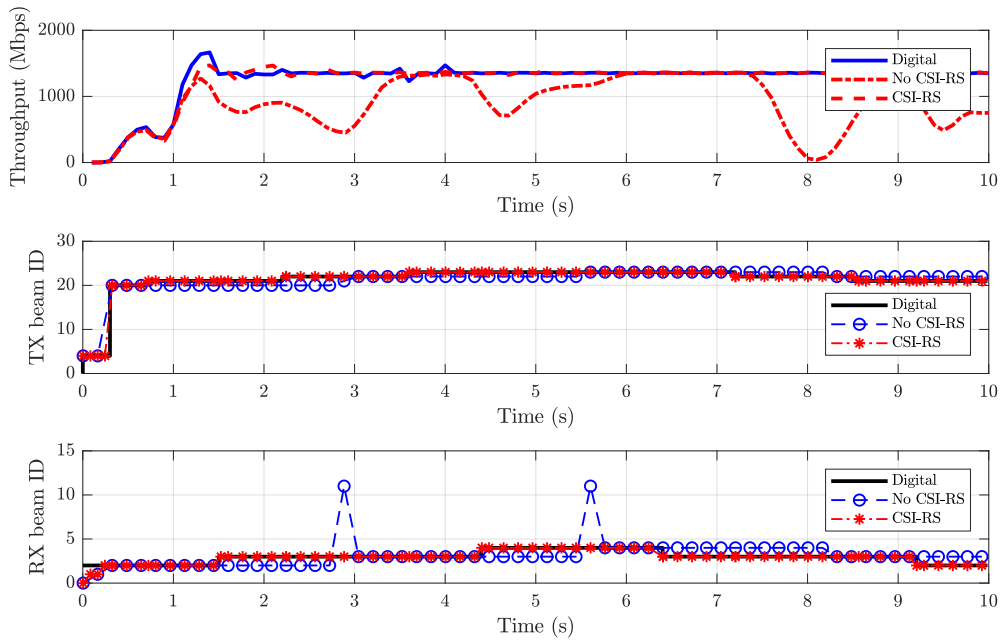

Figure 4.10: Linear walk in LoS.
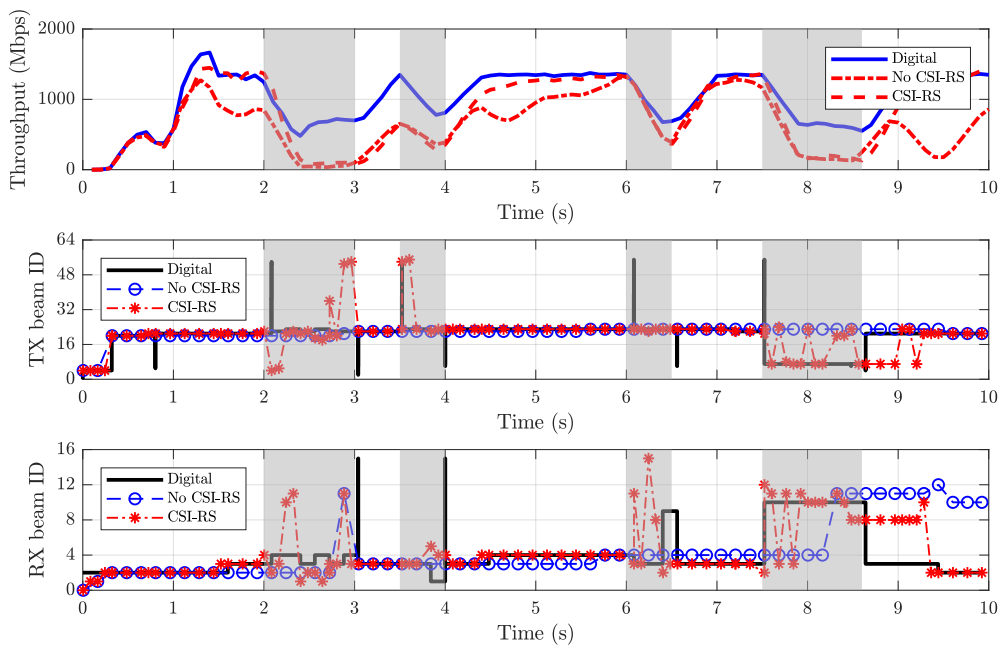

Figure 4.11: Linear walk with some LoS to NLoS transitions and viceversa due to the appearance of blockages between the gNB and UE. 
analog beamforming architecture. In some periods like seconds 2.5 to 3 , the throughput experienced by the two analog strategies suffer a complete service disruption and no data is transmitted.

As it can be seen in 4.11, and only considering the NLoS time areas, both analog beam tracking strategies consider beams which are far from the optimal ones for some moments. It can be said that the analog beam tracking is poor. This time, CSI-RS measurement reporting slightly accelerates the search of the optimal pair of beams; only in seconds 4 to 6 the benefit of monitoring CSI-RS has a significant improvement over not doing it.

The digital beam mask in the NLoS regions in 4.11 presents some strong peaks which are difficult to understand. In order to create efficient beam tracking strategies, it is necessary to have a full understanding of the channel propagation conditions. Unfortunately, the 3GPP channel model is stochastic and beam powers and directions are randomly generated. Therefore, other channel models based on a realistic characterisation of the propagation conditions must be considered.

\subsection{Visualisation}

Since $5 \mathrm{G}$ will not only be a mere evolution of existing cellular standards, but will extend cellular communications to a wide range of novel applications and use cases involving vertical industries and other novel stakeholders, it will be especially important that the benefit of the solutions and frame-works developed in $5 \mathrm{G}$ is well-demonstrated to a wide audience. Note that, in parallel to the research and standardisation work, the policy-makers and regulators are making decisions that will have a profound impact on the future deployment of this technology. For instance, they have to decide on the availability of more frequency spectrum in the near future for mobile communications, or on the kind of wireless technology to be promoted for the communications between autonomous cars in Europe.

To help the work and decisions of the above-mentioned actors (standardisation organisations, researchers, policy-makers, etc.), it is necessary to provide them meaningful insights about the technology, and one extremely useful way to do that is through the visualisation of the technology operation and performance. In fact, the METIS-II evaluation and visualisation approach is summarised in the sentence "one picture is worth a thousand words" [30].

$5 \mathrm{G}$ is a complex technology, with many different aspects to visualise whose number increases as the technology evolves, and with many different contributing entities. Therefore, the $5 \mathrm{G}$ community would obtain a great benefit from 
the availability of an open-source fully-customisable visualisation platform in which new concepts could be implemented easily and inexpensively.

Based on these considerations, METIS-II project decided to develop a 5G visualisation platform. Their objective was to design a full-fledged Three Dimension (3D) game-based platform with high user interactivity and high user immersion, easily customisable to show different aspects and concepts of the technology, and open to third parties [109]. The particular examples are detailed in Annex A.

\subsection{Conclusions}

This chapter of the Thesis has presented the simulation framework which will serve to test the beam tracking mechanisms in the later sections of the Thesis.

The selection of the ns-3 end-to-end network simulation is right given the long tradition in simulating different kinds of wireless networks. The mmWave module for ns-3 provides a solid foundation on which start creating the necessary simulation conditions to support the beam management framework in 3GPP NR. The results shown in this Chapter validates the beam management framework, since the network can switch beams according to the quality of the pair of beams being reported by the UE, achieving stabilised throughput under some scenario configurations.

The simple simulation scenarios set at the end of the present chapter validates the beam management framework. The beam management implementation has proved to properly track the channel conditions of the possible combinations of gNB and UE beams, and based of the measurement reports sent by the UE, the gNB is able to re-configure the beams on which data transmission is conveyed. The beam pair evolution in the circular walks obtained during simulation are in alignment with the codebook and antenna array combined radiation diagram. In the second exercise, the tracking ability on LoS and NLoS conditions are evaluated. Results form the second exercise showed that different beam tracking configurations provide different performance results. These results also stressed the necessity of creating good beam tracking strategies. And to that aim, a good understanding of the multipath propagation is needed and, hence, realistic channel models must be used.

From the collaboration in the METIS-II project, the information generated by means of computer simulators can be represented in a visualisation platform. The customisable METIS-II visualisation platform allows creating a particular story to narrate the novelties in the $5 \mathrm{G}$ technology. This effort can help to reach to a wider audience and ease the explanation of complex concepts. Moreover, 
the visualisation platform allows developing extensions such the ray tracing channel model introduced in Section 3.2.2. 



\section{Chapter 5}

\section{Beam Tracking Techniques}

This chapter of the Thesis presents the beam tracking strategies which are solely based on the Channel State Information (CSI) obtained from measuring and reporting the quality level of a given combination of Base Station (BS) and User Equipment (UE) beams. The beam tracking algorithms discussed in this chapter are in alignment with the Third Generation Partnership Project (3GPP) New Radio (NR) Release 16 specification. For that reason, beam information is obtained from the use of standard NR signals. Specifically, this Thesis work is focused on the use of downlink signals to estimate the channel, naming Synchronization Signal (SS)/Physical Broadcast Channel (PBCH)-Block (SSB) and Channel State Information Reference Signals (CSI-RS).

The beam tracking techniques detailed in this chapter are evaluated in two different urban scenarios. The first scenario in consideration is an Urban Macrocell (UMa) scenario with a single NR base station (gNB) with one antenna panel over the rooftop of a building. The second scenario is an Urban Microcell (UMi) scenario, where a single antenna panel is placed on the street level, a few meters over the user. In both scenarios, a single user moves along the scenario at a given speed.

This chapter is structured as follows:

- Section 5.1 details the simulation setup. In the first place, this section introduces the two urban scenarios and the network and system assumptions made. Secondly, the section defines the performance metrics used in this Thesis to evaluate the tracking efficiency of the beam tracking solutions proposed. Finally, this section introduces the baseline case, which is used as a reference to compare the performance of beam tracking algorithms with. 


\section{CHAPTER 5. BEAM TRACKING TECHNIQUES}

- Section 5.2 focuses on performing beam tracking with SSB signals only. Different mobility configurations and SSB periodicities are evaluated in real urban scenarios to determine the feasibility of beam tracking with SSB only.

- Section 5.3 complements the SSB-based beam tracking solution with the measurement of beamformed CSI-RS resources. This section covers the problem of creating the list of candidate beams to monitor and presents a solution based on selected beam measurements.

- Section 5.4 provides some insights on the dependence of the orientation of user devices in the coverage area of each beam. This orientation dependence is later validated by means of system-level simulations.

- Section 5.5 presents the conclusions drawn from the evaluation of the beam tracking strategies contained in the present chapter.

\subsection{Simulation setup}

\subsubsection{Simulated scenario}

The beam tracking strategies introduced in this chapter will be evaluated in very realistic urban scenarios. For this reason, the assessment will be performed with the channel model detailed in Chapter 3.2.2. The consideration of urban scenarios in this Thesis is due to their large probability of blockages and viability of communication through reflected rays. The intermittent availability of communication paths in Line of Sight $(\mathrm{LoS})$ requires finding alternative beams really fast, which makes beam tracking specially challenging and capital in Millimeter Wave (mmWave) communications.

The focus of the Thesis is on beam tracking users in mobility. Therefore, the network deployment consists on one gNB with a single multi-antenna panel and one moving UE. The UE mobility pattern is defined inside the ray-tracing tool in order to calculate the channel response at the different UE locations. The channel resolution is 0.1 meters. The UE moves linearly through a defined number of reference points. This enables modelling different mobility situations like, for instance, turning corners or moving side-to-side on a street.

Both gNB and UE are equipped with a Uniform Rectangular Array (URA). The gNB panel consists of 64 antenna elements, which are distributed in 16 columns and 4 rows. The antenna array at the UE has 16 antenna elements with 8 rows and 2 columns. The antenna arrays are contained in the $x y$ plane, and both arrays point towards the geographical north, which corresponds to the $z$-axis. In both arrays, the antenna element separation is half the wavelength. 
Both gNB and UE beams are extracted from their respective Kronecker codebooks as defined in Section 3.3. The number of beams in the azimuth plane matches the number of columns of the antenna panel, while the number of beams in elevation matches the number of rows. Therefore, the gNB has 16 beams in azimuth and 4 beams in elevation, whilst the UE has 8 beams in azimuth and 2 in elevation. In both codebooks, for the sake of simplicity, the downtilt parameter, $\beta$, is set to 1 .

It is assumed an Sub-Carrier Spacing (SCS) value of $120 \mathrm{kHz}$ for being one of the two possibilities for central frequencies in the mmWave band and being the only common option for control and data plane. In Frequency Range (FR) 2 (FR2), the maximum number of SSB transmissions within an SS burst $L$ is 64. Therefore, the $64 \mathrm{gNB}$ beams can fit in one transmission window, so the whole space is beam swept.

The virtual scenario used is the Manhattan model described in Section 3.2.2. Two different mmWave network deployments, UMa and UMi will be evaluated. The common simulation parameters are summarised in Table 5.1

There is a TCP application running at the UE continuously generating downlink data packets which makes use of all the available time/frequency resources. As discussed in Section 4.3, TCP Cubic has a slow opening window and it takes some time for the network to reach and stabilise the peak throughput served to a given UE. For this reason, all ns-3 simulations start with a transition time to the stationary (peak) throughput regime of 3 seconds. In this initial period, the UE is static in $\operatorname{LoS}$ to achieve the maximum throughput. After these initial 3 seconds, the UE starts moving at the specified speed.

The fundamental difference between UMa and UMi scenarios is the antenna height of the gNB. The height of the gNB antenna array has an important impact on the shape of the beam prints. Figure 5.1 illustrates the azimuth beam prints in a 200 metres long square area with the gNB antenna array in the centre of the square. Since the antenna panel is pointing to $z$ direction, there is beam symmetry with respect to the $x$-axis. Note that beam prints are generated on a plane at UE height level, not at ground level. Beam print areas are determined by hyperbolas, and not all the beam prints cover the same area. Take as an example the beam id 8 , which corresponds to the areas with the greenest colour. Beam print presence in the evaluation square is smaller in UMa beam prints in Figure 5.1(a) than in UMi in Figure 5.1(b)

The previous exercise is repeated for the elevation beam prints in Figure 5.2. As it can be seen in Figure 5.2(b), the elevation beam id 3 in UMi is confined in a small circular area of radius 14 metres around the base station, which is even a smaller area than beam id 2 in UMa in Figure 5.2(a). In the UMi scenario, the elevation beam id is likely to be beam id 0 , whereas in the UMa scenario the most likely beams are beam ids 0 or 3 . 


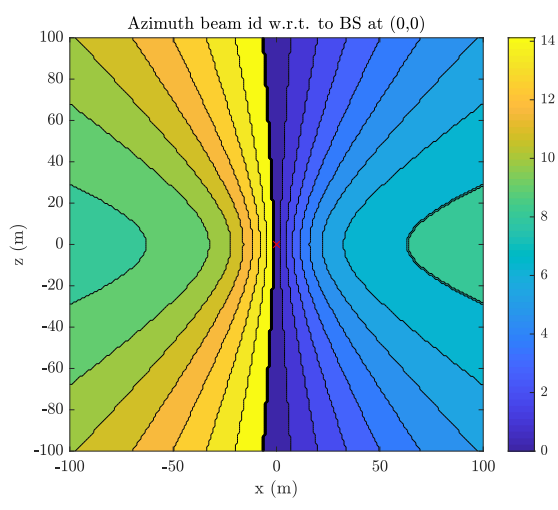

(a) UMa

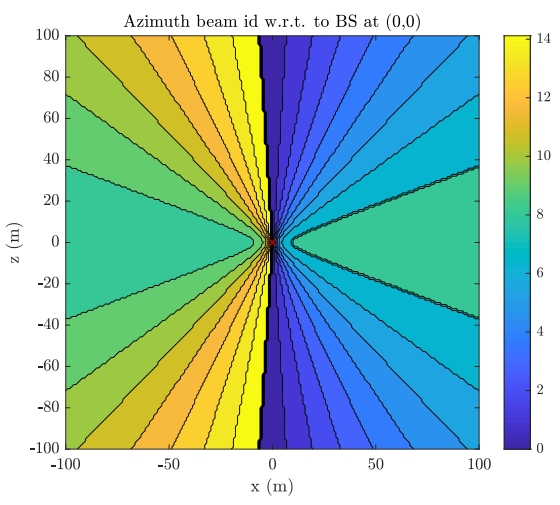

(b) UMi

Figure 5.1: Azimuth beam prints for UMa and UMi scenarios.

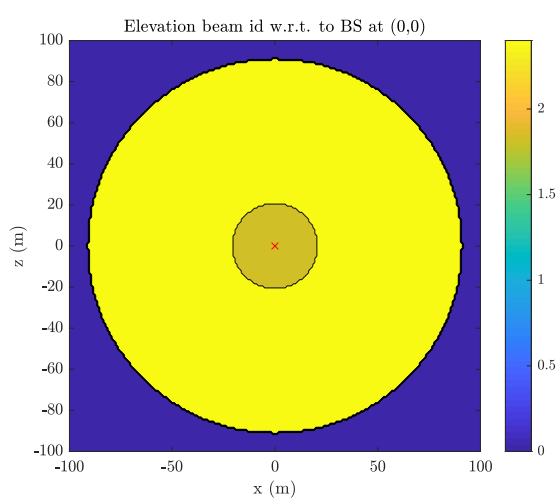

(a) UMa

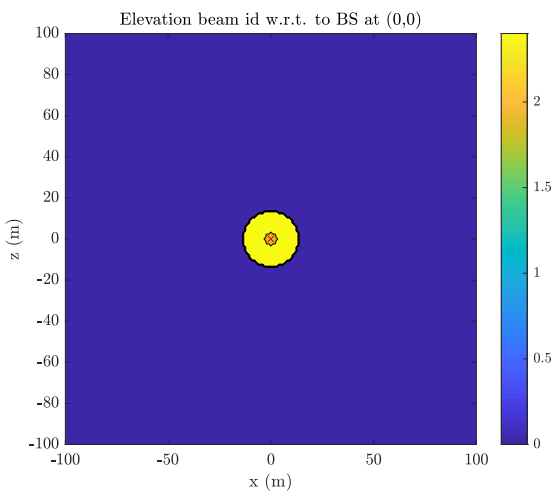

(b) UMi

Figure 5.2: Elevation beam prints for UMa and UMi scenarios. 
Table 5.1: Simulation parameters

\begin{tabular}{|c|c|}
\hline Parameter & Value \\
\hline Frequency & $28 \mathrm{GHz}$ \\
\hline Bandwidth & $400 \mathrm{MHz}$ \\
\hline SCS & $120 \mathrm{kHz}$ \\
\hline BS height & $20 \mathrm{~m}$ (UMa) / $5 \mathrm{~m}$ (UMi) \\
\hline TX power & $32 \mathrm{dBm}$ \\
\hline BS antenna elements & 64 (16 columns and 4 rows) \\
\hline BS codebook & 3D Kronecker codebook \\
\hline BS beams & 64 (16 horizontal and 4 vertical) \\
\hline UE height & $1.5 \mathrm{~m}$ \\
\hline Pedestrian speed & $1 \mathrm{~m} / \mathrm{s}$ \\
\hline Vehicular speed & $15 \mathrm{~m} / \mathrm{s}$ \\
\hline UE antenna elements & 16 ( 8 columns and 2 rows $)$ \\
\hline UE codebook & 3D Kronecker codebook \\
\hline UE beams & 16 ( 8 horizontal and 2 vertical) \\
\hline SS burst set period (ms) & $5,10,20,40,80$ or 160 \\
\hline CSI-RS resource period $(\mathrm{ms})$ & $0,10,20,40$ or 80 \\
\hline CSI-RS report period (ms) & $0,10,20,40$ or 80 \\
\hline Transmission Control Protocol (TCP) traffic & FTP downlink \\
\hline Network latency & $1 \mathrm{~ms}$ \\
\hline Packet size $(\mathrm{kB})$ & 14000 \\
\hline TCP congestion window & Cubic [108] \\
\hline Simultaneous TCP flows & 1 \\
\hline Stationary regime transition $t$ & $3 \mathrm{~s}$ \\
\hline
\end{tabular}

\section{Urban Macro-cell scenario}

In this setup, a single gNB with a single antenna array will be placed $25 \mathrm{~m}$ over the ground level. The BS is placed on the top of an existing building on the intersection of Bleeker St. and Mercer St. near the New York University campus in Downtown Manhattan in New York. Figure 5.3 shows a satellite image of the area of interest. In addition, the figure illustrates the location of the BS, as a red dot, and the user walk, as a red arrow. The UE walk considered is also illustrated in Figure 5.4 within the ray-tracing tool used in the Thesis.

The interest is on evaluating the beam tracking ability on situations with transitions from LoS to Non Line of Sight (NLoS). This requirement determined the UE trajectory considered. The UE starts its linear walk in Bleeker 


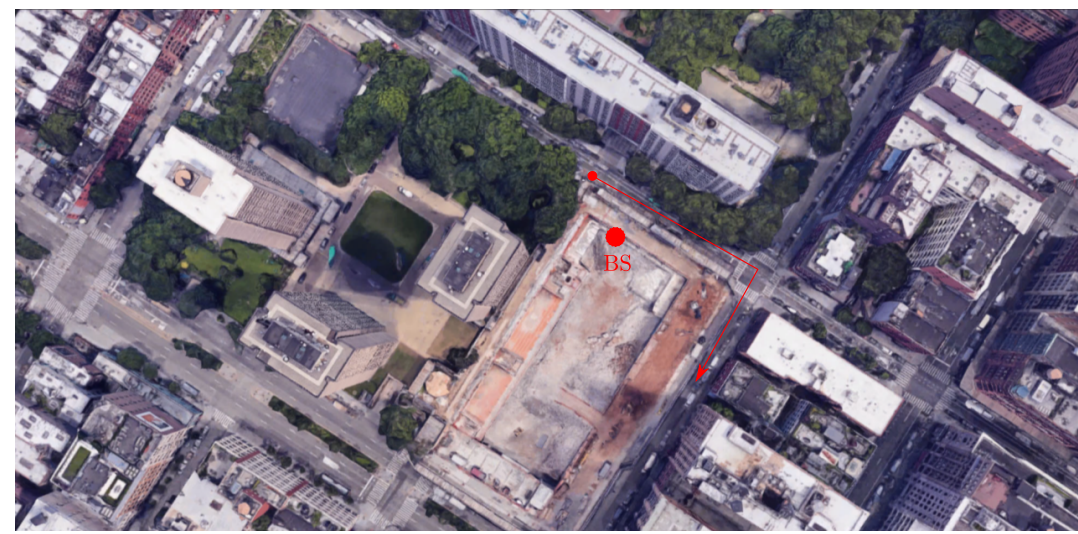

Figure 5.3: Satellite image from Google Maps of the zone considered in UMa. The location of the BS and the user walk path are visually represented by a red dot and a red arrow, respectively.

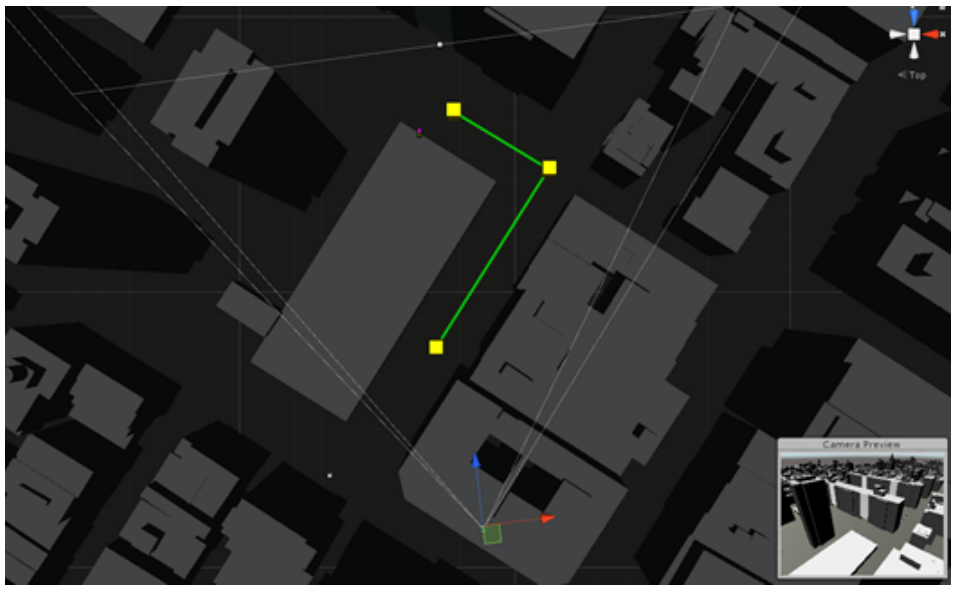

Figure 5.4: The UE walk path considered in UMa scenario. 
St. in LoS condition, and turns right at Mercer St. In this street the UE experiences an NLoS situation until the end of the walk since the building down the gNB antenna panel is blocking the direct signal path.

Figure 5.5 shows the optimal gNB beam prints in the region of interest for UMa. The beam print is a map of an area of interest which relates each location in the map with the beam id that provides the largest beam gain. A grid of points has been defined for the areas of interest with a spatial resolution of $10 \mathrm{~cm}$ and the optimal pair of beams has been calculated in each point for each considered rotation. When plotting the beam prints, each beam id has a different colour in order to ease the differentiation among beam ids.

Figure 5.5(a) illustrates the azimuth beam prints, whereas Figure 5.5(b) shows the elevation beam prints. Note that the optimal beam mask is created based on the beam index which provides the largest beam gain in the given location. The gNB antenna panel or BS is located, and represented by a red dot in Figure 5.5. Additionally, Figure 5.5 represents the UE walk in the scenario with a red arrow, indicating the walking direction.

\section{Urban Micro-cell scenario}

In the UMi scenario, the gNB antenna panel or BS is located at West 3rd St., between Thompson St. and Sullivan St. Figure 5.6 shows a satellite image of the area of interest and a visual representation of the location of the BS, as a red dot, and the user walk, as a red arrow.

The antenna height is set to 5 metres, simulating that it is placed on the building facade.

Figure 5.7 shows the optimal gNB beam prints in the region of interest for UMi. Figure 5.7(a) illustrates the azimuth beam prints, whereas Figure 5.7(b) shows the optimal elevation beam prints. As in the UMa case, the optimal beam mask is created based on the beam index which provides the largest beam gain in the given location.

Note that, in the first part of the route the beam prints are narrow and the path traverses many different optimal beams per unit of distance, while in the second part of the route the change of optimal beams is less frequent. Note also that due to reflection effects on the building where the antenna is located, an unexpected optimal azimuth beam print id 0 appears as a straight line on $z=839$ in Figure 5.7(a). As the distance to the gNB increases, the UE falls into different elevation gNB beam changes as illustrated in Figure 5.7(b). 


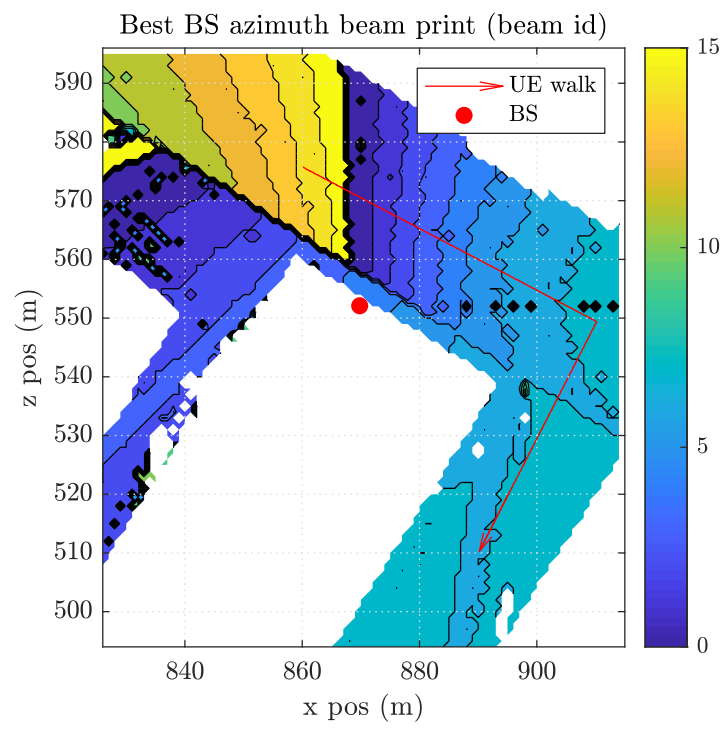

(a) Horizontal beams

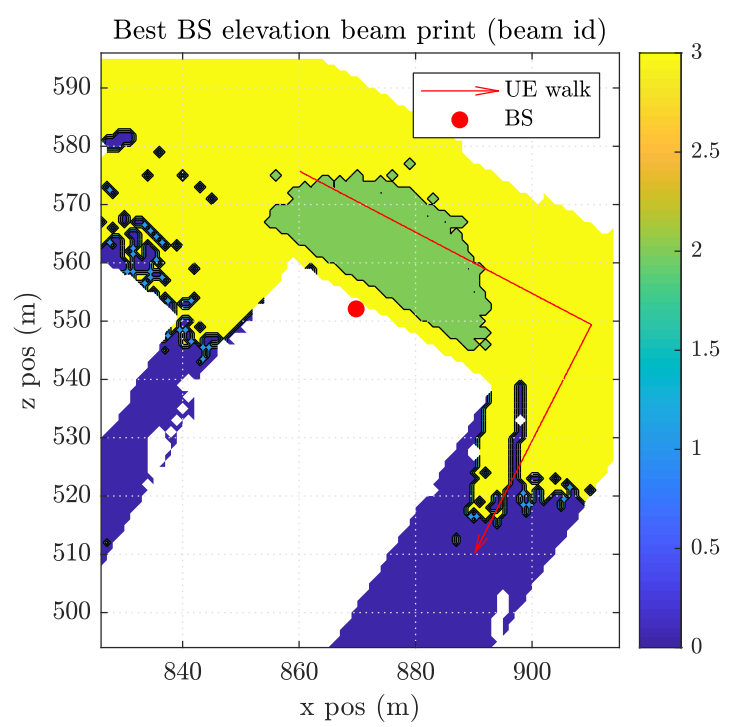

(b) Vertical beams

Figure 5.5: Beam prints of the gNB or BS at UE height in the evaluation region for UMa. The red arrow indicates the path and walk direction. 


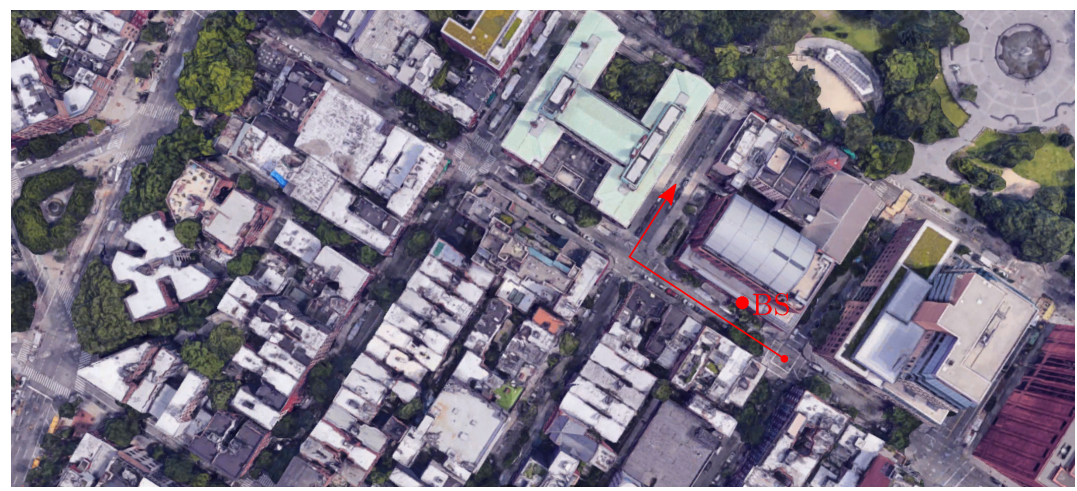

Figure 5.6: Aerial image from Google Maps of the zone considered in UMi. The location of the BS and the user walk trajectory are visually represented by a red dot and a red arrow, respectively.

\subsubsection{Tracking performance indicators}

For the sake of evaluating the performance of the beam tracking strategies, the following performance indicators are defined:

- The average throughput experienced by the UE during the simulation time, $t_{\text {simulation }}$.

- The beam alignment ratio, $\mathrm{R}_{\text {alignment }}$, can be defined as the amount of time the beam tracking strategy is suggesting using the optimal pair of beams, $t_{\text {alignment }}$, divided by the simulation time.

$$
\mathrm{R}_{\text {alignment }}=\frac{t_{\text {alignment }}}{t_{\text {simulation }}}
$$

Three beam alignment ratio are considered: the beam alignment ratio to the optimal pair of beams, the beam alignment ratio to the optimal gNB beam, and the beam alignment ratio to the optimal UE beam. It is worth noting that the beam alignment ratio is calculated from the beam pair evolution along the UE walk obtained with simulations in ns-3 with the Fifth Generation (5G) network performing a beam tracking strategy. That beam pair evolution obtained from simulations is compared to the optimal pair of beams analytically obtained from the channel equations and ray-tracing information. 


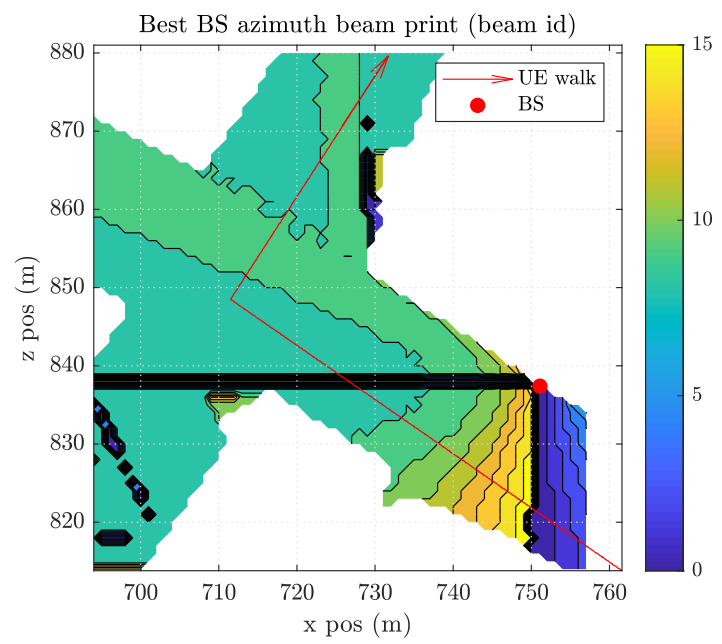

(a) Horizontal beams

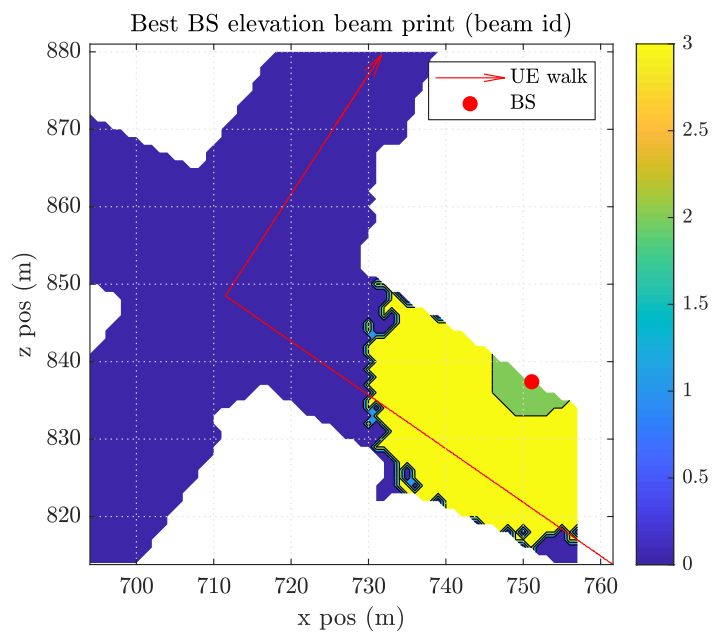

(b) Vertical beams

Figure 5.7: Beam prints of the gNB or BS at UE height in the evaluation region for UMi. The red arrow indicates the path and walk direction. 
- The Beam Gain Loss (BGL) is the average difference between the beam gain experienced in the UE walk at instant $t, G_{\text {current }}(t)$, and the beam gain obtained in case of using the optimal pair of beams, $G_{\text {optimal }}(t)$ :

$$
B G L=\overline{G_{\text {optimal }}(t)-G_{\text {current }}(t)}
$$

In case the currently-used beam pair is the optimal one, the difference is $0 \mathrm{~dB}$.

- The beam deviation percentage, which is the average probability of being different to the reference optimal beam pair. This beam deviation is expressed in azimuth and elevation domains.

- The beam distance is the difference in terms of beam ids between a reference beam $\mathrm{b}_{\mathrm{ref}}$ and a given beam $\mathrm{b}_{i}$ :

$$
\mathrm{d}=\left|\mathrm{b}_{\mathrm{ref}}-\mathrm{b}_{i}\right|
$$

Beam distances are calculated for each position in the area of interest's grid of points, and may refer to gNB or UE beams.

Moreover, beam distances may be expressed in azimuth and elevation beam distances. In this case, the absolute value operation in Equation 5.3 must be rewritten to support the modulus operation:

$$
\left(\mathrm{b}_{\text {ref }}-\mathrm{b}_{i}\right) \bmod N=\left(\mathrm{b}_{\text {ref }} \bmod N-\mathrm{b}_{i} \bmod N\right) \bmod N
$$

where $N$ is the number of beams of the considered domain. On the gNB side, $N=16$ in azimuth and $N=4$ in the elevation domain. Similarly, on the UE side, $N=8$ in azimuth and $N=2$ in the elevation domain.

\subsubsection{Baseline case definition}

When evaluating the system performance, it is a good practice to define a baseline case to serve as a reference. The baseline case can help gain understanding of the potential performance levels.

For beam tracking case, the adopted baseline case is set to perform beam tracking assuming full channel information is available at the network at each scheduling period, this is, every $1 \mathrm{~ms}$. Full channel knowledge implies knowing all the possible beam combinations between the gNB and the UE. In addition, no signalling overhead is assumed. This baseline case sets the maximum performance that can be achieved with beam tracking. 


\subsection{Tracking with SSB}

This first beam tracking strategy deals with achieving beam tracking with SSB. This is an immediate beam tracking approach since SSB signals are always transmitted with a given periodicity, for instance, a default periodicity value of $20 \mathrm{~ms}$ is assumed for initial access by UEs in NR Standalone (SA) deployments.

The advantage of a beam tracking strategy based on beam sweeping SSBs is that no extra signalling overhead for beam tracking is needed. One of the objectives of this section is to determine the effect of the SSB periodicity on the beam tracking performance. The objective is to find an optimum trade-off between the high availability of resources to transmit data with long periods, and the accurate beam tracking with short periods.

Section 5.2.1 presents some general considerations about tracking based on SSB, Section 5.2.2 addresses the study of the UMa scenario, while Section 5.2.3 is focused on the UMi scenario.

\subsubsection{General considerations}

This section clarifies which is the maximum expected delay from the moment in which a change of the optimal beam pair between a BS and a UE occurs to the time when the beam tracking based on SSB captures that change.

Recall that the SS burst periodicities may be $5 \mathrm{~ms}, 10 \mathrm{~ms}, 20 \mathrm{~ms}, 40 \mathrm{~ms}$, $80 \mathrm{~ms}$ or $160 \mathrm{~ms}$. In any case, the SSB signals are sent during the first $5 \mathrm{~ms}$ of that period. During those first $5 \mathrm{~ms}$ the BS completes its beam sweeping cycle, i.e., during that $5 \mathrm{~ms}$ period all the BS beams are used to beamform different SSBs in different spatial directions. To complete the beam sweeping by the UE, as many SS bursts as UE beams will be necessary.

Based on those characteristics, the worst case in terms of the amount of time needed to detect a change of beam pair involves two conditions: (i) the change implies that the new best pair is the first beam in an SS burst, and (ii) that beam has just beam measured. In that case, the time to detect the change is the time to perform a complete beam sweeping cycle of the UE plus the duration of an SS burst.

Table 5.2 lists the maximum beam update delays for the different values of SS burst periods assuming the UE uses 16 beams. The maximum delays have also been translated to distances covered in case of a pedestrian speed of $1 \mathrm{~m} / \mathrm{s}$ and a vehicular speed of $15 \mathrm{~m} / \mathrm{s}$. 
Table 5.2: Maximum time delay to update the pair of beams with SSB beam sweeping. It is assumed that the UE has 16 beams and the gNB transmits in all its beams during one SS burst.

\begin{tabular}{|r|r|r|r|}
\hline \multirow{2}{*}{$\begin{array}{c}\text { SS burst } \\
\text { period (ms) }\end{array}$} & \multirow{2}{*}{$\begin{array}{c}\text { Maximum } \\
\text { delay (s) }\end{array}$} & \multicolumn{2}{|c|}{ Distance for max. delay (m) } \\
\cline { 3 - 4 } & Pedestrian & \multicolumn{1}{c|}{ Vehicle } \\
\hline \hline 5 & 0.085 & 0.085 & 1.275 \\
\hline 10 & 0.165 & 0.165 & 2.475 \\
\hline 20 & 0.325 & 0.325 & 4.875 \\
\hline 40 & 0.645 & 0.645 & 9.675 \\
\hline 80 & 1.285 & 1.285 & 19.275 \\
\hline 160 & 2.565 & 2.565 & 38.475 \\
\hline
\end{tabular}

\subsubsection{Urban macro scenario}

As the UE moves on its defined path, the optimal pair of gNB and UE beams changes. Figures 5.8 and Figure 5.9 aim at illustrating the temporal evolution of the gNB and UE beam ids used during the whole walk at pedestrian and vehicular speeds respectively. The solid black line curve represents the beam pair evolution obtained with the baseline case. The rest of curves represent the evolution of the beam ids obtained with SSB beam tracking and different SS burst periods ranging from $5 \mathrm{~ms}$ to $160 \mathrm{~ms}$. The greyed areas starting at $t=68 \mathrm{~s}$ in Figure 5.8 and $t=4.4 \mathrm{~s}$ in Figure 5.9 correspond to the NLoS areas, where the LoS ray is obstructed. Note that in these two figures, and in the remaining figures in this Thesis illustrating the temporal beam id evolution, the stationary regime transition time of 3 seconds is excluded.

Starting with the mobility case at pedestrian speed illustrated in Figure 5.8, performing beam sweeping with the most frequent SS burst configurations (up to $40 \mathrm{~ms}$ periodicity) provides good beam tracking performance, practically matching the beam pair evolution of the baseline case. However, the longer SS burst configuration may introduce significant update delays with respect to the baseline case.

It is true that beam tracking by performing beam sweeping of SSB may fail in capturing very quick beam pair fluctuations. This is the case of the pedestrian mobility case at walk point 48 metres, where the scenario significantly distorts the beam prints and a different optimal beam pair appeared, in case of using periodicities of $80 \mathrm{~ms}$ or $160 \mathrm{~ms}$. The impossibility of capturing very fast fluctuations with SSB-only beam tracking is clear in Figure 5.8(d), in which the UE elevation beam fluctuates very fast. This is motivated by the fact that, 

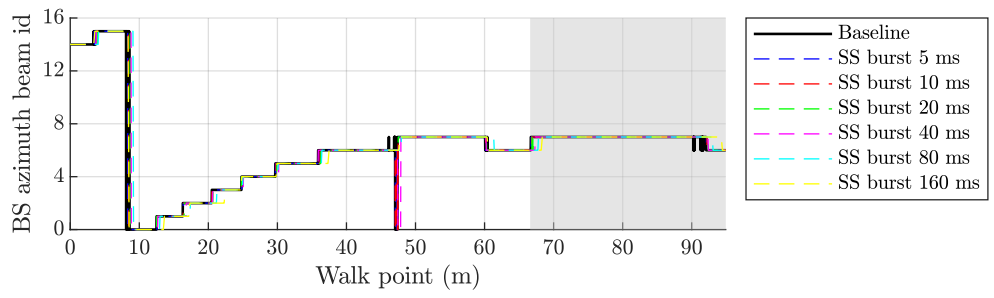

(a) gNB azimuth beam id.
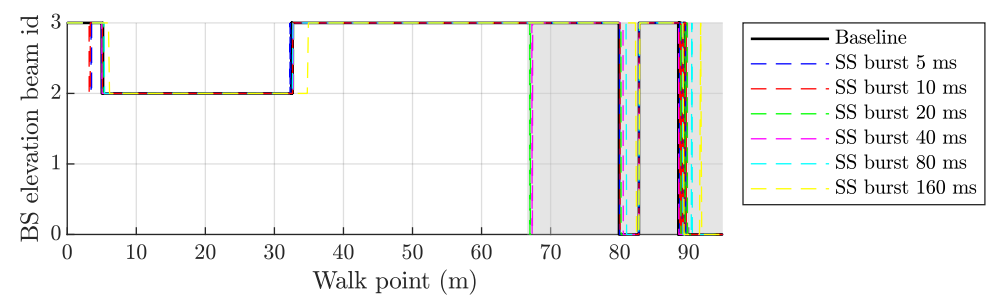

(b) gNB elevation beam id.
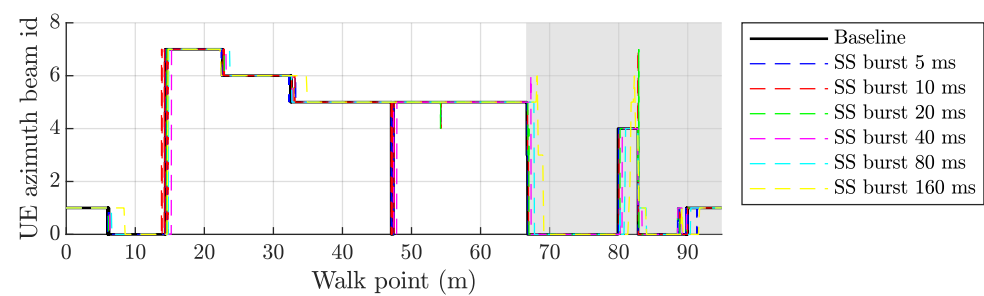

(c) UE azimuth beam id.
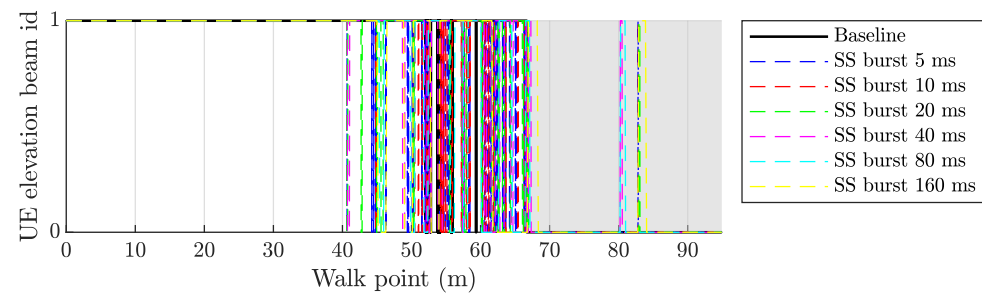

(d) UE elevation beam id.

Figure 5.8: Beam ids changes in UMa walk at pedestrian speed. The greyed area corresponds to NLoS region. 

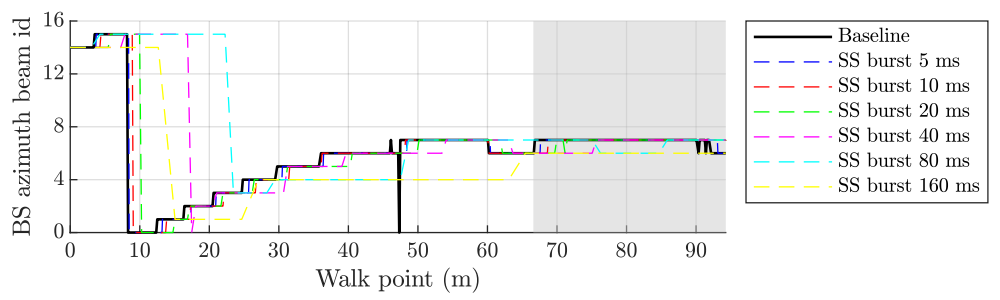

(a) gNB azimuth beam id.

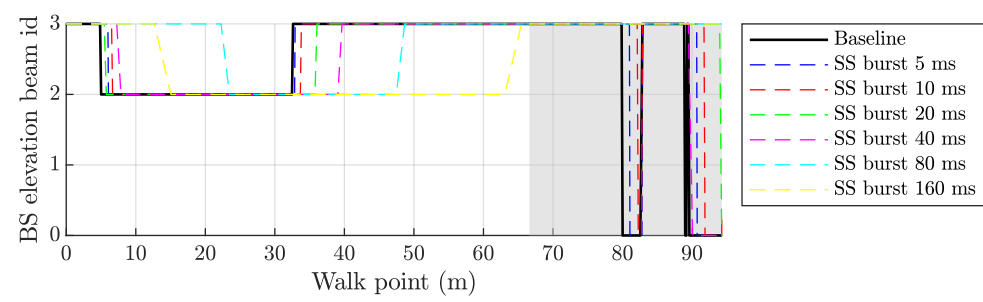

(b) gNB elevation beam id.
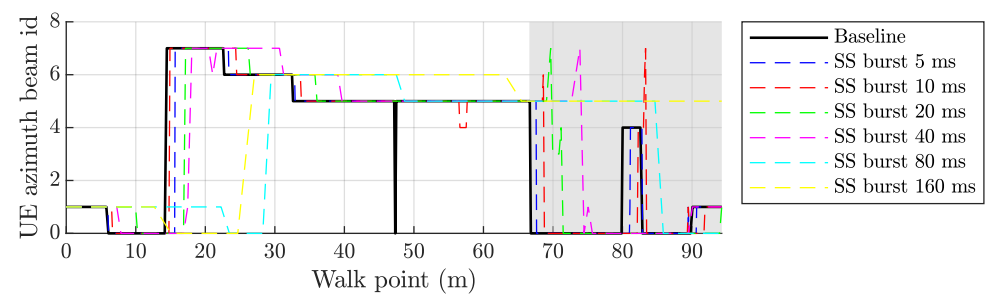

(c) UE azimuth beam id.
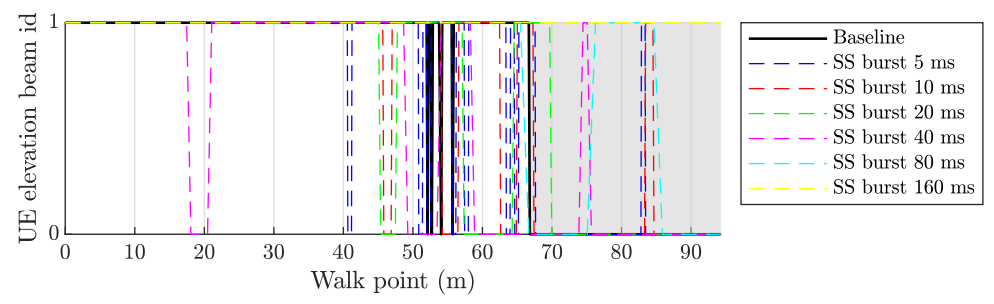

(d) UE elevation beam id.

Figure 5.9: Beam id changes in UMa walk at vehicular speed. The greyed area corresponds to NLoS region. 


\section{CHAPTER 5. BEAM TRACKING TECHNIQUES}

although having only 2 beams in elevation, the beam gain obtained with both elevation beams is very similar in a significant part of the route.

Concerning the vehicular case, Figure 5.9 shows the beam id changes as a function of the distance covered. The delays in Figure 5.9 are appreciated easier than in the pedestrian speed case. The reason is that, although the maximum adaptation delays are the same, the distance covered by a vehicle during the adaptation period is longer. As a result, the beam misalignments can be observed already for a period of $5 \mathrm{~ms}$. In any case, the duration of beam misalignments is always within the limits shown in Table 5.2. Note, also, that for periods of $5 \mathrm{~ms}$ and $10 \mathrm{~ms}$ the misalignment is very small except in the case of UE elevation where as in the pedestrian case fast artificial variations are produced.

The beam tracking ability determines the experienced Signal to Interference plus Noise Ratio (SINR) as illustrated in Figure 5.10(a) and Figure 5.10(b) for the pedestrian and vehicular mobility cases.

In Figure 5.10(a), for the pedestrian case, the experienced SINR is high in LoS due to the UE proximity to the gNB antenna panel. In this region, the SSB-based beam tracking with longest SS burst period presents ripple due to the late beam update to the optimal value, but still the SINR remains high. In this particular case, the beam misalignments are not severe, given the high SINR. This fact indicates that the beams used before the beam update still provide good beamforming gains. Due to blockage, the NLoS regions present a reduced SINR, with a drop of about $35 \mathrm{~dB}$ in the transition from LoS to NLoS.

In the vehicular mobility case in Figure 5.10(b), the beam adaptation delay affects the SINR more critically than in the pedestrian speed case, specially with the longest SS burst period. For a given adaptation delay, the beam misalignment may be higher. Therefore, the beam gain can be much lower compared to the optimal case and, therefore, the SINR may also be significantly lower.

As the SINR is affected by the beam tracking ability, so is the experienced throughput. Figure 5.11(a) illustrates the temporal evolution of the throughput experienced by a user during its pedestrian walk with the network performing beam tracking based on SSB beam sweeping only. As it can be seen, the selection of the SS burst period determines the maximum achievable throughput. As discussed in Section 2.5, the selection of the SS burst period influences on the signalling overhead, and the longest period of $160 \mathrm{~ms}$ provides the smallest contribution to overhead. In an opposite situation, the shortest period of $5 \mathrm{~ms}$ provides the largest overhead. In the throughput stationary area in LoS, the difference between $5 \mathrm{~ms}$ and $160 \mathrm{~ms}$ is $50 \mathrm{Mbps}$. More importantly, enlarging the SS burst period allows reaching the throughput in the baseline case, 


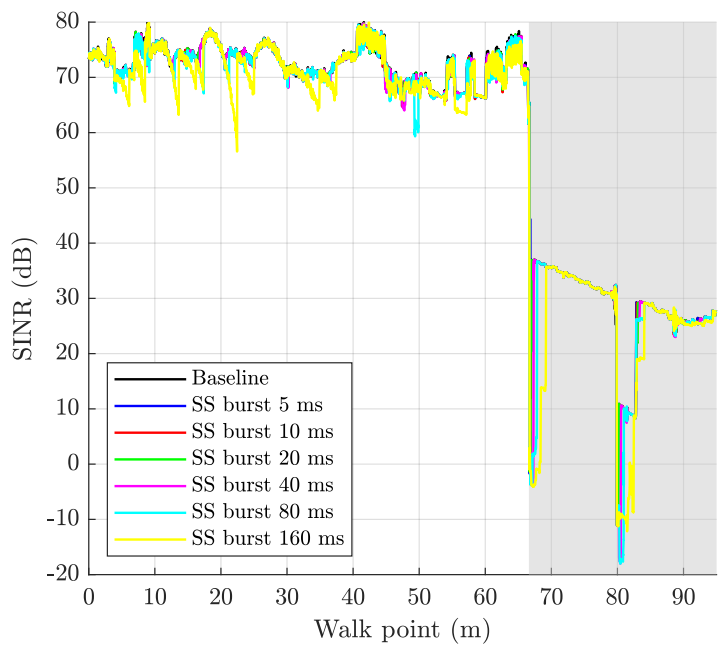

(a) Pedestrian speed.

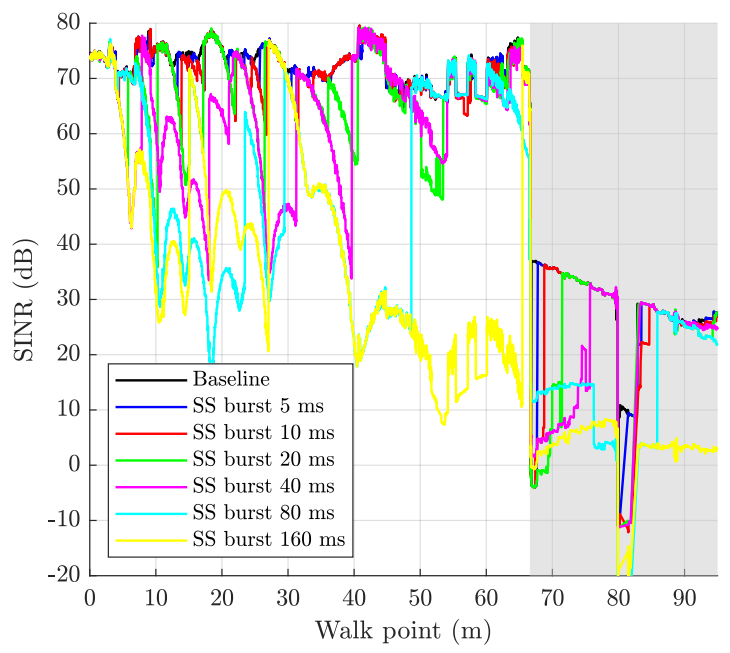

(b) Vehicular speed.

Figure 5.10: Temporal evolution of the SINR in the UMa walk for different speeds. Greyed areas correspond to a NLoS situation. 


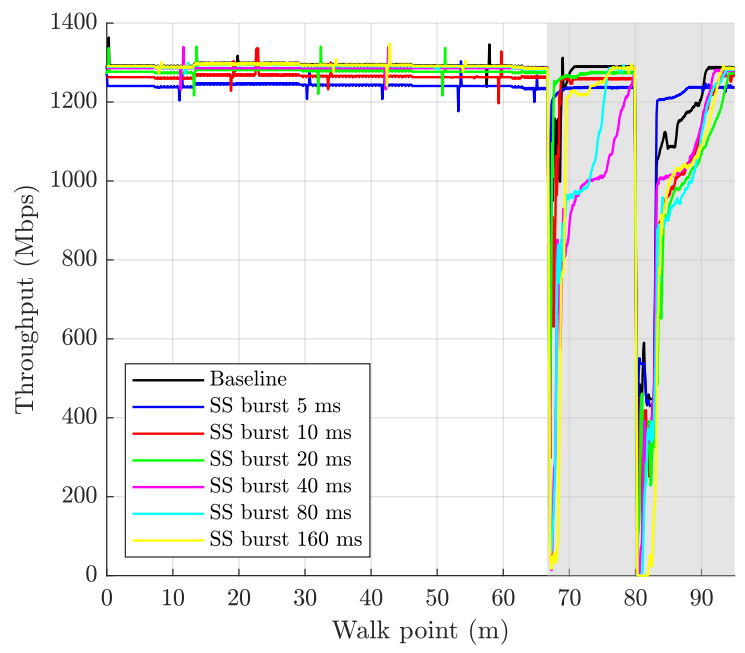

(a) Pedestrian speed

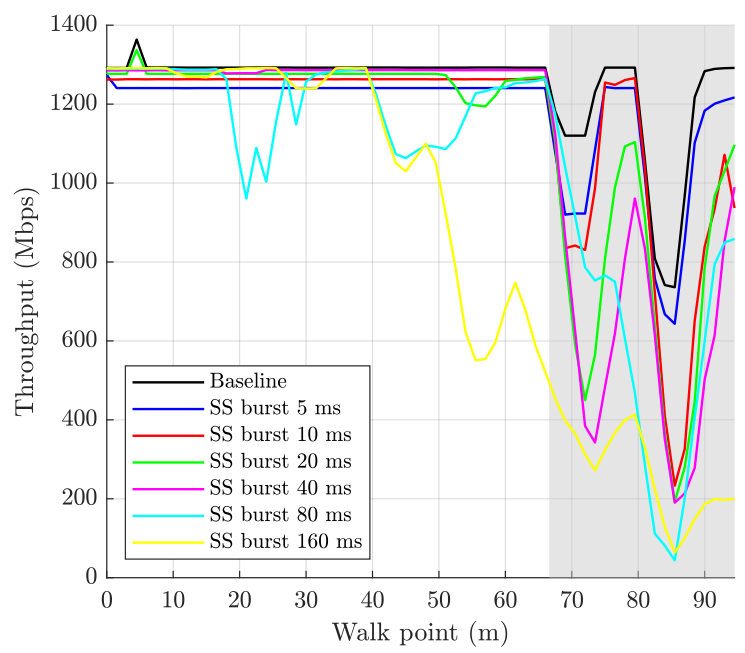

(b) Vehicular speed.

Figure 5.11: Temporal evolution of the user throughput experienced in the UMa walk for different speeds. Greyed areas correspond to a NLoS situation. 
which assumes complete beam pair information every $1 \mathrm{~ms}$ with no signalling overhead.

In the NLoS area, however, the throughput experienced with the longer SS burst periods can be worse than with the shorter periods due to, again, the delayed beam updates to the optimal beams. Once the optimal beam pair has been found, the data rate is recovered and practically reaches the baseline case.

The vehicular mobility case provides a different experience. As it can be seen in Figure 5.11(b), the instant throughput experienced with delayed beam updates is negatively impacted by beam misalignmets. As expected, the largest SS burst configuration is critically affected, even in LoS, due to strong SINR drops.

The obtained average throughput values are summarised in Table 5.3. In NLoS regions, the lowest SS burst period of $5 \mathrm{~ms}$ is the one providing optimum results, both at pedestrian and vehicular speeds. We observe, as expected from the SINR and throughput evolution graphics, that the throughput decreases rapidly as the SS burst period increases. In LoS the optimum value depends on the speed. For pedestrian speed, it is better to consider the longer period which minimises the amount of resources devoted to reference signals because all the periods are providing a similar tracking of the channel. For vehicular speed, a period of $40 \mathrm{~ms}$ is found to be the best trade-off between minimising the reference signals overhead and having and tracking the best beam pair accurately. Globally considering the whole path, see "All" column, the conclusion for NLoS holds, i.e. a period of $5 \mathrm{~ms}$ is the one providing optimum global results.

The beam alignment ratio achieved in the UMa scenario is summarised in Table 5.4. In general terms, the best beam tracking ratio is obtained with the shortest SS burst periods of $5 \mathrm{~ms}$. Enlarging the periodicity reduces the beam tracking ability since beam updates are obtained delayed in time. As a consequence, the beam alignment ratio reduces as the SS burst period is increased. The difference between SS burst periods is more accentuated at vehicular speeds, due to the longer distance covered in a beam misalignment situation. The best beam tracking ability is obtained with an SS burst period $5 \mathrm{~ms}$, which obtained a beam alignment probability of $86 \%$. On the other hand, the longest SS burst period value of $160 \mathrm{~ms}$ provides the worst beam tracking performance: a $22 \%$.

Finally, the average BGL values experienced in dB for both mobility speeds and the different SS burst periods in consideration are also included in Table 5.4. In general, the longest the beam misalignment, the largest the BGL. This fact can be appreciated when comparing the values obtained in the pedestrian mobility case with the short and long SS burst periods, or when comparing the values obtained for the two mobility speeds for a fixed SS burst period. 
Table 5.3: Average throughput achieved with SSB beam tracking in UMa.

\begin{tabular}{|c|c|c|c|c|}
\hline \multirow{2}{*}{ Speed } & \multirow{2}{*}{$\begin{array}{c}\text { SS burst } \\
\text { period }(\mathrm{m} / \mathrm{s})\end{array}$} & \multicolumn{3}{|c|}{ Average Throughput (Mbps) } \\
\hline & & All & LoS & NLoS \\
\hline \multirow{7}{*}{ Pedestrian } & Baseline & 1249.40 & 1294.09 & 1145.02 \\
\hline & 5 & 1213.17 & 1242.02 & 1145.79 \\
\hline & 10 & 1206.15 & 1265.15 & 1068.29 \\
\hline & 20 & 1211.43 & 1278.58 & 1054.51 \\
\hline & 40 & 1186.98 & 1285.72 & 956.22 \\
\hline & 80 & 1191.77 & 1290.99 & 959.95 \\
\hline & 160 & 1195.75 & 1292.53 & 969.60 \\
\hline \multirow{7}{*}{ Vehicular } & Baseline & 1242.54 & 1294.10 & 1120.41 \\
\hline & 5 & 1181.40 & 1241.40 & 1039.29 \\
\hline & 10 & 1150.37 & 1262.97 & 883.68 \\
\hline & 20 & 1115.02 & 1269.45 & 749.26 \\
\hline & 40 & 1085.75 & 1285.22 & 613.31 \\
\hline & 80 & 1031.52 & 1211.73 & 604.69 \\
\hline & 160 & 852.05 & 1098.85 & 267.52 \\
\hline
\end{tabular}

Table 5.4: Beam alignment ratio and BGL in UMa walk for different SS burst periods and UE speeds

\begin{tabular}{|c|r|r|r|r|r|}
\hline \multirow{3}{*}{ Speed } & \multirow{2}{*}{$\begin{array}{c}\text { SS burst } \\
\text { period (ms) }\end{array}$} & \multicolumn{3}{|c|}{$\mathbf{R}_{\text {alignment }}$} & BGL \\
\cline { 3 - 6 } & & Pair & gNB & UE & (dB) \\
\hline \hline \multirow{4}{*}{ Pedestrian } & 5 & 0.94 & 0.98 & 0.95 & 1.79 \\
\cline { 2 - 6 } & 10 & 0.92 & 0.97 & 0.94 & 2.24 \\
\cline { 2 - 6 } & 20 & 0.90 & 0.96 & 0.93 & 2.58 \\
\cline { 2 - 6 } & 40 & 0.86 & 0.93 & 0.91 & 3.43 \\
\cline { 2 - 6 } & 80 & 0.79 & 0.89 & 0.86 & 4.00 \\
\hline \hline \multirow{4}{*}{ Vehicular } & 160 & 0.62 & 0.81 & 0.74 & 4.78 \\
\cline { 2 - 6 } & 5 & 0.86 & 0.92 & 0.92 & 4.12 \\
\cline { 2 - 6 } & 10 & 0.73 & 0.83 & 0.84 & 4.85 \\
\cline { 2 - 6 } & 20 & 0.61 & 0.74 & 0.72 & 5.53 \\
\cline { 2 - 6 } & 40 & 0.51 & 0.62 & 0.63 & 7.81 \\
\cline { 2 - 6 } & 80 & 0.38 & 0.54 & 0.46 & 11.43 \\
\cline { 2 - 6 } & 160 & 0.22 & 0.38 & 0.26 & 14.73 \\
\hline
\end{tabular}


These two parameters seem to be correlated and, as one value increases, the other decreases.

\subsubsection{Urban micro scenario}

The main difference between UMi scenario and the previously analysed UMa scenario is the antenna height and the area of interest where the performance of the beam tracking strategies are to be evaluated. In the first place, the evolution of the beam ids throughout the UE walk in the UMi scenario is to be studied.

For that aim, and with the intention of serving as a visual comparison, Figure 5.12 and Figure 5.13 shows the temporal evolution of the serving gNB and UE beam ids as the UE moves along the predefined walk at pedestrian and vehicular speeds respectively. The solid black line represents the beam pair evolution in the baseline case, which serves as visual reference. The coloured dashed curves correspond to the evolution of the beam ids obtained with SSB beam tracking and different SS burst periods. The NLoS region starts 67 metres away from the starting point. This region is shown in both figures with a greyed colour in background.

Starting with the mobility case at pedestrian speed illustrated in Figure 5.12, performing beam sweeping with an SS burst period of $40 \mathrm{~ms}$ or less provides an almost perfect tracking of the BS azimuth beam id and very short misalignments for the BS elevation beam id and for the UE beam ids (both in azimuth and elevation).

Figure 5.13 illustrates the temporal evolution of the beam ids obtained from SSB-only beam tracking at vehicular speed. As occurred in the UMa scenario, the beam tracking delays are more noticeable for the vehicular speed. We can see that the maximum delays reported in Section 5.2.1 are fulfilled with some deviations around the walk point at $40 \mathrm{~m}$ (see the variation of the BS elevation beam id).

The beam misalignments determine the experienced SINR as illustrated in Figure 5.14(a) for the pedestrian mobility case and in Figure 5.14(b) for the vehicular mobility case. At the beginning of the walk, the UE is situated very close to the gNB antenna panel. This explains the large SINR values in the LoS region when beam pairs are in alignment. As the SS burst period increases, beam misalignment situation are larger in space and, as a consequence, the user experiences more areas with reduced SINR values. Note that the SSBbased beam tracking with longest SS burst period presents the deepest signal fluctuations due to the late beam update to the optimal value in pedestrian and, specially, in vehicular mobility. Due to different blockage situations in the NLoS region, the SINR level progressively degrades within two steps. A 

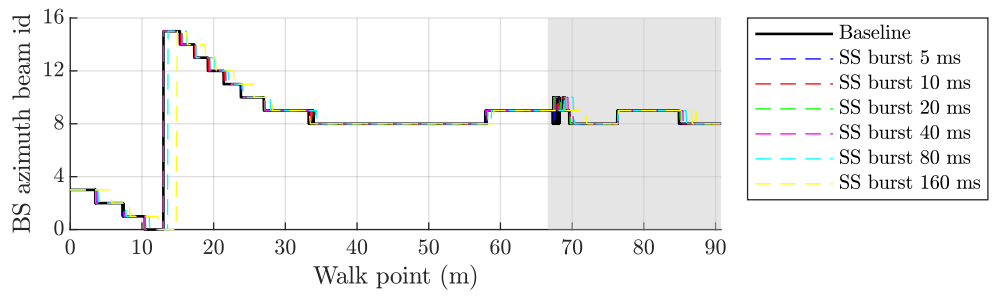

(a) gNB azimuth beam id.
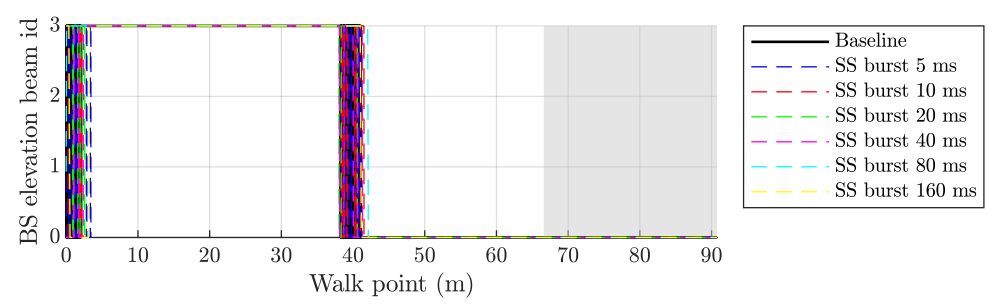

(b) gNB elevation beam id.
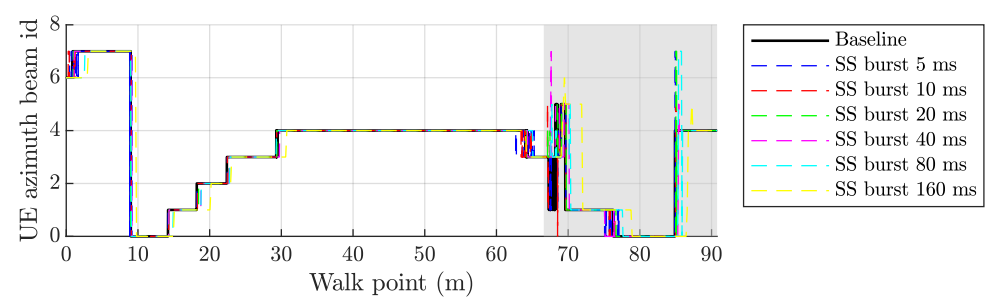

(c) UE azimuth beam id.
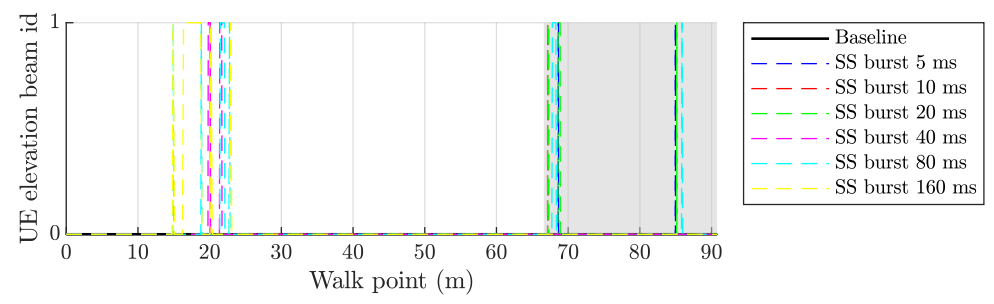

(d) UE elevation beam id.

Figure 5.12: Beam id changes in UMi walk at pedestrian speed. Greyed background areas indicates NLoS situation. 

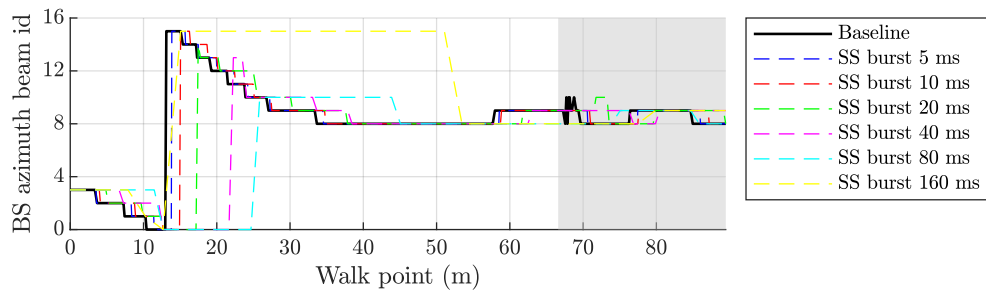

(a) gNB azimuth beam id.
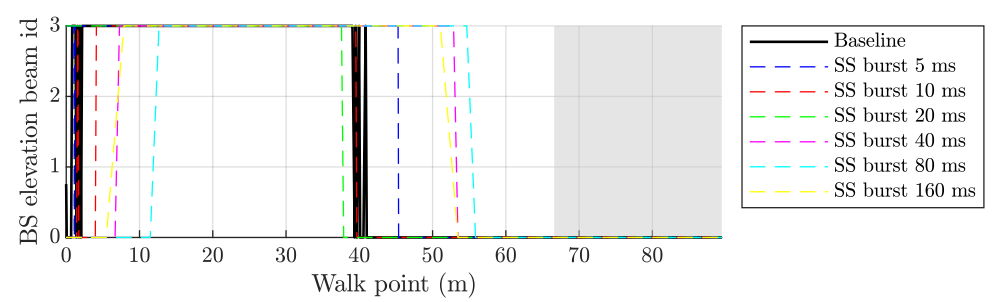

(b) gNB elevation beam id.
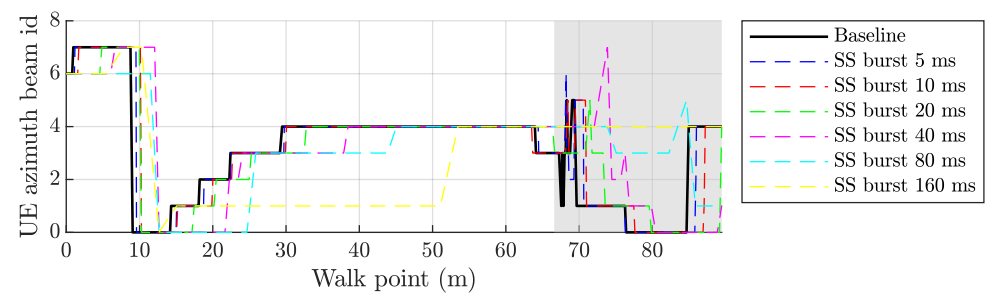

(c) UE azimuth beam id.
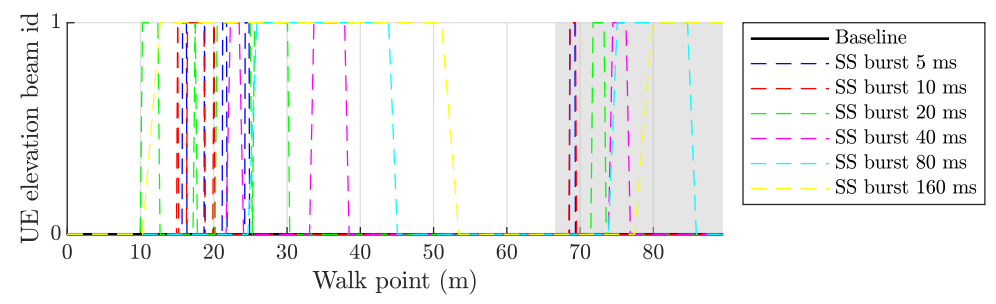

(d) UE elevation beam id.

Figure 5.13: Beam id changes in UMi walk at vehicular speed. Greyed background areas indicates NLoS situation. 


\section{CHAPTER 5. BEAM TRACKING TECHNIQUES}

first drop of about $30 \mathrm{~dB}$ occurs in the transition from LoS to NLoS when the user has walked 67 metres. This drop is motivated by the fact of rays arriving with one reflection as minimum. There is a second deep SINR drop 85 metres away from the starting point. In this second step, the minimum number of reflections of rays arriving at the UE position is 2, which explains the SINR level reduction, which barely provides $10 \mathrm{~dB}$. Such SINR level is insufficient for maintaining high data speed connections, and a handover to a different BS should be encouraged to boost the throughput. Nevertheless, the consideration of multiple BSs is out of the scope of this Thesis.

In the vehicular mobility case, beam misalignments are more significant due to the further distance travelled in a beam misalignment situation. Naturally, this fact has a strong influence on the experienced SINR as illustrated in Figure 5.14(b), specially with the longest SS burst period, which experiences a SINR level below $0 \mathrm{~dB}$ at the end of the simulation.

The throughput experienced by the UE is largely determined by the experienced SINR. Figure 5.15(a) illustrates the temporal evolution of the throughput experienced by the user during its walk with the network performing beam tracking based on SSB beam sweeping only. The selection of the SS burst period determines the signalling overhead and determines the peak data rate. In general, in LoS, the throughput is higher with the largest SS burst period than with the shortest SS burst period. More importantly, enlarging the SS burst period allows reaching the baseline case in LoS.

After a recovering from a significant drop of the experienced SINR value, the throughput rate is recovered. But the recovery speed may vary and does not exclusively depend of the SINR value or the amount of time/frequency resources available. The TCP layer has a lot to say in this recovery and, more precisely, the Congestion Window $(\mathrm{CW})$. Figure 5.16 depicts the CW status 20 metres after and before the LoS to NLoS transition. It can be seen that after the walk point 70 metres, the $\mathrm{CW}$ shuts and its size is reduced, and so is the number of packets transmitted. The CW associated to each SS burst period curve opens differently: in this example, with periods of $20 \mathrm{~ms}, 80 \mathrm{~ms}$ and $160 \mathrm{~ms}$ the $\mathrm{CW}$ is practically completely closed, and 5 metres later starts opening up slowly.

Figure 5.17 depicts the Round Trip Time (RTT). It can be seen that after the walk point 70 metres, the SS burst period curves remain flat for some space and at different RTT values. But this is related to the CW and the throughput, which is larger in the baseline. Note that during the time the $\mathrm{CW}$ is closed, the RTT values are fixed, meaning that the number of packets in the transmission buffer size is maintained.

In the vehicular mobility case illustrated in Figure 5.15(b), it can be seen that the instant throughput experienced with delayed beam updates is neg- 


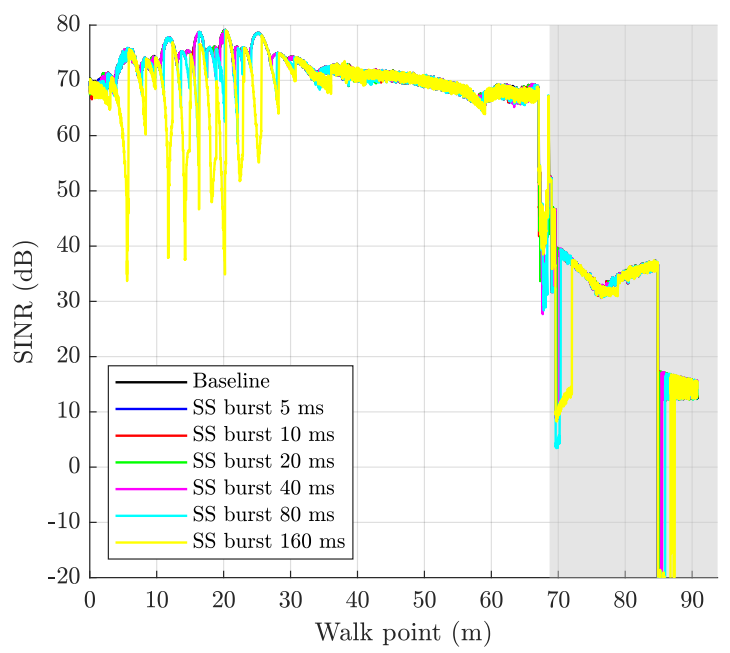

(a) Pedestrian speed.

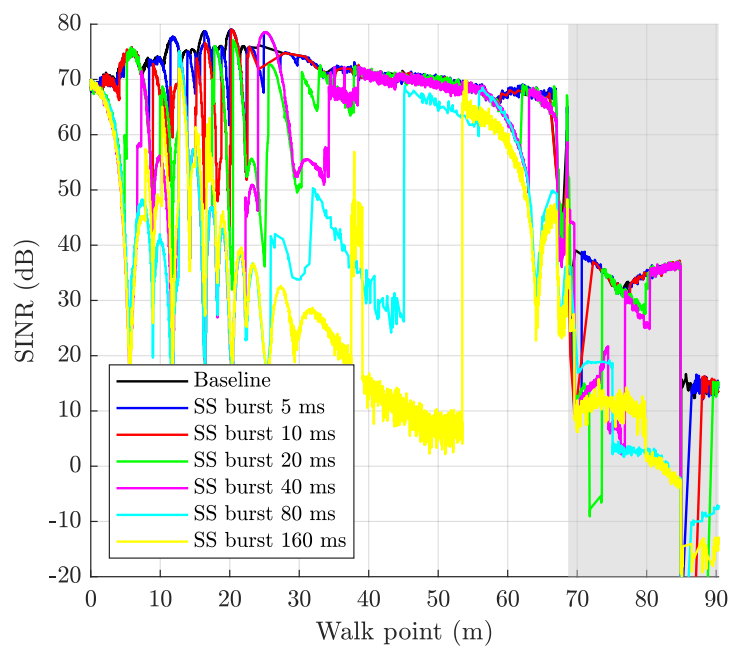

(b) Vehicular speed.

Figure 5.14: Temporal evolution of the SINR in the UMi walk for different speeds. Greyed areas correspond to a NLoS situation. 


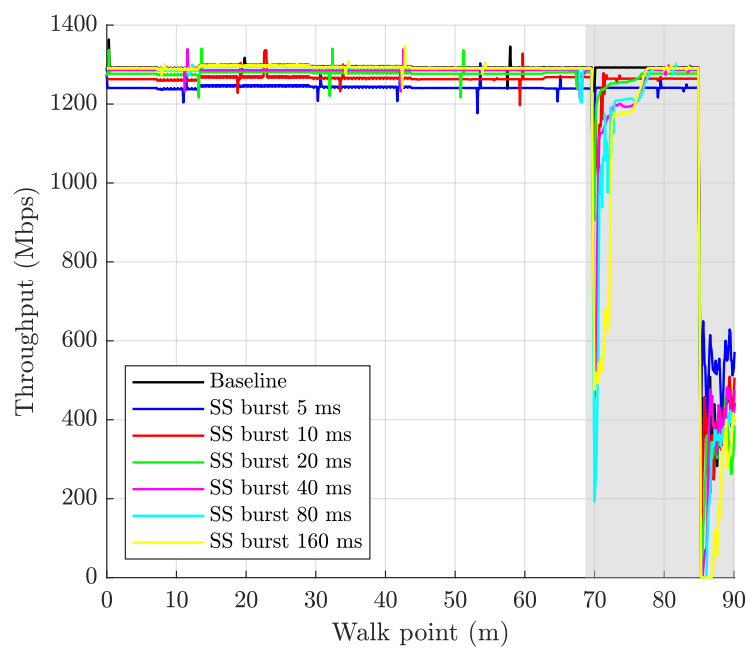

(a) Pedestrian speed

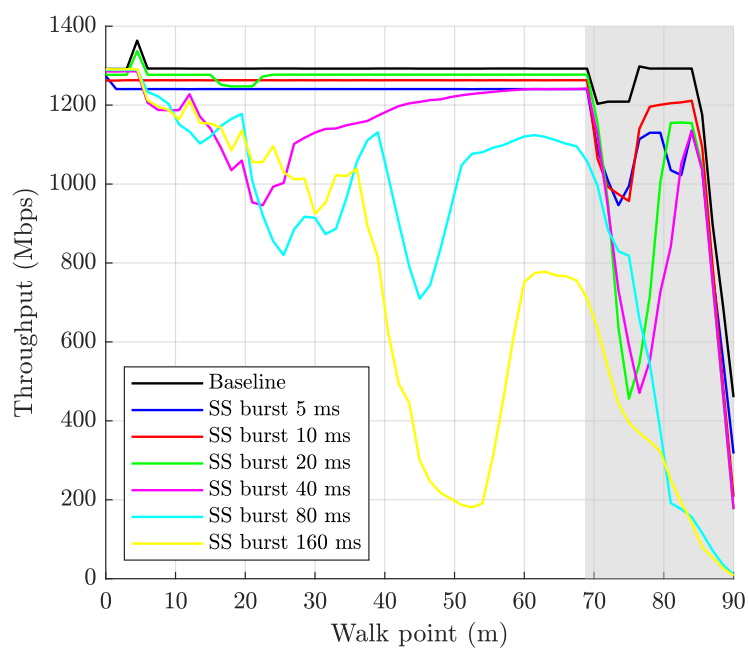

(b) Vehicular speed.

Figure 5.15: Temporal evolution of the user throughput experienced in the UMi walk for different speeds. Greyed areas correspond to a NLoS situation. 


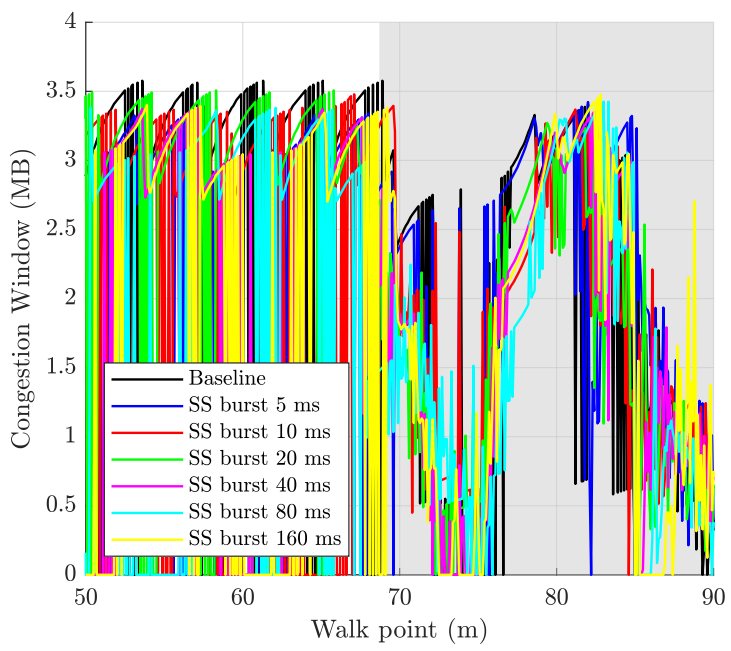

Figure 5.16: Congestion window status in the UMi walk at pedestrian speed for the SSB beam sweeping with the two SS burst periods.

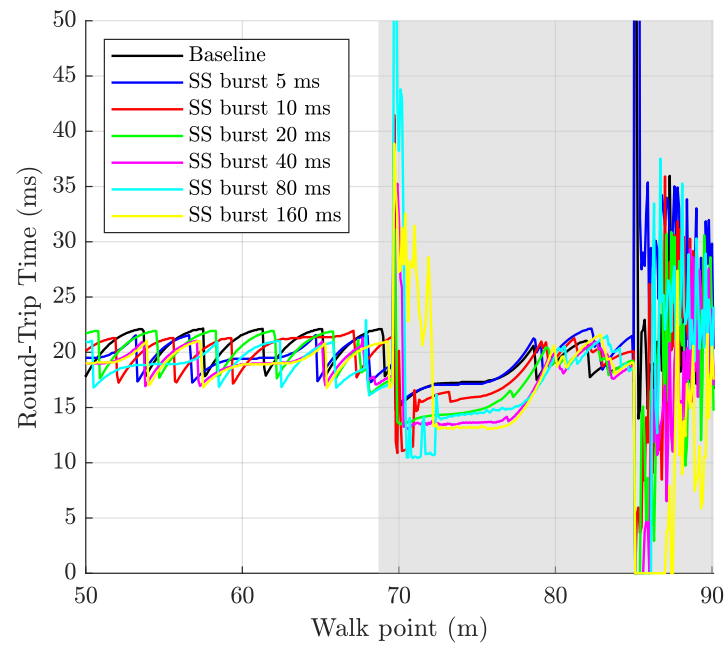

Figure 5.17: Round-Trip Time in ms in the UMi walk at pedestrian speed for the SSB beam sweeping with the two SS burst periods. 


\section{CHAPTER 5. BEAM TRACKING TECHNIQUES}

atively impacted by beam misalignmets. SS burst configurations larger than $40 \mathrm{~ms}$ are critically affected, even in LoS, due to strong SINR drops. And the throughput degradation is stronger as the period increases. Focusing on NLoS, the degradation compared to the baseline is noticeable even for the lower periods (around $10 \%$ for periods of $5 \mathrm{~ms}$ and $10 \mathrm{~ms}$ ). As a result, the performance of the beam tracking strategy based on SSB beam sweeping seems to be improvable, and alternative beam tracking strategies or mechanisms should be considered.

The obtained average throughput values are summarised in Table 5.5. As expected from the visual inspection of the throughput figures, the largest data rate values are obtained at pedestrian speed. Enlarging the SS burst period increases the average throughput experienced during the pedestrian walk in LoS conditions given that in this case the beam tracking is very good for all periods and it is more important to reduce the signalling overhead. However, the throughput decreases in NLoS as the SS burst period increases since in that case the beam misalignments due to higher periods have a very detrimental effect.

These trends are not equal at vehicular speeds. In that case, the throughput increases if the period increases initially, up to a period of $20 \mathrm{~ms}$ in LoS and up to $10 \mathrm{~ms}$ in NLoS, but decreases for larger periods. In LoS, the reason is that for vehicular speeds the misalignments are significant and hence, there is a trade-off between minimising the signalling overhead and reducing the beam misalignments. In NLoS, the trend deviation is very small and can be explained by some random effects derived from the TCP protocol due to large signal fading.

As in the UMa case, a good general selection to obtain good results, close to optimum both in LoS and NLoS, is the periodicity of $5 \mathrm{~ms}$. In this case, also a value of $10 \mathrm{~ms}$ would be a good selection for the two speeds considered, while $20 \mathrm{~ms}$ would be acceptable only for pedestrian speed.

The beam alignment ratio achieved in the UMi scenario is summarised in Table 5.6. In general, the beam tracking is best with the shortest SS burst period of $5 \mathrm{~ms}$, and enlarging the periodicity reduces the tracking performance since beam updates are obtained later. The beam alignment ration difference between pedestrian and vehicular speeds ranges between $16 \%$ with the shortest SS burst period and $60 \%$ for the longest SS burst period.

Finally, Table 5.6 summarises the average BGL values for the two mobility speeds and the multiple SS burst periods under evaluation. In general, the longest the beam misalignment, the largest the beam gain loss. 
Table 5.5: Average throughput achieved with SSB beam tracking in UMi.

\begin{tabular}{|c|r|r|r|r|}
\hline \multirow{2}{*}{ Speed } & \multicolumn{1}{|c|}{$\begin{array}{c}\text { SS burst } \\
\text { period } \mathbf{( m / s )}\end{array}$} & \multicolumn{3}{|c|}{ Average Throughput (Mbps) } \\
\cline { 3 - 5 } & Baseline & 1243.38 & 1294.26 & 1080.69 \\
\hline \hline \multirow{5}{*}{ Pedestrian } & 5 & 1200.93 & 1242.18 & 1069.12 \\
\cline { 2 - 5 } & 10 & 1211.77 & 1265.48 & 1040.14 \\
\cline { 2 - 5 } & 20 & 1220.08 & 1278.61 & 1033.07 \\
\cline { 2 - 5 } & 40 & 1218.61 & 1285.24 & 1005.69 \\
\cline { 2 - 5 } & 80 & 1215.53 & 1290.72 & 975.29 \\
\cline { 2 - 5 } & 160 & 1203.72 & 1292.73 & 919.31 \\
\hline \hline \multirow{5}{*}{ Vehicular } & Baseline & 1256.28 & 1294.07 & 1132.04 \\
\cline { 2 - 5 } & 5 & 1174.31 & 1241.39 & 968.61 \\
\cline { 2 - 5 } & 10 & 1198.20 & 1262.98 & 999.57 \\
\cline { 2 - 5 } & 20 & 1169.38 & 1275.83 & 842.94 \\
\cline { 2 - 5 } & 40 & 1080.51 & 1175.63 & 788.80 \\
\cline { 2 - 5 } & 80 & 904.90 & 1049.57 & 461.24 \\
\cline { 2 - 5 } & 160 & 710.01 & 843.55 & 300.50 \\
\hline
\end{tabular}

Table 5.6: Beam alignment ratio and BGL in UMi walk for different SS burst periods and UE speeds

\begin{tabular}{|c|r|r|r|r|r|}
\hline \multirow{3}{*}{ Speed } & \multirow{2}{*}{$\begin{array}{c}\text { SS burst } \\
\text { period (ms) }\end{array}$} & \multicolumn{3}{|c|}{$\mathbf{R}_{\text {alignment }}$} & BGL \\
\cline { 3 - 6 } & Pair & gNB & UE & (dB) \\
\hline \hline \multirow{4}{*}{ Pedestrian } & 5 & 0.93 & 0.94 & 0.98 & 1.65 \\
\cline { 2 - 6 } & 10 & 0.91 & 0.93 & 0.97 & 1.77 \\
\cline { 2 - 6 } & 20 & 0.89 & 0.92 & 0.96 & 2.00 \\
\cline { 2 - 6 } & 40 & 0.86 & 0.91 & 0.93 & 2.86 \\
\cline { 2 - 6 } & 80 & 0.76 & 0.84 & 0.88 & 3.02 \\
\hline \multirow{4}{*}{ Vehicular } & 160 & 0.62 & 0.70 & 0.81 & 4.34 \\
\cline { 2 - 6 } & 5 & 0.68 & 0.72 & 0.93 & 2.47 \\
\cline { 2 - 6 } & 10 & 0.62 & 0.67 & 0.87 & 3.28 \\
\cline { 2 - 6 } & 20 & 0.41 & 0.50 & 0.70 & 4.03 \\
\cline { 2 - 6 } & 40 & 0.36 & 0.41 & 0.63 & 5.26 \\
\cline { 2 - 6 } & 80 & 0.16 & 0.34 & 0.45 & 6.92 \\
\cline { 2 - 6 } & 160 & 0.14 & 0.26 & 0.32 & 9.53 \\
\hline
\end{tabular}




\subsection{Tracking with SSB and CSI-RS}

Once the UE successfully completes the initial access procedure, the network may assign CSI-RS resources to monitor certain beams from the gNB. At this point, and assuming that the UE did not report its location, the only certain information the gNB has obtained about the channel state is the beam information associated to the Physical Random Access Channel (PRACH) sent by the UE. The gNB has no evidence on any alternative good beams, both UE and BS beams. Obtaining an alternative beam, or pair of beams, in case of multi-antenna UEs, is essential in order to maintain a good communication link between peers in an eventual disappearance of the active beamformed connection.

This section of the Thesis provides a list of strategies to create lists of pairs of beams to monitor with CSI-RS measurements to enable beam tracking. Compared to the beam tracking with SSB described in Subsection 5.2, tracking with additional CSI-RS measurements may benefit of the reduced measurement and reporting periods of CSI-RS.

\subsubsection{Beam tracking strategies}

This subsection serves to introduce the strategy alternatives to create the list of beam pairs to monitor with CSI-RS.

\section{Alternative A: Next-to-optimal beams}

This candidate beam pair strategy suggests tracking the immediate neighbouring beams of the active combination of gNB and UE beams or beam pair. The candidate beams are selected in the azimuth and elevation domains. This beam tracking strategy is inspired by the natural walk of the users. User moves continuously along its path and so does the azimuth and elevation angles of the principal multipath contribution in LoS condition. Specific angular directions belong to unique beams. As a consequence, it is expected that the user visits contiguous beam prints along its path. The beam numbering strategy matches the codeword numbering described in Section 3.3. Beam ids are divided in $v$ groups of consecutive values from 0 to the number of beams in the codebook, where $v$ is the number of elevation beams. Each elevation beam group is composed of $h$ beam ids, corresponding to beam ids within the same elevation plane.

Take Figure 5.18 as an example of the beams monitored with this beam tracking strategy. Beams are represented by circles in a matrix, where the rows correspond to different elevation beams and the columns to different azimuth 


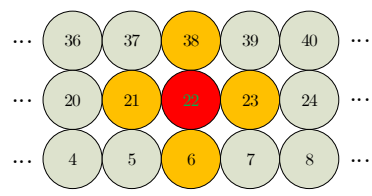

BS

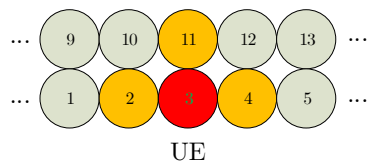

$\mathrm{UE}$

Figure 5.18: The beam tracking strategy Alternative A monitors the immediate neighbours of the current active beams.

Table 5.7: Percentage of CSI-RS overhead of a single user over the available time/frequency resources for beam tracking strategy Alternative A.

\begin{tabular}{|r|c|r|}
\hline Period & $\rho=1$ & $\rho=1 / 2$ \\
\hline \hline $10 \mathrm{~ms}$ & $0.17 \%$ & $0.09 \%$ \\
\hline $20 \mathrm{~ms}$ & $0.09 \%$ & $0.04 \%$ \\
\hline $40 \mathrm{~ms}$ & $0.04 \%$ & $0.02 \%$ \\
\hline $80 \mathrm{~ms}$ & $0.02 \%$ & $0.01 \%$ \\
\hline
\end{tabular}

beams. The red circles denote the active beams. In this example, the active gNB beam id is 22 and the active UE beam id is 3 . The yellow circles depict the neighbouring beams monitored with CSI-RS. Back to the example, the monitored neighbouring beams on the gNB side are, in ascending order, 6, 21, 23 and 38. In the same way, the monitored neighbouring beams on the UE side are 2, 4 and 11. Since the UE has two elevation beams, only one elevation beam can be considered. Note that the active beams are also monitored with CSI-RS.

Beam measurements are performed for each combination of gNB and UE beam. The number of pairs of beams monitored with CSI-RS $B$ is 20: 5 transmit beams on the gNB side and 4 receive beams on the UE side. A CSI-RS resource is allocated for each beam pair measurement. Therefore, the number of CSI-RS resources for a given user using this beam tracking strategy is $B$. The signalling overhead due to CSI-RS was calculated in Section 2.1.4 and depends on the number of beams pairs to monitor $B$ and the CSI-RS density $\rho$. Table 5.7 summarises the percentage of CSI-RS overhead over total number of resources available in the carrier for the supported CSI-RS resource allocation periods and $\rho$ values. As it can be seen, the contribution of CSI-RS is marginal with this beam tracking strategy, with a maximum overhead contribution of $0.17 \%$. 
Table 5.8: Percentage of CSI-RS overhead of a single user over the available time/frequency resources for beam tracking strategy Alternative B.

\begin{tabular}{|r|c|r|}
\hline Period & $\rho=1$ & $\rho=1 / 2$ \\
\hline \hline $10 \mathrm{~ms}$ & $0.49 \%$ & $0.24 \%$ \\
\hline $20 \mathrm{~ms}$ & $0.24 \%$ & $0.12 \%$ \\
\hline $40 \mathrm{~ms}$ & $0.12 \%$ & $0.06 \%$ \\
\hline $80 \mathrm{~ms}$ & $0.06 \%$ & $0.03 \%$ \\
\hline
\end{tabular}

\section{Alternative B: Next-to-optimal and equally separated in azimuth beams}

This candidate beam pair strategy is an extension of the Alternative A strategy. The present strategy suggests tracking additional gNB and UE beams. The additional UE beams are equally separated by 2 azimuth beams from the active UE beam. Similarly, the gNB beams are equally separated by 4 azimuth beams. This beam tracking strategy aims at detecting significant contributions in beams which are distant to the active beams. This can be the case of sudden blockage episodes due to obstacles, where an abrupt change in the Angle of Arrival (AoA) or Angle of Departure (AoD) may be experienced. Moreover, this strategy monitors 8 transmit or gNB beams with CSI-RS resources, which is the maximum supported number of transmit beams to monitor according to the NR standard.

Figure 5.19 is an example of the beams selected for beam tracking with the Alternative B strategy. The red circles denote the active beams on both the gNB and UE side. The yellow circles depict the neighbouring beams, and the blue circles represent the additional beams to monitor with CSI-RS. Like in Alternative A strategy, the active beams are also monitored with CSI-RS.

Beam measurements are performed for each combination of gNB and UE beam. So, the number of pairs of beams monitored with CSI-RS $B$ is 56: 8 transmit beams on the gNB side and 7 receive beams on the UE side. The signalling overhead due to CSI-RS is summarised in Table 5.8. Compared to the beam tracking Alternative A, the contribution of CSI-RS to the overhead is larger with the beam tracking strategy 5 , but still the percentage of resources allocated for CSI-RS is below $1 \%$ in all cases, with a maximum overhead contribution of $0.49 \%$. 


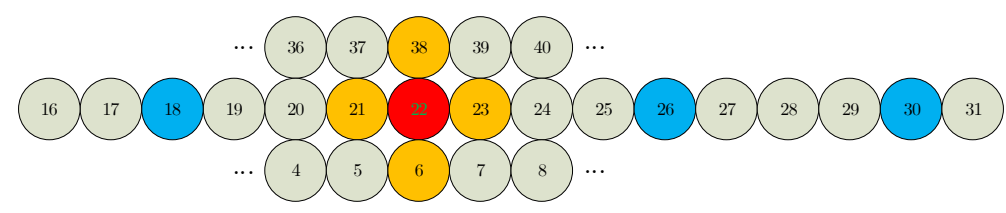

BS

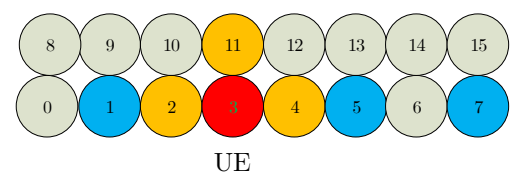

Figure 5.19: The beam tracking strategy Alternative B monitors the immediate neighbours of the current active beams and some additional directions.

\subsubsection{Urban macro scenario}

The performance analysis of the CSI-RS based beam tracking alternatives begins with the evaluation in the UMa scenario at pedestrian and vehicular speeds.

\section{Pedestrian speed}

Figure 5.20 illustrates the evolution of the gNB and UE beam ids during the walk at pedestrian speed. Assuming the largest SS burst period value of $160 \mathrm{~ms}$, the figure represents the evolution of the gNB and UE beams throughout the user walk for different CSI-RS periods. The baseline case is also represented to serve as a visual reference of the beam tracking ability.

As it can be seen in the figure, and regardless of the value of the CSI-RS period, beam updates practically occur simultaneously to the baseline case, which has a complete channel knowledge every $1 \mathrm{~ms}$. The addition of CSI-RS to beam tracking represents a substantial gain compared to the SSB-only monitoring strategy for the longest SS burst period illustrated in Figure 5.8. Definitely, periodic CSI-RS beam measurements may contribute reducing beam update delays with respect to SSB beam tracking.

Figure 5.21 illustrates the evolution of the SINR along the UE walk with different SS burst period and a fixed CSI-RS period. The selected CSI-RS period value for this analysis is $10 \mathrm{~ms}$ in order to provide the possible fastest beam updates with CSI-RS resources. As it can be seen, beam tracking with CSI-RS 

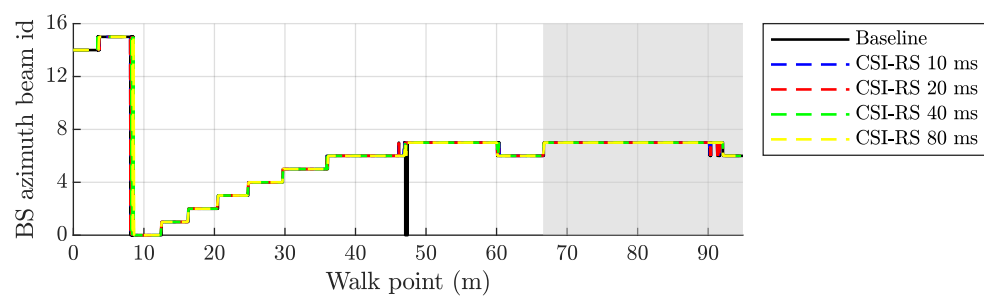

(a) gNB azimuth beam id.
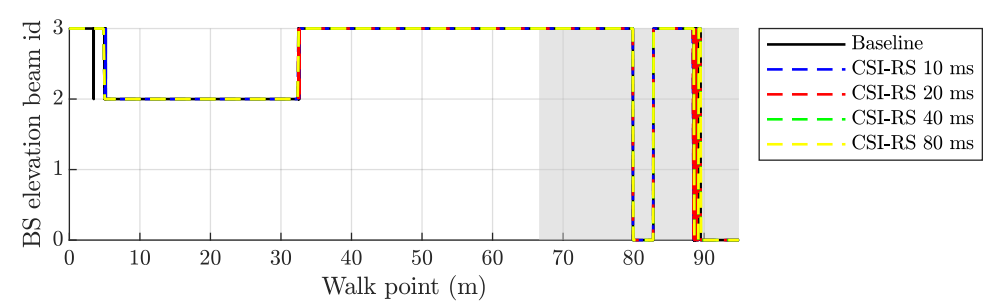

(b) gNB elevation beam id.
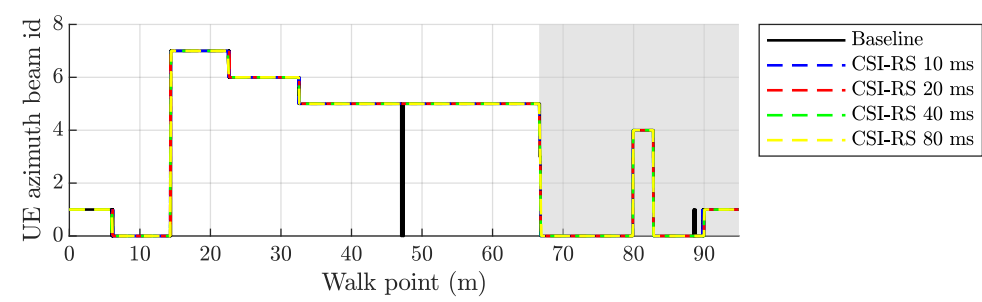

(c) UE azimuth beam id.
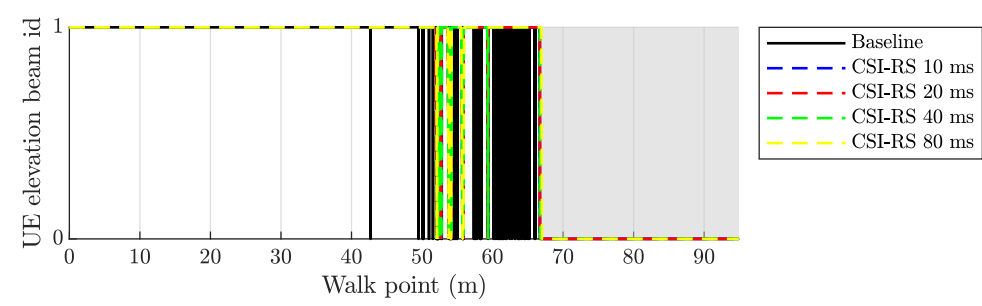

(d) UE elevation beam id.

Figure 5.20: Beam ids changes with short SS burst period and CSI-RS beam tracking in UMa walk at pedestrian speed. The greyed area corresponds to NLoS region. 


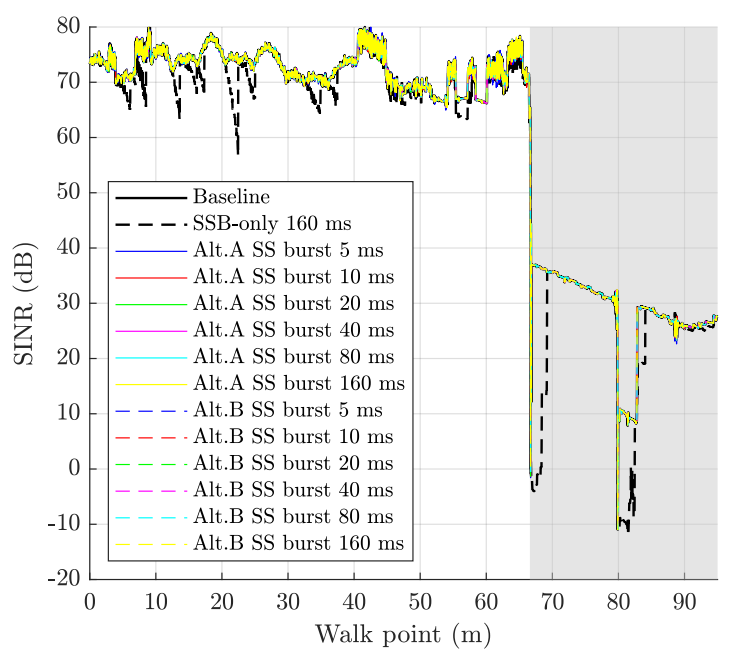

Figure 5.21: SINR evolution in the UMa walk at pedestrian speed for the supported SS burst periods and a fixed CSI-RS value of $10 \mathrm{~ms}$.

provides larger SINR values than SSB-only tracking due to the improved beam alignment, and the performance is practically identical to the baseline case.

Good SINR values may lead to similarly good throughput values as illustrated in Figure 5.22. The maximum achievable data rate will depend on the signalling overhead, which finds in SSB transmissions its major contributor. As expected, the peak data rate is larger as the SS burst period increases. Therefore, enlarging SS burst period is desirable as long as the beam tracking ability is not compromised.

Note that the throughput curves experience a small drop after the transition to NLoS but the stationary rate is quickly recovered and maintained until the walk point 80 metres, when the useful signal experiences a profound power drop. In comparison with the SSB beam sweeping strategy represented with the dashed black curve, the faster beam updates obtained from CSI-RS measurement reports for any of the two beam tracking strategies reduces the significant rate drops experienced with the SSB beam sweeping strategy in NLoS.

The analysis continues with the study of the influence of the CSI-RS period value assuming that the SS burst period is fixed. Figure 5.23 serves to illustrate the evolution in the UMa walk of the SINR level for Alternative A and Alternative B CSI-RS strategies with different CSI-RS periods for the longest 


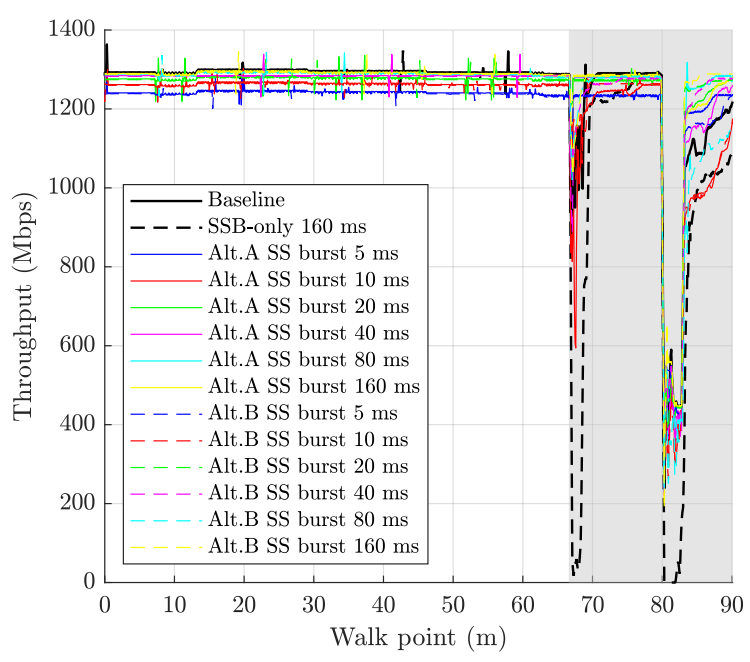

Figure 5.22: User throughput evolution in the UMa walk at pedestrian speed for the supported SS burst periods and a fixed CSI-RS value of $10 \mathrm{~ms}$.

SS burst period. The figure also plots the same evolution for the baseline case and SSB beam sweeping. As it can be seen, CSI-RS drastically reduces the SINR drops obtained with the SSB beam sweeping. Practically all the CSI-RS combinations provides the same beam tracking performance in terms of SINR level. As a result, all the CSI-RS curves are superimposed with the baseline case.

Figure 5.24 illustrates the evolution in the UMa walk of the user throughput for the baseline case and the two CSI-RS beam tracking strategies with the longest SS burst configuration and different CSI-RS period values. As it can be seen, all the plotted configurations practically provide the same data rate trends, with little differences in NLoS and the largest CSI-RS value of $80 \mathrm{~ms}$.

Table 5.9 summarises the average throughput experienced in the UMa walk at pedestrian speed for Alternative A (Alt.A), Alternative B (Alt.B), baseline and SSB-only beam tracking. Using CSI-RS resources for beam management outperforms the average throughput experienced in the whole UE walk. The only exception is found at the LoS region, where simply performing beam sweeping of SSB provides similar throughput values to the CSI-RS-based beam tracking strategies. It can be derived that the signalling overhead due to CSI-RS has no impact on the top data rate. This confirms the theoretical analysis of the CSI-RS overhead, which percentages over the available amount 


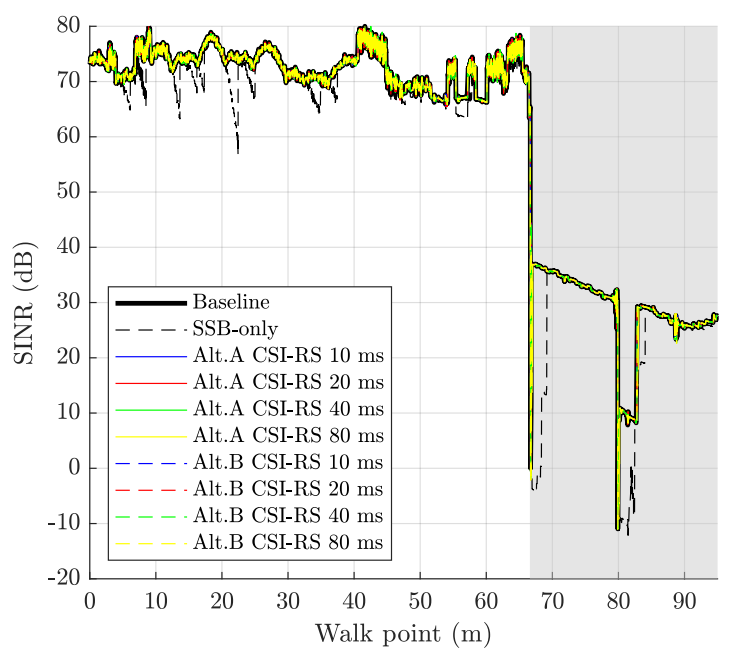

Figure 5.23: SINR evolution in the UMa walk at pedestrian speed with long SS burst period.

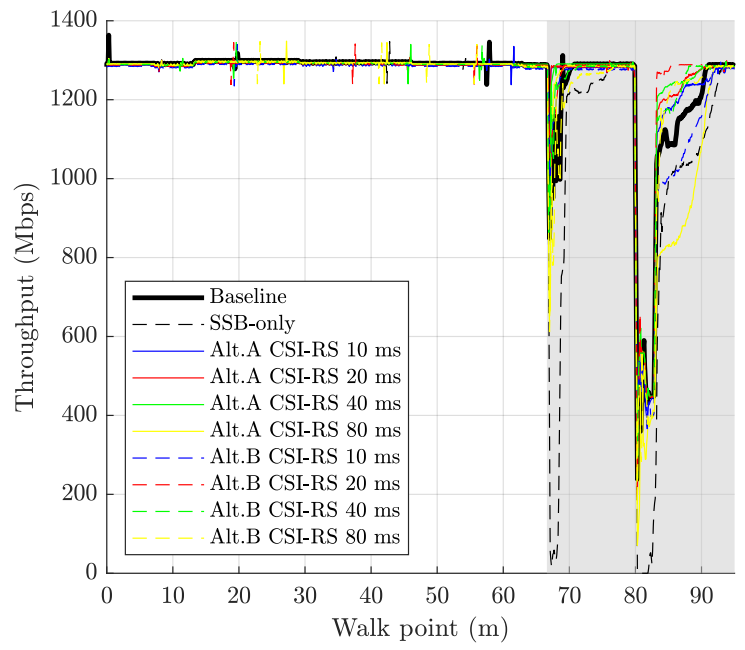

Figure 5.24: User throughput evolution in the UMa walk at pedestrian speed with long SS burst period. 
Table 5.9: Average throughput values at pedestrian speed for baseline and beam tracking strategies with different SS burst and CSI-RS periods.

\begin{tabular}{|c|c|c|c|c|c|}
\hline \multirow{2}{*}{ Strategy } & \multirow{2}{*}{$\begin{array}{c}\text { SS burst } \\
\text { period (ms) }\end{array}$} & \multirow{2}{*}{$\begin{array}{c}\text { CSI-RS } \\
\text { period }(\mathrm{ms})\end{array}$} & \multicolumn{3}{|c|}{ Avg. Throughput (Mbps) } \\
\hline & & & All & LoS & NLoS \\
\hline Baseline & - & - & 1243.38 & 1294.29 & 1080.69 \\
\hline \multirow{2}{*}{ SSB-only } & 20 & - & 1220.08 & 1278.61 & 1033.07 \\
\hline & 160 & - & 1203.72 & 1292.73 & 919.31 \\
\hline \multirow{8}{*}{ Alt.A } & \multirow{4}{*}{20} & 10 & 1237.40 & 1279.50 & 1102.87 \\
\hline & & 20 & 1234.61 & 1278.07 & 1095.74 \\
\hline & & 40 & 1233.52 & 1278.10 & 1091.08 \\
\hline & & 80 & 1234.91 & 1278.45 & 1095.78 \\
\hline & \multirow{4}{*}{160} & 10 & 1242.14 & 1290.10 & 1088.92 \\
\hline & & 20 & 1246.03 & 1291.40 & 1101.07 \\
\hline & & 40 & 1247.57 & 1292.11 & 1105.23 \\
\hline & & 80 & 1240.35 & 1292.37 & 1074.14 \\
\hline \multirow{8}{*}{ Alt.B } & \multirow{4}{*}{20} & 10 & 1232.20 & 1275.51 & 1093.81 \\
\hline & & 20 & 1175.89 & 1275.68 & 916.19 \\
\hline & & 40 & 1234.81 & 1277.15 & 1099.49 \\
\hline & & 80 & 1233.89 & 1278.02 & 1092.88 \\
\hline & \multirow{4}{*}{160} & 10 & 1242.49 & 1286.14 & 1103.04 \\
\hline & & 20 & 1246.47 & 1289.42 & 1109.25 \\
\hline & & 40 & 1242.87 & 1291.10 & 1088.74 \\
\hline & & 80 & 1243.77 & 1291.88 & 1090.04 \\
\hline
\end{tabular}

of resource are summarised in Table 5.7 for Alternative A and Table 5.8 for Alternative B.

The advantage of beam tracking with CSI-RS over SSB is found in NLoS for the longest SS burst period of $160 \mathrm{~ms}$.

Table 5.10 lists the beam alignment ratio and BGL for each beam tracking configuration in consideration. As occurred with the throughput, beam tracking figures are similar to the baseline case for the SSB and CSI-RS beam tracking strategies for an SS burst period of $20 \mathrm{~ms}$. As it can be seen, using CSI-RS beam measurements with the longest SS burst period helps achieving the same beam alignment ratio of the baseline case. 
Table 5.10: Beam alignment ratio and BGL at pedestrian speed for baseline and the beam tracking alternatives configured with multiple SS burst and

CSI-RS periods.

\begin{tabular}{|c|c|c|c|c|c|c|}
\hline \multirow{2}{*}{ Strategy } & \multirow{2}{*}{$\begin{array}{c}\text { SS burst } \\
\text { period }(\mathrm{ms})\end{array}$} & \multirow{2}{*}{$\begin{array}{c}\text { CSI-RS } \\
\text { period }(\mathrm{ms})\end{array}$} & \multicolumn{3}{|c|}{$\mathbf{R}_{\text {alignment }}$} & \multirow{2}{*}{$\begin{array}{l}\text { BGL } \\
(\mathrm{dB})\end{array}$} \\
\hline & & & Pair & gNB & $\mathbf{U E}$ & \\
\hline Baseline & - & - & 1.00 & 1.00 & 1.00 & 0.00 \\
\hline \multirow{2}{*}{ SSB-only } & 20 & - & 0.90 & 0.96 & 0.93 & 2.58 \\
\hline & 160 & - & 0.62 & 0.81 & 0.74 & 4.78 \\
\hline \multirow{8}{*}{ Alt.A } & \multirow{4}{*}{20} & 10 & 0.98 & 0.99 & 0.99 & 2.19 \\
\hline & & 20 & 0.97 & 0.99 & 0.98 & 2.47 \\
\hline & & 40 & 0.97 & 0.99 & 0.98 & 2.41 \\
\hline & & 80 & 0.96 & 0.98 & 0.97 & 2.45 \\
\hline & \multirow{4}{*}{160} & 10 & 0.98 & 0.99 & 0.99 & 2.19 \\
\hline & & 20 & 0.97 & 0.99 & 0.98 & 2.45 \\
\hline & & 40 & 0.97 & 0.99 & 0.98 & 3.37 \\
\hline & & 80 & 0.96 & 0.98 & 0.97 & 4.69 \\
\hline \multirow{8}{*}{ Alt.B } & \multirow{4}{*}{20} & 10 & 0.98 & 0.99 & 0.99 & 1.36 \\
\hline & & 20 & 0.98 & 0.99 & 0.99 & 1.53 \\
\hline & & 40 & 0.97 & 0.99 & 0.98 & 1.79 \\
\hline & & 80 & 0.96 & 0.98 & 0.97 & 2.04 \\
\hline & \multirow{4}{*}{160} & 10 & 0.98 & 0.99 & 0.99 & 1.35 \\
\hline & & 20 & 0.98 & 0.99 & 0.99 & 1.52 \\
\hline & & 40 & 0.97 & 0.99 & 0.98 & 2.03 \\
\hline & & 80 & 0.97 & 0.98 & 0.98 & 3.10 \\
\hline
\end{tabular}




\section{CHAPTER 5. BEAM TRACKING TECHNIQUES}

\section{Vehicular speed}

Figure 5.25 illustrates the evolution of the gNB and UE beam ids during the walk at pedestrian speed. The figure represents the baseline case, Alternative A and Alternative B.

The situation in the pedestrian speed is repeated in the vehicular case. The use of CSI-RS resources for beam tracking helps reduce the beam update delay obtained with tracking SSBs due to the more frequent updates of relevant beams.

Figure 5.26 illustrates the evolution of the SINR along the walk for the baseline, SSB beam tracking, Alternative A. and Alternative B. The later two options are evaluated with different CSI-RS periods. In this figure the SS burst period is set to $160 \mathrm{~ms}$. As it can be seen, CSI-RS periods of $40 \mathrm{~ms}$ and $80 \mathrm{~ms}$ experience longer beam misalignment periods and, consequently, their SINR presents stronger fluctuations. This signal fading is motivated by the combination of delayed updates and velocity, which leads the user to cover large distances in a misalignment situation.

Figure 5.27 illustrates the evolution of the experienced throughput for the beam tracking strategies in the previous SINR analysis. The throughput in LoS is the same for all the CSI-RS periods. However, the throughput experiences a significant degradation in the NLoS transition. More precisely, in the walk points 67 metres and 80 metres. The longer the CSI-RS period, the larger the throughput reduction is. This is, again, an effect of the beam misalignment period. For this particular scenario and, the CSI-RS values of $10 \mathrm{~ms}$ and $20 \mathrm{~ms}$ are recommended as they provide the best data rate during the whole walk.

Table 5.11 summarises the average throughput for the UMa walk at vehicular speed for the baseline case, and beam tracking strategies with SSB and CSI-RS. In light of the results, the CSI-RS beam tracking strategies outperform beamsweeping SSBs in terms of average throughput. There is little room for improvement in LoS region for CSI-RS, and simply performing beam sweeping of SSBs is enough to provide good data rate at vehicular speeds. However, NLoS situations demand beam CSI-RS-based tracking strategies with faster beam updates. And the update velocity is more critical than in the pedestrian speed case. According to the results, the CSI-RS period should not be longer than $20 \mathrm{~ms}$ for any SS burst period.

The second part of the beam tracking analysis is the beam tracking ability. Table 5.12 summarises the beam alignment ratio and BGL value for each beam tracking configuration in consideration for the vehicular mobility in UMa for two SS burst periods. In general, the overall beam alignment ratio is influenced by the fast UE elevation beam fluctuations, which are hard to follow. As the CSI-RS periodicity increases, the beam alignment ratio decreases, with 


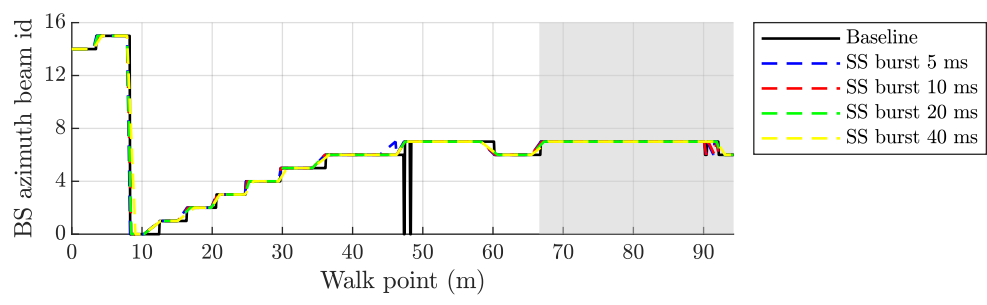

(a) gNB azimuth beam id.

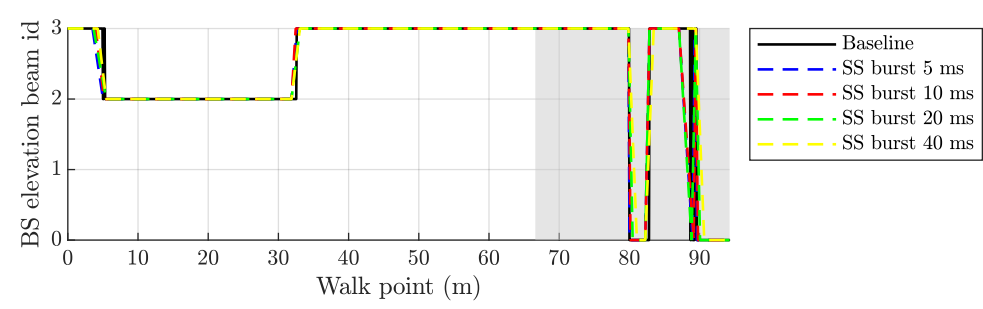

(b) gNB elevation beam id.

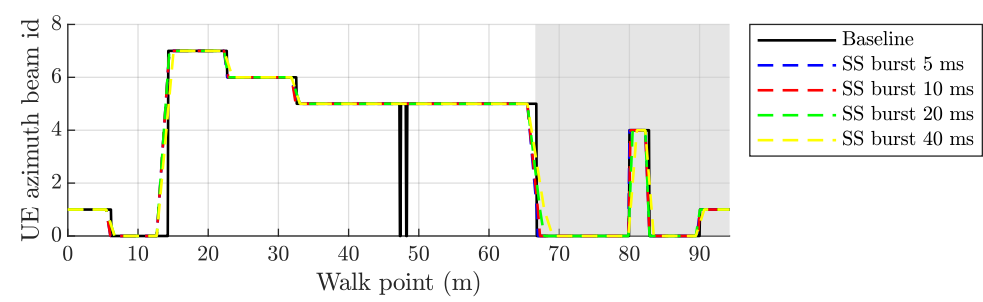

(c) UE azimuth beam id.

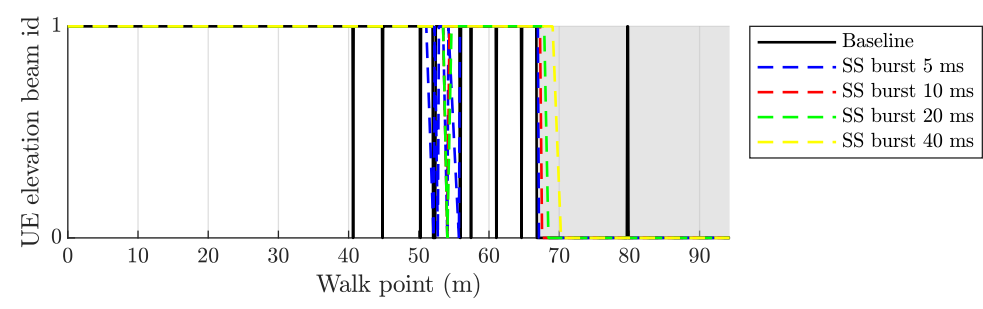

(d) UE elevation beam id.

Figure 5.25: Beam ids changes with long SS burst period and CSI-RS beam tracking in UMa walk at pedestrian speed. The greyed area corresponds to NLoS region. 


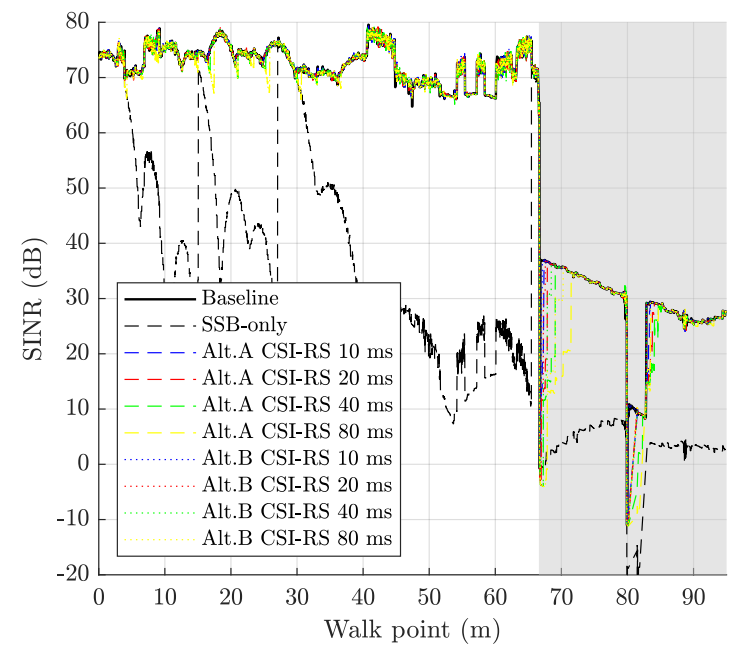

Figure 5.26: SINR evolution in time at vehicular speed and long SS burst period in UMa scenario.

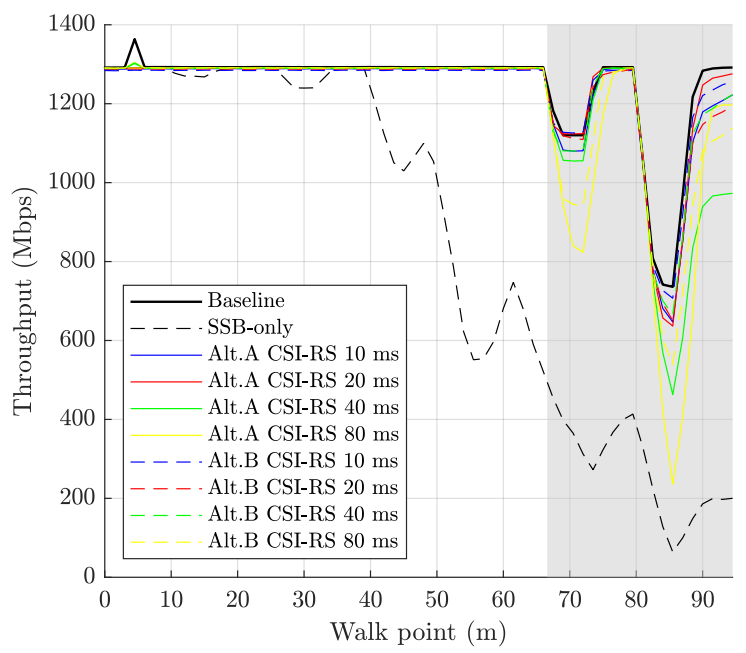

Figure 5.27: User throughput evolution in time at vehicular speed and long SS burst period in UMa scenario. 
Table 5.11: Average throughput values obtained at vehicular speed for the baseline case and for the beam tracking strategies with different SS burst and CSI-RS periods.

\begin{tabular}{|c|c|c|c|c|c|}
\hline \multirow{2}{*}{ Strategy } & \multirow{2}{*}{$\begin{array}{c}\text { SS burst } \\
\text { period }(\mathrm{ms})\end{array}$} & \multirow{2}{*}{$\begin{array}{c}\text { CSI-RS } \\
\text { period }(\mathrm{ms})\end{array}$} & \multicolumn{3}{|c|}{ Avg. Throughput (Mbps) } \\
\hline & & & All & LoS & NLoS \\
\hline Baseline & - & - & 1242.54 & 1294.10 & 1120.41 \\
\hline \multirow{2}{*}{ SSB-only } & 20 & 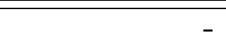 & 1115.02 & 1269.45 & $\overline{7749.26}$ \\
\hline & 160 & - & 852.05 & 1098.85 & 267.52 \\
\hline \multirow{8}{*}{ Alt.A } & \multirow{4}{*}{20} & 10 & 1220.22 & 1278.10 & 1083.14 \\
\hline & & 20 & 1172.03 & 1276.11 & 925.54 \\
\hline & & 40 & 1187.94 & 1276.13 & 979.07 \\
\hline & & 80 & 1198.43 & 1276.46 & 1013.63 \\
\hline & \multirow{4}{*}{160} & 10 & 1229.14 & 1288.41 & 1088.76 \\
\hline & & 20 & 1236.91 & 1289.78 & 1111.71 \\
\hline & & 40 & 1201.33 & 1290.64 & 989.79 \\
\hline & & 80 & 1187.50 & 1291.04 & 942.28 \\
\hline \multirow{8}{*}{ Alt.B } & \multirow{4}{*}{20} & 10 & 1227.95 & 1274.17 & 1118.48 \\
\hline & & 20 & 1141.53 & 1274.17 & 827.37 \\
\hline & & 40 & 1165.71 & 1275.20 & 906.39 \\
\hline & & 80 & 1200.39 & 1276.02 & 1021.26 \\
\hline & \multirow{4}{*}{160} & 10 & 1235.13 & 1284.50 & 1118.22 \\
\hline & & 20 & 1228.43 & 1287.81 & 1087.80 \\
\hline & & 40 & 1230.92 & 1289.74 & 1091.61 \\
\hline & & 80 & 1208.53 & 1290.27 & 1014.95 \\
\hline
\end{tabular}


Table 5.12: Beam alignment ratio and BGL in the UMa scenario at vehicular speed for the baseline case and the beam tracking alternatives with multiple

SS burst and CSI-RS periods.

\begin{tabular}{|c|c|c|c|c|c|c|}
\hline \multirow{2}{*}{ Strategy } & \multirow{2}{*}{$\begin{array}{c}\text { SS burst } \\
\text { period }(\mathrm{ms})\end{array}$} & \multirow{2}{*}{$\begin{array}{c}\text { CSI-RS } \\
\text { period }(\mathrm{ms})\end{array}$} & \multicolumn{3}{|c|}{$\mathbf{R}_{\text {alignment }}$} & \multirow{2}{*}{$\begin{array}{l}\text { BGL } \\
(\mathrm{dB})\end{array}$} \\
\hline & & & Pair & gNB & $\mathbf{U E}$ & \\
\hline Baseline & - & - & 1.00 & 1.00 & 1.00 & 0.00 \\
\hline \multirow{2}{*}{ SSB-only } & 20 & - & 0.61 & 0.74 & 0.72 & 5.53 \\
\hline & 160 & - & 0.22 & 0.38 & 0.26 & 14.73 \\
\hline \multirow{8}{*}{ Alt.A } & \multirow{4}{*}{20} & 10 & 0.97 & 0.99 & 0.98 & 6.53 \\
\hline & & 20 & 0.94 & 0.98 & 0.96 & 6.91 \\
\hline & & 40 & 0.91 & 0.96 & 0.94 & 6.39 \\
\hline & & 80 & 0.83 & 0.92 & 0.88 & 6.24 \\
\hline & \multirow{4}{*}{160} & 10 & 0.97 & 0.99 & 0.98 & 6.62 \\
\hline & & 20 & 0.94 & 0.98 & 0.96 & 7.49 \\
\hline & & 40 & 0.89 & 0.95 & 0.92 & 8.45 \\
\hline & & 80 & 0.82 & 0.91 & 0.88 & 9.26 \\
\hline \multirow{8}{*}{ Alt.B } & \multirow{4}{*}{20} & 10 & 0.98 & 0.99 & 0.98 & 3.56 \\
\hline & & 20 & 0.96 & 0.98 & 0.97 & 4.42 \\
\hline & & 40 & 0.93 & 0.96 & 0.96 & 5.13 \\
\hline & & 80 & 0.84 & 0.92 & 0.90 & 5.85 \\
\hline & \multirow{4}{*}{160} & 10 & 0.98 & 0.99 & 0.98 & 3.68 \\
\hline & & 20 & 0.96 & 0.98 & 0.97 & 5.17 \\
\hline & & 40 & 0.92 & 0.95 & 0.96 & 6.41 \\
\hline & & 80 & 0.85 & 0.91 & 0.91 & 7.36 \\
\hline
\end{tabular}

a maximum registered performance loss of $12 \%$ and $10 \%$ for beam tracking Alternative A and B respectively. Beam tracking Alternative B provides better beam alignment that Alternative A since the extra monitoring beams help to detect the next beam pair faster. With respect to the BGL, the Alternative $B$ provides smaller values than Alternative A. However, the BGL value is a bit high due to the above-mentioned rapid UE beam changes in the elevation plane.

\subsubsection{Urban micro scenario}

This part of the section evaluates the performance of the beam tracking alternatives in the UMi scenario at pedestrian and vehicular speeds. 


\section{Pedestrian speed}

Figure 5.28 illustrates the evolution of the gNB and UE beam ids along the user walk at pedestrian speed. Assuming the largest SS burst period value of $160 \mathrm{~ms}$, the figure represents the evolution of the gNB and UE beams throughout the user walk for different CSI-RS periodicities. The baseline case is also represented to serve as a visual reference of the beam tracking ability. Despite of the large SS burst period, the more frequent CSI-RS beam measurements provide an excellent beam tracking.

The use of periodic CSI-RS resources for beam tracking clearly reduces the beam update delay with respect to SSB beam tracking. The beam updates with any of the CSI-RS strategies depicted in Figure 5.28 practically occur simultaneously to the baseline case.

Figure 5.29 serves to illustrate the evolution in the UMi walk of the SINR level in $\mathrm{dB}$ for Alternative A and Alternative B beam tracking strategies with the longest SS burst period and different CSI-RS periods. The figure also plots the same evolution for the baseline case and SSB beam sweeping. As it can be seen, CSI-RS eliminates the SINR fluctuations obtained with the SSB beam sweeping in the initial 30 metres. In addition, all the CSI-RS combinations provides the same SINR level. As a result, all the CSI-RS curves are superimposed with the baseline case.

Figure 5.30 illustrates the evolution in the UMi walk of the user throughput for the baseline case and the two CSI-RS beam tracking strategies with the longest SS burst configuration and different CSI-RS period values. As it can be seen, all the plotted configurations practically provide the same data rate trends, with little differences in NLoS and the largest CSI-RS value of $80 \mathrm{~ms}$. Note that the throughput curves experience a small drop after the transition to NLoS but the stationary rate is quickly recovered and maintained until point 85 metres, when the rays arriving at the UE experience a profound SINR drop to $10 \mathrm{~dB}$. In comparison with the SSB beam sweeping strategy represented with the dashed black curve, the faster beam updates obtained from CSI-RS measurement reports for any of the two beam tracking strategies reduces the significant rate drops experienced with the SSB beam sweeping strategy in NLoS.

Table 5.13 summarises the average throughput for the two beam tracking alternatives using CSI-RS beam measurements for the UE walk in the UMi scenario at pedestrian speed. Using CSI-RS resources for beam management outperforms the average throughput experienced in the SSB-only beamsweeping. The only exception is found at the LoS region, where simply performing beam sweeping of SSB provides good tracking at pedestrian speed. As a result, the average throughput in the LoS region is practically the same for all the 


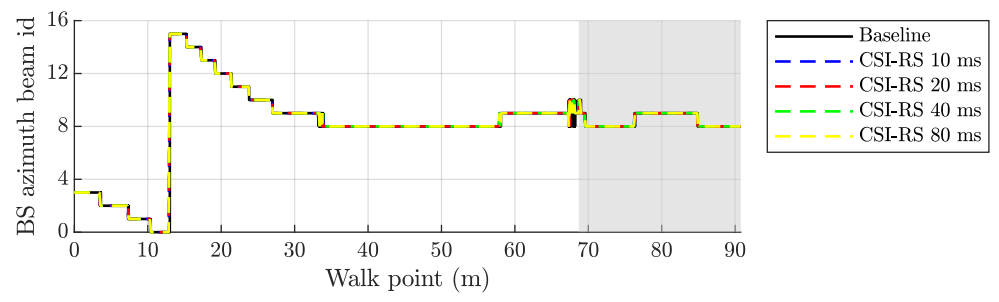

(a) gNB azimuth beam id.
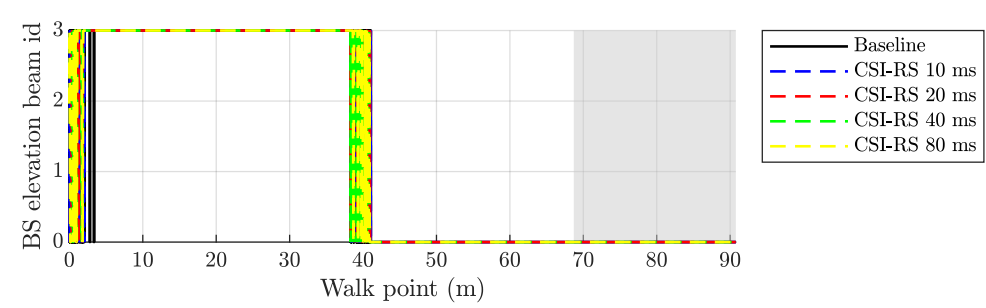

(b) gNB elevation beam id.

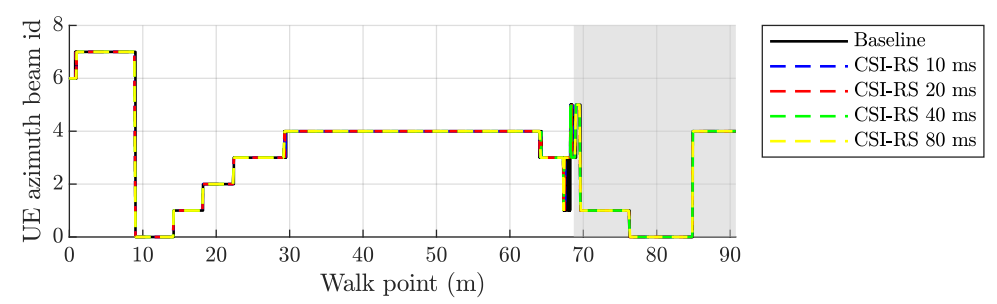

(c) UE azimuth beam id.

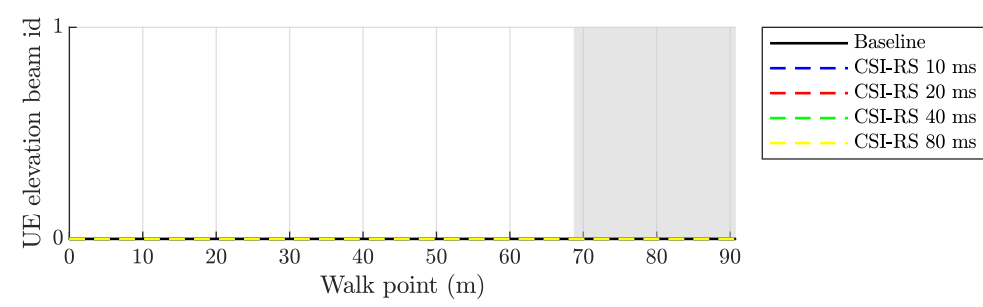

(d) UE elevation beam id.

Figure 5.28: Beam ids changes with long SS burst period and CSI-RS beam tracking in UMi walk at pedestrian speed. The greyed area corresponds to NLoS region. 


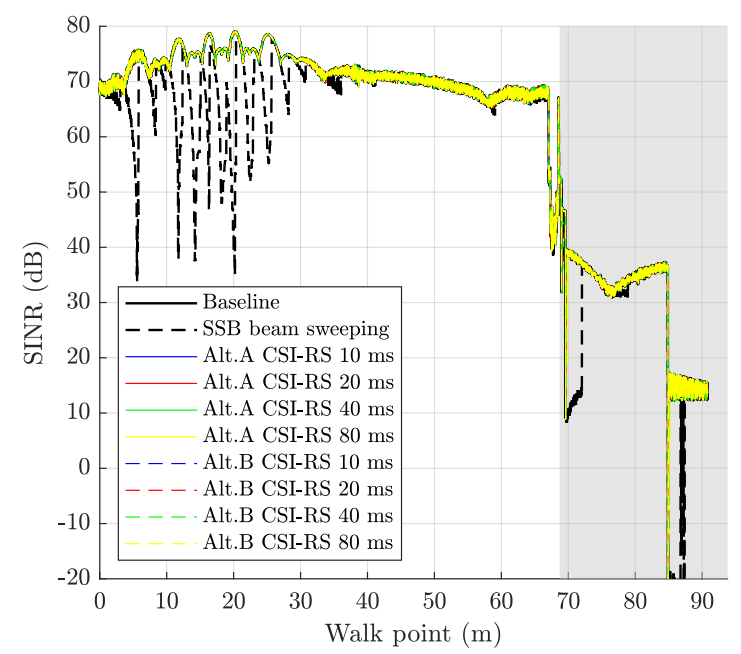

Figure 5.29: SINR evolution in the UMi walk at pedestrian speed with long SS burst period.

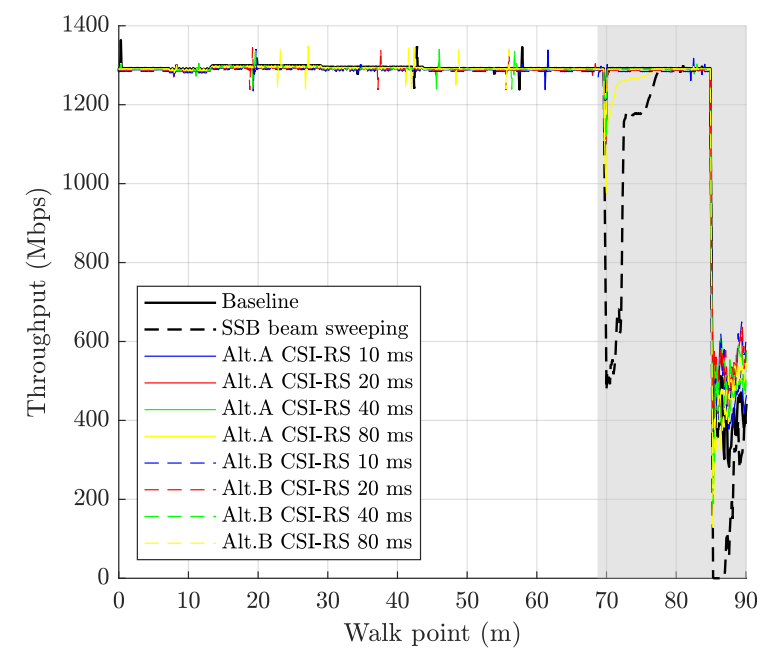

Figure 5.30: User throughput evolution in the UMi walk at pedestrian speed with long SS burst period. 
Table 5.13: Average throughput values at pedestrian speed for baseline and beam tracking strategies with different SS burst and CSI-RS periods.

\begin{tabular}{|c|c|c|c|c|c|}
\hline \multirow{2}{*}{ Strategy } & \multirow{2}{*}{$\begin{array}{c}\text { SS burst } \\
\text { period (ms) }\end{array}$} & \multirow{2}{*}{$\begin{array}{c}\text { CSI-RS } \\
\text { period }(\mathrm{ms})\end{array}$} & \multicolumn{3}{|c|}{ Avg. Throughput (Mbps) } \\
\hline & & & All & LoS & NLoS \\
\hline Baseline & - & - & 1243.38 & 1294.29 & 1080.69 \\
\hline \multirow{2}{*}{ SSB-only } & 20 & - & 1220.08 & 1278.61 & 1033.07 \\
\hline & 160 & - & 1203.72 & 1292.73 & 919.31 \\
\hline \multirow{8}{*}{ Alt.A } & \multirow{4}{*}{20} & 10 & 1237.40 & 1279.50 & 1102.87 \\
\hline & & 20 & 1234.61 & 1278.07 & 1095.74 \\
\hline & & 40 & 1233.52 & 1278.10 & 1091.08 \\
\hline & & 80 & 1234.91 & 1278.45 & 1095.78 \\
\hline & \multirow{4}{*}{160} & 10 & 1242.14 & 1290.10 & 1088.92 \\
\hline & & 20 & 1246.03 & 1291.40 & 1101.07 \\
\hline & & 40 & 1247.57 & 1292.11 & 1105.23 \\
\hline & & 80 & 1240.35 & 1292.37 & 1074.14 \\
\hline \multirow{8}{*}{ Alt.B } & \multirow{4}{*}{20} & 10 & 1232.20 & 1275.51 & 1093.81 \\
\hline & & 20 & 1175.89 & 1275.68 & 916.19 \\
\hline & & 40 & 1234.81 & 1277.15 & 1099.49 \\
\hline & & 80 & 1233.89 & 1278.02 & 1092.88 \\
\hline & \multirow{4}{*}{160} & 10 & 1242.49 & 1286.14 & 1103.04 \\
\hline & & 20 & 1246.47 & 1289.42 & 1109.25 \\
\hline & & 40 & 1242.87 & 1291.10 & 1088.74 \\
\hline & & 80 & 1243.77 & 1291.88 & 1090.04 \\
\hline
\end{tabular}

beam tracking strategies, including SSB beam sweeping. It is worth mentioning that the small number of Resource Block (RB)s occupied by CSI-RS has a negligible impact on the throughput.

Throughput differences between beam tracking configurations are found larger in NLoS than in LoS, but still differences are not substantial enough to decide one configuration over the other for the current scenario at pedestrian speed.

Table 5.14 lists the beam alignment ratio and BGL for each beam tracking configuration in consideration. The best beam alignment ratio is achieved with the shortest CSI-RS period for both beam tracking alternatives and for any SS burst period. However, at this speed, the difference among CSI-RS periods is practically negligible. The good beam alignment ratio provides very low BGL values, with values over a hundredth of a $\mathrm{dB}$. 
Table 5.14: Beam alignment ratio and BGL at pedestrian speed for baseline and the beam tracking alternatives configured with multiple SS burst and

CSI-RS periods.

\begin{tabular}{|c|c|c|c|c|c|c|}
\hline \multirow{2}{*}{ Strategy } & \multirow{2}{*}{$\begin{array}{c}\text { SS burst } \\
\text { period (ms) }\end{array}$} & \multirow{2}{*}{$\begin{array}{c}\text { CSI-RS } \\
\text { period }(\mathrm{ms})\end{array}$} & \multicolumn{3}{|c|}{$\mathbf{R}_{\text {alignment }}$} & \multirow{2}{*}{$\begin{array}{l}\text { BGL } \\
\text { (dB) }\end{array}$} \\
\hline & & & Pair & gNB & UE & \\
\hline Baseline & - & - & 1.00 & 1.00 & 1.00 & 0.00 \\
\hline \multirow{2}{*}{ SSB-only } & 20 & - & 0.89 & 0.92 & 0.96 & 2.00 \\
\hline & 160 & - & 0.62 & 0.70 & 0.81 & 4.34 \\
\hline \multirow{8}{*}{ Alt.A } & \multirow{4}{*}{20} & 10 & 0.96 & 0.96 & 0.99 & 2.47 \\
\hline & & 20 & 0.95 & 0.96 & 0.99 & 2.18 \\
\hline & & 40 & 0.95 & 0.96 & 0.99 & 2.55 \\
\hline & & 80 & 0.94 & 0.95 & 0.98 & 2.69 \\
\hline & \multirow{4}{*}{160} & 10 & 0.96 & 0.96 & 0.99 & 2.45 \\
\hline & & 20 & 0.95 & 0.96 & 0.99 & 2.15 \\
\hline & & 40 & 0.95 & 0.96 & 0.99 & 2.59 \\
\hline & & 80 & 0.94 & 0.95 & 0.99 & 3.12 \\
\hline \multirow{8}{*}{ Alt.B } & \multirow{4}{*}{20} & 10 & 0.96 & 0.96 & 1.00 & 1.80 \\
\hline & & 20 & 0.96 & 0.96 & 1.00 & 1.76 \\
\hline & & 40 & 0.95 & 0.96 & 0.99 & 1.86 \\
\hline & & 80 & 0.95 & 0.95 & 0.99 & 1.91 \\
\hline & \multirow{4}{*}{160} & 10 & 0.96 & 0.96 & 1.00 & 1.71 \\
\hline & & 20 & 0.96 & 0.96 & 1.00 & 1.70 \\
\hline & & 40 & 0.95 & 0.96 & 0.99 & 1.86 \\
\hline & & 80 & 0.95 & 0.95 & 0.99 & 1.93 \\
\hline
\end{tabular}




\section{CHAPTER 5. BEAM TRACKING TECHNIQUES}

\section{Vehicular speed}

Figure 5.31 illustrates the evolution of the gNB and UE beam ids during the walk at vehicular speed, again assuming the longest SS burst period and multiple CSI-RS periods. The baseline case is also plotted to provide a visual reference to the lowest beam tracking performance, in contrast to the top performance provided by the baseline.

Despite the fact that CSI-RS beam tracking alternatives provide more frequent updates than SSB beamsweeping, it cannot completely eliminate the beam update delay. Due to the superior speed of the vehicular speed case, the user travels several metres in misalignment from the optimal pair of beams. And the longer the periods of beam misalignment, the longer the time the SINR is reduced. This fact can be appreciated in Figure 5.32 which illustrates the evolution of SINR level of baseline, Alt.A and Alt.B strategies for the longest SS burst period and the supported CSI-RS periods. As occurred in the pedestrian case, the SINR curves are practically identical with significant SINR level reductions in the transitions to LoS to NLoS and to the zone of ray contributions with more than 2 reflections. This signal fading is due to the combination of beam misalignment and speed.

Figure 5.33 illustrates the throughput evolution for the baseline case and the two CSI-RS beam tracking strategies with the longest SS burst configuration and different CSI-RS period values. As it can be seen, beam misalignments in NLoS due to longer CSI-RS values reduces the throughput at the "heap" starting at point 70 metres. Nevertheless, the throughput obtained in that area is practically the same as the baseline case but for the configurations with $40 \mathrm{~ms}$ and $80 \mathrm{~ms}$ of CSI-RS periodicity.

Table 5.15 summarises the average throughput for the two beam tracking alternatives using CSI-RS beam measurements for the UE walk in the UMi scenario at vehicular speed. Allocating CSI-RS resources for beam management outperforms the average throughput experienced in the whole UE walk with SSB beam sweeping. The only exception is found at the LoS region, where simply performing beam sweeping of SSB is enough to provide good tracking also at vehicular speeds. However, NLoS situations demand beam tracking strategies with CSI-RS and it is more critical than in the pedestrian speed case.

Assuming an SS burst period of $20 \mathrm{~ms}$, Alt.A provides better throughput values in average than Alt.B due to the reduced CSI-RS overhead. The best performance with Alt.A and Alt.B in terms of throughput is achieved with a CSI-RS periodicity of $10 \mathrm{~ms}$.

Assuming an SS burst period of $160 \mathrm{~ms}$, Alt.A and Alt.B provide similar average throughput values with CSI-RS periodicities of $10 \mathrm{~ms}, 20 \mathrm{~ms}$ and $40 \mathrm{~ms}$. 


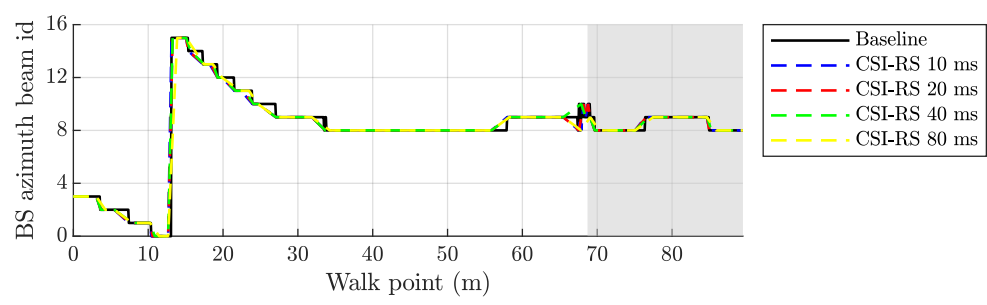

(a) gNB azimuth beam id.

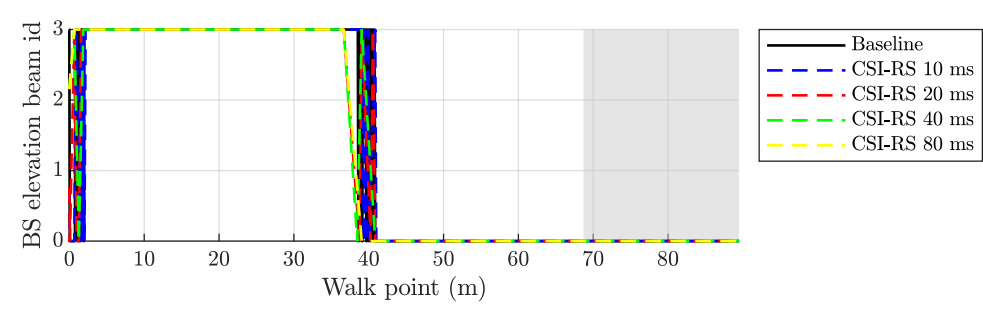

(b) gNB elevation beam id.

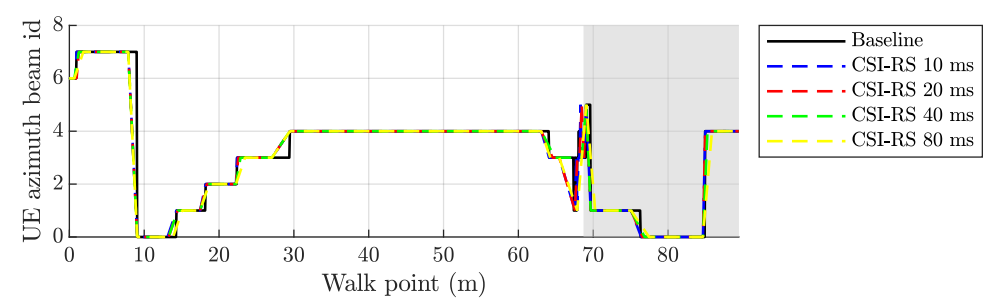

(c) UE azimuth beam id.

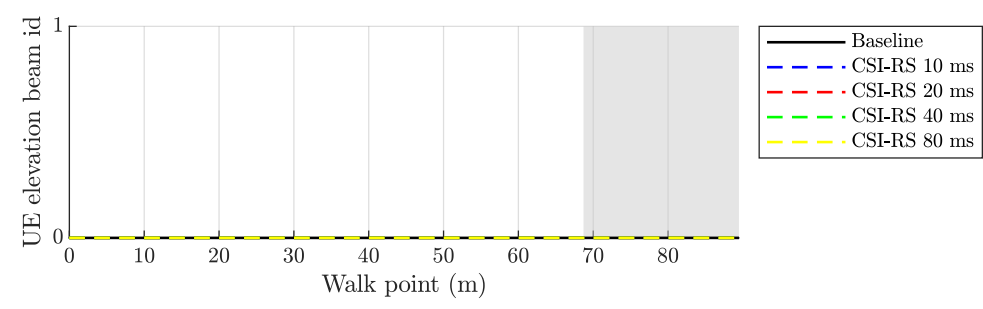

(d) UE elevation beam id.

Figure 5.31: Beam ids changes with long SS burst period and CSI-RS beam tracking in UMi walk at vehicular speed. The greyed area corresponds to NLoS region. 


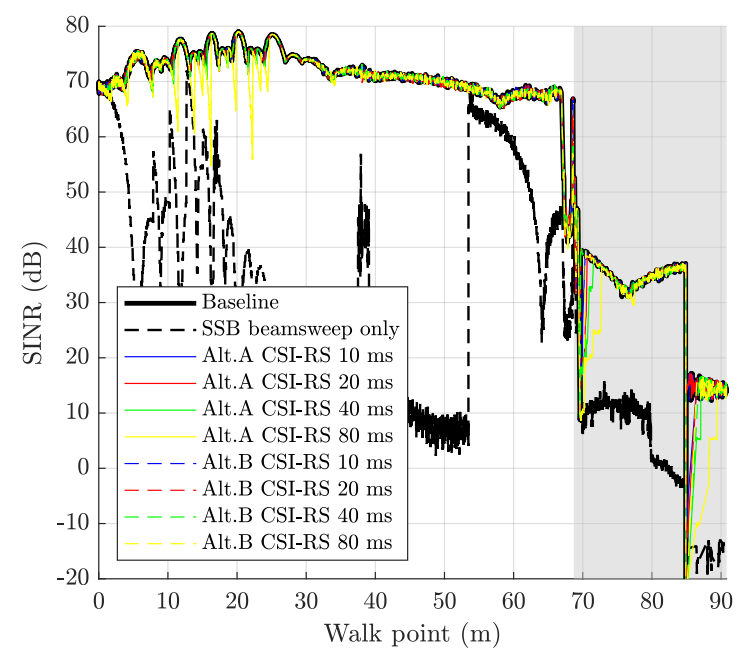

Figure 5.32: SINR evolution in the UMi walk at vehicular speed with long SS burst period.

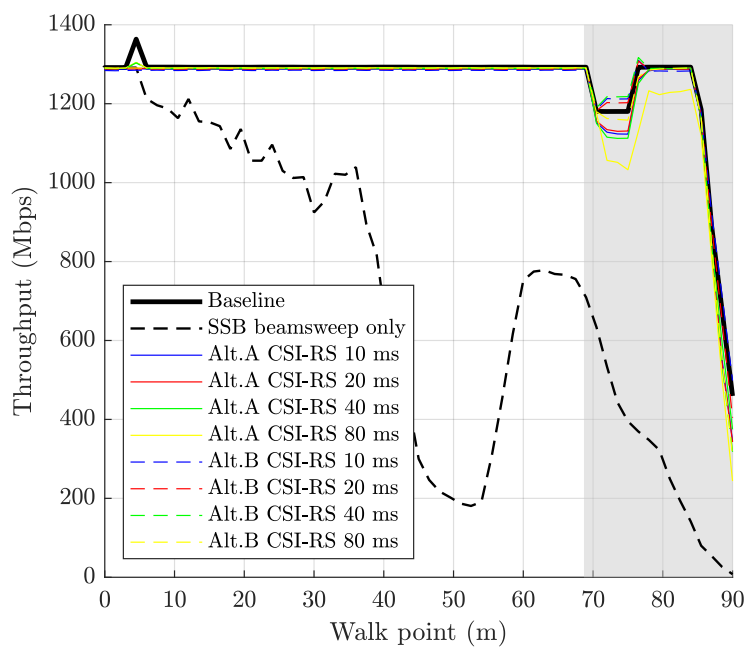

Figure 5.33: User throughput evolution in the UMi walk at vehicular speed with long SS burst period. 
Table 5.15: Average throughput values obtained at vehicular speed for the baseline case and for the beam tracking strategies with different SS burst and CSI-RS periods.

\begin{tabular}{|c|c|c|c|c|c|}
\hline \multirow{2}{*}{ Strategy } & \multirow{2}{*}{$\begin{array}{c}\text { SS burst } \\
\text { period }(\mathrm{ms})\end{array}$} & \multirow{2}{*}{$\begin{array}{c}\text { CSI-RS } \\
\text { period }(\mathrm{ms})\end{array}$} & \multicolumn{3}{|c|}{ Avg. Throughput (Mbps) } \\
\hline & & & All & LoS & NLoS \\
\hline Baseline & - & - & 1254.23 & 1294.09 & 1132.04 \\
\hline \multirow{2}{*}{ SSB-only } & 20 & - & 1169.38 & 1275.83 & 842.94 \\
\hline & 160 & - & 710.01 & 843.55 & 300.50 \\
\hline \multirow{8}{*}{ Alt.A } & \multirow{4}{*}{20} & 10 & 1221.90 & 1260.38 & 1103.88 \\
\hline & & 20 & 1222.36 & 1261.77 & 1101.51 \\
\hline & & 40 & 1221.92 & 1262.36 & 1097.91 \\
\hline & & 80 & 1214.89 & 1263.57 & 1065.64 \\
\hline & \multirow{4}{*}{160} & 10 & 1239.31 & 1278.10 & 1120.33 \\
\hline & & 20 & 1229.75 & 1276.11 & 1087.58 \\
\hline & & 40 & 1237.48 & 1276.12 & 1118.97 \\
\hline & & 80 & 1216.94 & 1277.00 & 1032.78 \\
\hline \multirow{8}{*}{ Alt.B } & \multirow{4}{*}{20} & 10 & 1240.57 & 1288.42 & 1093.83 \\
\hline & & 20 & 1242.08 & 1289.78 & 1095.78 \\
\hline & & 40 & 1242.98 & 1290.69 & 1096.67 \\
\hline & & 80 & 1228.70 & 1291.04 & 1037.54 \\
\hline & \multirow{4}{*}{160} & 10 & 1224.84 & 1257.27 & 1125.41 \\
\hline & & 20 & 1225.60 & 1259.66 & 1121.17 \\
\hline & & 40 & 1222.68 & 1261.83 & 1102.61 \\
\hline & & 80 & 1214.91 & 1262.70 & 1068.35 \\
\hline
\end{tabular}


Table 5.16: Beam alignment ratio and BGL in the UMi scenario at vehicular speed for the baseline case and the beam tracking alternatives with multiple

SS burst and CSI-RS periods.

\begin{tabular}{|c|c|c|c|c|c|c|}
\hline \multirow{2}{*}{ Strategy } & \multirow{2}{*}{$\begin{array}{c}\text { SS burst } \\
\text { period }(\mathrm{ms})\end{array}$} & \multirow{2}{*}{$\begin{array}{c}\text { CSI-RS } \\
\text { period }(\mathrm{ms})\end{array}$} & \multicolumn{3}{|c|}{$\mathbf{R}_{\text {alignment }}$} & \multirow{2}{*}{$\begin{array}{l}\text { BGL } \\
(\mathrm{dB})\end{array}$} \\
\hline & & & Pair & gNB & $\mathbf{U E}$ & \\
\hline Baseline & - & - & 1.00 & 1.00 & 1.00 & 0.00 \\
\hline \multirow{2}{*}{ SSB-only } & 20 & - & 0.41 & 0.50 & 0.70 & 4.03 \\
\hline & 160 & - & 0.14 & 0.26 & 0.32 & 9.53 \\
\hline \multirow{8}{*}{ Alt.A } & \multirow{4}{*}{20} & 10 & 0.85 & 0.86 & 0.99 & 2.93 \\
\hline & & 20 & 0.81 & 0.83 & 0.97 & 2.75 \\
\hline & & 40 & 0.76 & 0.80 & 0.95 & 2.71 \\
\hline & & 80 & 0.71 & 0.75 & 0.92 & 3.26 \\
\hline & \multirow{4}{*}{160} & 10 & 0.85 & 0.86 & 0.99 & 3.01 \\
\hline & & 20 & 0.81 & 0.83 & 0.97 & 2.87 \\
\hline & & 40 & 0.76 & 0.80 & 0.94 & 3.70 \\
\hline & & 80 & 0.67 & 0.75 & 0.88 & 4.36 \\
\hline \multirow{8}{*}{ Alt.B } & \multirow{4}{*}{20} & 10 & 0.86 & 0.86 & 0.99 & 1.69 \\
\hline & & 20 & 0.83 & 0.83 & 0.99 & 1.64 \\
\hline & & 40 & 0.78 & 0.80 & 0.97 & 2.02 \\
\hline & & 80 & 0.72 & 0.75 & 0.94 & 2.71 \\
\hline & \multirow{4}{*}{160} & 10 & 0.86 & 0.86 & 0.99 & 1.79 \\
\hline & & 20 & 0.83 & 0.83 & 0.99 & 1.76 \\
\hline & & 40 & 0.78 & 0.80 & 0.97 & 2.36 \\
\hline & & 80 & 0.72 & 0.75 & 0.94 & 2.78 \\
\hline
\end{tabular}

A CSI-RS period of $80 \mathrm{~ms}$ performs slightly worse than the other options due to the larger beam misalignment probability.

Table 5.16 lists the beam alignment ratio and BGL value for each beam tracking configuration in consideration for the vehicular mobility in UMi. As it can be seen, using the smallest CSI-RS period of $10 \mathrm{~ms}$ is necessary to provide beam alignment ratios close or identical to the values obtained in the ideal baseline case. As the CSI-RS periodicity increases, the beam alignment ratio decreases, with a maximum performance loss of 17\%. Beam tracking Alt.B provides better beam alignment that Alt.A since the extra monitoring beams help to detect the next beam pair faster. In addition, the Alternative B provides smaller BGL. 


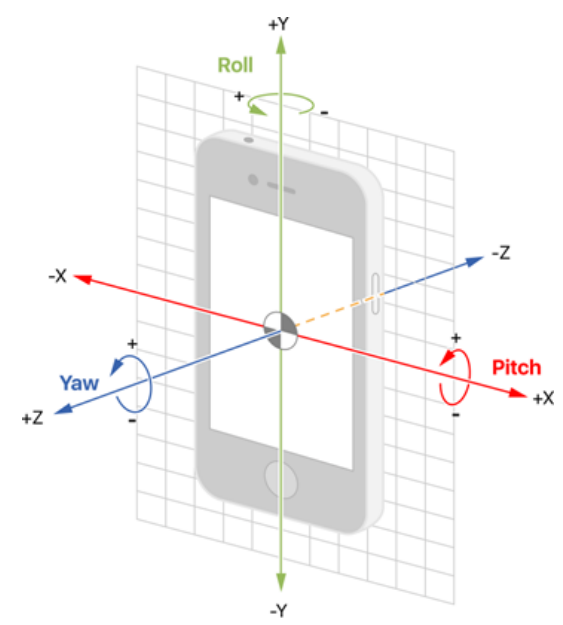

Figure 5.34: Coordinate system of the UE and its rotation axis.

\subsection{Rotation}

This section of the chapter is devoted to the analysis of the effect of the handset orientation in the determination of the optimal pair of beams. The study is centred at UE orientation analysis, since in a cellular deployment the gNB antenna panels are fixed. Instinctively, the optimal pair of beams in a given location must be perceived differently depending on the UE orientation. This study aims at confirming such intuition.

\subsubsection{Coordinate system}

Rotations can be applied to the three axis defined by the plane containing the UE antenna array and the normal direction of the array. The rotation applied can be expressed in Euler angles, which are used to express the rotation applied to a rigid body in a given coordinate system. Taking the notation used in aviation, rotations applied to a mobile handset can be expressed to roll, pitch and yaw as illustrated in Figure 5.34

\subsubsection{Analytical study on beam prints}

As described in Section 5.1.1, the beam print represents the coverage area of the beam id providing the largest beam gain for each point of an area of interest. 


\section{CHAPTER 5. BEAM TRACKING TECHNIQUES}

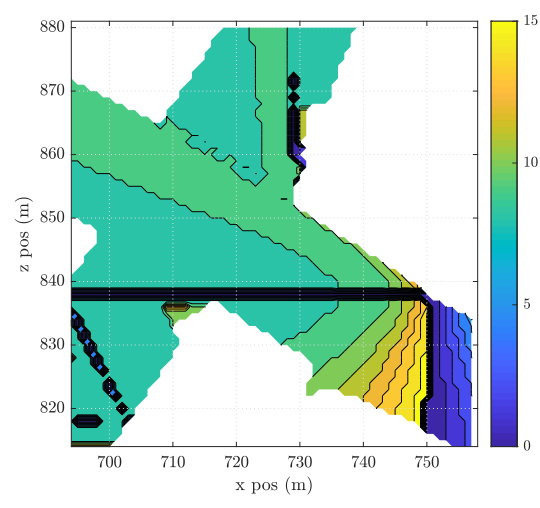

(a) $90^{\circ}$ roll rotation.

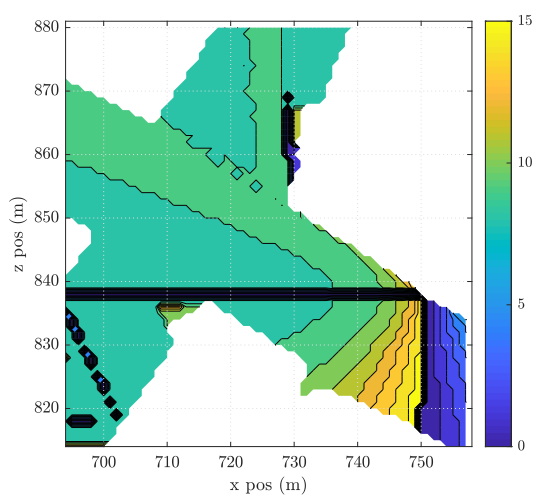

(b) $90^{\circ}$ pitch rotation.

Figure 5.35: gNB azimuth beam prints for roll and pitch rotations.

This is a simple yet effective method to visually identify the best-serving beam in a given area.

For simplicity, the selected scenario to represent beam prints on is the UMi area. For this illustrative exercise, two UE rotations are considered: one rotation of $90^{\circ}$ in roll and another rotation of $90^{\circ}$ in pitch.

Figure 5.35 depicts the gNB azimuth beam prints for the two rotations in consideration; Figure 5.35(a) corresponds to the $90^{\circ}$ rotation in roll and Figure $5.35(\mathrm{~b})$ is for the $90^{\circ}$ rotation in pitch. In the same way, Figure 5.36 illustrates the gNB elevation beam prints; Figure 5.36(a) corresponds to the $90^{\circ}$ rotation in roll and Figure $5.36(\mathrm{~b})$ is for the $90^{\circ}$ rotation in pitch. As it can be seen in both pairs of figures, the differences between gNB beam prints for the two rotations are subtle, and they are only appreciated in the frontier region between two beam prints. In addition, the gNB original beam print in UMi depicted in Figure 5.7 and the beam prints of the two rotation examples are practically identical. This illustrative results confirms the intuition that the orientation of the UE has little influence on the best serving gNB beam.

Continuing with the study of the UE beam prints, Figure 5.37 and Figure 5.38 illustrate the UE beam prints in azimuth and elevation planes, respectively. In the $90^{\circ}$ roll rotation case, the azimuth beam print in Figure 5.37(a) seems rotated with respect to the reference beam print in Figure 5.7(a), and the rotation is similar to the rotation applied to the handset. No changes are appreciated in the elevation beam prints since the pitch has not been changed. However, in case of rotating the handset in pitch, both azimuth and elevation beam prints are altered. The number of UE azimuth beam prints is reduced 


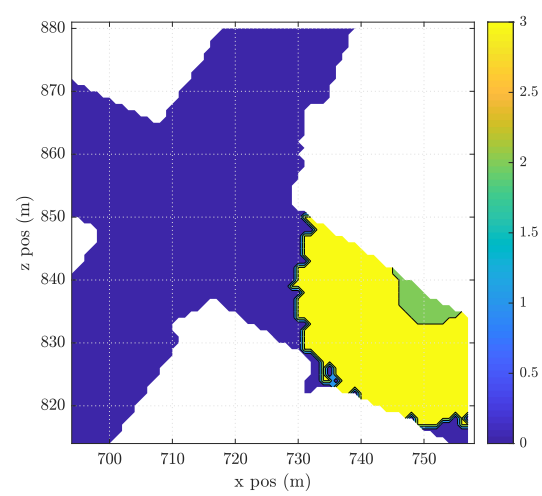

(a) $90^{\circ}$ roll rotation.

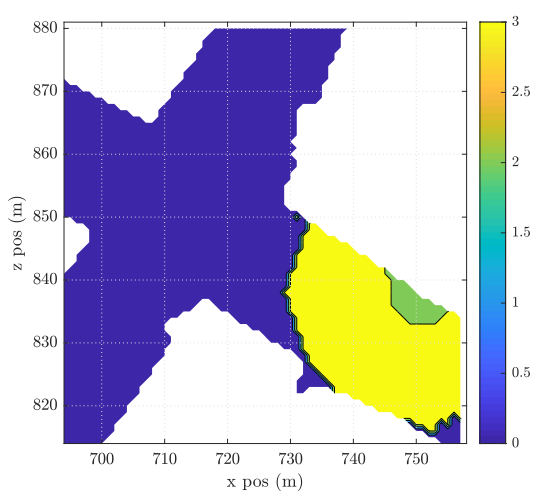

(b) $90^{\circ}$ pitch rotation.

Figure 5.36: gNB elevation beam prints for roll and pitch rotations.

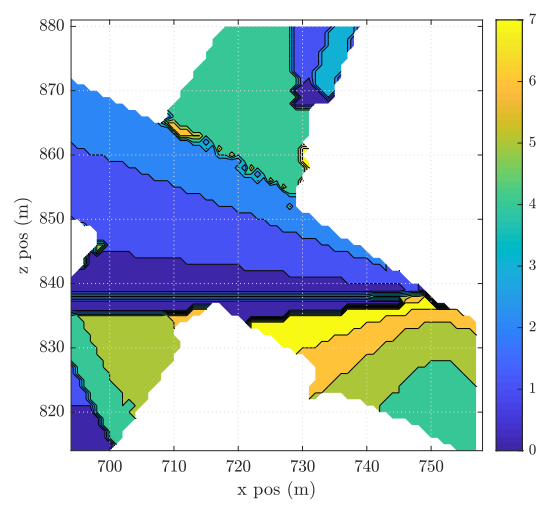

(a) $90^{\circ}$ roll rotation.

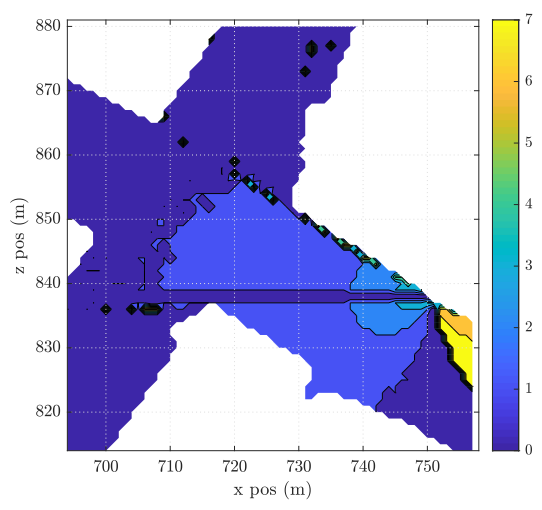

(b) $90^{\circ}$ pitch rotation.

Figure 5.37: UE azimuth beam prints for roll and pitch rotations.

as it can be seen comparing both prints in 5.37(b). The UE elevation beam prints have interchanged areas, and even the elevation beam id is confined in a smaller area in Figure 5.38(b) 


\section{CHAPTER 5. BEAM TRACKING TECHNIQUES}

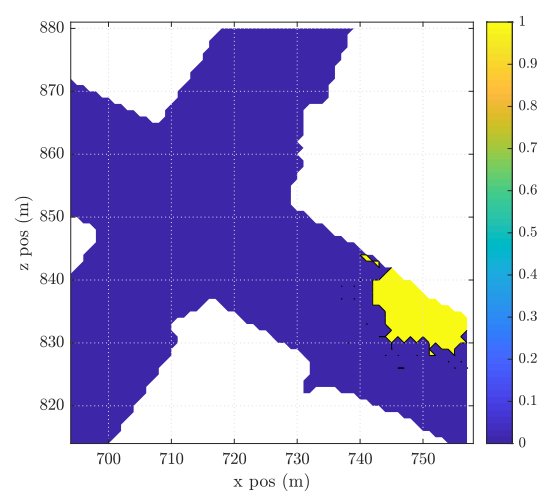

(a) $90^{\circ}$ roll rotation.

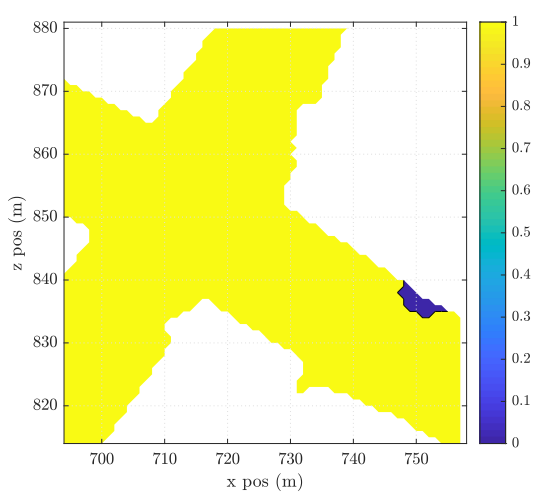

(b) $90^{\circ}$ pitch rotation.

Figure 5.38: UE elevation beam prints for roll and pitch rotations.

\section{Beam distance to reference orientation}

Then, for each location in the scenario, different rotations were applied and then the beam deviation probability and the beam distance with respect to the reference orientation (North) were obtained. Table 5.17 and Table 5.18 summarises the beam deviation metrics of the gNB and UE side respectively for different rotation applied. Note that rotation was applied in rotation steps of $90^{\circ}$ in roll and pitch Euler angles.

The beam deviation probability on the gNB side is relatively small as it can be seen in Table 5.17, with a maximum probability of $5.20 \%$ for rotation of $90^{\circ}$ in both roll and pitch in the positive direction. This results suggest that beam tracking of gNB beams is hardly influenced by the UE, with only a few cases where beams change mainly due to the scenario geometry and the multipath propagation. Beam distances in azimuth is larger than in elevation, due to the fact that more beams are compressed in the same $180^{\circ}$ area in azimuth. Average beam distances range from 2.19 to 4.59 in azimuth, indicating that optimal AoD angles in this plane are ranged within $90^{\circ}$. Beam deviation is rather practically not influenced by the UE rotation, with smaller beam deviations than in azimuth.

Table 5.18 contains the UE beam deviation probability and beam distances for the different rotations applied to the handset. Rotating the device clearly affects the optimal UE beam, but does not affect the azimuth and elevation beams in the same way. The maximum azimuth beam deviation is $85.98 \%$ for most rotations, and the maximum elevation beam deviation is with a $26.16 \%$. 
Table 5.17: Beam deviation probability and beam distance w.r.t. the optimal gNB beams in UMi for different handset rotations (degrees) applied to the reference UE orientation (North).

\begin{tabular}{|r|r|r|r|r|r|}
\hline \multicolumn{2}{|c|}{ Rotation } & \multicolumn{2}{c|}{ gNB azimuth beam } & \multicolumn{2}{c|}{ gNB elevation beam } \\
\hline Roll & Pitch & Probability & Distance & Probability & Distance \\
\hline \hline $90^{\circ}$ & $0^{\circ}$ & $2.31 \%$ & 4.56 & $1.16 \%$ & 1 \\
\hline$-90^{\circ}$ & $0^{\circ}$ & $2.46 \%$ & 4.59 & $1.01 \%$ & 1 \\
\hline $180^{\circ}$ & $0^{\circ}$ & $1.01 \%$ & 3.86 & $0.58 \%$ & 1 \\
\hline $0^{\circ}$ & $90^{\circ}$ & $4.62 \%$ & 2.47 & $2.46 \%$ & 1 \\
\hline $0^{\circ}$ & $-90^{\circ}$ & $4.48 \%$ & 2.65 & $2.17 \%$ & 1 \\
\hline $90^{\circ}$ & $90^{\circ}$ & $5.20 \%$ & 2.19 & $3.61 \%$ & 1 \\
\hline $90^{\circ}$ & $-90^{\circ}$ & $4.19 \%$ & 2.48 & $3.03 \%$ & 1 \\
\hline$-90^{\circ}$ & $90^{\circ}$ & $4.05 \%$ & 2.39 & $2.89 \%$ & 1 \\
\hline$-90^{\circ}$ & $-90^{\circ}$ & $5.06 \%$ & 2.23 & $3.47 \%$ & 1 \\
\hline $180^{\circ}$ & $90^{\circ}$ & $4.34 \%$ & 2.7 & $2.17 \%$ & 1 \\
\hline $180^{\circ}$ & $-90^{\circ}$ & $4.62 \%$ & 2.47 & $2.46 \%$ & 1 \\
\hline
\end{tabular}

Table 5.18: Beam deviation probability and beam distance w.r.t. the optimal UE beams in UMi for different handset rotations (degrees) applied to the reference UE orientation (North).

\begin{tabular}{|r|r|r|r|r|r|}
\hline \multicolumn{2}{|c|}{ Rotation } & \multicolumn{2}{c|}{ UE azimuth beam } & \multicolumn{2}{c|}{ UE elevation beam } \\
\hline Roll & Pitch & Probability & Distance & Probability & Distance \\
\hline \hline $90^{\circ}$ & $0^{\circ}$ & $98.12 \%$ & 2.92 & $8.82 \%$ & 1 \\
\hline$-90^{\circ}$ & $0^{\circ}$ & $92.49 \%$ & 2.56 & $7.51 \%$ & 1 \\
\hline $180^{\circ}$ & $0^{\circ}$ & $85.98 \%$ & 2.37 & $11.85 \%$ & 1 \\
\hline $0^{\circ}$ & $90^{\circ}$ & $47.40 \%$ & 1.35 & $26.16 \%$ & 1 \\
\hline $0^{\circ}$ & $-90^{\circ}$ & $86.13 \%$ & 2.47 & $26.16 \%$ & 1 \\
\hline $90^{\circ}$ & $90^{\circ}$ & $97.11 \%$ & 2.93 & $26.16 \%$ & 1 \\
\hline $90^{\circ}$ & $-90^{\circ}$ & $91.18 \%$ & 2.84 & $26.16 \%$ & 1 \\
\hline$-90^{\circ}$ & $90^{\circ}$ & $91.33 \%$ & 2.84 & $26.16 \%$ & 1 \\
\hline$-90^{\circ}$ & $-90^{\circ}$ & $97.11 \%$ & 2.93 & $26.16 \%$ & 1 \\
\hline $180^{\circ}$ & $90^{\circ}$ & $86.27 \%$ & 2.46 & $26.16 \%$ & 1 \\
\hline $180^{\circ}$ & $-90^{\circ}$ & $47.25 \%$ & 1.36 & $26.16 \%$ & 1 \\
\hline
\end{tabular}




\section{CHAPTER 5. BEAM TRACKING TECHNIQUES}

Beam distances in azimuth shows that beams are between 2 or 3 beams away from the reference orientation. In elevation, beam distances are practically 1.

\subsubsection{UE rotation in walk}

So far, the orientation of the UE in any of the UE walks was set to the North and fixed along the whole walk. This modelling assumption involves that the UE maintains its original orientation even after turning the street. This time, the walk exercise is repeated but the orientation of the device will change after it turns the corner. The rotation applied to the UE orientation corresponds to the turning angle. Note that the rotation applied is in azimuth since rotation is applied over the normal direction to the ground.

\section{UMa walk at pedestrian speed}

The objective of this exercise is illustrating how the beam id changes after a rotation is applied. To that aim, and for the sake of simplicity, only the pedestrian mobility is considered.

Assuming an SS burst period of $160 \mathrm{~ms}$ and a CSI-RS period of $10 \mathrm{~ms}$, Figure 5.39 represents the beam pair evolution of the original UMi walk for the reference (solid lines) and rotated (dashed lines) orientations. The figure illustrates the beam pair changes along the walk for the baseline case, and Alternative A and Alternative B beam tracking strategies.

The user turns to its right at point 53.4 metres from the starting point. As a consequence of orientation change, the azimuth UE beam drifts 5 beam ids with respect to the reference orientation. The gNB beams, however, do not experience any change after rotating the device. There is, indeed, a beam change in the azimuth gNB beams at point 47 metres, prior to the device rotation. Therefore, this beam change is due to intrinsic channel characteristics. Moreover, the beam switch is fast enough to be only captured by the baseline case, which channel update period is $1 \mathrm{~ms}$.

Table 5.19 aims at comparing the performance of the beam tracking ability for for the reference orientation $(\mathbf{N})$ and with rotation $(\mathbf{R})$ in the UMa walk. As it can be seen, the beam alignment ratio and the BGL obtained in the two orientations are practically identical. These results emphasise the adaptability of the beam tracking Alternative A and Alternative B to overcome beam shifts due to a change in the orientation of the user device. 


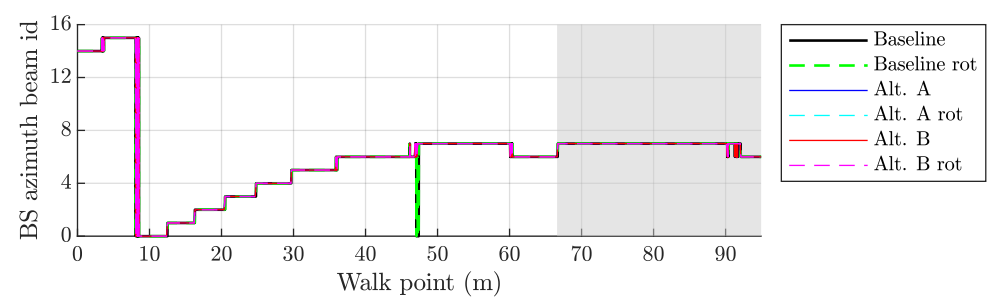

(a) gNB azimuth beam id.

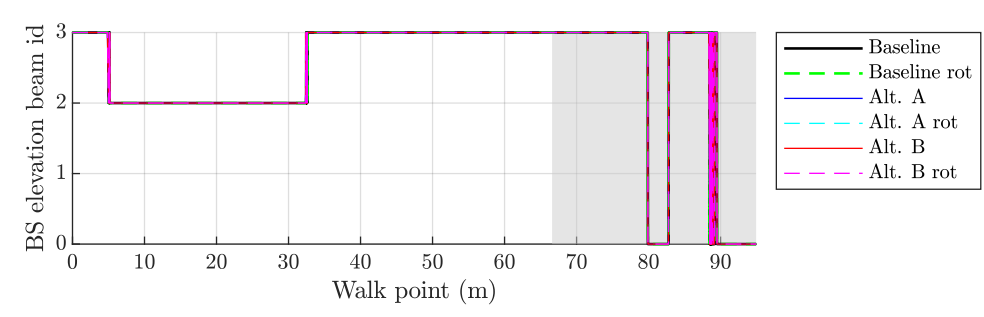

(b) gNB elevation beam id.

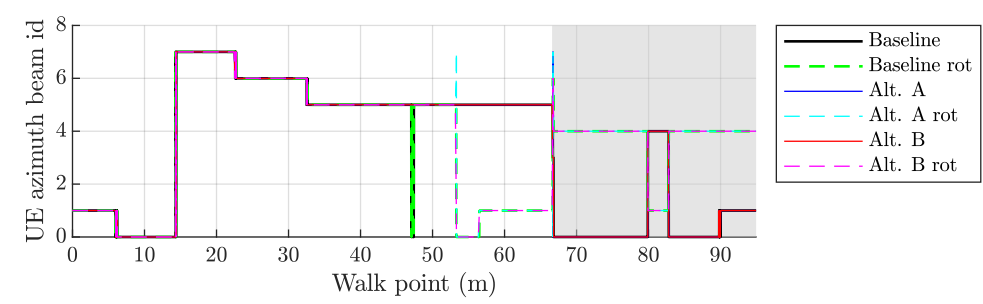

(c) UE azimuth beam id.

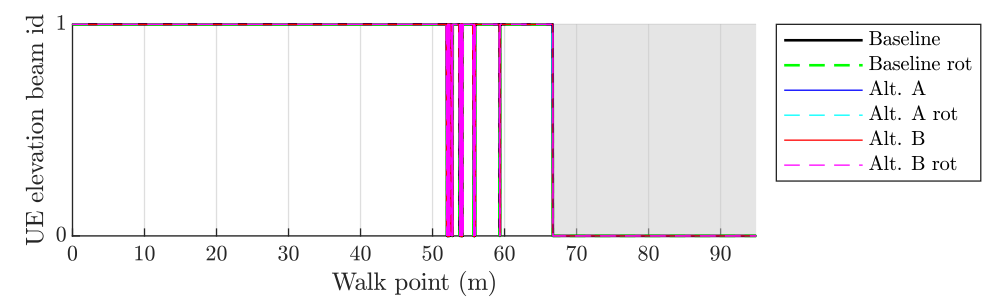

(d) UE elevation beam id.

Figure 5.39: Beam id changes in UMa walk at pedestrian speed for a baseline and beam tracking alternatives A and B with the SS burst period set to 160 $\mathrm{ms}$ and the CSI-RS period set to $10 \mathrm{~ms}$. 
Table 5.19: Beam alignment ratio and BGL in the UMa scenario for the reference orientation (N) and with rotation $(\mathbf{R})$ at pedestrian speed for the beam tracking alternatives with multiple SS burst and CSI-RS periods.

\begin{tabular}{|c|c|c|c|c|c|c|c|c|c|c|}
\hline \multirow{3}{*}{ Strategy } & \multirow{3}{*}{$\begin{array}{c}\text { SS burst } \\
\text { period } \\
(\mathrm{ms})\end{array}$} & \multirow{3}{*}{$\begin{array}{c}\text { CSI-RS } \\
\text { period } \\
(\mathrm{ms})\end{array}$} & \multicolumn{6}{|c|}{$\mathbf{R}_{\text {alignment }}$} & \multirow{2}{*}{\multicolumn{2}{|c|}{$\begin{array}{l}\text { BGL } \\
\text { (dB) }\end{array}$}} \\
\hline & & & \multicolumn{2}{|c|}{ Pair } & \multicolumn{2}{|c|}{ gNB } & \multicolumn{2}{|c|}{$\overline{\mathbf{U E}}$} & & \\
\hline & & & $\mathbf{N}$ & $\mathbf{R}$ & $\mathbf{N}$ & $\mathbf{R}$ & $\mathbf{N}$ & $\mathbf{R}$ & $\mathbf{N}$ & $\mathbf{R}$ \\
\hline \multirow{2}{*}{ SSB-only } & 20 & - & 0.90 & 0.91 & 0.96 & 0.97 & 0.93 & 0.93 & 2.58 & 2.69 \\
\hline & 160 & - & 0.62 & 0.67 & 0.81 & 0.82 & 0.74 & 0.77 & 4.78 & 6.46 \\
\hline \multirow{8}{*}{ Alt.A } & \multirow{4}{*}{20} & 10 & 0.98 & 0.98 & 0.99 & 0.99 & 0.99 & 0.99 & 2.19 & 2.54 \\
\hline & & 20 & 0.97 & 0.98 & 0.99 & 0.99 & 0.98 & 0.99 & 2.47 & 2.89 \\
\hline & & 40 & 0.97 & 0.97 & 0.99 & 0.99 & 0.98 & 0.98 & 2.41 & 2.92 \\
\hline & & 80 & 0.96 & 0.96 & 0.98 & 0.98 & 0.97 & 0.97 & 2.45 & 3.38 \\
\hline & \multirow{4}{*}{160} & 10 & 0.98 & 0.98 & 0.99 & 0.99 & 0.99 & 0.99 & 2.19 & 2.50 \\
\hline & & 20 & 0.97 & 0.98 & 0.99 & 0.99 & 0.98 & 0.99 & 2.45 & 2.84 \\
\hline & & 40 & 0.97 & 0.97 & 0.99 & 0.99 & 0.98 & 0.98 & 3.37 & 4.20 \\
\hline & & 80 & 0.96 & 0.96 & 0.98 & 0.98 & 0.97 & 0.98 & 4.69 & 5.51 \\
\hline \multirow{8}{*}{ Alt.B } & \multirow{4}{*}{20} & 10 & 0.98 & 0.98 & 0.99 & 0.99 & 0.99 & 0.99 & 1.36 & 1.77 \\
\hline & & 20 & 0.98 & 0.98 & 0.99 & 0.99 & 0.99 & 0.99 & 1.53 & 1.97 \\
\hline & & 40 & 0.97 & 0.97 & 0.99 & 0.99 & 0.98 & 0.98 & 1.79 & 2.42 \\
\hline & & 80 & 0.96 & 0.96 & 0.98 & 0.98 & 0.97 & 0.97 & 2.04 & 2.86 \\
\hline & \multirow{4}{*}{160} & 10 & 0.98 & 0.98 & 0.99 & 0.99 & 0.99 & 0.99 & 1.35 & 1.74 \\
\hline & & 20 & 0.98 & 0.98 & 0.99 & 0.99 & $\overline{0.99}$ & 0.99 & 1.52 & 1.92 \\
\hline & & 40 & 0.97 & 0.97 & 0.99 & 0.99 & 0.98 & 0.98 & 2.03 & 2.66 \\
\hline & & 80 & 0.97 & 0.97 & 0.98 & 0.98 & 0.98 & 0.98 & 3.10 & 3.81 \\
\hline
\end{tabular}




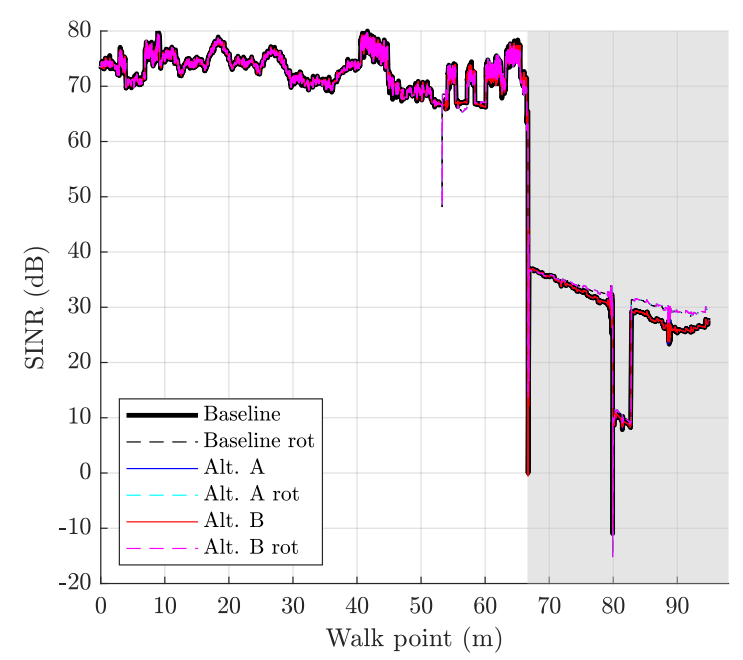

Figure 5.40: SINR evolution of the former and rotated walks in UMa for the CSI-RS beam tracking alternatives.

Despite of the different UE beam id trends after rotation, the perceived SINR is not penalised as illustrated in Figure 5.40. Initially, rotation reduces the experienced SINR due to the beam misalignment. However, the duration in time of this misalignment situation is very limited, and the SINR is quickly recovered with any of the two beam tracking alternatives. After rotation, not only does rotation not reduce the SINR, but it can increase the quality of the channel as occurs after point 75 metres. The motivation is the increased beam gain in the rotated case. More interestingly, the transition to NLoS with rotation is not as critical as with the former orientation and the reduction of SINR is $18 \mathrm{~dB}$ smaller.

Figure 5.41 illustrates the user throughput in the UMa walk at pedestrian speed for the same beam tracking use cases. As it can be seen, the SINR reduction at point 53.4 metres after rotation does not penalise the throughput since the SINR is still high to maintain the same modulation and bit rate, and this holds for the transition from LoS to NLoS. The different SINR levels in the different use cases and the congestion window management influences on the throughput recovery after walk point 80 metres. 


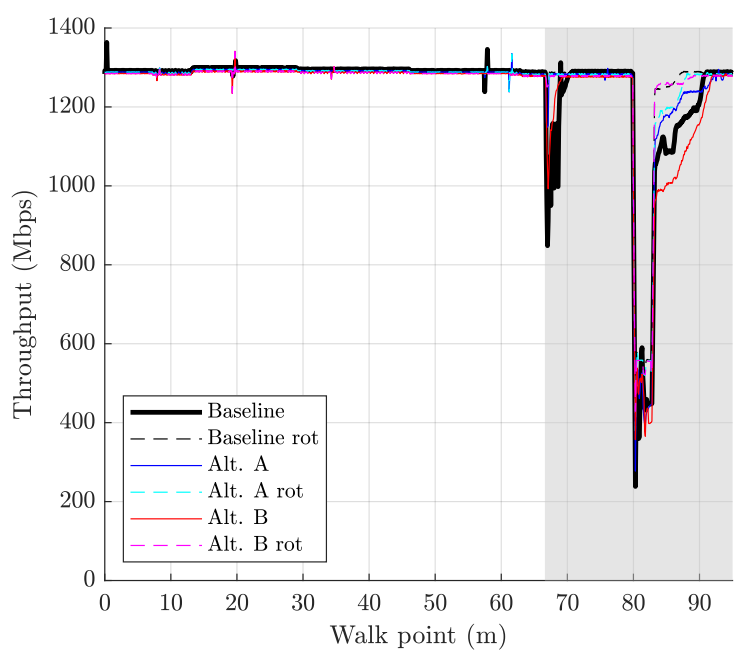

Figure 5.41: Throughput evolution of the former and rotated walks in UMa for the CSI-RS beam tracking alternatives.

\section{UMi walk at pedestrian speed}

Continuing the rotation analysis with the UMi scenario, Figure 5.42 illustrates the beam pair evolution of the original and rotated UMi walks, represented with solid and dashed lines respectively. As in the UMa case, the beam tracking configuration was set to an SS burst period of $160 \mathrm{~ms}$ and a CSI-RS period of $10 \mathrm{~ms}$. As it can be seen in Figure 5.42(a) and Figure 5.42(b), the gNB trend is not influenced by the change in the orientation, and it is difficult to determine by visual inspection the point where the orientation changes. There are two exceptions in the walk points 68 metres and 85 metres in Figure 5.42(a), which are detected with the baseline configuration only. Therefore, these beam changes are residual and can only be detected with the very small time resolution.

The turning point occurs at walk point 56.3 metres and the UE rotates in azimuth. This change in the orientation of the UE becomes clear in the azimuth beam plot in Figure 5.42(c). After rotating the terminal, the azimuth UE beam id curves separate from those obtained with the reference orientation. No differences are appreciated in UE elevation beams in the whole walk. 


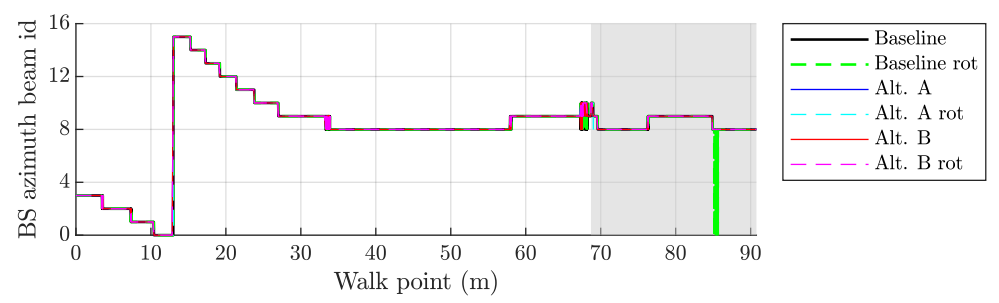

(a) gNB azimuth beam id.

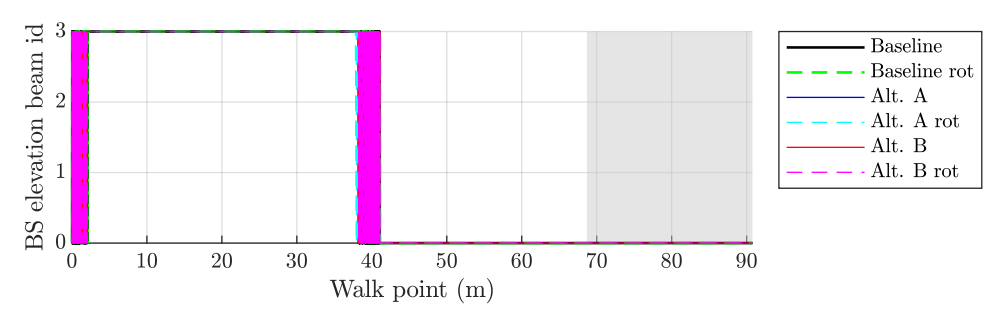

(b) gNB elevation beam id.

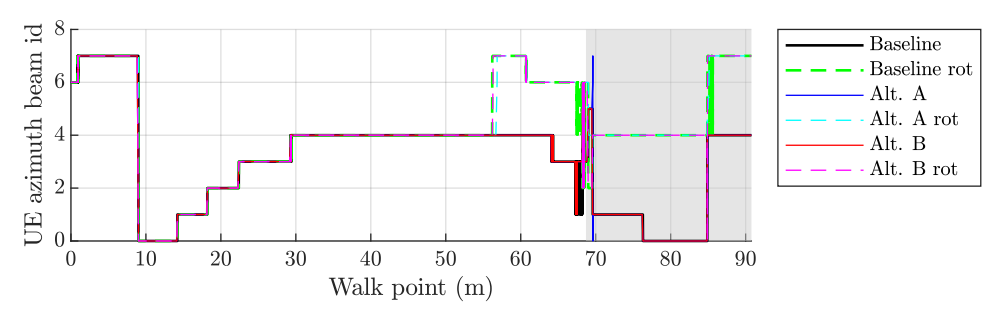

(c) UE azimuth beam id.

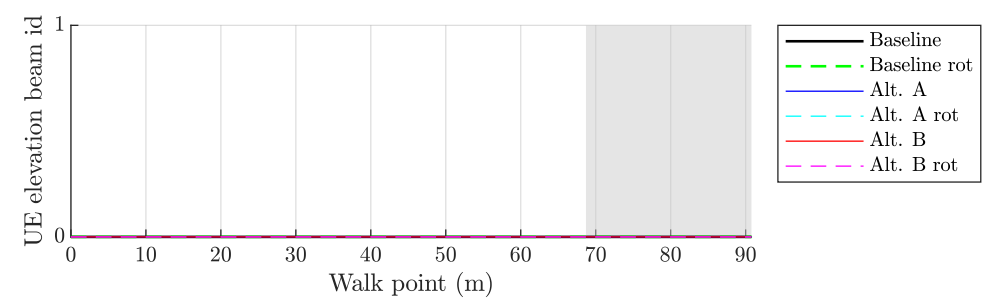

(d) UE elevation beam id.

Figure 5.42: Beam id changes in UMi walk at pedestrian speed for a baseline and beam tracking alternatives A and B with the SS burst period set to $160 \mathrm{~ms}$ and the CSI-RS period set to $10 \mathrm{~ms}$. 
Table 5.20: Beam alignment ratio and BGL in the UMi scenario for the reference orientation (N) and with rotation $(\mathbf{R})$ at pedestrian speed for the beam tracking alternatives with multiple SS burst and CSI-RS periods.

\begin{tabular}{|c|c|c|c|c|c|c|c|c|c|c|}
\hline \multirow{3}{*}{ Strategy } & \multirow{3}{*}{$\begin{array}{c}\text { SS burst } \\
\text { period } \\
(\mathrm{ms})\end{array}$} & \multirow{3}{*}{$\begin{array}{c}\text { CSI-RS } \\
\text { period } \\
(\mathrm{ms})\end{array}$} & \multicolumn{6}{|c|}{$\mathbf{R}_{\text {alignment }}$} & \multirow{2}{*}{\multicolumn{2}{|c|}{$\begin{array}{l}\text { BGL } \\
\text { (dB) }\end{array}$}} \\
\hline & & & \multicolumn{2}{|c|}{ Pair } & \multicolumn{2}{|c|}{ gNB } & \multicolumn{2}{|c|}{$\overline{\mathbf{U E}}$} & & \\
\hline & & & $\mathbf{N}$ & $\mathbf{R}$ & $\mathbf{N}$ & $\mathbf{R}$ & $\mathbf{N}$ & $\mathbf{R}$ & $\mathbf{N}$ & $\mathbf{R}$ \\
\hline \multirow{2}{*}{ SSB-only } & 20 & - & 0.90 & 0.91 & 0.96 & 0.97 & 0.93 & 0.93 & 2.58 & 2.69 \\
\hline & 160 & - & 0.62 & 0.67 & 0.81 & 0.82 & 0.74 & 0.77 & 4.78 & 6.46 \\
\hline \multirow{8}{*}{ Alt.A } & \multirow{4}{*}{20} & 10 & 0.98 & 0.98 & 0.99 & 0.99 & 0.99 & 0.99 & 2.19 & 2.54 \\
\hline & & 20 & 0.97 & 0.98 & 0.99 & 0.99 & 0.98 & 0.99 & 2.47 & 2.89 \\
\hline & & 40 & 0.97 & 0.97 & 0.99 & 0.99 & 0.98 & 0.98 & 2.41 & 2.92 \\
\hline & & 80 & 0.96 & 0.96 & 0.98 & 0.98 & 0.97 & 0.97 & 2.45 & 3.38 \\
\hline & \multirow{4}{*}{160} & 10 & 0.98 & 0.98 & 0.99 & 0.99 & 0.99 & 0.99 & 2.19 & 2.50 \\
\hline & & 20 & 0.97 & 0.98 & 0.99 & 0.99 & 0.98 & 0.99 & 2.45 & 2.84 \\
\hline & & 40 & 0.97 & 0.97 & 0.99 & 0.99 & 0.98 & 0.98 & 3.37 & 4.20 \\
\hline & & 80 & 0.96 & 0.96 & 0.98 & 0.98 & 0.97 & 0.98 & 4.69 & 5.51 \\
\hline \multirow{8}{*}{ Alt.B } & \multirow{4}{*}{20} & 10 & 0.98 & 0.98 & 0.99 & 0.99 & 0.99 & 0.99 & 1.36 & 1.77 \\
\hline & & 20 & 0.98 & 0.98 & 0.99 & 0.99 & 0.99 & 0.99 & 1.53 & 1.97 \\
\hline & & 40 & 0.97 & 0.97 & 0.99 & 0.99 & 0.98 & 0.98 & 1.79 & 2.42 \\
\hline & & 80 & 0.96 & 0.96 & 0.98 & 0.98 & 0.97 & 0.97 & 2.04 & 2.86 \\
\hline & \multirow{4}{*}{160} & 10 & 0.98 & 0.98 & 0.99 & 0.99 & 0.99 & 0.99 & 1.35 & 1.74 \\
\hline & & 20 & 0.98 & 0.98 & 0.99 & 0.99 & $\overline{0.99}$ & 0.99 & 1.52 & 1.92 \\
\hline & & 40 & 0.97 & 0.97 & 0.99 & 0.99 & 0.98 & 0.98 & 2.03 & 2.66 \\
\hline & & 80 & 0.97 & 0.97 & 0.98 & 0.98 & 0.98 & 0.98 & 3.10 & 3.81 \\
\hline
\end{tabular}




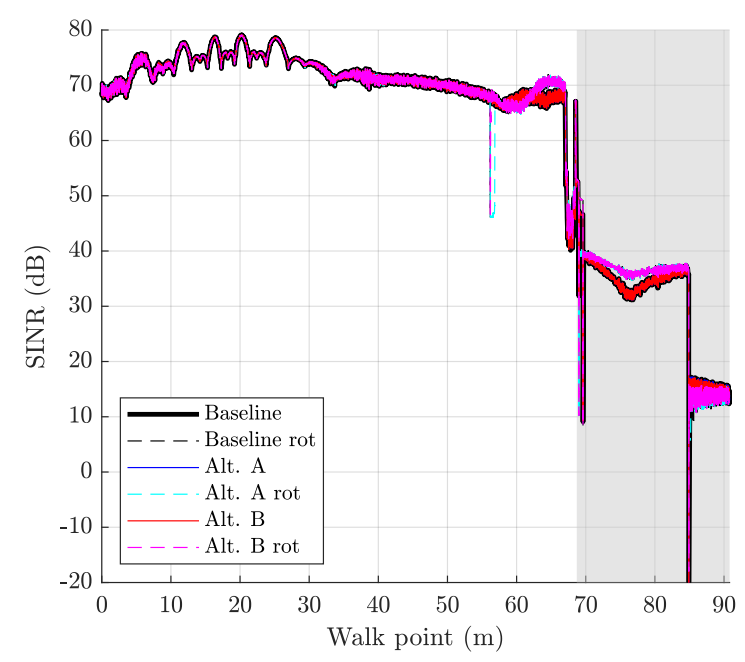

Figure 5.43: SINR evolution of the former and rotated walks in UMi for the CSI-RS beam tracking alternatives.

The difference in UE beams after rotating the device has an implication in the perceived SINR levels due to the change of beam gains, as illustrated in Figure 5.43. But in general, beam tracking Alternative A and Alternative B provide good excellent SINR levels, independently of the device orientation, emphasising the adaptability of the beam tracking solution in an event of change in the orientation. This adaptability has a positive influence on the throughput experienced by the user. Figure 5.44 shows stable user data rate in LoS and NLoS, with the exception of the transition from LoS to NLoS, and after point 85 metres where the SINR is drastically reduced to an average value of $17 \mathrm{~dB}$ for the reference orientation and $15 \mathrm{~dB}$ for the new orientation. These levels are insufficient for the peak user throughput.

\subsection{Conclusions}

In this chapter of the Thesis, the beam tracking strategies based on measuring Reference Signal (RS) have been presented. Two urban scenarios and tracking evaluation metrics have been proposed. In general terms, they have proven to work reasonably well in the evaluation scenarios presented in this chapter. 


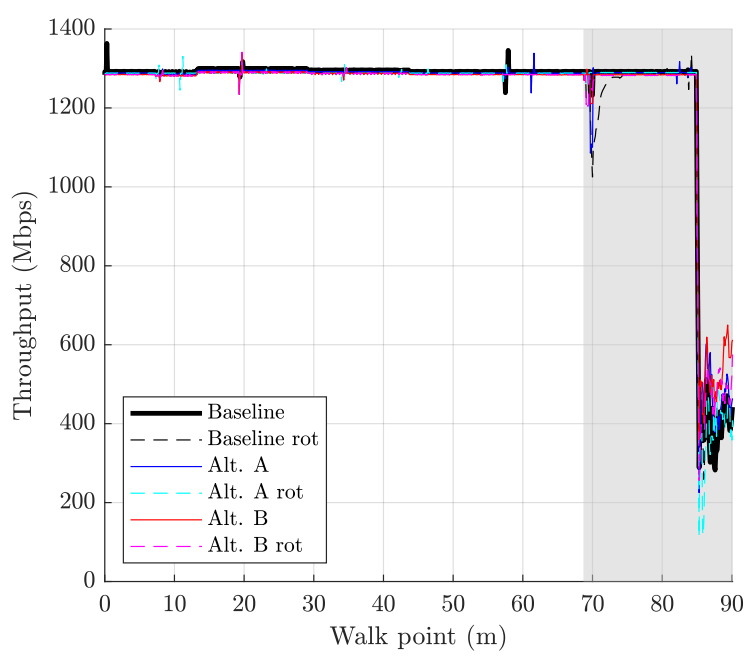

Figure 5.44: Throughput evolution of the former and rotated walks in UMi for the CSI-RS beam tracking alternatives.

Tracking based on beam sweeping works well in situations not requiring frequent beam updates. This is the case of LoS situations. It has been shown that perfect beam tracking is achieved in UE walks at low speed. However, SSB-based beam tracking fails in scenarios with abrupt beam changes due to the large delay detecting the new optimal pair of beams with beam sweeping. The beam sweeping speed is determined by the total number of beams, the SS burst period and the number of SSB transmission occasions within the SS burst.

Beam tracking with SSBs has been proved to work in a specific situation which is not unique in an urban environment. To enable faster beam updates to better capture beam transitions when necessary, the NR standard contemplates the use of CSI-RS resources. But the NR standard does not mandate any strategy on how to manage the beam information nor to perform the beam tracking. This chapter of the thesis has introduced two beam tracking strategies based on CSI-RS beam measurements. Alternative A monitors beams which are contiguous to the active beams in the spatial domain, and Alternative $\mathrm{B}$ extends the prior alternative by tracking additional equally-spaced azimuth beams. The envisaged strategies have been evaluated in different urban scenarios under different beam tracking configurations and mobility profiles. Different combinations of SS burst and CSI-RS periods have been tested. 
At pedestrian speed, the beam tracking Alternative A and Alternative B provide similar throughput and beam alignment ratio for equal SS burst period. At this speed, and with a LoS condition, the SS burst period could be enlarged without reducing the beam alignment in order to maximise the throughput by reducing the signalling overhead due to SSB. This cannot be done in NLoS situations, where the average user throughput worsens with SS burst period of $40 \mathrm{~ms}$ in UMa and $80 \mathrm{~ms}$ in UMi.

In the pedestrian mobility case, the beam tracking strategy Alternative A and Alternative B perform similarly well in terms of throughput and beam alignment ratio, making it difficult to decide which alternative is best in the particular urban scenarios evaluated. This statement holds for any CSI-RS periodicity.

On the other hand, the simulation results at vehicular speed show that Alternative B provides extra beam tracking resilience in NLoS situations compared to Alternative A. And the better alignment provides better throughput in NLoS. This time, the selection of the CSI-RS period influences the beam tracking ability, and the shortest period has reported the best beam tracking performance and throughput. Therefore, Alternative B with $10 \mathrm{~ms}$ CSI-RS period could be used as a one-fits-all beam tracking solution for a $5 \mathrm{G}$ network operating in the mmWave band. A future evolution of the beam management could dynamically switch strategies between one or another based on the visibility condition or other kind of considerations. But this sort of information sometimes cannot be extracted from beam measurements.

With respect to the UE orientation analysis, beam deviation metrics with respect to the orientation of reference have been defined and their values have been averaged out through the area of interest. Results show that, in general, the optimal beams on the gNB side are practically unaffected by the device rotation, with a maximum deviation probability of $5.20 \%$, and differences are concentrated in very located small areas. However, the UE orientation drastically changes the optimal beam on the UE side. Roll rotations are very likely to change the optimal azimuth UE beams. This is reasonable since the antenna is oriented in a different azimuth direction. Pitch rotations have been shown to be more critical than azimuth rotations. Pitch rotations can completely change both azimuth and elevation UE beams as shown in the fingerprints. The beam tracking strategies based on measuring RS presented in this chapter of the Thesis have proved to track modifications in the device orientation due to their adaptability, striving to maximise the perceived SINR and the user throughput. Certainly, this ability makes the beam tracking alternatives indicated for situations with high rotation probabilities. 



\section{Chapter 6}

\section{Contextual Beam Tracking Techniques}

The performance of Millimeter Wave (mmWave) systems can be severely hampered by a poor selection of transmit and receive beams. An effective beam tracking technique must provide means to always know which are the best beams. In Chapter 5, the technique studied consisted in the continuous tracking of a list of candidate beams using standard Synchronization Signal (SS)/Physical Broadcast Channel (PBCH)-Block (SSB) and Channel State Information Reference Signals (CSI-RS) in the vicinity of the active pair of beams. The solutions detailed in Section 5.3.1 have shown to be effective in a specific interference-free scenario where a single user is getting all resources from the network. However, in a real deployment multiple users may be served simultaneously by a single mmWave Base Station (BS). All those users should be allocated user-specific CSI-RS to obtain and report channel knowledge. As a consequence, and with the beam tracking strategies in Chapter 5, the signalling overhead due to CSI-RS would exhaust the resources available in the BS if the number of served users increased.

In this scenario, the introduction of contextual information about the scenario in the beam tracking decision-making process may help reduce the list of beams to monitor and, consequently, save resources. For example, the user location can be of great help. At a given point in a dynamic scenario, the multipath channel in different time realisations shall be very similar among them, with some frequently-repeated directions or equivalent beams. The orientation of the user terminal, or the user speed, are also some examples of contextual information which can be useful for beam tracking. 
This chapter of the Thesis is intended as an initial study of beam tracking strategies which make use of contextual information. The study focuses on two different approaches:

- Section 6.1 introduces a new beam tracking method based on a inverse fingerprinting database, which makes suggestions on the beam pairs to to monitor based on the orientation and location reported by the User Equipment (UE). Firstly, the section details the aspects of a possible implementation of this beam tracking strategy. Secondly, the system model assumptions are given, with special focus on modelling imperfections of the orientation and location determination. Then simulation results are given for the inverse fingerprinting approach with and without errors in the determination of the contextual information. Finally, the main conclusions of this beam tracking strategy are drawn.

- Section 6.2 presents a predictive model for beam tracking built upon a machine learning model. This section dives into the formal aspects of a Neural Network (NN) and proposes a Multi-Layer Perceptron (MLP) model to estimate the next pair of beams to use based on the last optimal beam pairs reported by the user. This section finishes with the main conclusions on the machine learning model for beam tracking.

\subsection{Inverse fingerprinting for beam tracking}

The first attempt of giving some sort of contextual information to the network about the scenario and the radio environment in this Thesis is by means of managing an up-to-date database which, for each UE orientation and location in the scenario, indicates the set of beam pairs to monitor.

\subsubsection{Mechanism description}

The beam tracking implementation is different to the one based on CSI-RS and described in Section 5.3. The fundamental difference with the former strategy is that beam pair suggestions are obtained from consulting the inverse fingerprinting database rather than configuring beam reports on neighbouring beams.

The beam tracking strategy with inverse fingerprinting works as follows. Once the UE is in CONNECTED state and a first set of useful beams has been detected using SSB beam sweeping, the serving New Radio (NR) base station (gNB) instructs the UE to provide their location coordinates with the upper layer Location Request message. Details on the Location Request message 
are given in Section 2.2.3. The UE location information is made available at the gNB so it configures the next set of CSI-RS resources to monitor which correspond to the reported UE location. Note that the number of CSI-RS resources and, hence, the number of beam pairs to monitor is configurable. Figure 6.1 illustrates the possible message exchange flow of one iteration of the inverse fingerprinting beam tracking strategy with the UE reporting its location to the gNB.

The UE location information can be estimated at the gNB. For instance, the user location can be inferred from the historical of measurement reports collected at the gNB. This may eliminate the requirement of having the UE explicitly reporting its location. In addition, the user location estimation by the gNB may be necessary for the inverse fingerprinting beam tracking in case the user blocks location reporting in the configuration parameters in its device.

The UE measures the beamformed CSI-RS resources and reports the beam quality metrics as configured. The network adjusts the serving beams if necessary and restarts the inverse fingerprinting beam tracking by requesting a new UE location determination in order to detect a necessary change of the list of beam pairs to monitor. For simplicity and control signalling reduction, the periodic location request can be configured, and the periodicity should preferably match the CSI-RS reporting period. This procedure is repeated as long as the UE remains in the CONNECTED state.

\subsubsection{System model assumptions}

The system model for inverse fingerprinting beam tracking assumes that the database has been populated beforehand with all the information required to perform the beam tracking at any point in the area of interest of an urban scenario. Since this is a complicated task, which requires a huge collection of measurements in a wide range of situations, this Thesis assumes that a fingerprinting database is obtained from the analytical evaluation of the channel response as described in Section 3.1. And the channel responses were obtained from the ray tracing tool described in Section 3.2.2. The spatial resolution of the database is the same used in the generation of the channel responses.

The inverse fingerprinting beam tracking takes advantage of CSI-RS framework to monitor a list of beam pairs. For each location, the database contains a list with the most promising beam pairs based on the beam gain obtained from the analytical inspection of the channel response. When constructing the list, the maximum number of beam pairs to monitor is 20, although less resources can be allocated if the number of promising beam pairs is lower than this threshold. 

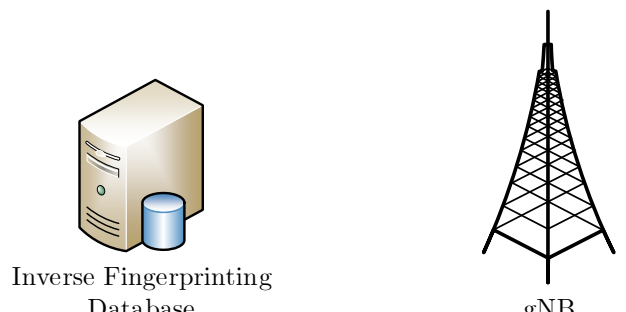

Database

gNB

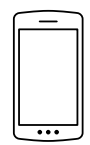

UE

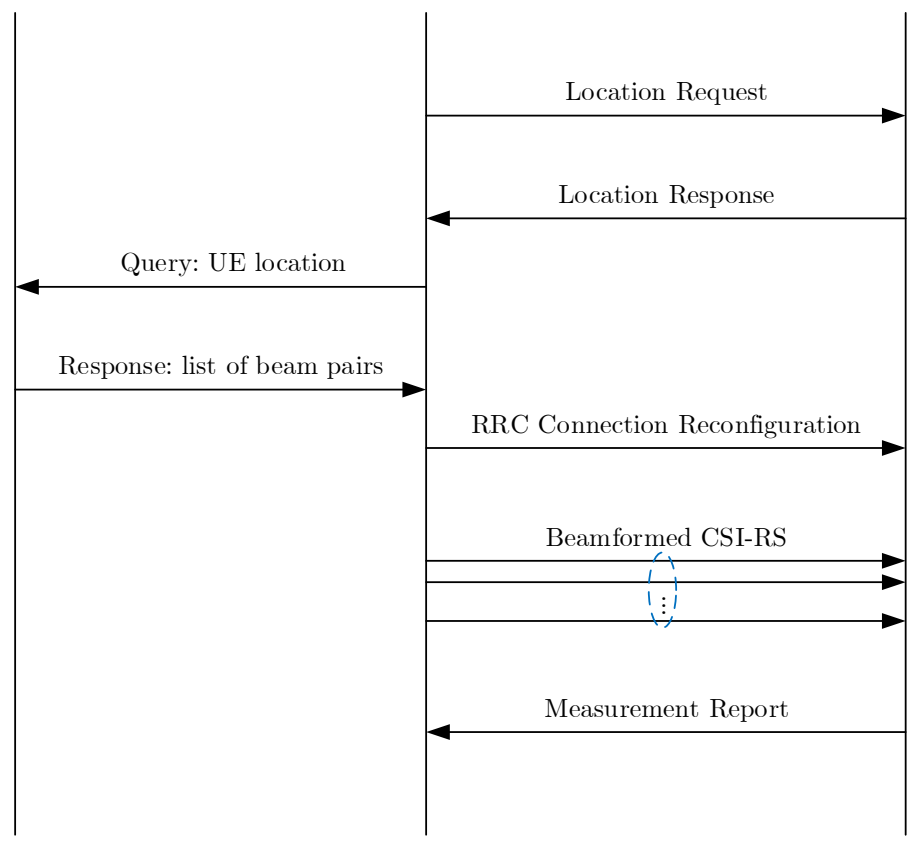

Figure 6.1: Messages exchanged in the inverse fingerprinting beam tracking among the serving gNB, the UE and the local database at the gNB. 
For simplicity, it is assumed that the UE obtains its location information immediately. As a result, the time required to obtain and report the location information is negligible. No resources are consumed in this ideal location information report.

The user device orientation and location might be erroneous. In the following subsections the effect of the orientation and location errors are introduced.

\section{Study on the orientation estimation error}

The orientation of a rigid body can be determined with the output of some specific electronic components. Basically, the output signal of this kind of components depends on the natural forces influencing on them. For example, gyroscopes, or gyros, measure the angular speed of the rotation applied. Accelerometers, on the other hand, measure the projection of the gravity force direction over their axis. The iPhone 4 was the first smartphone in integrating this kind of components, and the vast majority of modern cellular phones sold worldwide are equipped with electronic components helping determine the orientation of the device.

Accelerometers and gyroscopes suffer from zero-force bias, which is the output signal of the device when no force is applied. Price and precision determine the selection of the chip to implement in the system [110]. Smartphones typically implement low-cost chips, which are subject of inaccuracies and errors. Despite of this, low-cost chips provide the necessary accuracy levels for typical mobile applications.

Chip manufacturers do not provide a value in degrees of the orientation error. This information must be inferred from the zero-force bias and other relevant specifications provided in the product data-sheet. As an example, and without loss of generality, Analog Devices chip manufacturer reports a maximum deviation of $3^{\circ}$ for one of their accelerometers [111].

Assuming $3^{\circ}$ as a reference maximum error, and for the sake of assessing the impact of the error in the best beam determination, a sweep on the orientation error is done from $-3^{\circ}$ to $3^{\circ}$ with steps of $0.5^{\circ}$ in yaw and pitch axis as done in Section 5.4. Figure 6.2 represents the probability of the orientation error modifying the optimal gNB beam for the area of interest in Urban Macro-cell (UMa) (blue curve) and Urban Micro-cell (UMi) (red curve) scenarios. For each scenario, curves with circle marker correspond to rotation errors in yaw and curves with squared marker correspond to errors in pitch orientation. As it can be seen, the error probability decreases as orientation deviation approximates to 0 . Still, the largest error probability is as little as $1.44 \%$. This is consistent with the results shown in Section 5.4, where the gNB beam prints were practically unaffected by the orientation of the UE. 


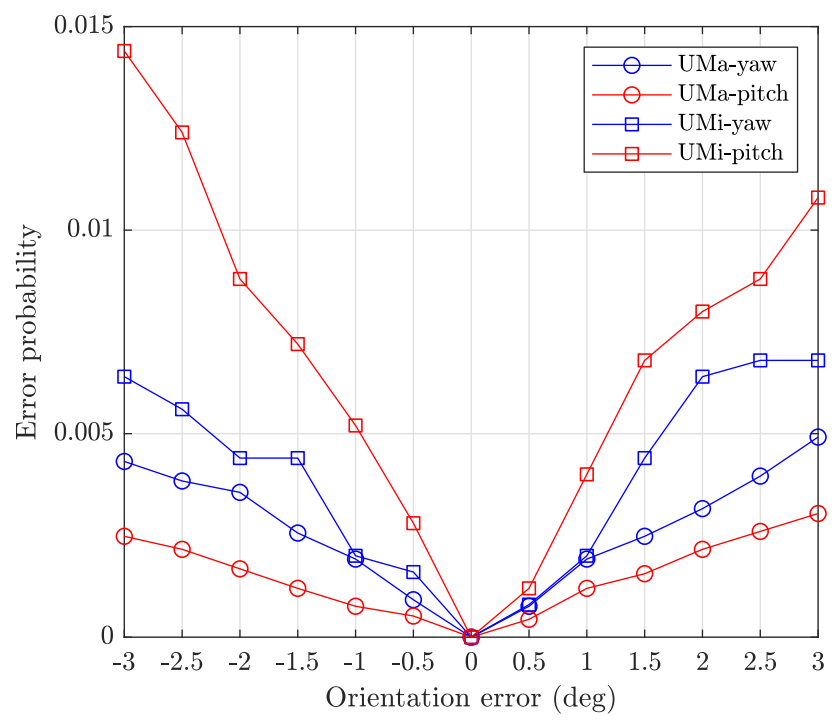

Figure 6.2: Probability of changing the optimal gNB beam due to orientation errors for the two urban scenarios in consideration.

Figure 6.3 represents the probability of the orientation error modifying the optimal UE beam for the scenarios and orientation error values in yaw and pitch orientation. In this case, the probability is 10 times larger than the orientation error probability on the gNB beam, with a maximum error probability of $17 \%$, and errors in yaw orientation are more likely to change the UE beam.

Figure 6.4 and Figure 6.5 illustrate the average beam distance between the beams obtained with orientation errors and the ideal situation with no errors. The definition of the beam distance metric is available in Section 5.1.2. This time, the average beam distances represented in the figures consider those points where the orientation error changes the optimal beam id. Moreover, beam distances are given in absolute values and they are not separated into azimuth and elevation planes.

In both gNB and UE, angular deviations in yaw provide smaller beam distance than pitch deviations. This is due to the fact that the elevation beam is maintained with the rotation in azimuth and consecutive beams ids correspond to consecutive beams in the same azimuth plane. Angular deviations in yaw may change the elevation beam id, and beams ids in elevation are not consecutive and are separated in proportion to the number of azimuth beams. In terms of elevation beams, the maximum gNB elevation beam distance is 2 . 


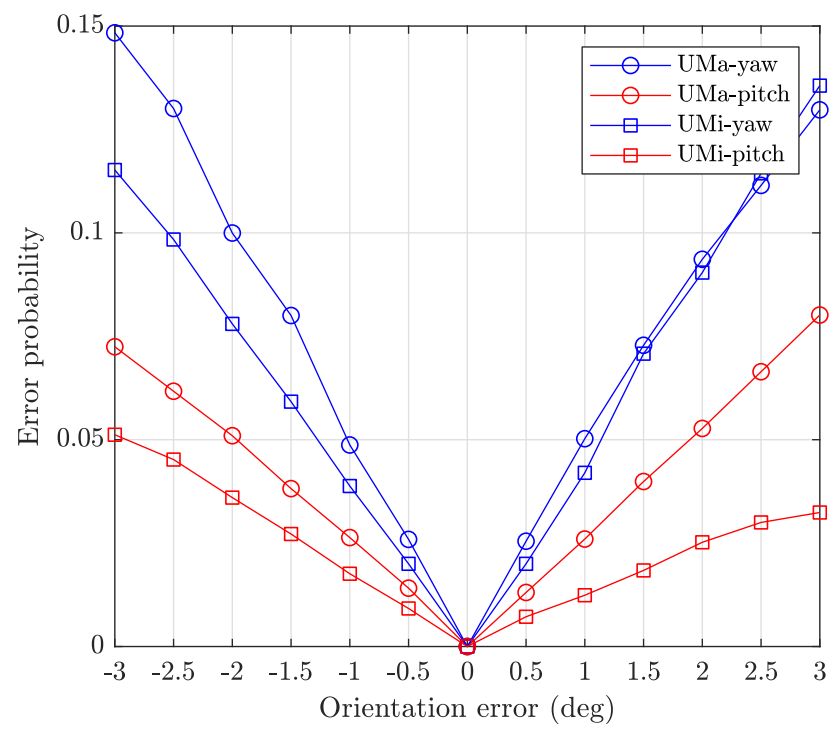

Figure 6.3: Probability of changing the optimal UE beam due to orientation errors for the two urban scenarios in consideration.

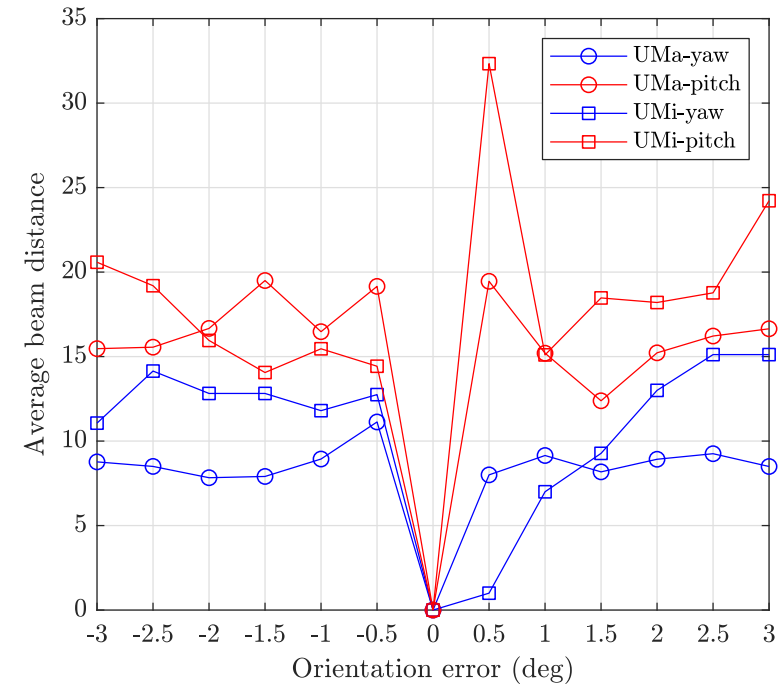

Figure 6.4: Average gNB beam distance between the beam ids with orientation errors and the optimal beam (no error). 


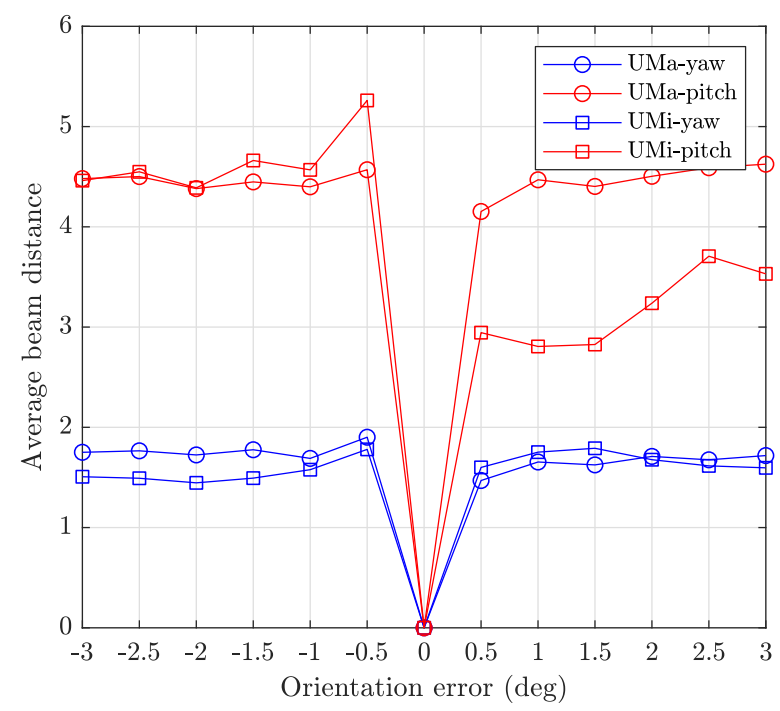

Figure 6.5: Average UE beam distance between the beam ids with orientation errors and the optimal beam (no error).

Despite of the large beam distances of the gNB beams, the error probability is low as illustrated in Figure 6.2.

\section{Study on the location error}

It is well known that Global Navigation Satellite System (GNSS) systems such as Global Positioning System (GPS) suffer from imprecise location estimations in urban canyon scenarios, which are the dominant situations in urban scenarios like in lower Manhattan [112]. The main problem is the severe signal blocking phenomena due to tall buildings and the scarcity of useful signals arriving from satellites, forming a poor location resolution.

Authors in [113] survey the location error obtained with GPS in real urban scenarios. They report an average cellular network user location error around $11.5 \mathrm{~m}$ in a $90 \%$ confidence area with GPS signal only. This result is near the minimum accuracy of the GPS technology for civilian use, which is $7.8 \mathrm{~m}$ in the $95 \%$ confidence interval at ground level as reported by the American government [114]. Authors in [113] propose using an extended Kalman filter to improve the location accuracy, obtaining an error of 3 metres using GPS data.

Authors in [113] also provide typical location errors using cellular network signals in urban scenarios, and reported values between 73 metres and 125 me- 
tres, and the use of their extended Kalman filter method to increase the accuracy provides estimation errors of 73 metres with cellular signals, which can be lowered to 65 metres combined with GPS.

\subsubsection{Simulation results with error-free measurements}

In this part of the assessment, the beam tracking with inverse fingerprinting is evaluated assuming that location and orientation information is accurate and free of errors. The study has focused on the UMi scenario described in 5.1.1 for practical motivations, since CSI-RS beam tracking has proved to perform similarly in UMa and UMi scenarios. Several simulations have been run in ns-3 according to the simulation parameters in Table 5.1.1 for UMi scenario. The obtained beam tracking performance is compared with the baseline case described in Section 5.1.3, and the CSI-RS beam tracking Alternative A and Alternative B defined in Section 5.3.1.

\section{Urban micro scenario at pedestrian speed}

Figure 6.6(a) and Figure 6.6(b) illustrate the gNB and UE beam id evolution for the longest SS burst period in the UMi scenario at pedestrian speed, respectively. From a visual inspection of both figures, it is clear that the beam tracking ability of the inverse fingerprinting beam tracking is similar to the baseline case, with few deviations.

The beam alignment performance of the inverse fingerprinting beam tracking is evaluated in terms of beam alignment ratio and Beam Gain Loss (BGL), which definitions can be found in Section 5.1.2. Table 6.1 contains the performance metrics obtained with a SS burst period of $20 \mathrm{~ms}$, and Table 6.2 contains the same performance metrics for the longest SS burst value of $160 \mathrm{~ms}$. Both beam alignment ratio and BGL metrics are obtained taking the baseline case as reference. Note that the two tables list results for the SSB beam sweeping procedure, the beam tracking Alternative A and B described in Section 5.3.1, and the inverse fingerprinting beam tracking approach. As it can be appreciated in both tables, the beam alignment ratio of the fingerprinting strategy is practically identical to the performance of Alternative $\mathrm{B}$, and slightly better than Alternative A, for both SS burst periods, with a beam alignment probability larger than $95 \%$. The reasons of having practically $100 \%$ probability of beam alignment with the optimal beams is the combination of having an inverse fingerprinting database with accurate beam information for each location along the UE path and the low user speed. Note that the inverse fingerprinting database contains up to 20 pair of beams, including the optimal pair, which can be frequently monitored with CSI-RS measurements. This provides beam 

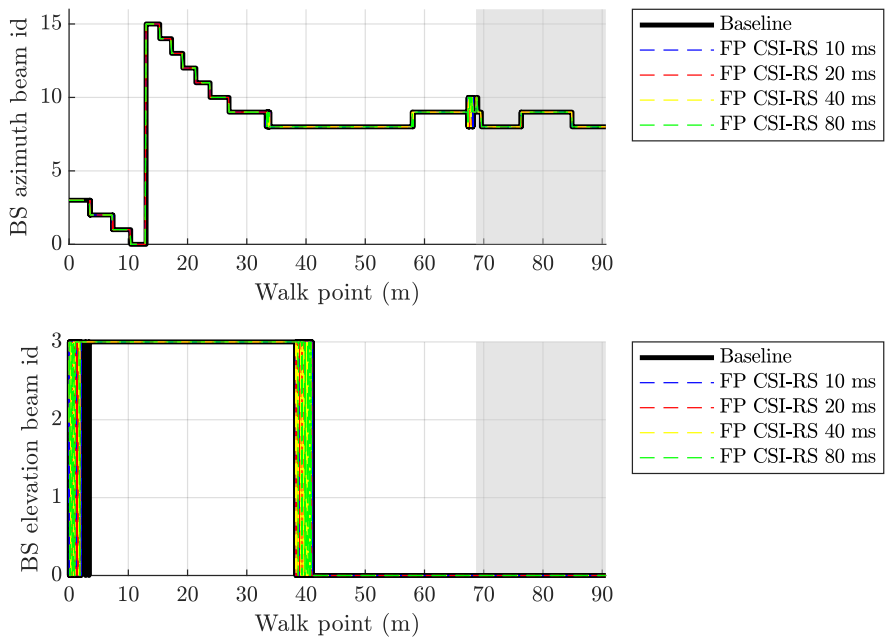

(a) gNB azimuth and elevation beams. .
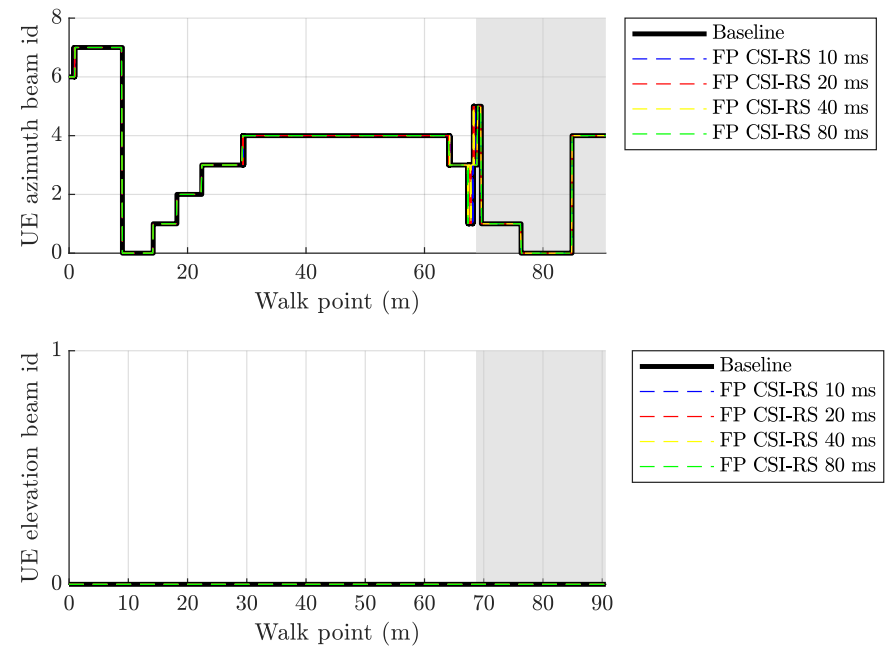

(b) UE azimuth and elevation beams.

Figure 6.6: Beam ids changes for the longest SS burst period and different CSI-RS periods at pedestrian speed. 
Table 6.1: Beam alignment ratio and BGL assuming an SS burst period of 20 ms and pedestrian mobility.

\begin{tabular}{|c|c|c|c|c|c|}
\hline \multirow{2}{*}{ Strategy } & \multirow{2}{*}{$\begin{array}{c}\text { CSI-RS } \\
\text { period }(\mathrm{ms})\end{array}$} & \multicolumn{3}{|c|}{$\mathbf{R}_{\text {alignment }}$} & \multirow{2}{*}{$\begin{array}{l}\text { BGL } \\
(\mathrm{dB})\end{array}$} \\
\hline & & Pair & gNB & UE & \\
\hline SSB-only & - & 0.89 & 0.92 & 0.96 & 2.00 \\
\hline \multirow{4}{*}{ Fingerprinting } & 10 & 0.96 & 0.96 & 0.99 & 1.58 \\
\hline & 20 & 0.95 & 0.96 & 0.99 & 1.65 \\
\hline & 40 & 0.95 & 0.95 & 0.99 & 1.78 \\
\hline & 80 & 0.95 & 0.95 & 0.99 & 1.85 \\
\hline \multirow{4}{*}{ Alt.A } & 10 & 0.96 & 0.96 & 0.99 & 1.76 \\
\hline & 20 & 0.95 & 0.96 & 0.99 & 1.96 \\
\hline & 40 & 0.95 & 0.96 & 0.99 & 2.37 \\
\hline & 80 & 0.94 & 0.95 & 0.98 & 2.58 \\
\hline \multirow{4}{*}{ Alt.B } & 10 & 0.96 & 0.96 & 1.00 & 1.58 \\
\hline & 20 & 0.95 & 0.96 & 0.99 & 1.63 \\
\hline & 40 & 0.95 & 0.95 & 0.99 & 1.77 \\
\hline & 80 & 0.95 & 0.95 & 0.99 & 1.86 \\
\hline
\end{tabular}

redundancy in situations where the beam quality of the most promising pair of beams decreases.

The inverse fingerprinting beam tracking strategy achieves low BGL values due to the excellent beam tracking ability. In general, fingerprinting and beam tracking Alternative B can achieve practically identical performance levels. Alternative A provides slightly worse performance, with a maximum difference of $1.09 \mathrm{~dB}$ obtained with the longest SS burst and CSI-RS periods. The advantage of the fingerprinting strategy over Alternative A and Alternative B is that the good beam tracking performance is achieved with the same amount of CSI-RS resources used Alternative A.

Figure 6.7 illustrates the Signal to Interference plus Noise Ratio (SINR) and throughput evolution of the inverse fingerprinting beam tracking with a fixed SS burst period of $160 \mathrm{~ms}$ and different CSI-RS periodicities. According to Figure 6.7(a), the SINR curves are practically identical to the baseline. As a result, the experienced throughput is also identical to the baseline in Line of Sight (LoS) and very similar in Non Line of Sight (NLoS). The transition to NLoS has an impact on the instant user throughput which is very limited in time. Once recovered, the peak throughput is maintained in the NLoS area where SINR is larger than $17 \mathrm{~dB}$. After this, throughput differences among CSI-RS periodicities are due to the different SINR levels, which has an impact 
Table 6.2: Beam alignment ratio and BGL assuming an SS burst period of $160 \mathrm{~ms}$ and pedestrian mobility.

\begin{tabular}{|c|r|r|r|r|r|}
\hline \multirow{2}{*}{ Strategy } & \multicolumn{1}{|c|}{$\begin{array}{c}\text { CSI-RS } \\
\text { period (ms) }\end{array}$} & \multicolumn{3}{|c|}{$\mathbf{R}_{\text {alignment }}$} & BGL \\
\cline { 2 - 5 } & & Pair & gNB & UE & $(\mathbf{d B})$ \\
\hline \hline SSB-only & - & 0.62 & 0.70 & 0.81 & 4.34 \\
\hline \hline \multirow{4}{*}{ Fingerprinting } & 10 & 0.96 & 0.96 & 0.99 & 1.52 \\
\cline { 2 - 5 } & 20 & 0.95 & 0.96 & 0.99 & 1.62 \\
\cline { 2 - 5 } & 40 & 0.95 & 0.95 & 0.99 & 1.81 \\
\hline \hline \multirow{4}{*}{ Alt.A } & 80 & 0.95 & 0.95 & 0.99 & 1.89 \\
\cline { 2 - 5 } & 10 & 0.96 & 0.96 & 0.99 & 1.70 \\
\cline { 2 - 5 } & 20 & 0.95 & 0.96 & 0.99 & 1.96 \\
\hline \hline \multirow{4}{*}{ Alt.B } & 40 & 0.95 & 0.96 & 0.99 & 2.42 \\
\cline { 2 - 5 } & 80 & 0.94 & 0.95 & 0.99 & 2.98 \\
\cline { 2 - 5 } & 10 & 0.96 & 0.96 & 1.00 & 1.51 \\
\cline { 2 - 5 } & 20 & 0.95 & 0.96 & 0.99 & 1.61 \\
\cline { 2 - 5 } & 40 & 0.95 & 0.95 & 0.99 & 1.79 \\
\hline
\end{tabular}

on the Congestion Window (CW) and Round Trip Time (RTT) as it can be seen in Figure 6.8.

Figure 6.8(a) illustrates the evolution in time of the CW and Figure 6.8(b) shows the RTT in 70 metres around the LoS to NLoS transition. Due to the SINR degradation, the CW shuts and reduces its capacity. Since less packets are transmitted, the RTT increases. But the SINR is rapidly recovered, the CW slowly re-opens, and RTT slowly recovers. At walk point 85 metres, SINR is substantially reduced and the CW progressively closes at different rates depending on the CSI-RS. From this point, the baseline case closes faster than the inverse fingerprinting beam tracking configurations, which results in a smaller baseline throughput rate during the last 5 metres of the simulation.

Table 6.3 and Table 6.4 provide the average throughput at pedestrian speed for the SS burst periods of $20 \mathrm{~ms}$ and $160 \mathrm{~ms}$, respectively. Results are given for the whole UE walk, LoS and NLoS. Both tables contain the average values of the baseline case, SSB beam sweeping and the beam tracking strategies described in Chapter 5 .

In comparison with beam tracking Alternative $\mathrm{A}$ and Alternative $\mathrm{B}$, the inverse fingerprinting beam tracking provides similar performance in terms of average throughput in both tables. Note that signalling overhead is practically determined by number of SSB transmissions in a scenario with a single user and 


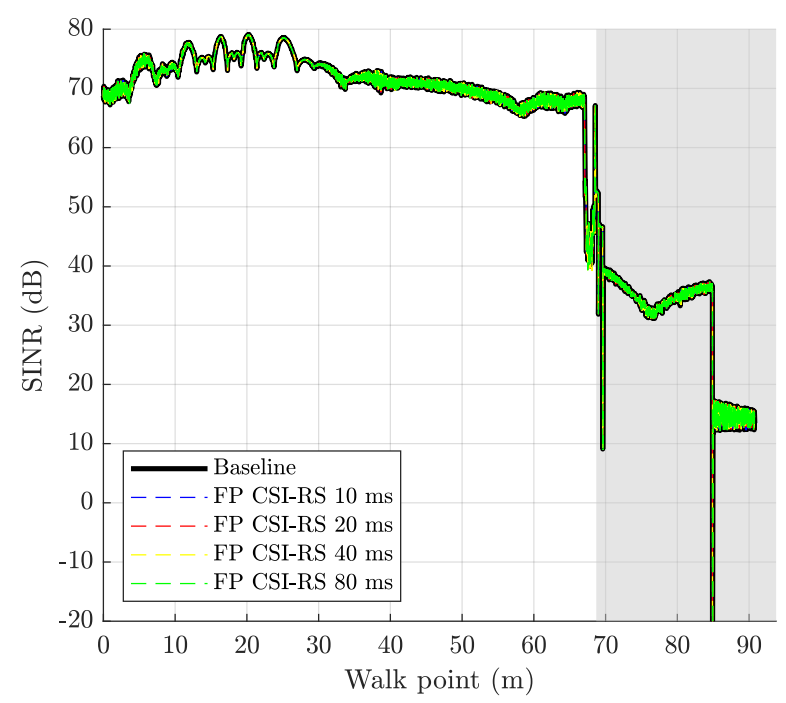

(a) SINR.

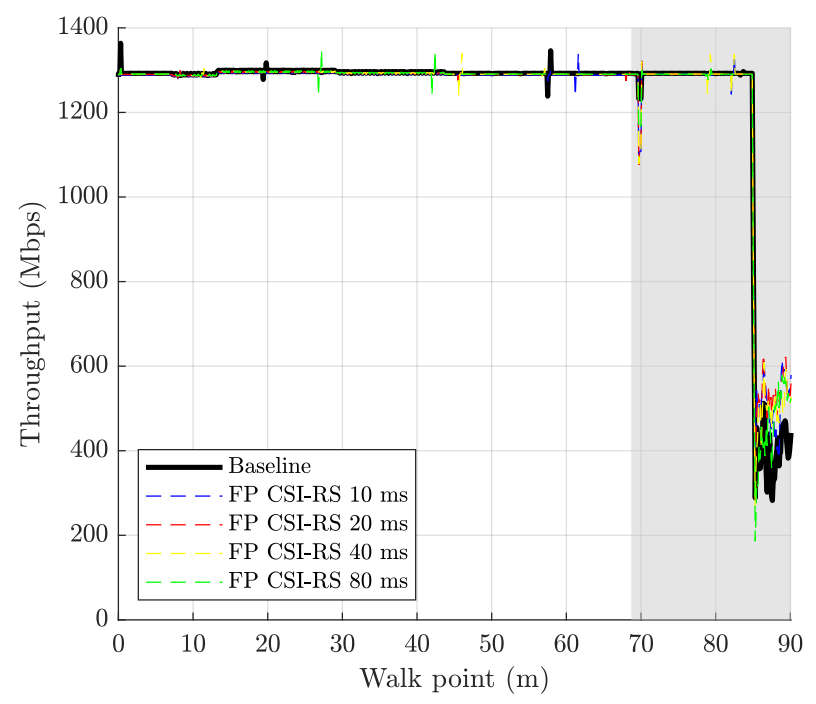

(b) Throughput.

Figure 6.7: Experienced SINR and user throughput with inverse fingerprinting beam tracking at pedestrian speed. 


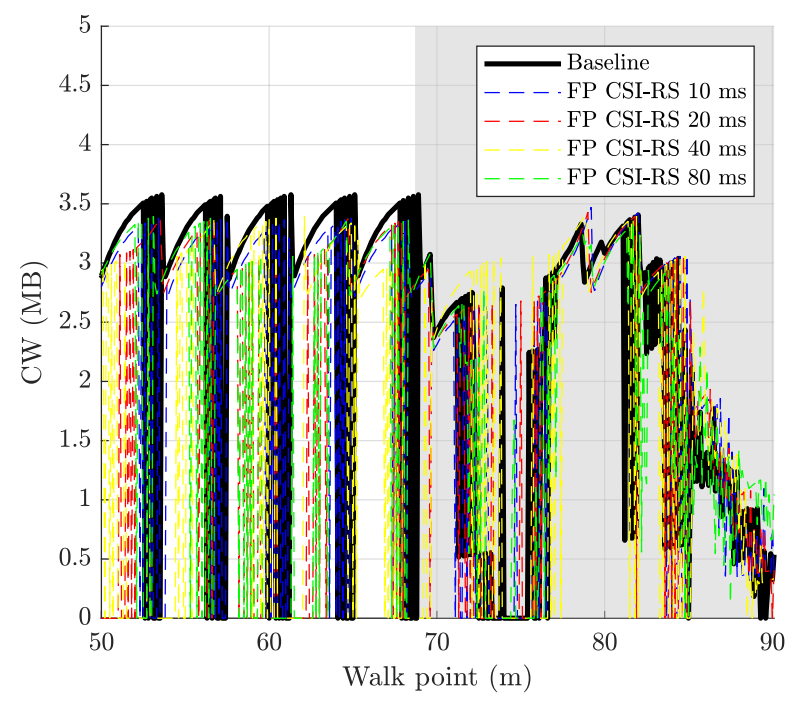

(a) Congestion Window.

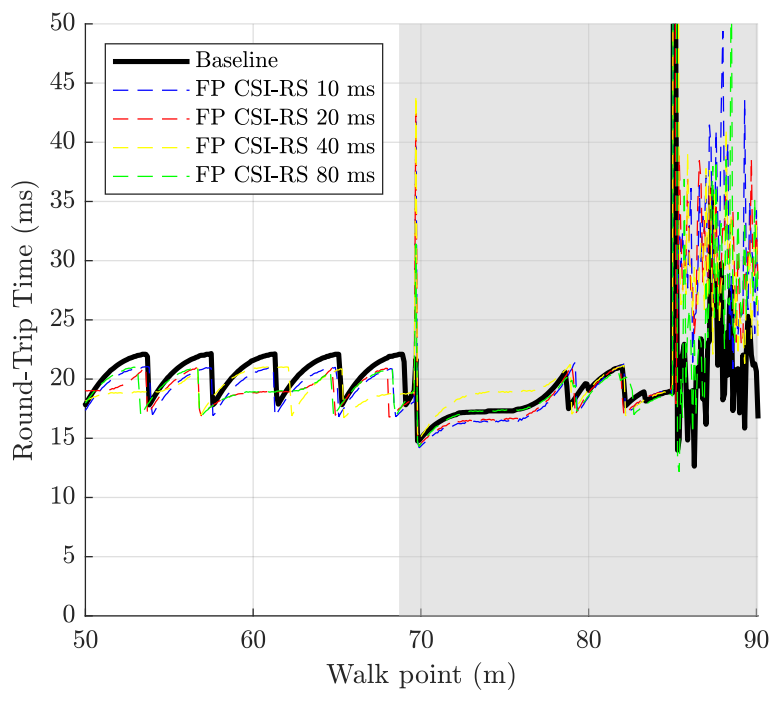

(b) Round Trip Time.

Figure 6.8: TCP statistics in inverse fingerprinting beam tracking at pedestrian speed with the long SS burst period. 
Table 6.3: Average throughput experienced in the whole UE walk, LoS and NLoS areas at pedestrian speed for an SS burst period of $20 \mathrm{~ms}$.

\begin{tabular}{|l|r|r|r|r|}
\hline \multirow{2}{*}{ Strategy } & \multicolumn{2}{|c|}{$\begin{array}{c}\text { CSI-RS } \\
\text { period (ms) }\end{array}$} & \multicolumn{3}{|c|}{ Avg. Throughput (Mbps) } \\
\cline { 3 - 5 } & - & 1243.38 & 1294.29 & 1080.69 \\
\hline \hline Baseline & - & 1220.08 & 1278.61 & 1033.07 \\
\hline \hline SSB-only & 10 & 1230.70 & 1279.50 & 1074.76 \\
\hline \hline \multirow{3}{*}{ Fingerprinting } & 20 & 1234.49 & 1278.07 & 1095.24 \\
\cline { 2 - 5 } & 40 & 1236.87 & 1278.10 & 1105.12 \\
\cline { 2 - 5 } & 80 & 1230.35 & 1278.45 & 1076.68 \\
\hline \hline \multirow{3}{*}{ Alt.A } & 10 & 1237.40 & 1279.50 & 1102.87 \\
\cline { 2 - 5 } & 20 & 1234.61 & 1278.07 & 1095.74 \\
\cline { 2 - 5 } & 40 & 1233.52 & 1278.10 & 1091.08 \\
\cline { 2 - 5 } & 80 & 1234.91 & 1278.45 & 1095.78 \\
\hline \multirow{3}{*}{ Alt.B } & 10 & 1232.20 & 1275.51 & 1093.81 \\
\cline { 2 - 5 } & 20 & 1175.89 & 1275.68 & 916.19 \\
\cline { 2 - 5 } & 40 & 1234.81 & 1277.15 & 1099.49 \\
\cline { 2 - 5 } & 80 & 1233.89 & 1278.02 & 1092.88 \\
\hline
\end{tabular}

there is no difference in the overhead in the selection of the CSI-RS period, as it can be derived from Table 2.4, Table 5.7 and Table 5.8. The small differences within same CSI-RS and SS burst periods is a side effect of TCP managing the congestion window on each situation.

\section{Urban micro scenario at vehicular speed}

This subsection continues the previous analysis for the vehicular mobility case in the UMi scenario. The evolution of the beam ids for an SS burst period of $160 \mathrm{~ms}$ are plotted in Figure 6.9. Figure 6.9(a) illustrates the evolution of the gNB beams in azimuth and elevation planes and Figure 6.9(b) represents the evolution of the UE beams. From a visual inspection, inverse fingerprinting beam tracking also achieves good beam alignment at vehicular speed.

Table 6.5 lists the beam alignment ratio and BGL for inverse fingerprinting, Alternative A and Alternative B beam tracking with a SS burst period of $20 \mathrm{~ms}$. Similarly, Table 6.6 provides the same performance metrics obtained with the longest SS burst period. Both tables confirm the impression from the visual inspection of Figure 6.9, which is that the beam alignment ratio is high, with a minimum coincidence probability of $80 \%$ with the smallest CSI-RS periodicity. 

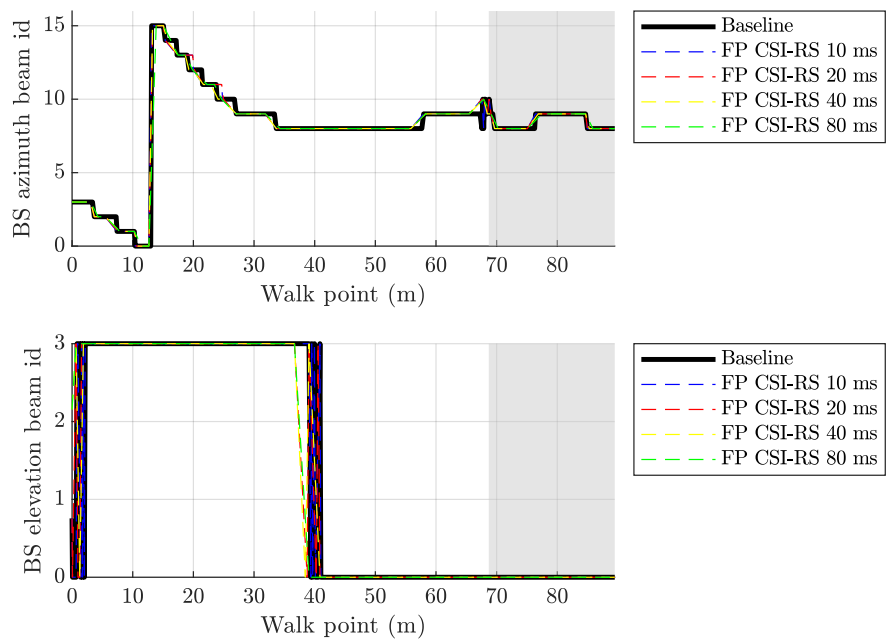

(a) gNB beam id.
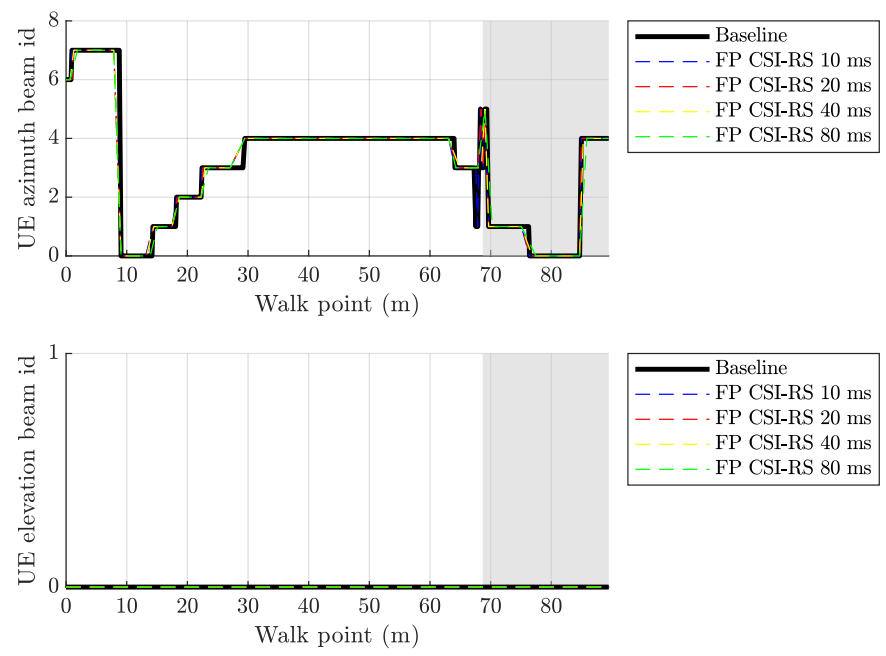

(b) UE beam id.

Figure 6.9: Selected beam ids over time at vehicular speed. 
Table 6.4: Average throughput experienced in the whole UE walk, LoS and NLoS areas at pedestrian speed for an SS burst period of $160 \mathrm{~ms}$.

\begin{tabular}{|l|r|r|r|r|}
\hline \multirow{2}{*}{ Strategy } & \multicolumn{2}{|c|}{$\begin{array}{c}\text { CSI-RS } \\
\text { period (ms) }\end{array}$} & \multicolumn{3}{|c|}{ Avg Throughput (Mbps) } \\
\cline { 2 - 5 } & - & 1243.38 & 1294.29 & 1080.69 \\
\hline \hline Baseline & - & 1203.72 & 1292.73 & 919.31 \\
\hline \hline SSB-only & 10 & 1245.27 & 1291.43 & 1101.85 \\
\hline \hline \multirow{4}{*}{ Fingerprinting } & 20 & 1247.49 & 1291.40 & 1107.17 \\
\cline { 2 - 5 } & 40 & 1246.06 & 1292.11 & 1098.92 \\
\cline { 2 - 5 } & 80 & 1244.48 & 1292.37 & 1091.47 \\
\hline \hline \multirow{4}{*}{ Alt.A } & 10 & 1242.14 & 1290.10 & 1088.92 \\
\cline { 2 - 5 } & 20 & 1246.03 & 1291.40 & 1101.07 \\
\hline \hline \multirow{4}{*}{ Alt.B } & 40 & 1247.57 & 1292.11 & 1105.23 \\
\cline { 2 - 5 } & 80 & 1240.35 & 1292.37 & 1074.14 \\
\cline { 2 - 5 } & 10 & 1242.49 & 1286.14 & 1103.04 \\
\cline { 2 - 5 } & 20 & 1246.47 & 1289.42 & 1109.25 \\
\cline { 2 - 5 } & 40 & 1242.87 & 1291.10 & 1088.74 \\
\cline { 2 - 5 } & 80 & 1243.77 & 1291.88 & 1090.04 \\
\hline
\end{tabular}

The BGL at vehicular speed is certainly larger than the pedestrian case due to the fact that the distance covered in beam misalignment is proportional to the user speed.

Figure 6.10(a) plots the curves of the experienced SINR in dB along the user walk time. As the CSI-RS period is increased, so are the beam misalignment areas. This becomes evident from a visual comparison with the baseline curve. This results in stronger SINR reductions. Despite experiencing a maximum reduction of approximately $25 \mathrm{~dB}$ in the LoS area, the SINR is still over $55 \mathrm{~dB}$. Fortunately, this SINR is sufficient to maintain the stationary throughput rate as it can be seen in Figure 6.10(b). In the transition to NLoS, the SINR may get as little as $10 \mathrm{~dB}$, and then it fluctuates between $40 \mathrm{~dB}$ and $30 \mathrm{~dB}$. As a result, the throughput recovers its stationary value. However, different CSI-RS periods $20 \mathrm{~ms}, 40 \mathrm{~ms}$ and $80 \mathrm{~ms}$ experience different throughput reductions and recovery times. As already mentioned, this recovery time is determined by the TCP congestion window control.

Table 6.7 and Table 6.8 provide the average throughput at vehicular speed for the SS burst periods of $20 \mathrm{~ms}$ and $160 \mathrm{~ms}$, respectively. Results are given for the whole UE walk, LoS and NLoS. Both tables contain the average values of the baseline case, SSB beam tracking and the beam tracking Alternative A 
Table 6.5: Beam alignment ratio and BGL at vehicular speed assuming an SS burst period of $20 \mathrm{~ms}$.

\begin{tabular}{|c|c|c|c|c|c|}
\hline \multirow{2}{*}{ Strategy } & \multirow{2}{*}{$\begin{array}{c}\text { CSI-RS } \\
\text { period }(\mathrm{ms})\end{array}$} & \multicolumn{3}{|c|}{$\mathbf{R}_{\text {alignment }}$} & \multirow{2}{*}{$\begin{array}{l}\text { BGL } \\
(\mathrm{dB})\end{array}$} \\
\hline & & Pair & gNB & $\mathbf{U E}$ & \\
\hline SSB-only & - & 0.41 & 0.49 & 0.70 & 3.31 \\
\hline \multirow{4}{*}{ Fingerprinting } & 10 & 0.81 & 0.81 & 0.99 & 1.64 \\
\hline & 20 & 0.79 & 0.80 & 0.98 & 1.69 \\
\hline & 40 & 0.76 & 0.78 & 0.97 & 1.91 \\
\hline & 80 & 0.70 & 0.73 & 0.94 & 2.38 \\
\hline \multirow{4}{*}{ Alt.A } & 10 & 0.80 & 0.81 & 0.98 & 1.87 \\
\hline & 20 & 0.78 & 0.80 & 0.97 & 2.06 \\
\hline & 40 & 0.74 & 0.78 & 0.95 & 2.19 \\
\hline & 80 & 0.68 & 0.73 & 0.92 & 2.66 \\
\hline \multirow{4}{*}{ Alt.B } & 10 & 0.81 & 0.81 & 0.99 & 1.64 \\
\hline & 20 & 0.79 & 0.80 & 0.98 & 1.69 \\
\hline & 40 & 0.76 & 0.78 & 0.97 & 1.91 \\
\hline & 80 & 0.70 & 0.73 & 0.94 & 2.38 \\
\hline
\end{tabular}

Table 6.6: Beam alignment ratio and BGL at vehicular speed assuming an SS burst period of $160 \mathrm{~ms}$.

\begin{tabular}{|c|c|c|c|c|c|}
\hline \multirow{2}{*}{ Strategy } & \multirow{2}{*}{$\begin{array}{c}\text { CSI-RS } \\
\text { period }(\mathrm{ms})\end{array}$} & \multicolumn{3}{|c|}{$\mathbf{R}_{\text {alignment }}$} & \multirow{2}{*}{$\begin{array}{l}\text { BGL } \\
(\mathrm{dB})\end{array}$} \\
\hline & & Pair & gNB & UE & \\
\hline SSB-only & - & 0.13 & 0.25 & 0.32 & 8.15 \\
\hline \multirow{4}{*}{ Fingerprinting } & 10 & 0.80 & 0.80 & 0.99 & 1.66 \\
\hline & 20 & 0.78 & 0.79 & 0.98 & 1.86 \\
\hline & $\overline{40}$ & 0.76 & 0.78 & 0.97 & 2.11 \\
\hline & 80 & 0.70 & 0.73 & 0.94 & 2.45 \\
\hline \multirow{4}{*}{ Alt.A } & 10 & 0.80 & 0.81 & 0.98 & 1.83 \\
\hline & 20 & 0.78 & 0.80 & 0.97 & 2.15 \\
\hline & 40 & 0.73 & 0.78 & 0.94 & 2.69 \\
\hline & 80 & 0.65 & 0.73 & 0.88 & 3.28 \\
\hline \multirow{4}{*}{ Alt.B } & 10 & 0.81 & 0.81 & 0.99 & 1.57 \\
\hline & 20 & 0.79 & 0.80 & 0.98 & 1.77 \\
\hline & 40 & 0.76 & 0.78 & 0.97 & 2.12 \\
\hline & 80 & 0.70 & 0.73 & 0.94 & 2.45 \\
\hline
\end{tabular}




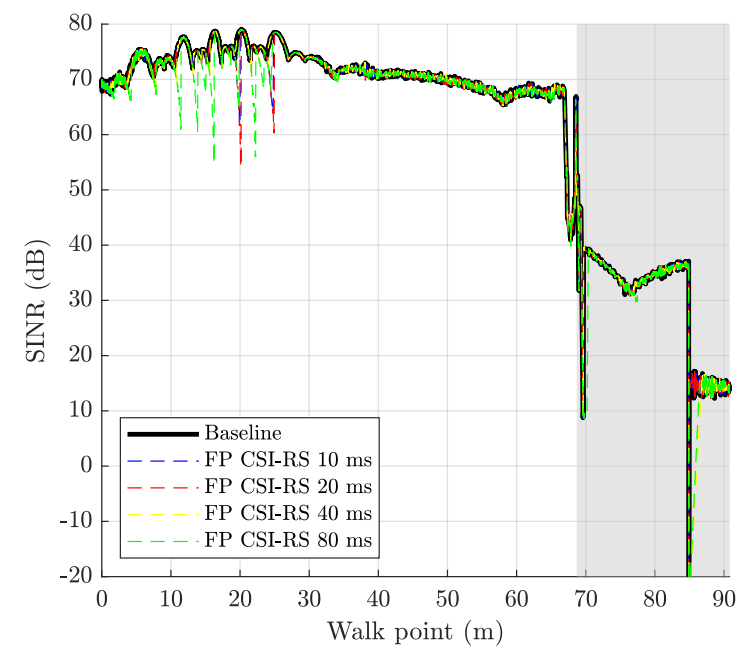

(a) SINR.

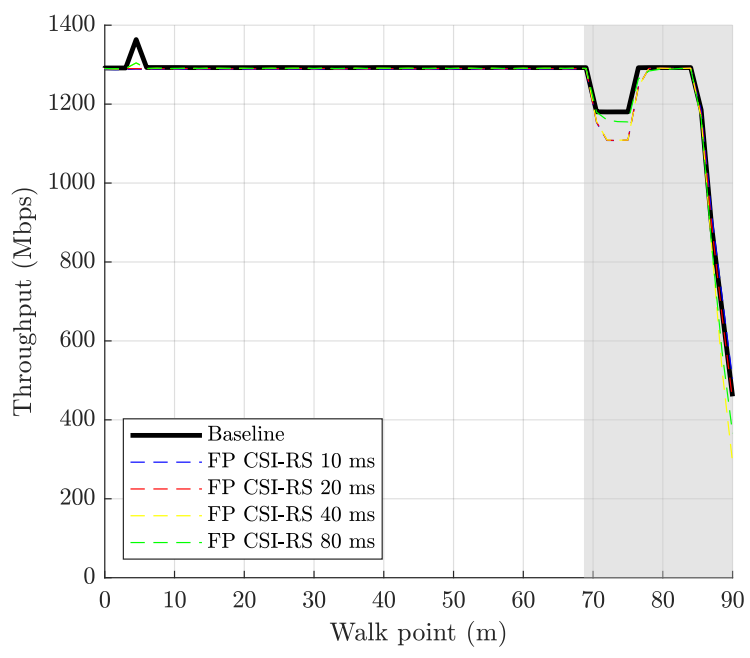

(b) User throughput.

Figure 6.10: Experienced SINR and user throughput with inverse fingerprinting beam tracking at vehicular speed. 
Table 6.7: Average throughput experienced in the whole UE walk, only-LoS and only-NLoS points for an SS burst period of $20 \mathrm{~ms}$ at vehicular speed.

\begin{tabular}{|l|r|r|r|r|}
\hline \multirow{2}{*}{ Strategy } & \multicolumn{2}{|c|}{$\begin{array}{c}\text { CSI-RS } \\
\text { period (ms) }\end{array}$} & \multicolumn{3}{|c|}{ Avg. Throughput (Mbps) } \\
\cline { 3 - 5 } & - & All & LoS & NLoS \\
\hline \hline Baseline & - & 1169.23 & 1294.07 & 1132.04 \\
\hline \hline SSB-only & 10 & 1244.12 & 1278.10 & 1139.89 \\
\hline \hline \multirow{3}{*}{ Fingerprinting } & 20 & 1241.98 & 1276.11 & 1137.32 \\
\cline { 2 - 5 } & 40 & 1237.17 & 1276.12 & 1117.72 \\
\cline { 2 - 5 } & 80 & 1232.24 & 1277.00 & 1094.99 \\
\hline \hline \multirow{3}{*}{ Alt.A } & 10 & 1239.31 & 1278.10 & 1120.33 \\
\cline { 2 - 5 } & 20 & 1229.75 & 1276.11 & 1087.58 \\
\hline \hline \multirow{3}{*}{ Alt.B } & 40 & 1237.48 & 1276.12 & 1118.97 \\
\cline { 2 - 5 } & 80 & 1216.94 & 1277.00 & 1032.78 \\
\cline { 2 - 5 } & 10 & 1242.03 & 1274.18 & 1143.43 \\
\cline { 2 - 5 } & 20 & 1238.24 & 1274.17 & 1128.03 \\
\cline { 2 - 5 } & 40 & 1233.80 & 1275.17 & 1106.93 \\
\cline { 2 - 5 } & 80 & 1230.64 & 1275.99 & 1091.56 \\
\hline
\end{tabular}

and Alternative B. Throughput values are averaged out in the whole UE walk and LoS and NLoS areas. Serving as reference, both tables contain the results from the baseline and the SSB beam tracking at the corresponding SS burst period.

At vehicular speed, the inverse fingerprinting beam tracking provides similar throughput values compared to Alternative A and Alternative B, with subtle differences.

\subsubsection{Simulation results with erroneous measurements}

This subsection discusses the effect that the errors in the determination of the orientation and location of the user device have on the beam tracking.

For the evaluation of the impact of the orientation error in the inverse fingerprinting beam tracking, this Thesis assumes that the UE is equipped with the low-cost electronic orientation device discussed in Section 6.1.2, which presents a maximum absolute orientation error of $3^{\circ}$. Accordingly, the orientation error is modelled as a uniform distribution taking values between $-3^{\circ}$ and $3^{\circ}$.

In this evaluation, the UE location procedure is also noisy and provides erroneous location information. The location error defines a circumference of 
Table 6.8: Average throughput experienced in the whole UE walk, LoS and NLoS areas for an SS burst periodicity of $160 \mathrm{~ms}$ at vehicular speed.

\begin{tabular}{|l|r|r|r|r|}
\hline \multirow{2}{*}{ Strategy } & \multicolumn{2}{|c|}{$\begin{array}{c}\text { CSI-RS } \\
\text { period (ms) }\end{array}$} & \multicolumn{3}{|c|}{ Avg Throughput (Mbps) } \\
\cline { 2 - 5 } & - & \multicolumn{1}{c|}{ All } & LoS & NLoS \\
\hline \hline Baseline & - & 710.01 & 843.55 & 300.50 \\
\hline \hline SSB-only & 10 & 1246.77 & 1288.42 & 1119.06 \\
\hline \hline \multirow{4}{*}{ Fingerprinting } & 20 & 1245.53 & 1289.78 & 1109.82 \\
\cline { 2 - 5 } & 40 & 1240.45 & 1290.69 & 1086.38 \\
\cline { 2 - 5 } & 80 & 1245.57 & 1291.04 & 1106.12 \\
\hline \hline \multirow{4}{*}{ Alt.A } & 10 & 1240.57 & 1288.42 & 1093.83 \\
\cline { 2 - 5 } & 20 & 1242.08 & 1289.78 & 1095.78 \\
\hline \hline \multirow{4}{*}{ Alt.B } & 40 & 1242.98 & 1290.69 & 1096.67 \\
\cline { 2 - 5 } & 80 & 1228.70 & 1291.04 & 1037.54 \\
\cline { 2 - 5 } & 10 & 1249.63 & 1284.51 & 1142.68 \\
\cline { 2 - 5 } & 20 & 1248.82 & 1287.82 & 1129.22 \\
\cline { 2 - 5 } & 40 & 1247.30 & 1289.74 & 1117.16 \\
\cline { 2 - 5 } & 80 & 1243.94 & 1290.28 & 1101.86 \\
\cline { 2 - 5 } & & & & \\
\hline
\end{tabular}

uncertainty, with the UE location in its centre, with a radius $r_{\text {loc_error }}$ set to the distance between the real location of the user and the maximum deviation in the estimated location. This Thesis assumes that the location error is uniformly distributed within the circumference of uncertainty. The value for $r_{\text {loc_error }}$ in this Thesis is equal to the maximum GPS error value of 7.8 metres motivated in Section 6.1.2.

Unless otherwise stated, the results shown for the inverse fingerprinting beam tracking with measurement errors assume an SS burst period of $160 \mathrm{~ms}$ and a CSI-RS period of $10 \mathrm{~ms}$.

\section{Urban micro scenario at pedestrian speed}

Figure 6.11 illustrates the SINR evolution in the UMi walk with different location errors. A location error of 0 metres for the beam tracking performance in the error-free simulations for the same beam tracking configuration. As it can be seen, SINR experiences large fluctuations in the initial 40 metres. This fluctuations are more significant as the location error increases, as the curves for error values of 1.5 metres, 2 metres, 2.5 metres or 3 metres indicate. This is due to the fact that the UE is located very close to the gNB and, there- 


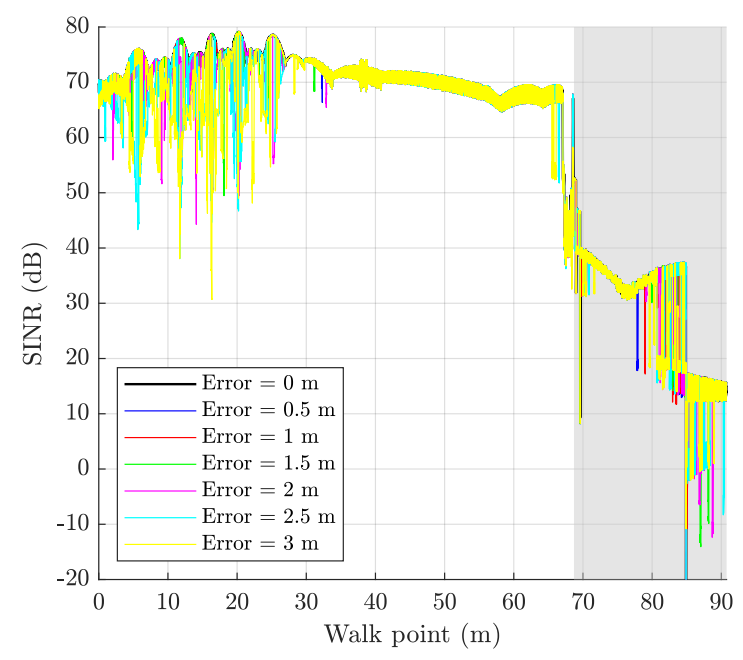

Figure 6.11: SINR levels for different location and orientation errors in UMi walk at pedestrian speed.

fore, the beam prints are smaller. As the UE walks away from the BS, the beam prints areas become larger and the location error effect is reduced. This fact, and having more gNB beams than UE beams, are behind the larger beam alignment ratio of the UE beams than the gNB beams in Table 6.9.

The NLoS area presents SINR oscillations in the last 12 metres of the simulation. The reduced SINR in the NLoS area makes it more sensitive to location errors since their value may fall below $20 \mathrm{~dB}$, as occurred for location errors as small as 1 metre.

Profound SINR oscillations in NLoS negatively influence on the user throughput in the last 12 metres of the user walk, as it can be seen in Figure 6.12. In this situation, the experienced throughput rapidly fluctuates between $50 \mathrm{Mbps}$ and $780 \mathrm{Mbps}$.

Table 6.9 summarises the beam tracking performance indicators, naming beam alignment ratio and BGL, obtained with the inverse fingerprinting beam tracking for different location errors. From the results obtained with different location errors at the UMi walk, the beam tracking performance worsens as the location error increases. But gNB beam alignment degrades more severely than UE beam alignment. This is motivated by the larger number of gNB beams than the UE beams considered in system model of this Thesis. In addition, 


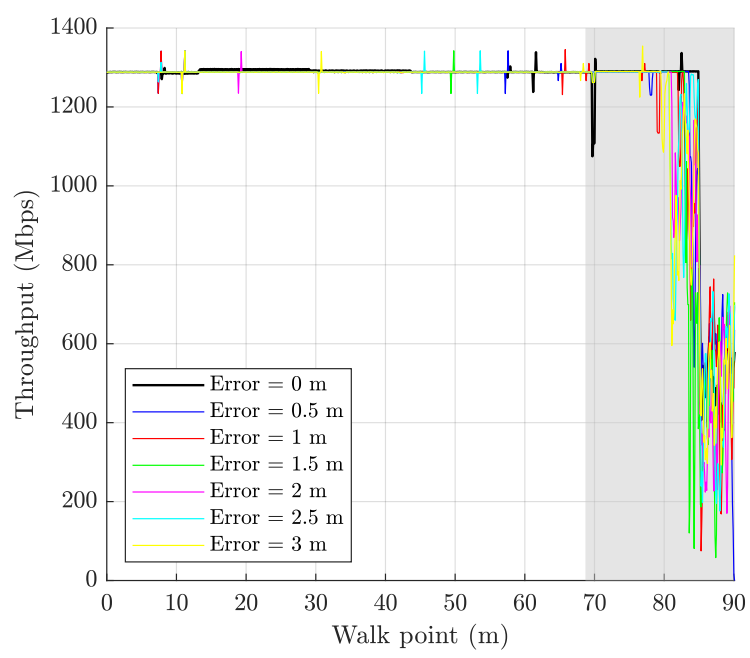

Figure 6.12: User throughput evolution for different location and orientation errors in UMi walk at pedestrian speed.

gNB beams are narrower than UE beams and more likely to change the beam id in case of error in the location.

Since the throughput is not penalised by the strong SINR fluctuations, especially in LoS, it is assumed that the location error penalises the beam tracking ability if the BGL decreases a threshold $b g l_{\text {threshold }}$ value of $3 \mathrm{~dB}$. Accordingly, the beam pair alignment ratio is significantly penalised with a minimum error of 2 metres, with a $18 \%$ reduction of the beam alignment ratio and an increase of $3.15 \mathrm{~dB}$ in the BGL value with respect to the result obtained in the error-free system. With a location error of 7 metres, the BGL experiences a $11.53 \mathrm{~dB}$ increase, the beam pair alignment achieves a $54 \%$, the gNB beam alignment is $63 \%$ and the UE beam alignment is $77 \%$ with a location.

In general, as the location error increases, the beam alignment ratio reduces and the BGL increases. Such reduction of the beam gain may lead to data rate discontinuations in situation with low SINR, which can be the case in multi-user scenarios.

\section{Urban micro scenario at vehicular speed}

For the vehicular case, Table 6.10 summarises the performance of the inverse fingerprinting beam tracking for the location error values evaluated in the 
Table 6.9: Beam alignment ratio and BGL obtained in the UMi walk at pedestrian speed by an inverse fingerpriting beam tracking with different location error values and a maximum orientation deviation of $3^{\circ}$. The beam tracking configuration consists of an SS burst period of $160 \mathrm{~ms}$ and a CSI-RS period of $10 \mathrm{~ms}$.

\begin{tabular}{|c|c|c|c|c|}
\hline \multirow{2}{*}{$\begin{array}{l}\text { Location } \\
\text { error }(m)\end{array}$} & \multicolumn{3}{|c|}{ Beam alignment } & \multirow{2}{*}{$\begin{array}{l}\text { BGL } \\
\text { (dB) }\end{array}$} \\
\hline & Pair & gNB & UE & \\
\hline 0 & 0.96 & 0.96 & 0.99 & 4.34 \\
\hline 0.5 & 0.95 & 0.95 & 0.98 & 4.42 \\
\hline 1 & 0.91 & 0.92 & 0.98 & 5.36 \\
\hline 1.5 & 0.88 & 0.90 & 0.97 & 5.06 \\
\hline 2 & 0.78 & 0.82 & 0.93 & 7.49 \\
\hline 2.5 & 0.75 & 0.82 & 0.91 & 9.07 \\
\hline 3 & 0.69 & 0.78 & 0.86 & 9.25 \\
\hline 4 & 0.63 & 0.71 & 0.85 & 11.54 \\
\hline 5 & 0.58 & 0.68 & 0.80 & 12.01 \\
\hline 6 & 0.59 & 0.65 & 0.82 & 14.44 \\
\hline 7 & 0.54 & 0.63 & 0.77 & 15.87 \\
\hline
\end{tabular}

pedestrian speed case. Assuming the same $b g l_{\text {threshold }}$ value of $3 \mathrm{~dB}$ used in the pedestrian speed case, the beam tracking performance at vehicular speed is penalised with a location error of 3 metres, with a $33 \%$ reduction of the beam alignment ratio and an increase of $3.20 \mathrm{~dB}$ in the BGL value with respect to the result obtained in the error-free system. This beam tracking performance indicators are significantly better than the same comparison done in the pedestrian walk. Nevertheless, the beam tracking performance indicators with errors are worse in the vehicular speed than in the pedestrian case. As occurred in the pedestrian case, if the location error increases, the beam alignment ratio reduces and the BGL increases. Beam pair alignment falls below $50 \%$ for all the location errors, the gNB beam alignment is under $50 \%$ with a location error of 1 metres and the UE beam alignment is under $50 \%$ with a location error of 2 metres.

Figure 6.13 illustrates the SINR evolution in the UMi walk with different location errors. The SINR level experience oscillations in the initial 40 metres. As occurred in the pedestrian walk, the level differences are larger for larger location errors.

Figure 6.14 represents the user throughput evolution for different location and orientation errors. Despite of the large SINR value deviations in LoS, the user throughput is not negatively affected. The reason is that SINR is 
Table 6.10: Beam alignment ratio and BGL obtained in the UMi walk at vehicular speed by an inverse fingerpriting beam tracking with different location error values and a maximum orientation deviation of $3^{\circ}$.

\begin{tabular}{|c|c|c|c|c|}
\hline \multirow{2}{*}{$\begin{array}{l}\text { Location } \\
\text { error }(m)\end{array}$} & \multicolumn{3}{|c|}{ Beam alignment } & \multirow{2}{*}{$\begin{array}{l}\text { BGL } \\
(d B)\end{array}$} \\
\hline & Pair & gNB & UE & \\
\hline 0 & 0.80 & 0.68 & 0.86 & 1.66 \\
\hline 0.5 & 0.82 & 0.84 & 0.97 & 2.13 \\
\hline 1 & 0.81 & 0.82 & 0.98 & 2.35 \\
\hline 1.5 & 0.77 & 0.80 & 0.96 & 2.38 \\
\hline 2 & 0.74 & 0.77 & 0.94 & 2.82 \\
\hline 2.5 & 0.71 & 0.76 & 0.93 & 4.31 \\
\hline 3 & 0.47 & 0.68 & 0.59 & 3.86 \\
\hline 4 & 0.43 & 0.63 & 0.58 & 4.57 \\
\hline 5 & 0.43 & 0.64 & 0.56 & 4.67 \\
\hline 6 & 0.45 & 0.68 & 0.61 & 8.36 \\
\hline 7 & 0.38 & 0.57 & 0.57 & 11.59 \\
\hline
\end{tabular}

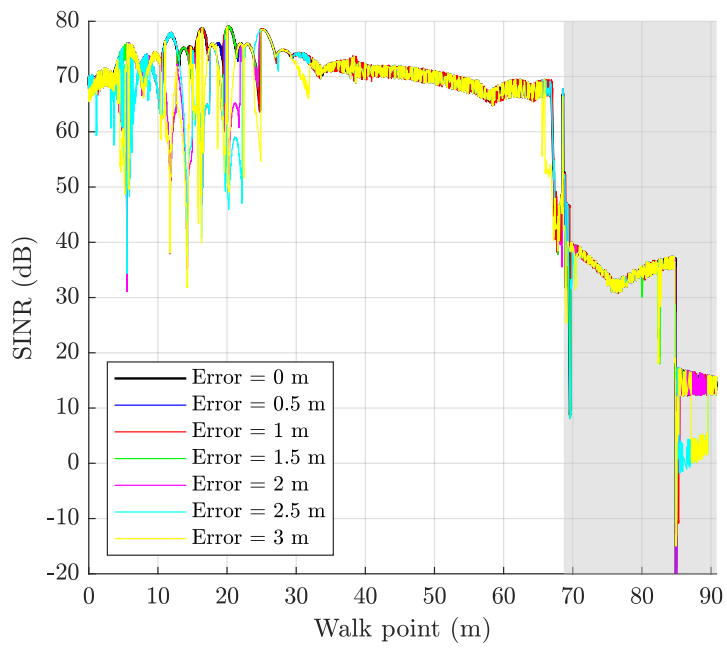

Figure 6.13: SINR levels for different location and orientation errors in UMi walk at vehicular speed. 


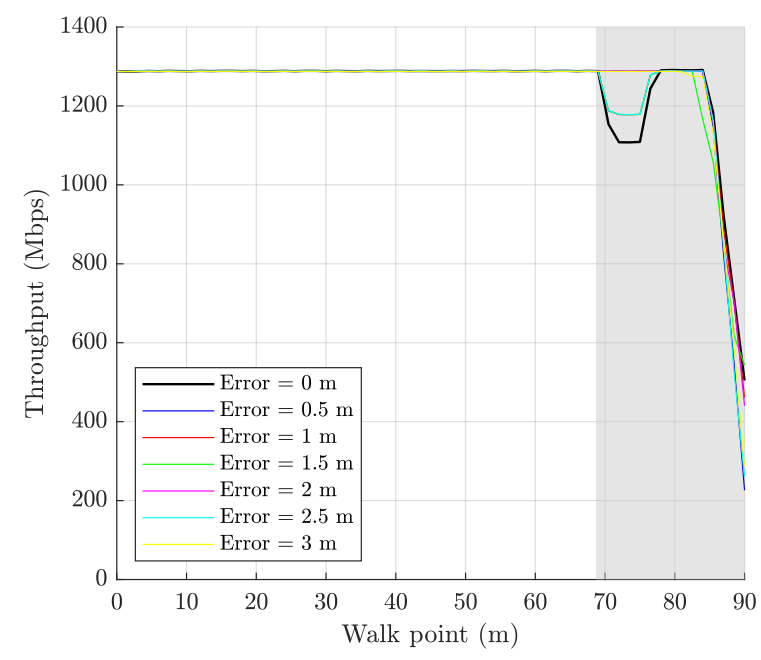

Figure 6.14: User throughput evolution for different location and orientation errors in UMi walk at vehicular speed.

maintained in good levels over $30 \mathrm{~dB}$ In the NLoS region, the SINR reduction at walk point 85 metres originates the faster throughput reduction for location errors larger than 2 metres.

\subsubsection{Beam pair determination based on user location}

The inverse fingerprinting database allows a diversity of information and formats. This includes the list of pair of beams to monitor with beam measurements of CSI-RS resources. In typical fingerprinting applications for cellular user location, the network trusts in the information contained in the database to locate the user. With the same philosophy, this study aims at determining the impact of the location error in an inverse fingerprinting beam tracking strategy which strictly follows the optimal beam pair reported by the database. In this case, no CSI-RS resource is allocated nor any CSI-RS beam measurement is performed. And the UE connects with the serving gNB through the beams indicated by the database according to the estimated user location.

This kind of beam determination using an inverse fingerprinting is of interest in deployments where a gNB has a large number of simultaneous connections. In such case, the overhead due to CSI-RS scales with the number of connections, 
Table 6.11: Beam alignment ratio and BGL obtained in the UMi walk at pedestrian speed for the inverse fingerpriting beam determination and beam tracking with different location error values and a maximum orientation deviation of $3^{\circ}$.

\begin{tabular}{|c|c|c|c|c|c|c|c|c|}
\hline \multirow{3}{*}{$\begin{array}{c}\text { Loc. } \\
\text { error }(\mathbf{m})\end{array}$} & \multicolumn{4}{|c|}{ Beam determination } & \multicolumn{4}{|c|}{ Beam tracking } \\
\hline & \multicolumn{3}{|c|}{ Beam alignment } & \multirow{2}{*}{$\begin{array}{l}\text { BGL } \\
\text { (dB) }\end{array}$} & \multicolumn{3}{|c|}{ Beam alignment } & \multirow{2}{*}{$\begin{array}{l}\text { BGL } \\
\text { (dB) }\end{array}$} \\
\hline & Pair & gNB & UE & & Pair & gNB & UE & \\
\hline $\begin{array}{ll}0 \\
\end{array}$ & $\overline{0.96}$ & 0.96 & $\overline{0.99}$ & 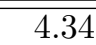 & $\overline{0.96}$ & $\overline{0.96}$ & 0.99 & 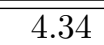 \\
\hline 0.5 & 0.52 & 0.62 & 0.84 & 5.93 & 0.95 & 0.95 & 0.98 & 4.42 \\
\hline 1 & 0.45 & 0.54 & 0.79 & 8.80 & 0.91 & 0.92 & 0.98 & 5.36 \\
\hline 1.5 & 0.46 & 0.54 & 0.75 & 11.24 & 0.88 & 0.90 & 0.97 & 5.06 \\
\hline 2 & 0.42 & 0.51 & 0.6 & 12.90 & 0.78 & 0. & 0.93 & 7.49 \\
\hline 2.5 & 0.38 & 0.47 & 0.65 & 13.89 & 0.75 & 0.8 & 0.91 & 9.07 \\
\hline 3 & 0.33 & 0.42 & 0.59 & 16.17 & 0.69 & 0.7 & 0.86 & 9.25 \\
\hline 4 & 0.30 & 0.37 & 0.52 & 20.09 & 0.63 & 0.7 & 0.85 & 11.54 \\
\hline 5 & 0.26 & 0.31 & 0.5 & 20.49 & 0.58 & 0. & 0.80 & 12.01 \\
\hline 6 & 0.20 & 0.27 & 0.4 & 21.62 & 0.59 & 0. & 0.82 & 14.44 \\
\hline 7 & 0.20 & 0.29 & 0.43 & 22.98 & 0.54 & 0.63 & 0.77 & 15.87 \\
\hline
\end{tabular}

assuming that all the users are always allocated the same number of CSI-RS values.

Table 6.11 contains the beam alignment ratio and BGL of the inverse fingerprinting beam determination for the pedestrian speed. These results were obtained considering an error-free data collection campaign. The results in the table are compared to the values obtained with the inverse fingerprinting beam tracking. Note that the difference between the two strategies is the number of CSI-RS resources, and the number of beam pairs measured, involved: for beam determination no beam measurements are performed and no CSI-RS are allocated.

As it can be seen in Table 6.11, beam determination is more sensitive to location errors than beam tracking. In this case, monitoring a candidate list of pairs of beams helps mitigating the effect of choosing a sub-optimal pair of beams due to the location error. A small location error of 0.5 metres reduces the beam alignment ability severely. As a consequence, the BGL is superior in the beam determination case.

The beam alignment sensitivity to errors of the inverse fingerprinting beam determination strategy is confirmed with a visual inspection SINR evolution in Figure 6.15. The oscillations in the initial 40 metres are more frequent and deeper. And this trend is also observed in the NLoS area. 


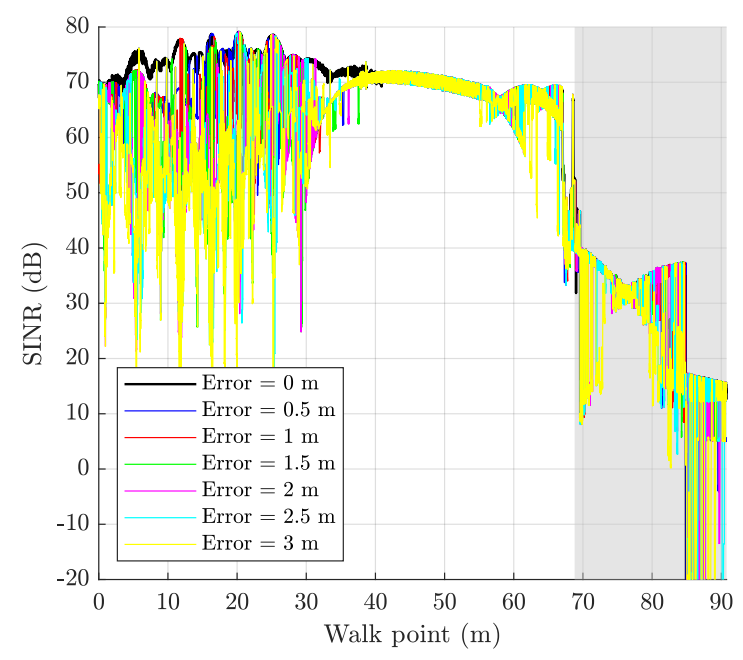

Figure 6.15: SINR levels for inverse fingerprinting beam determination with different location errors.

As a result of the large SINR value fluctuations, the experienced user throughput presents fluctuations in LoS, even at pedestrian speed, due to the strong beam misalignments with location errors of 1.5 metres, 2 metres, 2.5 metres, and 3 metres, as it can be seen in Figure 6.16.

In light of this results, the inverse fingerprinting beam determination is not recommended for dense urban networks due to the location errors in real-world network deployments.

\subsubsection{Conclusions on fingerprinting}

This section has introduced a beam tracking strategy based on contextual information provided by an inverse fingerprinting database. The database relates the reported UE location and orientation with the corresponding optimal pairs of beams. The beam tracking approach makes use of a maximum number of 20 CSI-RS resources to monitor the most promising combination of gNB beam and UE beam for the estimated location of the UE.

Assuming an error-free system, with precise information of the user location and the orientation, excellent beam tracking results can be obtained with any combination of SS burst period and CSI-RS period. 


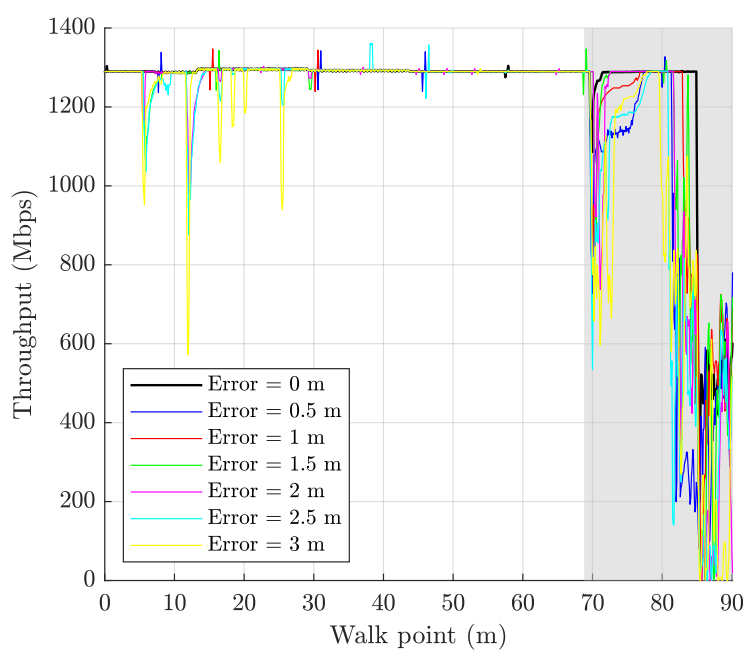

Figure 6.16: User throughput evolution for inverse fingerprinting beam determination with different location errors.

However, when assuming real-world conditions, the inverse fingerprinting beam tracking fails. Accelerometers and gyroscopes in typical smartphones are economic and yet provide acceptable device orientation estimations, with a maximum estimation error of $3^{\circ}$. For the urban scenarios evaluated in this Thesis, the range of orientation error values evaluated does not have a significant contribution to an erroneous beam determination. More precisely, UE beam id has a maximum error probability of $15 \%$ due to orientation errors, whereas the maximum probability of determining a wrong gNB beam is $1.5 \%$. On the other hand, errors in the user location clearly diminishes the beam tracking ability. By means of end-to-end network simulations with different location errors, it has been shown that inverse fingerprinting achieves a beam tracking ratio below 0.75 in urban scenarios with an estimation error of 3 metres for the pedestrian speed case and 2 metres for the vehicular speed case, and the degradation increases proportionally to the location error.

The inverse fingerprinting beam tracking is fundamentally based on the determination of the user location. However, cellular users are taking conscience of their data privacy and are more reluctant to share sensitive data like their position and tend to block the reporting of their location. In this scenario, alternative user location mechanisms must be found, and can be totally compatible with the inverse fingerprinting beam tracking. Independently of the location 
procedure used, its precision shall not exceed the location error threshold of 7.8 metres for urban scenarios evaluated in this Thesis. However, this level of precision may be compromised in street canyon scenarios as reported in $[113,115]$. Therefore, the viability of the inverse fingerprinting beam tracking depends on the adoption of mechanisms to improve the location accuracy in order to make the beam tracking less prone to misalignment situations due to location errors.

A real-world deployment would require that the database foresees erroneous measurements. However, the wide variety of types, complexities and manufacturers of the Fifth Generation (5G) devices, makes considering all kind of errors very challenging. In addition, for a good performance of the inverse fingerprinting beam tracking, the database should also consider all the possible blocking phenomena due to moving obstacles like vehicles. The joint complexity of constructing a database considering all possible blockages and erroneous measurements is clearly prohibitive.

Due to the large sensitivity to noisy measurements, beam determination is not recommended for urban scenarios according to the poor beam alignment performance and the consequent loss in SINR, which has led to significant throughput rate reductions in the evaluated scenario. But it is worth noting that this strong SINR loss may lead to temporal service discontinuation due to a loss of connectivity. For this reason, multiple CSI-RS resources should be allocated to monitor alternative beam pairs in an attempt of reducing the impact of the location error in the determination of the beam to use.

In conclusion, due to the complexity of the inverse fingerprinting database for beam tracking and its sensitivity to erroneous or noisy measurements in realistic conditions, which leads to a poorer beam tracking performance and service quality than the beam tracking strategies based on Reference Signal (RS) measurements introduced in Chapter 5, fingerprinting according to the mechanism presented in this Thesis is unfeasible for dense urban networks.

\subsection{Beam tracking with machine learning}

The second context-aware beam tracking strategy considered in this Thesis is using a Machine Learning (ML) model. Due to the complexity of the beam tracking problem in dynamic environments, ML models appear as a natural solution given its ability to learn trends and patterns from a prerecorded sets of data.

This Thesis presents the beam tracking problem as a multi-class classification problem. The designed ML model should be able to make predictions on the next optimal pair of beams to used based on the historical of certain input 
parameters or features. NNs have demonstrated excellent classification abilities in pattern recognition problems. Theoretically, an NN with a sufficiently large number of neurons can classify any type of data with an arbitrary accuracy. NNs are convenient for classification problems with complex or irregular decision boundaries over many variables. Accordingly, NN seems a promising technology to solve the beam tracking problem in challenging scenarios.

This section of the Thesis aims at providing a preliminary study of the viability of ML models for beam tracking. This section first introduces some fundamental data processing concepts which help understanding how to adequate raw data in order to successfully train the ML model. Then, the candidate ML model is presented and, then, a discussion on its beam tracking performance with different sets of data is given. The section finishes by drawing the main conclusions of this preliminary study.

\subsubsection{Data preprocessing}

There are many ML techniques to choose from in order to solve a particular problem. But without a large collection of good data realisations, the ML model will simply not work. Most of the times, working ML models experiment significant performance boosts by selecting the data wisely. Therefore, data preprocessing plays a significant role in the success of the learning model.

\section{Feature selection}

Feature selection is one of the key processes in ML [116]. The main premise of feature selection is finding among all features those which are not necessary to successfully train the ML network. A feature is irrelevant if its presence or not provides the same result in the ML network. A redundant feature is one which provides the same information as another feature. In other words, there is a strong correlation among features. As a result, the input data is simply replicated.

Removing irrelevant and redundant features has one direct implication which is the reduction of the data set, which brings a reduction in the time required to train the network. This time reduction is more accentuated when the data set is large.

Removing unnecessary features also helps understanding the underlying data model better. For instance, it helps identifying the features that are important for the model characterisation.

In addition, performing feature selection improves the accuracy of the predictors. By removing non-essential features, the noise induced to the system is reduced, so the probability to overfit the network to noise is reduced. 


\section{Feature scaling}

One classical data preprocessing step is feature scaling [117]. When having different features, each of them may have different magnitude orders or range values. In such situations, some machine learning algorithms may take long time converge, or maybe they will never do. The underlying reason of this undesired behaviour is simply a mathematical question. Typical classifiers used in ML use the Euclidean distance to determine the cost function. So, if one of the features has a large range of values, this feature will have a larger Euclidean distance and, as a result, a larger weight in the following iterations of the algorithm.

Therefore, the range of each independent feature should be normalised so that they contribute approximately proportionately to the final distance. This is true at least in the first iterations. As the ML network iterates the true contribution of each feature in the final decision will be discovered with sufficient training samples.

Feature scaling is more than recommended in ML algorithms using stochastic gradient descent. The convergence speed of the algorithm can be largely improved with feature scaling. Similarly, when applying feature scaling to a Support Vector Machine (SVM), the number of iterations required to find the support vectors can be dramatically shortened. Note that different results are obtained from a SVM when applying feature scaling or not, since ranges and distances are different.

This Thesis assumes min-max normalisation, also known as rescaling, as the feature scaling option. Min-max normalisation consists on changing the range of values of each feature so their possible values are in the range of $[0,1]$ or $[-1,1]$. The selection of one or the other range depends on the nature of the data. The general formula of min-max normalisation is:

$$
\mathbf{x}^{\prime}=\frac{\mathbf{x}-\min (\mathbf{x})}{\max (\mathbf{x})-\min (\mathbf{x})}
$$

where $\min (\mathbf{x})$ is the minimum value of the vector feature and $\max (\mathbf{x})$ is the maximum value of the feature vector.

\subsubsection{The Multi-Layer Perceptron (MLP)}

An MLP is a shallow, artificial neural network. It consists of a minimum number of 3 layers of nodes: an input layer, a hidden layer and an output layer. In this configuration of the network, the information is passed from the input layer to the hidden layer, and finally from the hidden layer to the output 
layer. This kind of NN models in which the information travels in one direction are referred to as feedforward networks.

$$
\mathbf{y}=\mathbf{w}^{T} \cdot \mathbf{x}+b=\sum_{i=0}^{n} w_{i} x_{i}+b
$$

Except of the input layer, the output of each perceptron in a MLP is transformed by a nonlinear function called the activation function $\Phi$ :

$$
\mathbf{y}=\Phi\left(\sum_{i=0}^{n} w_{i} x_{i}+b\right)
$$

Using the vectorial notation, Equation 6.3 can be written as:

$$
\mathbf{y}=\Phi\left(\mathbf{w}^{T} \mathbf{x}+b\right)
$$

The activation function can be an arbitrary continuous function. The classic activation function in MLP is the sigmoid function, also known as logistic function:

$$
\Phi_{\text {sigmoid }}(v)=\frac{1}{1+\exp (-v)}
$$

where $v$ is the output of the neuron. The sigmoid function presents some computation problems. With the later advancements in NNs and deep learning, sigmoid functions have been replaced by Rectifier Linear Unit (ReLU) functions [118] in different flavours:

$$
\Phi_{\mathrm{ReLU}}(v)= \begin{cases}0 & \text { if } v \leq 0 \\ v & \text { if } v>0\end{cases}
$$

The sigmoid and ReLU activation functions are illustrated in Figure 6.17. The selection of an activation function is not trivial and depends on the nature of the data being processed. The choice has an impact on the error of the prediction and also in the training speed. This being said, there is no one-fitsall activation function for all the classification problems.

The differential characteristic of a MLP is that perceptrons are fully connected, which means that each node in one layer connects with every node in the next layer. A MLP network with one hidden layer is illustrated in Figure 6.18.

The input layer has as many neurons as features in a one-to-one relationship. Despite of the nomenclature, the input layer does not perform any multiplication of the features; the neurons simply pass the values to the first hidden layer. 
CHAPTER 6. CONTEXTUAL BEAM TRACKING

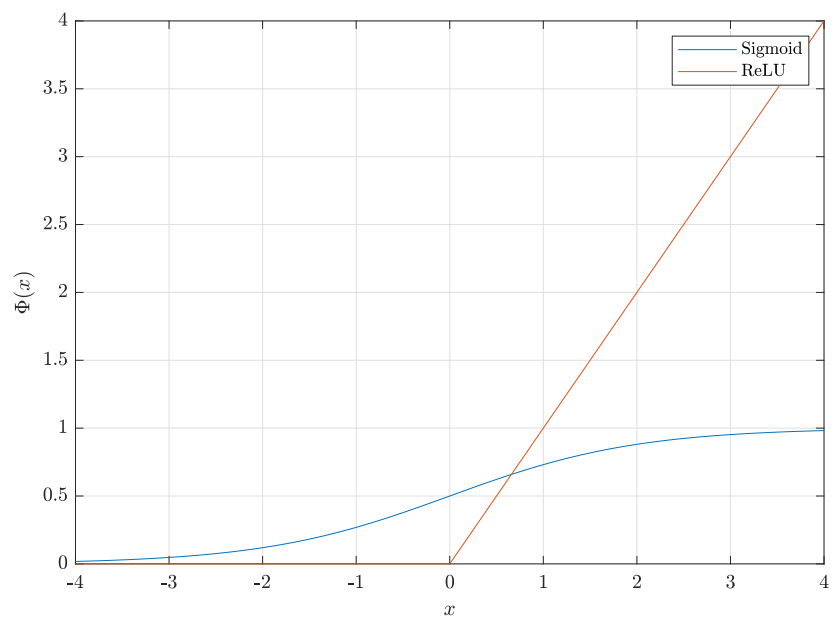

Figure 6.17: Response of the sigmoid and ReLU activation functions.

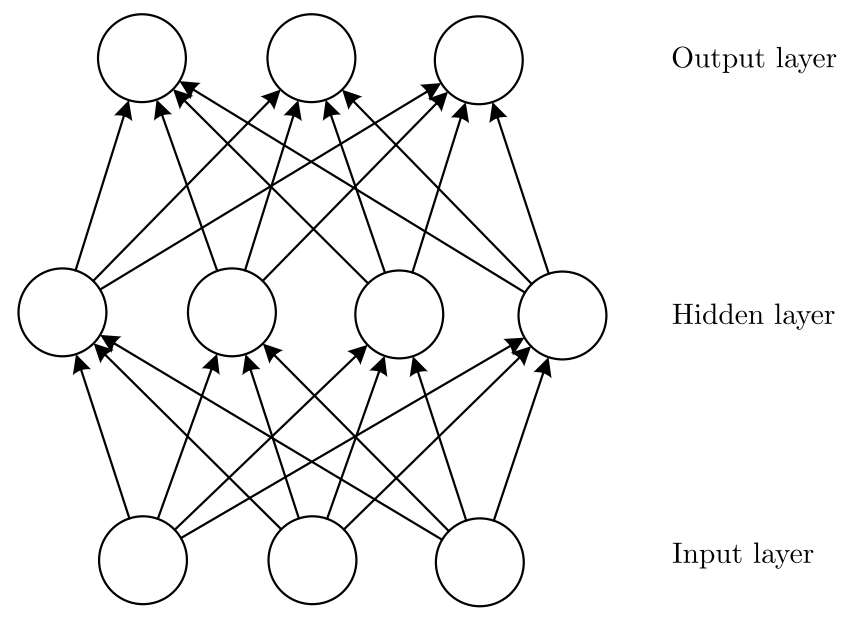

Figure 6.18: A MLP with one hidden layer. 
The computational power of the MLP resides in its hidden layers. Theoretically, they can approximate any continuous function. The number of hidden layers scales with the complexity of the function to approximate. The number of perceptrons or neurons per hidden layer is also a design decision and each layer can have different number of neurons.

Formally, a MLP as the one depicted in Figure 6.18 is a mathematical function $f: \mathbb{R}^{D} \rightarrow \mathbb{R}^{L}$ which takes $D$ real numbers as features and gets $L$ real numbers. The model function can be written as follows:

$$
y(x)=\Gamma\left(b^{(2)}+W^{(2)}\left(\Phi\left(b^{(1)}+W^{(1)} x\right)\right)\right)
$$

where $\mathbf{b}$ is the bias vector, $\mathbf{W}$ is the weight matrix and $\Gamma$ and $\Phi$ are activation functions of output and hidden layers, respectively. Note that the activation functions are given different nomenclatures. The output layer has a different activation function, the softmax function, which is used with multiclass outputs and takes two possible binary values: 0 or 1 . The superscripts indexes refer to a layer. The numbering starts with 1 being assigned to the layer which is closer to the input layer, and sequentially increases through the following layers until the output layer. In the example in Figure, superscript 1 denotes the hidden layer and superscript 2 refers to the output layer.

MLPs can be applied to supervised learning problems. Training a NN summarises in adjusting the weights and biases so the errors or differences between the values predicted by the model and the real results obtained from observations are minimised. This error is commonly quantified with Root Mean Squared Error (RMSE). The parameter adjustments are done using a backpropagation algorithm [119], a special case of the chain-rule of derivation. The details of the backpropagation and gradient descent methods are left out of the scope of this Thesis.

\subsubsection{An MLP model for beam tracking}

Adopting an ML model for beam tracking demands some previous considerations. First and foremost, it is important to understand that there are different hyperparameters involved in the success of a given ML model: the number of input features and their nature, the data normalisation used, the number of neurons, the number of hidden layers or the depth of the NN, the activation functions at the end of each layer, the NN model, etc. There is no standard procedure for determining the optimal configuration of a model for a specific application. Finding the optimal combination of hyperparameters is an iterative tuning procedure in which, from a starting model configuration, the objective metric is minimised, normally the RMSE or a variant [120]. 


\section{Data preparation}

The number of data sources involved in the beam tracking problem within the framework of a mmWave NR network is diverse to some extend. It is possible to categorise the nature of the potential information to feed a ML network for beam tracking:

1. Network information. This category includes all types of network-related information, which are empirically created from the real-time performance of the network. Examples of this category are measurement reports and Key Performance Indicator (KPI).

2. Contextual information. This category includes all information sources which are not inherent to the cellular network. In wireless systems, this kind of information source is also named out-of-band information or sideband information. In some applications, these external sources are used to complement or substitute the network information. A clear example of use of external information in cellular systems is using the GPS module in smartphones to provide location information.

The features fed into a ML model can be obtained from any type of the two data sources. A beam management framework in NR can surely count with beam information and measurement reports of SSB and CSI-RS, and the geolocation information of the UE obtained, from instance, from a GPS receiver embedded in the UE. In the beam management literature in mmWave, some researchers go further and adopt other external sources which are idealistic, or at least the information and accuracy level provided is hard to obtain in real situations. Such assumptions go from using error-free location information or knowing the location of users and obstacles like Klautau et al. in [71].

The fundamental problem of the ML approach for beam tracking is the lack of real measurements. Therefore, useful data collections shall be artificially obtained. These synthetic channel realisations must be formatted to be compatible with the system model defined in Section 3.1. Therefore, the reference data collection must provide the delay, attenuation, azimuth Angle of Departure (AoD), azimuth Angle of Arrival (AoA), elevation AoD, and elevation AoA for each ray arriving at the measurement location. The resulting beam gains must be converted to a 2 -dimensional beam map using the BS and UE codebooks defined in Section 3.3.

Next, it is necessary to obtain the temporal evolution of the beam pair selection of a moving user. This information will be obtained from the beam tracking Alternative B described in Section 5.3.1. Note that the adopted beam tracking strategy monitors beams which are immediately next to the optimal 
beams (with a beam distance d of 1 beam) and additional equally spaced beams on the azimuth domain. For each temporal simulation time, the beam pair evolution provided by the mentioned beam tracking strategy is obtained.

The obtained beam evolution will be used to train and evaluate the ML model. Typically, the whole data set is randomly split into train, validation and test sets. This Thesis assumes that train, validation and test data sets satisfy the following proportions:

- $70 \%$ of the data to train the network.

- $15 \%$ of the data for validation.

- $15 \%$ of the data for testing the network.

\section{The MLP model}

The MLP model takes the past $N$ beam measurement reports from the selected beam tracking strategy to predict which pair of beams to use next. To this aim, the MLP network takes a number of of input vectors of features $F$, naming transmit and receive beam ids, beam gains or SINR levels, user location, etc. So, the input data in the MLP can be seen as a matrix with $F \times N$ rows and $R$ columns, where $R$ is the number of data samples.

For the success in training the MLP model, it is vital that the feature values are normalised following the min-max normalisation described in Section 6.2.1. Also note that the feature vectors must have the same length $R$.

Since this is a multi-class classification problem, the network has as many outputs as classes. In the particular application for beam tracking, the number of outputs of the MLP model is the number of transmit beams multiplied by the number of receive beams, which is, according to the system model assumption in Section 5.1, 1024.

\section{Predicting beams from an external database}

Creating the high-precision realistic channel realisations described in Section 3.2.2 takes several hours. For this reason, an external data source is used. For the ML approach, in the first place, the MLP model is trained with the same data collection used in [71] for vehicular communications. The fundamental characteristics of this particular data set is that data is organised in 116 scenes, and each scene may contain information of 10 users in 50 channel realisations with a temporal resolution of $100 \mathrm{~ms}$ between each realisation, resulting in a simulation time of 5 seconds. The episodes include vehicles that are moving at unknown speeds, and some remain static during the simulations. In 
addition, vehicles may be in LoS or NLoS, with transitions from one condition to the other. This is similar to the UE walks defined in Section 5.1.1.

The channel realisations are transformed into a 2-dimensional beam gain map relating each combination of gNB and UE beam. In this case, beam gain matrices are obtained for each user and episode. As a result, each user is represented by 50 beam gain matrices describing the channel evolution of that user during the 5 seconds long simulation. And for these channel realisations, the temporal evolution of each user with beam tracking Alternative B are obtained.

In this example, the MLP network takes two features: the beam pair id, which provides a unique identifier for the combination of gNB and UE beam ids, and the beam gain experienced in the corresponding beam pair combination. Both features were normalised using the max-min normalisation. The MLP model makes predictions on the most recent $N=10$. Therefore, the input data is a matrix with 20 rows.

Three different MLP models configurations were tested.

1. A classical MLP with a single hidden layer of 1000 neurons.

2. An MLP with a hidden layer with 2000 neurons and a second hidden layer with 1000 neurons.

3. An MLP with a hidden layer with 3000 neurons and a second hidden layer with 2000 neurons, and a third hidden layer with 1000 neurons.

The three MLP configurations were trained to obtain an objective RMSE of $10^{-4}$ before completing 10,000 iterations or epochs.

Once the networks were trained, the models provided diverse prediction accuracy on new data (test set) as illustrated in Figure 6.19 for different number of neurons. MLP Network 1 offered a prediction error of $80 \%$; MLP Network 2 obtained a prediction error of 30\%; and MLP Network 3 obtained a prediction error of $9 \%$. The latest two networks outperform the accuracy achieved in the reference work in [71] with exactly the same collection of data, just by treating the input data differently.

The number of neurons has a significant impact in the training time. Network 2 took 3 hours to converge and Network 3 took approximately 1 day and 3 hours to converge. On the other hand, Network 1 did not reach the minimum RMSE threshold of $10^{-4}$ defined in the training stage. So, in order to make good predictions, the number of neurons must be sufficiently large to securely model a problem as complex as beam tracking. 


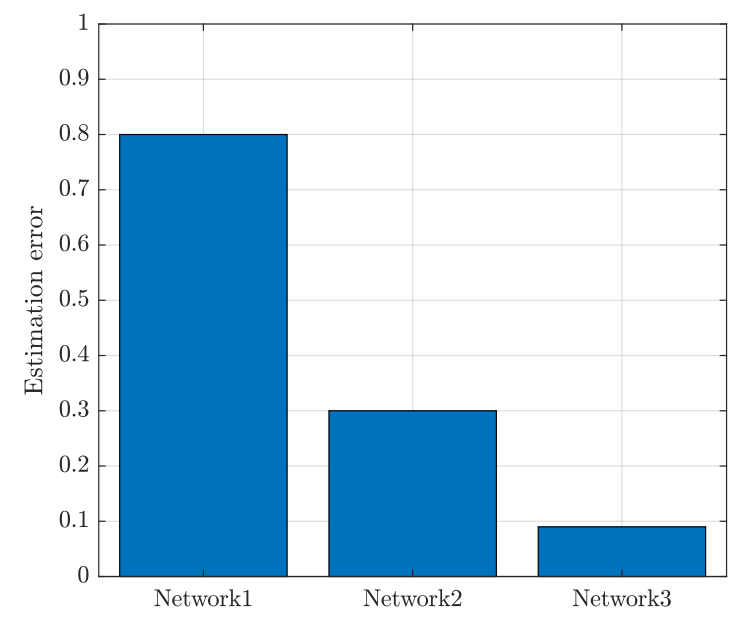

Figure 6.19: Estimation error of the test set for the three networks considered for training the MLP with the external database.

\section{Predicting beams in UMi}

In this second exercise, the ML model makes predictions on the UMi scenario. For this aim, an exhaustive collection of ray tracing measurements on the synthetic scenario was performed, with 146, 421 measurements points with a spatial resolution of 0.1 metre. This is the minimum measurement separation supported in Unity.

Following the guidelines for formatting the input data defined in Section $6.2 .3,444,396$ samples were obtained. Note that these realisations were obtained with the beam tracking Alternative B with a CSI-RS period of $10 \mathrm{~ms}$. The network takes the same input features as the previous example in Section 6.2.3, and also incorporates the user location at ground level in Cartesian coordinates. The location coordinates are also normalised with the max-min normalisation.

Since the number of samples is large, the network was initially trained and tested with a hundredth of the whole collection of samples. As a result, 4, 444 samples were randomly selected and, again, these samples were randomly split in train, validation and test sets. The network was trained with different number of neurons with a minimum RMSE threshold of $10^{-2}$. Figure 6.20 illustrates the prediction error on the test set for a MLP model with one hidden layer and 


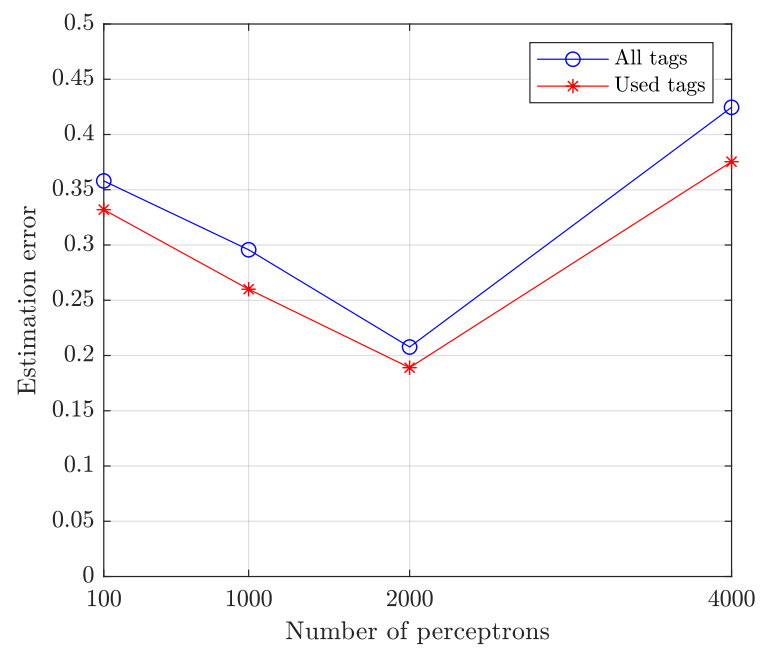

Figure 6.20: Estimation error of the test set for different number of neurons.

different number of neurons. The blue curve corresponds to the case of having 1024 outputs, one for each beam combination. The red curve corresponds to the case of having as many outputs as optimal beam combinations found in the UMi area of interest. In this case, only 124 beam pairs appeared at least once, which represents a $12.11 \%$ of all the possible beam pairs. As it can be seen in Figure 6.20, reducing the number of output helps reducing the prediction error on new data samples. By observing the estimation error for an increasing number of neurons, it can be seen that the minimum error is obtained with 2,000 neurons, but the error doubles for the double number of errors. This indicates that the ML model is overfitted to the training data: while the error on the training set is low enough, about $10^{-2}$, the estimation error on new data samples is elevated. In this training case, the fundamental reason for overfitting is the low number of samples.

Using all the samples available contributes to a better training of the network. Figure 6.21 illustrates the prediction error on the test set obtained from training an MLP model with 1,000 neurons arranged in one hidden layer and 124 beam pairs. The training exercise was performed with the whole set of samples and with a hundredth of the samples. The estimation error on the test set is $12.11 \%$ if all the samples are taken, and the error increases to a $26.00 \%$ with the hundredth of the samples. The improvement in the estimations is obtained at the expense of longer training times: training with all the samples took 32 


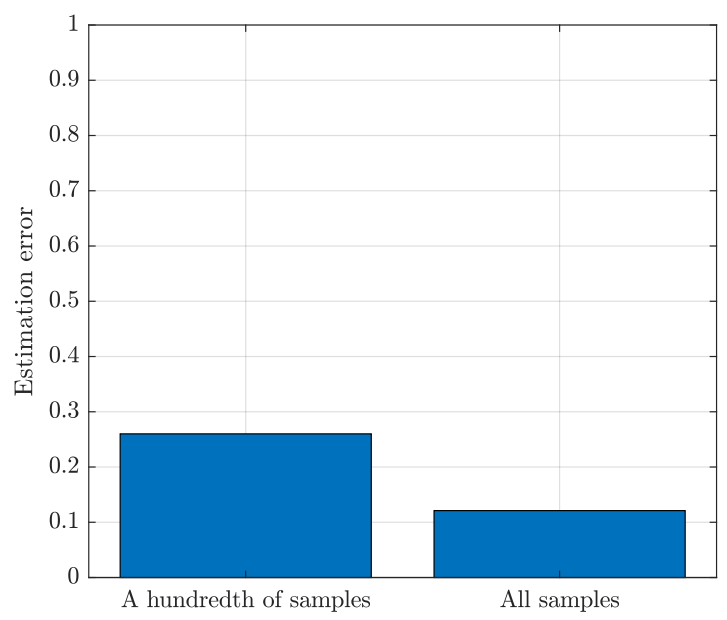

Figure 6.21: Estimation error of the test set obtained in a classic MLP with one hidden layer and 1,000 neurons for different number of samples.

hours to meet the RMSE requirement, whereas the performance objective was achieved in barely 6 minutes with a hundredth of the data samples.

It is worth highlighting that the estimation error probabilities obtained in the UMi and in the external database are similar. There is an important difference between the two networks in the number of neurons, which is 6 times smaller in the UMi training. Achieving similar performance with less neurons is due to the addition of the user location feature in the UMi case. Position information is determinant for beam tracking since the channel response at a given point depends on the geometry of the scenario.

\subsubsection{Conclusions on machine learning applications for beam tracking}

This section of the Thesis leads the way for a fruitful research activity in the ambit of ML models for beam tracking in mmWave with standardised 5G RS. This initial study has been demonstrated that ML applications for beam tracking are feasible and a promising solution to provide good beam tracking performance in different mobility situations.

The MLP model devised in this section outperforms the ML models evaluated by Klautau in [71] with exactly the same data collection, with a estimation 
error of $9 \%$ on the test set for a MLP network with 6,000 neurons. In addition, the MLP model is more realistic and easier to adapt to real $5 \mathrm{G}$ networks than the models proposed by Klautau, since the MLP model does not require assuming the knowledge of the precise location and dimensions of the blockers. The MLP model only needs the time evolution of the reported pair of beams and the beam gain. This information can be easily made available at the gNB.

In a second experiment, the ability of the MLP model in making beam predictions in the UMi scenario has been tested. Significant findings have been obtained from this exercise. For instance, the number of samples used to train the network must be sufficiently large to avoid unwanted effects such as overfitting the model to the training data. Adding more features, like the user location, can also contribute to the model prediction accuracy.

This initial results obtained with the MLP model are promising. More complex ML models may provide better prediction accuracy and possibilities should be explored. But the fundamental limitation for ML models for beam tracking is the lack of real measurements in the mmWave band. 


\section{Chapter 7}

\section{Conclusions and Future Research}

\subsection{Concluding remarks}

This Thesis has investigated the problem of beam tracking in Fifth Generation (5G) New Radio (NR) network deployments operating in the Millimeter Wave (mmWave) band. In the first place, the focus of the Thesis was on the identification of the technologies in Third Generation Partnership Project (3GPP) Release 15 of NR which enable the management of beams. In this context, this Thesis proposes implementing beam tracking strategies based on measurements of standard downlink Reference Signal (RS): Synchronization Signal (SS)/Physical Broadcast Channel (PBCH)-Block (SSB) and Channel State Information Reference Signals (CSI-RS).

Transmissions of both SSB and CSI-RS are directional, which helps detecting the promising communication directions mapped to beams. This Thesis assumes that the detection of the promising beams is done in a close-loop Channel State Information (CSI) mechanism, in which the RS are transmitted in several beams, one at a time. Those beams are extracted from a predefined codebook matrix. In alignment with $3 \mathrm{GPP}$ NR, the interest was on supporting Three Dimension (3D) beamforming in order to provide large beam gains not only in the azimuth domain but also in elevation domain.

Within this framework, this Thesis has introduced and evaluated the performance of a number of different mmWave beam tracking strategies for $5 \mathrm{G}$ networks in realistic urban scenarios under different mobility patterns. More precisely, the beam tracking techniques have been evaluated in Urban Macro- 


\section{CHAPTER 7. CONCLUSIONS AND FUTURE RESEARCH}

cell (UMa) and Urban Micro-cell (UMi) deployments. The evaluation focused on point-to-point communications, consisting on one Base Station (BS) and one User Equipment (UE), and both using analog beamforming architectures.

SSB and CSI-RS are flexible enough to provide close-to-optimal beam alignment probabilities and theoretical maximum throughput under specific mobility situations and scenarios. If Line of Sight (LoS) condition is guaranteed, then beam sweeping SSB in transmission and reception provides excellent beam tracking performance and good data rates at pedestrian speeds. Eventually, the SS burst period can be extended, resulting in a $3.87 \%$ increase of the instant data rate in the LoS zone. This is not the case of Non Line of Sight (NLoS) situations, where the time delay required to find the optimal pair of beams with beam sweeping may ruin the Signal to Interference plus Noise Ratio (SINR) and user throughput. Furthermore, in vehicular mobility patterns, the beam tracking ability is compromised if the SS burst period is set to the largest value of $160 \mathrm{~ms}$. In light of these observations, it can be said that SSB beam sweeping is a good beam tracking strategy if the mobility pattern is simple and the channel condition is favourable. In any other scenario, CSI-RS measurements are necessary to improve the performance of beam tracking. Given that the SS burst period has an impact on the initial access latency, the future $5 \mathrm{G}$ network deployments operating in the mmWave band could dynamically adjust the SS burst period to find a suitable trade-off between access latency and maximum throughput (the network should increase the SS burst period to maximise the system throughput, and it should decrease SS burst the period to reduce latency in the Random Access Channel (RACH) procedure).

Maintaining a good list of candidate pairs of beams is capital to guarantee good beam alignment. Otherwise, gaining knowledge of the channel state of all the possible beam combinations may lead to very long beam misalignment periods which reduces the SINR and, hence, limits the instant data rate experienced by the served user. Furthermore, the NR standard limits the number of NR base station (gNB) beams to monitor with CSI-RS to 8 .

The first set of beam tracking strategies presented in this Thesis complements the information obtained from the SSB periodic transmissions with additional beam measurements of CSI-RS resources. The challenge is determining which beams are worth monitoring in order to find a best alternative to the pair of beams currently in use. The first approach suggested monitoring the beams which are immediately neighbouring the active beams. In the second approach, with the aim of detecting promising directions which are distant to the current pair of beams, additional azimuth beams uniformly-separated are considered in the list of beam pairs to monitor. In general terms, both beam tracking strategies with CSI-RS measurements provided good tracking figures and data rates in LoS and, more importantly, in NLoS conditions. The use 
of CSI-RS allows relaxing the signalling overhead due to SSB transmission by enlarging the SS burst period without severely reducing the beam tracking ability. Moreover, since CSI-RS resources has a very small contribution to the signalling overhead, with a maximum percentage of $0.49 \%$ of the total resources for the Alternative B with a CSI-RS period of $10 \mathrm{~ms}$, the user throughput is not penalised by the use of CSI-RS resources.

At pedestrian speed, there is little difference between beam tracking Alternative A or Alternative B. In vehicular scenarios, Alternative B exhibits extra beam tracking resilience compared to Alternative A, especially in NLoS situations. The beam tracking must seek to optimise the beam alignment, since the better the beam alignment, the better the throughput can be. In the vehicular case, the selection of the CSI-RS period is also important. By shorting the CSI-RS period, the beam update delay is decreased. The shortest CSI-RS period of $10 \mathrm{~ms}$ has reported the best beam tracking performance and throughput in both scenarios. In conclusion, Alternative B with 10 ms CSI-RS period could be used as a one-fits-all beam tracking solution for a $5 \mathrm{G}$ network operating in the mmWave band. A future evolution of the beam management could dynamically switch strategies between one or another based on the visibility condition or other kind of considerations. But this sort of information sometimes cannot be extracted from beam measurements.

The key for an efficient beam management implementation relies on the use of the time/frequency resources. The beam management procedure must collect precise information about the channel state without wasting resources with unnecessary beam measurements. In addition to beam measurements on downlink RS, other information sources might be used for beam tracking. The use of context-aware additional information sources, like the inverse fingerprint database or the use of machine learning models to make predictions on the optimal beam pair given the UE recent history, has been proved to work.

The use of contextual information sources like the inverse fingerprinting used in this Thesis certainly helps to maintain really high data rate values and beam alignment probabilities as long as the beam information stored in the database is reliable. It has been proved that, under ideal assumptions, the inverse fingerprinting beam tracking performs as good as the beam tracking alternatives based on monitoring near-to-active beams with CSI-RS resources. Nevertheless, it has been shown that the inverse fingerprinting performance degrades under more realistic assumptions, showing a high sensitivity to noisy orientation and location measurements. Fortunately, the orientation errors in low-cost devices are within $3^{\circ}$, which prevents from suffering large beam deviations, especially in elevation. Location estimation errors are also an important source of uncertainty. It has been shown that location errors of 2 metres degrades the beam tracking ability of the inverse fingerprinting. Such level of 
precision is, in practice, very challenging in dense urban scenarios. In fact, the location error requirement that has been found in technical documents sets a less demanding maximum error of 7.8 metres. It is this high-precision location requirement which makes inverse fingerprinting beam tracking specially challenging. Still, in a multi-user scenario, the overhead due to CSI-RS might be an important issue. In this case, the inverse fingerpinting approach can help to reduce the signalling burden. In a future extension of the Thesis, it would be interesting to assess what reduces the experienced throughput more severely, the location error or the increased signalling overhead due to a high number of users connected to a serving BS.

Machine Learning (ML) models for beam management are also an interesting research direction which is worth exploring. The shallow neural network used in this Thesis makes reasonably accurate predictions on the optimal pair of beams with only providing a few number of the most recent beams used by the UE and their associated beam gain. The Multi-Layer Perceptron (MLP) model used provided a success probability of about $91 \%$ for the reference data set, which sets a promising starting point.

This Thesis has proved that high capacity links can be obtained with the wide beams used for beam sweeping. This will reduce the complexity of future $5 \mathrm{G}$ Standalone (SA) network deployments and reduce the sensibility to beam misalignments. The requirement of having narrow beams in mmWave is not important in the single-user scenarios evaluated in this Thesis, and the extra beam gain of narrow beams might be of interest in NLoS situations with many reflections so the path loss needs to be compensated.

The rotation of the user device clearly impacts the beam tracking performance, especially on the UE side. For instance, beam tracking solutions must consider rotation information obtained from gyroscopes, which are available in many smartphones and devices, to compensate or to adapt the suggested pair of beams to the rotation applied to the device.

\subsection{Future research directions}

The work done in this Thesis should extend the beam tracking analysis to multi-user scenarios, where users may interfere severely. In such scenarios, interference reduction and mitigation techniques play a significant role. A straight-forward interference reduction technique related to beam management is the use of narrower beams at both transmitter and receiver. An immediate effect of reducing the beam area is that more beams will be necessary to beam sweep the same area. This may induce delays in the RACH procedure since the necessary number of transmit SSBs could be increased and, eventually, the 
number of necessary SS burst cycles may be larger. Hierarchical codebook design $[52,121,122]$ can achieve interference reduction without enlarging the RACH procedure. With this kind of codebooks, SSB may be transmitted with the first level of wide beams, and the second level of narrow beams shall be used for fine beam tune and CSI-RS beam measurements. In such case, the beam management mechanism must relate each wide SSB beams to narrower with the decomposed narrower beams for CSI-RS. One critical question regarding beam tracking with multi-level hierarchical codebooks is how many narrow beam pairs are required to monitor with CSI-RS resources. This number will pose a limit on the number of users connected simultaneously before the signalling overhead due to CSI-RS resources reduces the system throughput.

The use of non-static beamforming vectors should be considered too. For instance, in an event of a reduction of the beam tracking performance due to the mobility pattern of the users, or due to a rapidly changing environment, the network may react and change to a codebook with wider beams, and return to a more directional codebook once the normal situation is recovered. In alignment with this, the use of fully-digital beamforming architectures will be normalised in the later $5 \mathrm{G}$ deployments and can reshape the beam to point the target user and, at the same time, reducing the interference induced to other users in the vicinity [123].

In this thesis, imperfections in digital or analog transmission systems with multiple antennas have not been taken into account, apart from the feedback delay. The literature offers us little analysis on other sources of imperfection, although in [124], for example, it is shown that certain problems of implementation of the phase shifters can have a serious effect on the accuracy of the codewords. This issue should also be investigated in future work.

Another system model extension to be considered is deploying several mmWave BSs. The NR standard contemplates that gNBs exchange beam information among them. This information shall be used by each beam management entity to detect situations where a UE may get a better channel quality and service with another BS and should be handed over.

After the completion of above-mentioned research works, the following step should be testing the beam tracking strategies in real deployments. This exercise would serve to validate the simulation results obtained in this Thesis. Repeating this exercise in different scenarios can contribute to obtain a large collection of real measurements, which can be used to conceive more accurate ML models. Real data scarcity is the main limiting factor of ML models for 5G, not only in the ambit of the beam tracking topic discussed in this Thesis, but also in other Physical (PHY) parameters, efficient resource allocation, interference avoidance, and Radio Access Technology (RAT) automatic parameter optimisation. In the meantime, the currently-active open-access channel mea- 
surement collection initiatives like NIST's will keep growing due to the number of $5 \mathrm{G}$ key players involved in the initiative. This growth will determine the time readiness of the ML models.

As data sources become more numerous and complete, simple ML models may become inefficient. In such situation, deep learning Artificial Intelligence (AI) models are indicated to manage enormous volumes of data. Convolutional Neural Network (NN) (CNN) and Recurrent NN (RNN) are experiencing a great success in a wide range of applications. The input data must be formatted specifically to the selected type of deep NN. As mmWave $5 \mathrm{G}$ deployments gain presence in typical urban scenarios, more features can be added to an eventual ML model for beam tracking. The device orientation is determinant in the optimal beam selection. And this orientation should be taken into account if UE beams are to be predicted. In a future world where everything is connected, the network may learn the traffic patterns of users at the current time of the day and make beam suggestions based on their most likely direction of movement. Other features worth exploring are user speed, network parameters or Key Performance Indicator (KPI)s such as Radio Link Failure (RLF), list of detected intra-RAT and inter-RAT cells, etc.

Nowadays, operators are starting deploying their $5 \mathrm{G}$ networks operating in Frequency Range (FR) 1 (FR1). However, real 5G networks operating in mmWave would arrive at the end of the $5 \mathrm{G}$ cycle, eventually at the same time the first Sixth Generation (6G) networks start to be deployed. Network densification will play a significant role in the success of wireless networks operating in the mmWave band. Densification can certainly increase the LoS probability. In a blockage event, it would be desirable to hand the connection over a different mmWave BS, possibly in LoS, in order to maintain the current Quality of Service (QoS).

The lessons learned in the study of beam tracking strategies for mmWave networks can be extended beyond the $5 \mathrm{G}$ frequency limit of $100 \mathrm{GHz}$. The Terahertz frequency band is in consideration for the future 6G networks [125]. But before arriving there, real mmWave wireless networks must prove to be resilient in mobility environments. 


\section{Appendix A}

\section{Visualisation platform}

\section{A.1 Introduction}

The upcoming revolution in the wireless industry, the so-called Fifth Generation $(5 \mathrm{G})$, will extend cellular communications to a wide range of novel applications and use cases, involving different players: vertical industries, new stakeholders, standardisation bodies, policy-makers and regulators. As complex as the $5 \mathrm{G}$ technology will be, it will be of paramount relevance to explain the solutions, frameworks and benefits that these new technologies will bring to society.

An extremely useful way to do this is through the visualisation of the technology operation and its performance. So far, the visualisation of wireless communications concepts has been limited to 1) the provision of static contents -such as figures, pictures or diagrams- in presentations or scientific papers; 2) videos with pre-recorded information in a highly-controlled environment; or 3) simulation tools with archaic Graphical User Interface (UI) (GUI) that display the simulation results after tuning some controlled parameters [126]. These traditional visualisation options have important limitations, such as the lack of interactivity or the absence of user immersion. These drawbacks, that prevent them from being effective to today's over-stimulated audiences accustomed to Virtual Reality (VR) or Augmented Reality (AR) applications.

Immersive experiences have demonstrated to augment the ability to understand multifaceted datasets [127]. Accordingly, the 5G-related METIS-II project [30] identified the need to work on a new visualisation paradigm, very realistic and more aware of the user experience, and relying on a good story to tell [128]. The selected paradigm was the use of a serious-game in which visualisation is in fact a video game where story, art and software, contribute 


\section{CHAPTER A. VISUALISATION PLATFORM}

to a pedagogic aim [129]. In addition, when creating visualisation content to explain ideas, the inclusion of appealing elements increases intuitiveness and memorability [130]. User interactivity is approached with gaming concepts. The introduction of elements from the gaming ambit has successfully provided large benefits for knowledge gain and retention in a wide range of ambit areas including healthcare, entertainment, military, engineering or sports [131].

In the ambit of telecommunication engineering, a group of researchers created a game to introduce students to cellular network planning in the context of reestablishing communications after a natural disaster [132]. The weak aspect of this tools is the use of statistical channel models, highly inaccurate from reality, and having the game limited to a single scenario use case. For example, METIS-II visualisation platform was limited to a single realistic scenario [109]. The tool presented in [133] provided good user interaction and game immersion but it was limited to the graphical representation of simulation data from an external source conveniently formatted to the platform.

This appendix introduces the contribution to the immersive open-source Visualisation Platform (VP) for visualising 5G network aspects developed in the ambit of METIS-II project [30]. This work resulted in a magazine paper [109], a conference paper [134] and a project deliverable [135].

\section{A.2 5G visualisation}

To create an effective visualisation platform, it is necessary to understand the science that is to be visualised [136]. If a non-expert is asked about what the $5 \mathrm{G}$ is or will be, the answer will be likely something related to the increase in browsing speed. But the $5 \mathrm{G}$ is not only that. The $5 \mathrm{G}$ paradigm is to change many things in the system, but also affects the way to communicate and who will be the agent of communication.

$5 \mathrm{G}$ visualisation emerges as a unique opportunity to make pedagogy with this shift on our understanding of mobile communications and must result in a tool that allows its users to focus on different levels of conceptual complexity. In the METIS-II project's structure, these concepts are grouped into Key Innovation Pillars. These pillars are not only considered in the use case scenario origins, but also sets the foundations of visual and data driven elements and examples on which the future investigation into the areas of interest can be initiated.

To increase the educational capability of the visualisation platform, METISII decided to present the $5 \mathrm{G}$ concepts in the visualisation using the storytelling approach [137], organising the visualisation into parts with a rational continuous flow that would start with a general view of $5 \mathrm{G}$ and would finish with 
more detailed concepts. Nevertheless, as the story evolved the user could have the possibility to choose its own end by stopping in specific parts of it where, eventually, the user could build its own story from there. The visualisation platform is able to work on different levels of abstraction, as it can use schematic views of concepts for high levels of abstraction and realistic Three Dimension (3D) options for low levels of abstraction.

\section{A.3 Open source graphical environment}

METIS-II visualisation platform should be open-source with the objective of convincing $5 \mathrm{G}$ researchers and companies to use the tool for presenting their results and perform visual demonstrations, and to foster collaboration between different projects. METIS-II has released its visualisation platform and manual in its web page [30], and has based its development on the Unity game engine that provides many open source materials [135].

However, to achieve this wide use objective, allowing the developers to create the visualisation examples the way they want it is also necessary i) to have a highly customisable platform, and ii) to have a simple and well-defined methodology to transform scientific data into visual representations.

In the beginning of the project it was suggested that the scientific data, including the raw data coming from computer simulations, could be directly passed to the visualisation platform, which would do the data processing to obtain the visualisation inputs. In fact, it was planned to build a common data base where the simulator would store their results and the visualisation platform would read them. It was also planned to define the interface between this general-purpose data base and the visualisation platform. This option was later withdrawn before Mobile World Congress (MWC) 16 (MWC16), after realising the diversity of the concepts to be visualised, as explained in the previous section, and the enormous processing power needed in the machines to carry pure visualisation tasks and data processing. Instead, it was decided that the visualisation platform would be just that, a tool for visualising concepts and responsible for turning data into visual entities, but also performing other actions such as data management. Therefore, simulation results should be processed outside the visualisation tool and the result of such processing should clearly indicate to the visualisation tool what to visualise and how. This approach requires, then, to provide the scientists a plurality of visualisation options in the platform, and a clear way of indicating the tool what to visualise.

To provide the plurality of visualisation options, the platform provides a library with numerous graphical assets and options that eases the setup of any potential concept to be visualised. 


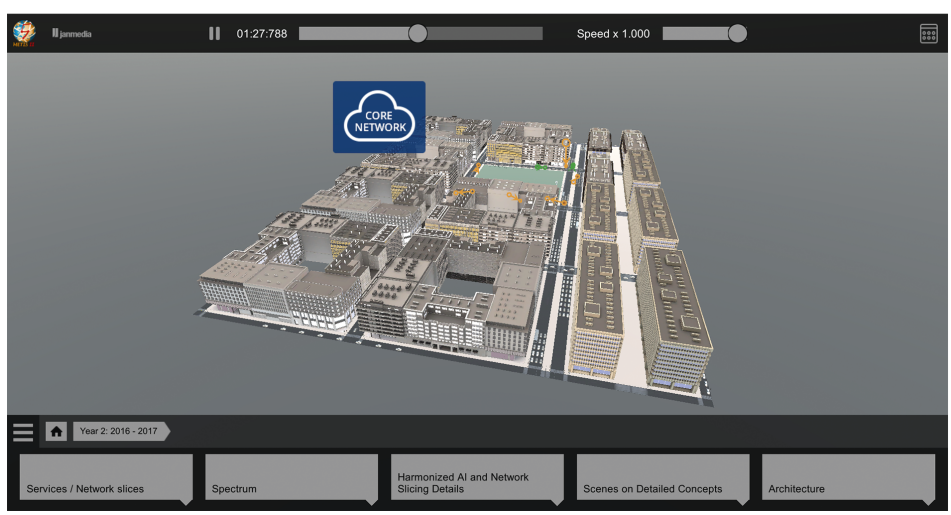

Figure A.1: Bird's eye view of Madrid grid scenario.

Visualisation objects are Two Dimension (2D) or 3D. Perhaps, the most important 3D object is the scenario. In the context of the METIS-II project, a realistic urban scenario named Madrid grid, described in [138], was the elected scenario to visualise the key $5 \mathrm{G}$ concepts on. This scenario resembles a typical European city centre whose main elements are: multiple blocks of multi-storied buildings, a park, medium-width streets, a wide avenue, and a pedestrian commercial street. One interesting characteristic of the implementation of the Madrid grid scenario is that it allows applying different skins to buildings. A bird's-eye view of the Madrid grid 3D scenario implemented in the METIS-II visualisation platform is shown in Figure A.1.

The visualisation platform was designed to allow the loading of any $3 \mathrm{D}$ scenario compatible with Autodesk FBX format. The key aspect of the FBX format is its interoperability with any digital content creation software, so developers are not constrained to a single creation tool, instead, they may use their favourite one, including open source solutions like Blender.

The visualisation platform also provides a set of predefined $3 \mathrm{D}$ models, also known as components, for urban areas such as pedestrians, cars, buses, traffic lights, lampposts and cellular antennas. The collection of components is completed with physical and logical networking elements models of routers, antenna beams, coverage areas or spheres. The editor or the developer of the visualisation platform may of course configure the size, colour, location and orientation of the objects through the whole visualisation. An example of the scenario populated with 2D and 3D objects is shown in Figure A.2.

The platform provides also $2 \mathrm{D}$ objects to be visualised. However, $2 \mathrm{D}$ objects are treated differently to $3 \mathrm{D}$ objects in the sense that $2 \mathrm{D}$ objects do not occupy 


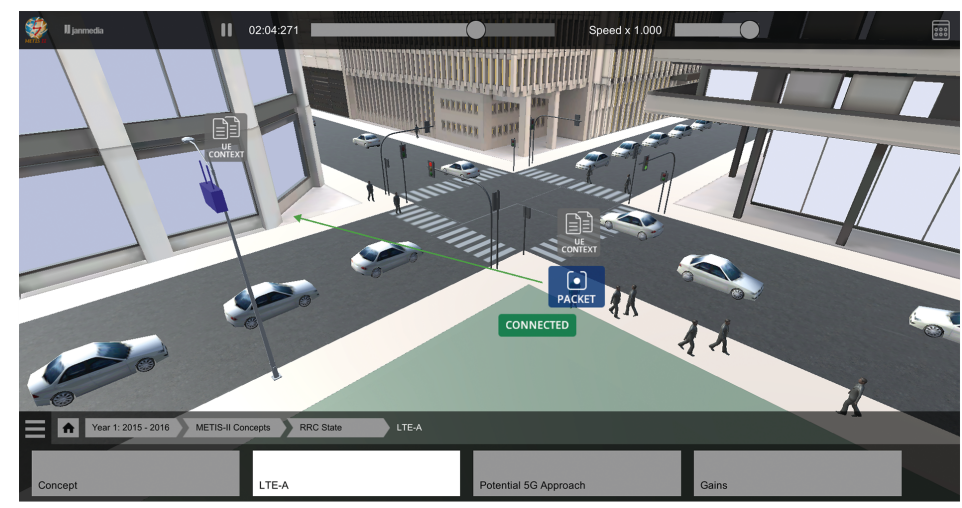

Figure A.2: An example of the Madrid grid scenario with different 2D and 3D visualisation material.

an area in the virtual space; this is consistent with how Unity manipulates those objects. 2D objects are displayed over the GUI panel. The variety of $2 \mathrm{D}$ objects included in the visualisation platform goes from simple forms like lines and circles to sophisticated objects such text boxes, buttons, bar diagrams, graphs and widgets. The visualisation platform also supports the use of external images to form small icons or larger pictures like, for example, a slide of a presentation.

The visualisation platform can be understood as a window to a $3 \mathrm{D}$ world where different views show different scenes to illustrate the main $5 \mathrm{G}$ concepts. With that aim, each view contains a specific set of visualisation objects and focuses on a specific area of the Madrid grid.

For each view there is an associated button in the menu bar in the lower part of the GUI. As it can be seen in Figure A.2, each button has a label that unequivocally identifies the view, and the user of the visualisation platform can easily jump from one view to the next one by interacting with the displayed buttons. Views can be related one to the next one as chapters in a book. However, it is possible to define views inside a view with the intention, for instance, of modifying some of the visualisation content of the base view by selecting the different options. To help the user in the navigation along multilevel views, the GUI displays buttons to the views the user has visited. There is also a home button that takes the user to the main view.

In order to customise the views shown, the platform provides an Extensible Markup Language (XML) file whose structure corresponds to the view structure finally shown in the computer program. 


\section{CHAPTER A. VISUALISATION PLATFORM}

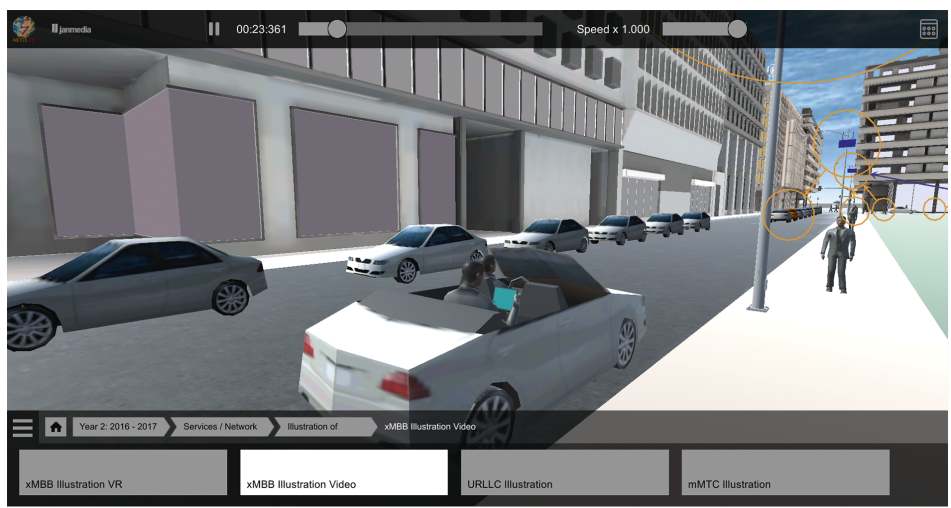

Figure A.3: An example of first person view in the visualisation platform.

The main view is the very first view the user sees when the visualisation platform starts. Its corresponding XML section includes the definition of the visualisation objects that are common to all the views, including the scenario and, optionally, other visualisation materials.

In general, a view section of the XML defines the list of visualisation objects to be include but it also contains the definition of the properties in time of the associated visualisation objects. For instance, traffic lights switch their light phases following a periodic pattern that is fully customisable at the main configuration file.

Although the XML sets the initial configuration of some elements, the user may interact with the tool and change them in real time. For example, the camera setup is fully customisable at each view. By adjusting the position and orientation of the camera, the editor can control the initial perspective of the scenario that is going to be displayed. The camera can focus, for example, in one corner of the scenario as in Figure A.2. In real time, the user can also zoom in and out and move around the scenario with a mouse and a keyboard. First person views such as the one shown in Figure A.3 can be provided, which contributes to an enhanced immersion of the user. Another interesting aspect of the camera is the possibility to set a visualisation point of interest. If the platform user selects a visualisation object, the camera will focus on that object. This means that in case of selecting a moving component like a vehicle, the camera will smoothly change to a third person view and will follow the object from its rear side.

To facilitate the introduction of mobile elements such as cars, the visualisation platform may read binary files, with a well-defined structure that indicate 


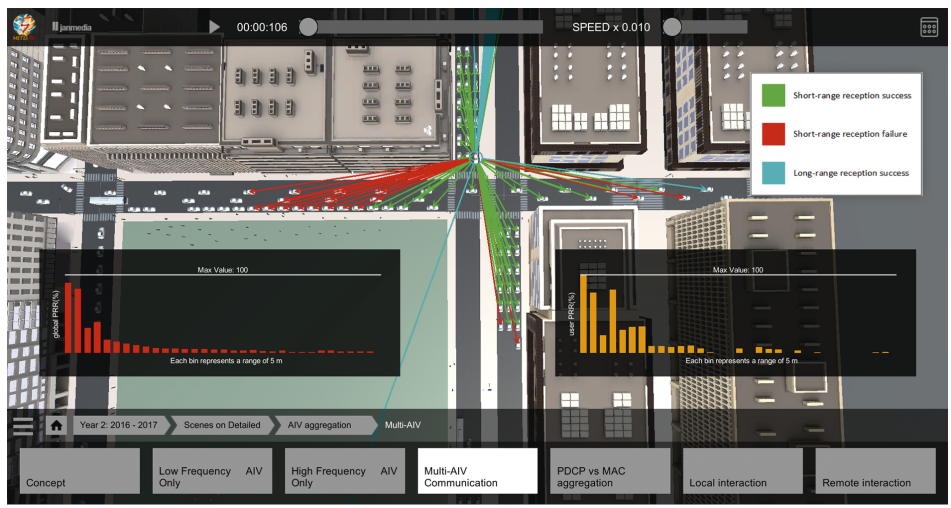

Figure A.4: Visualisation example of communication between cars.

the position and orientation of each mobile element in periodic instances of time. For each view, a different mobility trace can be loaded thanks to the XML configuration commands.

Charts, graphs, arrows, and other graphical elements may be shown in a view to reflect the information from simulations. This rich information is also read from binary files with a structure well-defined to allow interoperability of the visualisation platform with multiple simulators.

One good example of the use of this simulation data is provided in Figure A.4, which illustrates the communication between cars in 5G. In this illustration, the transmission of information between cars is shown as arrows. Green and blue arrows mean a correct transmission of information, while red arrows denote errors in the transmission and coverage problems. That visual information is enriched with a small picture on the top right corner describing the colour code used and with two bar charts displaying two different network metrics (probability of reception of information vs distance). The location and size of the chart and its number of bars, colours, legends and texts are fully customisable and defined at the main visualisation configuration file.

The same applies to the diagram charts illustrated in the example in Figure A.5. In this case, what is illustrated is the concept of slicing where network metrics from two slices are shown. In both examples, the values represented are periodically updated according to the update information in the binary file with the simulation data.

In Figure A.6, an additional capability of the platform is shown. In this case, the buildings are made transparent to show the position of devices located indoors and the connections between those devices. 


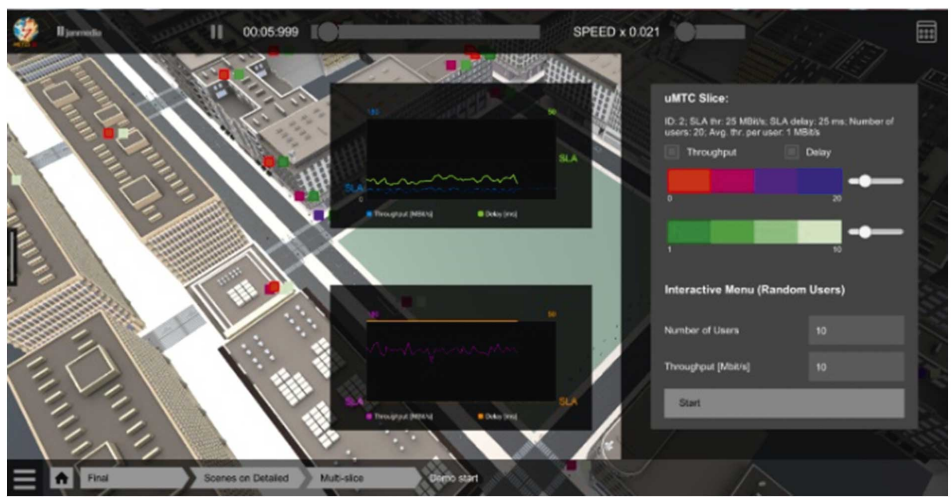

Figure A.5: Visualisation example of the slicing concept.

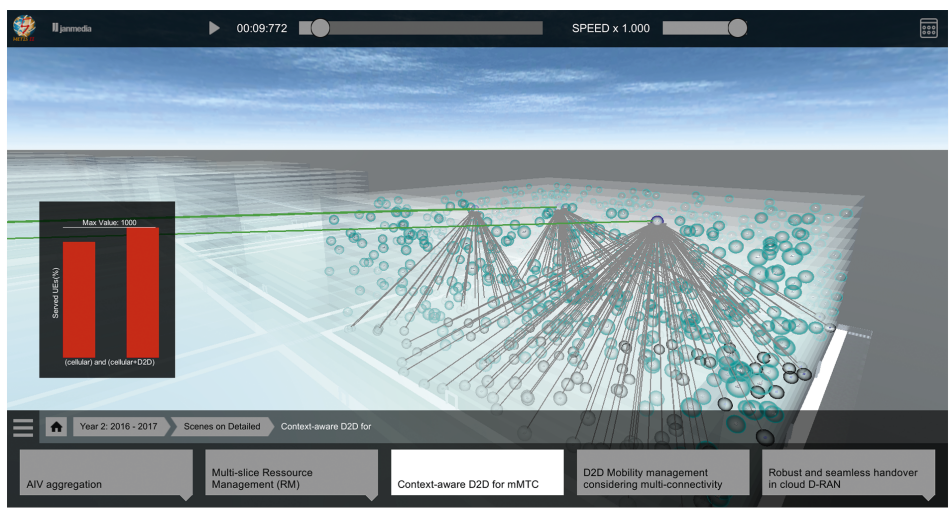

Figure A.6: Visualisation example of communication among devices. 


\section{A.4 Interaction capabilities}

\section{A.4 Interaction capabilities}

The interactive visualisation provides two main benefits [139]. In the first place, the interaction, i.e. the dialog between the user and the system, keeps the user attention, due to its active involvement in the visualisation process. And secondly, interactivity allows the user to have a customised user experience by making changes on the visualised information or the way in which information is visualised. This customisation capability was presented as the capability of the user to decide the final of the story-telling in Section A.2.

The first degree of interactivity is achieved thanks to the use of navigation bars and menus. In fact, the lower part of the visualisation screen presents a navigation area that allows the user to move from one $5 \mathrm{G}$ concept to another for example.

In some concepts, the visualisation platform may show $3 \mathrm{D}$ scenes where the visualised elements change or evolve in time, for example, cars moving along the streets, or graphical representations of performance indicators, such as the download speed achieved by a smartphone. The user can control the temporal evolution of the visualisation using controls located in the upper part of the UI. Specifically, the user can accelerate or decelerate the temporal evolution, and jump to specific time instances thanks to the use of two sliding bars. The user can also stop and resume the visualisation using the button for such purposes.

A very useful interaction capability consists on the ability of the user to change parameters of the concepts visualised. In the METIS-II platform, this can be achieved by changing from one menu to another one, when the results shown in those menus are obtained with different parameters that the user wants to compare. But, a more interesting way of doing this interaction is by using widgets, filled with fields where the user can select the values of some parameters. For example, Fig. 6 includes a widget integrated in the right side of the UI. The visualisation platform comes with a few predefined widgets for a specific use but it also offers a modular widget that allows the editor to create a target-purpose widget at the visualisation configuration file.

The inherent problem of this option is that the visualisation tool should store a huge amount of information if the set of values that the user could select is high, making impossible to let the user choose the parameters freely, not from a prefixed set.

METIS-II overcame this problem by allowing the visualisation platform to interact in real-time with external simulators. Those simulators could run in the same computer where the visualisation tool is running, or in remote servers. To enable this simulation-visualisation interworking in real time, internet sockets are used and the structure of the messages to be exchange between both pairs have been specified in the platform manual. Those messages allow the 
indication of the simulation parameters from the visualisation platform to the simulator, with certain update period allowed, and the periodic sending of graphical representation information from the simulator to the visualisation platform. This paradigm has proven to be successful and both academic partners, such as the Universitat Politècnica de València (UPV) and the University of Kaiserslautern, and industrial partners such as Deutsche Telekom, have successfully demonstrated the real-time interworking of the visualisation platform and their simulators in real time. For those interested readers, more technical details on the interworking are provided in [135] where instructions to work with a publicly available simulation server from UPV are provided.

\section{A.5 Lessons learned}

The development of the METIS-II visualisation platform was a challenging process that has provided meaningful insights on several visualisation aspects that will be useful in the future for the platform developers, and for those making a similar exercise.

The visualisation platform design was a joint effort of artists without any previous knowledge about the ICT industry and $5 \mathrm{G}$ experts. This joint work was facilitated by the original intuition of METIS-II, that in its first phase elaborated a simple story to explain the $5 \mathrm{G}$. This pedagogical work was really useful to make the artists understand the problem that they were facing. Another factor that made easy the inter-working was the specific profile of the $5 \mathrm{G}$ researchers, many of them with grades in telecommunications and familiar, although non-experts, with multimedia, games and other aspects involved in the development of the visualisation platform. In fact, some $5 \mathrm{G}$ researchers that are more visualisation-oriented were real catalysts of the work. Therefore, the lesson is that the more familiar are artists and researchers with the work of the other, the easier and more productive will be the joint work.

In the beginning of the METIS-II project, long time was spent in discussing about the interface between simulation results and the visualisation platform, trying to define an interface able to accommodate all the possible results. However, the solution was really simple and defended by the visual-oriented $5 \mathrm{G}$ researchers: the simulation results should be processed by the researchers to tell the visualisation platform what to visualise and how. This approach, although implies some work of the researchers to process their results, simplifies the development of the platform and the introduction of new concepts, since this introduction may be done in most cases without any change in the platform software. As a conclusion, if a visualisation platform is to be used by multiple researchers with different interests, it is very useful to build the platform as 
a graphical general-purpose environment in which the researchers will be the ones elaborating their own instances of the visualisation tool.

Some METIS-II partners were initially reluctant to make the effort to produce advanced visualisations of their research. Nevertheless, most of them changed their mind when they saw the success in the firs great demonstration milestone of METIS-II, the MWC16. In this project, the visualisation focused on vehicular communications got the attention of public and politics, for example. A valuable lesson learned from this experience is that a successful visualisation story is worth more than a large explanation to the researchers.

To conclude, after MWC16, the first release of the platform was extended and matured. Part of this process consisted on elaborating a detailed manual on the use and capabilities of the platform. This work done by the artists and a first set of researchers was really useful for other partners since the time spent by them from the idea conception to the visualisation was drastically reduced, even when they required from the artists new visualisation options with the corresponding specification of the information format to feed the visualisation platform.

\section{A.6 Conclusions}

The benefit of using realistic scenarios is the possibility of displaying scenariospecific data in context-aware situations. Think of an overcrowded open area and the mobile network is under high traffic congestion. The VP could be of great value to operators to quickly identify the potential locations where to add small cells, with reduced coverage area, to offload traffic from the saturated base stations. Then engineers could adapt the narrative used in the VP to their managers to show how their solution works and its viability from an economic point of view should be studied. Typically, small cells need fiber optics connection to the operator's core network, but the infrastructure costs could soar when the number of cells becomes high. Not to mention that construction works could bring several bureaucratic troubles. A good alternative is to communicate small cells with a base station by means of high-capacity Millimeter Wave (mmWave) backhaul links. Our VP can certainly identify which locations could support such interesting links from the operator point of view. From this example, it can concluded that the VP can address different target users with the appropriate context and visualisation material. 



\section{References}

[1] ITU-R, "Framework and Overall Objectives of the Future Development of IMT for 2020 and Beyond," International Telecommunication Union (ITU), M 2083-0, Oct. 2015.

[2] 3GPP TSG RAN, "NR; Overall Description; Stage-2," 3rd Generation Partnership Project (3GPP), Technical Specification (TS) 38.300, Jun. 2018, version 15.2.0.

[3] 3GPP TSG RAN, "5G Timeline," 3rd Generation Partnership Project (3GPP), SP 150140, Mar. 2015.

[4] 3GPP TSG RAN, "Study on Channel Model for Frequency Spectrum Above $6 \mathrm{GHz}$," 3rd Generation Partnership Project (3GPP), Technical Document (TDoc) RP-160210, Mar. 2016.

[5] 3GPP TSG RAN, "Study on Channel Model for Frequency Spectrum Above $6 \mathrm{GHz}$," 3rd Generation Partnership Project (3GPP), Technical Report (TR) 38.900, Jun. 2016, version 14.0.0.

[6] 3GPP TSG RAN, "Study on Channel Model for Frequencies From 0.5 to $100 \mathrm{GHz}$," 3rd Generation Partnership Project (3GPP), Technical Report (TR) 38.901, Jun. 2017, version 15.0.0.

[7] 3GPP TSG RAN, "Study on Scenarios and Requirements for Next Generation Access Technologies," 3rd Generation Partnership Project (3GPP), Technical Document (TDoc) RP-160811, Dec. 2016.

[8] 3GPP TSG RAN, "Study on Scenarios and Requirements for Next Generation Access Technologies," 3rd Generation Partnership Project (3GPP), Technical Report (TR) 38.913, Apr. 2016, version 14.0.0.

[9] ITU-R, "Minimum Requirements Related to Technical Performance for IMT-2020 Radio Interface(s)," International Telecommunication Union (ITU), M 2410-0, Nov. 2017. 
[10] 3GPP TSG RAN, "Study on New Radio (NR) Access Technology," 3rd Generation Partnership Project (3GPP), Work Item (WI) RP-170379, Dec. 2016.

[11] 3GPP TSG RAN, "Study on New Radio (NR) Access Technology," 3rd Generation Partnership Project (3GPP), Technical Report (TR) 38.912, Mar. 2016, version 14.0.0.

[12] 3GPP TSG RAN, "Study on New Radio (NR) Access Technology," 3rd Generation Partnership Project (3GPP), Work Item (WI) RP-181726, Mar. 2018.

[13] “3GPP Web Site," https://www.3gpp.org/, accessed: 2019-09-29.

[14] 3GPP TSG RAN, "NR; User Equipment (UE) Radio Transmission and Reception; Part 1: Range 1 Standalone," 3rd Generation Partnership Project (3GPP), Technical Specification (TS) 38.101-1, Jul. 2019, version 15.6.0.

[15] 3GPP TSG RAN, "New Frequency Range for NR (24.25-29.5 GHz)," 3rd Generation Partnership Project (3GPP), TS 38.815, Jul. 2018, version 15.0.0.

[16] C. Lim, T. Yoo, B. Clerckx, B. Lee, and B. Shim, "Recent Trend of Multiuser MIMO in LTE-Advanced," IEEE Communications Magazine, vol. 51, no. 3, pp. 127-135, Mar. 2013.

[17] 3GPP TSG RAN, "Radio Frequency (RF) Requirement Background for Active Antenna System (AAS) Base Station (BS)," 3rd Generation Partnership Project (3GPP), Technical Specification (TS) 37.847, Jan. 2018, version 15.0.0.

[18] 3GPP TSG RAN, "Spatial Channel Model for Multiple Input Multiple Output (MIMO) Simulations," 3rd Generation Partnership Project (3GPP), Technical Report (TR) 25.996, Jun. 2018, version 15.0.0.

[19] 3GPP TSG RAN, "Study on 3D Channel Model for LTE," 3rd Generation Partnership Project (3GPP), TR 36.873, Jan. 2018.

[20] ITU-R, "Guidelines for Evaluation of Radio Interface Technologies for IMT-Advanced," International Telecommunication Union (ITU), M 2135-1, Dec. 2009. 
[21] H. Zhao, R. Mayzus, S. Sun, M. Samimi, J. K. Schulz, Y. Azar, K. Wang, G. N. Wong, F. Gutierrez, and T. S. Rappaport, " $28 \mathrm{GHz}$ Millimeter Wave Cellular Communication Measurements for Reflection and Penetration Loss in and Around Buildings in New York City," in 2013 IEEE International Conference on Communications (ICC), Jun. 2013, pp. 51635167.

[22] Z. Pi and F. Khan, "An Introduction to Millimeter-Wave Mobile Broadband Systems," IEEE Communications Magazine, vol. 49, no. 6, pp. 101-107, Jun. 2011.

[23] T. S. Rappaport, S. Sun, R. Mayzus, H. Zhao, Y. Azar, K. Wang, G. N. Wong, J. K. Schulz, M. Samimi, and F. Gutierrez, "Millimeter Wave Mobile Communications for 5G Cellular: It Will Work!" IEEE Access, vol. 1, pp. 335-349, May 2013.

[24] S. Rangan, T. S. Rappaport, and E. Erkip, "Millimeter-Wave Cellular Wireless Networks: Potentials and Challenges," Proceedings of the IEEE, vol. 102, no. 3, pp. 366-385, Mar. 2014.

[25] G. R. Maccartney, T. S. Rappaport, S. Sun, and S. Deng, "Indoor Office Wideband Millimeter-Wave Propagation Measurements and Channel Models at 28 and $73 \mathrm{GHz}$ for Ultra-Dense 5G Wireless Networks," IEEE Access, vol. 3, pp. 2388-2424, Oct. 2015.

[26] S. Hur, Y. J. Cho, J. Lee, N.-G. Kang, J. Park, and H. Benn, "Synchronous Channel Sounder Using Horn Antenna and Indoor Measurements on $28 \mathrm{GHz}$," in 2014 IEEE International Black Sea Conference on Communications and Networking (BlackSeaCom), May 2014, pp. 83-87.

[27] S. Hur, Y. J. Cho, T. Kim, J. Park, A. F. Molisch, K. Haneda, and M. Peter, "Wideband Spatial Channel Model in an Urban Cellular Environments at $28 \mathrm{GHz}$," in 2015 9th European Conference on Antennas and Propagation (EuCAP), May 2015, pp. 1-5.

[28] S. Salous, S. M. Feeney, X. Raimundo, and A. A. Cheema, "Wideband MIMO Channel Sounder for Radio Measurements in the $60 \mathrm{GHz}$ Band," IEEE Transactions on Wireless Communications, vol. 15, no. 4, pp. 2825-2832, Apr. 2016.

[29] S. Hafner, D. A. Dupleich, R. Muller, J. Luo, E. Schulz, C. Schneider, R. S. Thoma, X. Lu, and T. Wang, "Characterisation of Channel Measurements at 70GHz in Indoor Femtocells," in 2015 IEEE 81st Vehicular Technology Conference (VTC Spring), May 2015, pp. 1-5. 
[30] METIS, "METIS channel models," Deliverable D1.4, Jul. 2015, https: //www.metis2020.com/documents/deliverables/.

[31] Winner-II, "Channel Models," IST-4-027756 WINNER II project, Deriverable D.1.2, 2008, http://www.ist-winner.org/deliverables.html.

[32] Winner+, "Final Channel Models," CELTIC CP5-026 WINNER+ project, Deriverable D.5.3, 2010, http://projects.celtic-initiative.org/ winner+/deliverables_winnerplus.html.

[33] R. Verdone and A. Zanella, Pervasive Mobile and Ambient Wireless Communications. COST Action 2100. Springer, 2012.

[34] A. Maltsev, V. Erceg, and E. Perahia, "Channel Models for $60 \mathrm{GHz}$ WLAN Systems," Institute of Electrical and Electronics Engineers (IEEE), Document IEEE 802.11-09/0334r8, 2010.

[35] Z. Zhong, X. Yin, X. Li, and X. Li, "Extension of ITU IMT-Advanced Channel Models for Elevation Domains and Line-of-Sight Scenarios," in 2013 IEEE 78th Vehicular Technology Conference (VTC Fall), Sep. 2013, pp. $1-5$.

[36] J. F. Monserrat, R. Fraile, and L. Rubio, "Application of Alternating Projection Method to Ensure Feasibility of Shadowing Cross-Correlation Models," Electronics Letters, vol. 43, no. 13, pp. 724-725, Jun. 2007.

[37] R. E. Hattachi and J. Erfanian, "5G Channel Model for bands up to 100 GHz," Next Generation Mobile Networks (NGMN), White Paper, Dec. 2015, http://www.5gworkshops.com/5GCM.html.

[38] G. R. Maccartney, M. K. Samimi, and T. S. Rappaport, "Omnidirectional Path Loss Models in New York City at $28 \mathrm{GHz}$ and $73 \mathrm{GHz}$," in IEEE International Symposium on Personal, Indoor and Mobile Radio Communications, PIMRC, Sep. 2015, pp. 227-231.

[39] M. Hata, "Empirical Formula for Propagation Loss in Land Mobile Radio Services," IEEE Transactions on Vehicular Technology, vol. 29, no. 3, pp. 317-325, Aug. 1980.

[40] T. A. Thomas, H. C. Nguyen, G. R. MacCartney, and T. S. Rappaport, "3D mmWave Channel Model Proposal," in 2014 IEEE 80th Vehicular Technology Conference (VTC2014-Fall). IEEE, Sep. 2014, pp. 1-6. 
[41] S. Sun, T. S. Rappaport, T. A. Thomas, and A. Ghosh, "A Preliminary 3D mmWave Indoor Office Channel Model," 2015 International Conference on Computing, Networking and Communications, ICNC 2015, pp. 26-31, Mar. 2015.

[42] Z. Yun and M. F. Iskander, "Ray Tracing for Radio Propagation Modeling: Principles and Applications," IEEE Access, vol. 3, pp. 1089-1100, Jul. 2015.

[43] A. Karstensen, W. Fan, I. Carton, and G. F. Pedersen, "Comparison of Ray Tracing Simulations and Channel Measurements at mmWave Bands for Indoor Scenarios," in 2016 10th European Conference on Antennas and Propagation (EuCAP), Apr. 2016, pp. 1-5.

[44] R. Heath, N. Gonzalez-Prelcic, S. Rangan, W. Roh, and A. Sayeed, "An Overview of Signal Processing Techniques for Millimeter Wave MIMO Systems," IEEE Journal of Selected Topics in Signal Processing, vol. 10, no. 3, pp. 436-453, Feb. 2016.

[45] F. Rusek, D. Persson, B. K. Lau, E. G. Larsson, T. L. Marzetta, O. Edfors, and F. Tufvesson, "Scaling Up MIMO: Opportunities and Challenges with Very Large Arrays," IEEE Signal Processing Magazine, vol. 30, no. 1, pp. 40-60, Jan. 2013.

[46] L. Lu, G. Y. Li, and A. L. Swindlehurst, "An Overview of Massive MIMO: Benefits and Challenges," IEEE Journal of Selected Topics in Signal Processing, vol. 8, no. 5, pp. 742-758, Oct. 2014.

[47] E. Björnson, J. Hoydis, M. Kountouris, and M. Debbah, "Massive MIMO Systems With Non-Ideal Hardware: Energy Efficiency, Estimation, and Capacity Limits," IEEE Transactions on Information Theory, vol. 60, no. 11, pp. 7112-7139, Nov. 2014.

[48] E. Björnson, E. G. Larsson, and T. L. Marzetta, "Massive MIMO: Ten Myths and One Critical Question," IEEE Communications Magazine, vol. 54, no. 2, pp. 114-123, Feb. 2016.

[49] H. Yang and T. L. Marzetta, "Massive MIMO in Line-of-Sight Propagation," in 2017 IEEE 85th Vehicular Technology Conference (VTC Spring), Jun. 2017, pp. 1-5.

[50] S. Sun, T. S. Rappaport, R. W. Heath, A. Nix, and S. Rangan, "MIMO for Millimeter-Wave Wireless Communications: Beamforming, Spatial Multiplexing, or Both?" IEEE Communications Magazine, vol. 52, no. 12, pp. 110-121, Dec. 2014. 
[51] S. Han, C. l. I, Z. Xu, and C. Rowell, "Large-scale Antenna Systems with Hybrid Analog and Digital Beamforming for Millimeter Wave 5G," IEEE Communications Magazine, vol. 53, no. 1, pp. 186-194, Jan. 2015.

[52] A. Alkhateeb, O. El Ayach, G. Leus, and R. W. Heath, "Channel Estimation and Hybrid Precoding for Millimeter Wave Cellular Systems," IEEE Journal on Selected Topics in Signal Processing, vol. 8, no. 5, pp. 831-846, Oct. 2014.

[53] A. Alkhateeb, G. Leus, and R. W. Heath, "Limited Feedback Hybrid Precoding for Multi-User Millimeter Wave Systems," IEEE Transactions on Wireless Communications, vol. 14, no. 11, pp. 6481-6494, Nov. 2015.

[54] H. Q. Ngo, E. G. Larsson, and T. L. Marzetta, "Energy and Spectral Efficiency of Very Large Multiuser MIMO Systems," IEEE Transactions on Communications, vol. 61, no. 4, pp. 1436-1449, Apr. 2013.

[55] O. E. Ayach, S. Rajagopal, S. Abu-Surra, Z. Pi, and R. W. Heath, "Spatially Sparse Precoding in Millimeter Wave MIMO Systems," IEEE Transactions on Wireless Communications, vol. 13, no. 3, pp. 1499-1513, Mar. 2014.

[56] R. R. Müller, L. Cottatellucci, and M. Vehkaperä, "Blind Pilot Decontamination," IEEE Journal of Selected Topics in Signal Processing, vol. 8, no. 5, pp. 773-786, Oct. 2014.

[57] E. Larsson, O. Edfors, F. Tufvesson, and T. Marzetta, "Massive MIMO for Next Generation Wireless Systems," IEEE Communications Magazine, vol. 52, no. 2, pp. 186-195, Feb. 2014.

[58] T. L. Marzetta, "Noncooperative Cellular Wireless with Unlimited Numbers of Base Station Antennas," IEEE Transactions on Wireless Communications, vol. 9, no. 11, pp. 3590-3600, Nov. 2010.

[59] O. Elijah, C. Y. Leow, T. A. Rahman, S. Nunoo, and S. Z. Iliya, "A Comprehensive Survey of Pilot Contamination in Massive MIMO-5G System," IEEE Communications Surveys Tutorials, vol. 18, no. 2, pp. 905-923, Nov. 2015.

[60] K. Appaiah, A. Ashikhmin, and T. L. Marzetta, "Pilot Contamination Reduction in Multi-User TDD Systems," in 2010 IEEE International Conference on Communications (ICC), May 2010, pp. 1-5. 
[61] H. Yin, D. Gesbert, M. C. Filippou, and Y. Liu, "Decontaminating Pilots in Massive MIMO Systems," in 2013 IEEE International Conference on Communications (ICC), Jun. 2013, pp. 3170-3175.

[62] J. Jose, A. Ashikhmin, T. L. Marzetta, and S. Vishwanath, "Pilot Contamination and Precoding in Multi-Cell TDD Systems," IEEE Transactions on Wireless Communications, vol. 10, no. 8, pp. 2640-2651, Aug. 2011.

[63] A. Ashikhmin and T. Marzetta, "Pilot Contamination Precoding in Multi-Cell Large Scale Antenna Systems," in 2012 IEEE International Symposium on Information Theory Proceedings (ISIT), Jul. 2012, pp. 1137-1141.

[64] B. D. Lakmali and D. Dias, "Database Correlation for GSM Location in Outdoor and Indoor Environments," Proceedings of the 2008 4th International Conference on Information and Automation for Sustainability, ICIAFS 2008, pp. 42-47, Dec. 2008.

[65] E. Kupershtein, M. Wax, and I. Cohen, "Single-Site Emitter Localization Via Multipath Fingerprinting," IEEE Transactions on Signal Processing, vol. 61, no. 1, pp. 10-21, Jan. 2013.

[66] V. Va, J. Choi, T. Shimizu, G. Bansal, and R. W. Heath, "Inverse Multipath Fingerprinting for Millimeter Wave V2I Beam Alignment," IEEE Transactions on Vehicular Technology, vol. 67, no. 5, pp. 4042-4058, May 2018.

[67] O. Simeone, A Brief Introduction to Machine Learning for Engineers. Now Publishers, 2018. [Online]. Available: http://arxiv.org/abs/1709. 02840

[68] M. G. Kibria, K. Nguyen, G. P. Villardi, O. Zhao, K. Ishizu, and F. Kojima, "Big Data Analytics, Machine Learning, and Artificial Intelligence in Next-Generation Wireless Networks," IEEE Access, vol. 6, pp. 32328 32 338, May 2018.

[69] K. Zheng, Z. Yang, K. Zhang, P. Chatzimisios, K. Yang, and W. Xiang, "Big Data-Driven Optimization for Mobile Networks Toward 5G," IEEE Network, vol. 30, no. 1, pp. 44-51, Jan. 2016.

[70] C. Jiang, H. Zhang, Y. Ren, Z. Han, K. Chen, and L. Hanzo, "Machine Learning Paradigms for Next-Generation Wireless Networks," IEEE Wireless Communications, vol. 24, no. 2, pp. 98-105, Apr. 2017. 
[71] A. Klautau, P. Batista, N. González-Prelcic, Y. Wang, and R. W. Heath, "5G MIMO Data for Machine Learning: Application to Beam-Selection using Deep Learning," 2018 Information Theory and Applications Workshop, Feb. 2018. [Online]. Available: http: //ita.ucsd.edu/workshop/18/files/paper/paper_3313.pdf

[72] Remcom, "Remcom Wireless Insite," https://www.remcom.com/ wireless-insite-em-propagation-software, accessed: 2019-05-02.

[73] Y. Wang, M. Narasimha, and R. W. Heath, "MmWave Beam Prediction with Situational Awareness: A Machine Learning Approach," in 2018 IEEE 19th International Workshop on Signal Processing Advances in Wireless Communications (SPAWC), Jun. 2018, pp. 1-5.

[74] Y. Wang, A. Klautau, M. Ribero, M. Narasimha, and R. W. Heath, "MmWave Vehicular Beam Training with Situational Awareness by Machine Learning," in 2018 IEEE Globecom Workshops (GC Wkshps), Dec. 2018, pp. 1-6.

[75] A. Mazin, M. Elkourdi, and R. D. Gitlin, "Accelerating Beam Sweeping in mmWave Standalone 5G New Radios Using Recurrent Neural Networks," in 2018 IEEE 88th Vehicular Technology Conference (VTC-Fall), Aug. 2018, pp. 1-4.

[76] A. Alkhateeb, I. Beltagy, and S. Alex, "Machine Learning for Reliable mmWave Systems: Blockage Prediction and Proactive Handoff," in 2018 IEEE Global Conference on Signal and Information Processing (GlobalSIP), Nov. 2018, pp. 1055-1059.

[77] A. Alkhateeb, S. Alex, P. Varkey, Y. Li, Q. Qu, and D. Tujkovic, "Deep Learning Coordinated Beamforming for Highly-Mobile Millimeter Wave Systems," IEEE Access, vol. 6, pp. 1-43, Jun. 2018.

[78] V. Va, T. Shimizu, G. Bansal, and R. W. Heath, "Online Learning for Position-Aided Millimeter Wave Beam Training," IEEE Access, vol. 7, pp. 30 507-30 526, May 2019.

[79] C. Antón-Haro and X. Mestre, "Learning and Data-Driven Beam Selection for mmWave Communications: An Angle of Arrival-Based Approach," IEEE Access, vol. 7, pp. 20 404-20 415, Jan. 2019.

[80] A. Alkhateeb, "DeepMIMO: A Generic Deep Learning Dataset for Millimeter Wave and Massive MIMO Applications," http://arxiv.org/abs/ 1902.06435, pp. 1-8, Feb. 2019. 
[81] National Institute of Standards and Technology (NIST), "5G mmWave Channel Model Alliance," https:// www.nist.gov/communications-technology-laboratory/ 5g-mmwave-channel-model-alliance, accessed: 2019-06-02.

[82] DeepSIG, "RF Datasets for Machine Learning," https://www.deepsig.io/ datasets, accessed: 2019-06-02.

[83] GNU Radio, "The GNU Radio Signal Metadata Format Specification," https://github.com/gnuradio/SigMF, accessed: 2019-06-02.

[84] 3GPP TSG RAN, "NR; Physical Channels and Modulation," 3rd Generation Partnership Project (3GPP), Technical Specification (TS) 38.211, Jun. 2018, version 15.2.0.

[85] A. A. Zaidi, R. Baldemair, V. Moles-Cases, N. He, K. Werner, and A. Cedergren, "OFDM Numerology Design for 5G New Radio to Support IoT, eMBB, and MBSFN," IEEE Communications Standards Magazine, vol. 2, no. 2, pp. 78-83, Jun. 2018.

[86] 3GPP TSG RAN, "NR; Physical Layer Procedures For Control," 3rd Generation Partnership Project (3GPP), Technical Specification (TS) 38.213, Jun. 2018, version 15.2.0.

[87] 3GPP TSG RAN, "NR; Radio Resource Control (RRC); Protocol specification," 3rd Generation Partnership Project (3GPP), Technical Specification (TS) 38.331, Jun. 2018, version 15.2.0.

[88] 3GPP TSG RAN, "Study on Elevation Beamforming/Full-Dimension (FD) MIMO for LTE," 3rd Generation Partnership Project (3GPP), Technical Specification (TS) 36.897, Jul. 2015, version 13.0.0.

[89] 3GPP TSG RAN, "NR; Physical Layer Procedures For Data," 3rd Generation Partnership Project (3GPP), Technical Specification (TS) 38.214, 2018, version 15.2.0.

[90] 3GPP TSG RAN, "NR; Physical Layer Measurements," 3rd Generation Partnership Project (3GPP), TS 38.215, Jun. 2018, version 15.2.0.

[91] 3GPP TSG RAN, "Study on New Radio Access Technology Physical Layer Aspects," 3rd Generation Partnership Project (3GPP), Technical Report (TR) 38.802, Dec. 2017, version 15.0.0.

[92] A. M. Sayeed, "Deconstructing Multiantenna Fading Channels," IEEE Transactions on Signal Processing, vol. 50, no. 10, pp. 2563-2579, Oct. 2002. 
[93] A. Alkhateeb, J. Mo, N. González-Prelcic, and R. W. Heath, "MIMO Precoding and Combining Solutions for Millimeter-Wave Systems," IEEE Communications Magazine, vol. 52, no. 12, pp. 122-131, Dec. 2014.

[94] A. A. M. Saleh and R. Valenzuela, "A Statistical Model for Indoor Multipath Propagation," IEEE Journal on Selected Areas in Communications, vol. 5, no. 2, pp. 128-137, Feb. 1987.

[95] Y. Zhao, C. Yan, X. Zhou, Q. Zhu, S. Wang, and W. Guo, "The Research and Development of 3D Urban Geographic Information System with Unity3D," in 2013 21st International Conference on Geoinformatics, Jun. 2013, pp. 1-4.

[96] "OpenStreetMap (OSM)," https://www.openstreetmap.org, accessed: 2019-07-02.

[97] D. Wilkie, J. Sewall, and M. C. Lin, "Transforming GIS Data into Functional Road Models for Large-Scale Traffic Simulation," IEEE Transactions on Visualization and Computer Graphics, vol. 18, no. 6, pp. 890901, Jun. 2012.

[98] M. Over, A. Schilling, S. Neubauer, and A. Zipf, "Generating Web-Based 3D City Models from OpenStreetMap: The Current Situation in Germany," Computers, Environment and Urban Systems, vol. 34, no. 6, pp. 496-507, Nov. 2010.

[99] S. Hussain, "Efficient Ray-Tracing Algorithms for Radio Wave Propagation in Urban Environments," Ph.D. dissertation, University of the City of Dublin, 2017.

[100] ITU-R, "Calculation of Free-Space Attenuation," International Telecommunication Union (ITU), P 525-2, Aug. 2019.

[101] D. Yang, L. . Yang, and L. Hanzo, "DFT-Based Beamforming WeightVector Codebook Design for Spatially Correlated Channels in the Unitary Precoding Aided Multiuser Downlink," in 2010 IEEE International Conference on Communications, May 2010, pp. 1-5.

[102] J. Li, X. Su, J. Zeng, Y. Zhao, S. Yu, L. Xiao, and X. Xu, "Codebook Design for Uniform Rectangular Arrays of Massive Antennas," in 2013 IEEE r7th Vehicular Technology Conference (VTC Spring), Jun. 2013, pp. $1-5$. 
[103] Y. Xie, S. Jin, J. Wang, Y. Zhu, X. Gao, and Y. Huang, "A Limited Feedback Scheme For 3D Multiuser MIMO based on Kronecker Product Codebook," in 2013 IEEE 24 th Annual International Symposium on Personal, Indoor, and Mobile Radio Communications (PIMRC), Sep. 2013, pp. 1130-1135.

[104] M. Mezzavilla, M. Zhang, M. Polese, R. Ford, S. Dutta, S. Rangan, and M. Zorzi, "End-to-End Simulation of 5G mmWave Networks," IEEE Communications Surveys Tutorials, vol. 20, no. 3, pp. 2237-2263, Apr. 2018.

[105] M. Zhang, M. Polese, M. Mezzavilla, S. Rangan, and M. Zorzi, "Ns3 Implementation of the 3GPP MIMO Channel Model for Frequency Spectrum Above $6 \mathrm{GHz}$," in Proceedings of the Workshop on Ns-3, ser. WNS3 '17. ACM, Jun. 2017, pp. 71-78.

[106] C. Herranz. (2018) Extension of the mmWave ns-3 Module Enabling Beam Management with 3GPP NR SSB and CSI-RS Measurements. [Online]. Available: https://github.com/carhercl/ mmWave-beam-management-ns- 3.26

[107] M. Mezzavilla, S. Dutta, M. Zhang, M. R. Akdeniz, and S. Rangan, "5G MmWave Module for the Ns-3 Network Simulator," in Proceedings of the 18th ACM International Conference on Modeling, Analysis and Simulation of Wireless and Mobile Systems, Nov. 2015, pp. 283-290.

[108] S. Ha, I. Rhee, and L. Xu, "CUBIC: A New TCP-friendly High-speed TCP Variant," SIGOPS Oper. Syst. Rev., vol. 42, no. 5, pp. 64-74, Jul. 2008.

[109] D. Martín-Sacristán, C. Herranz, J. F. Monserrat, A. Szczygiel, N. P. Kuruvatti, D. Garcia-Roger, D. Prado, M. Boldi, J. Belschner, and H. D. Schotten, "5G Visualization: The METIS-II Project Approach," Mobile Information Systems, vol. 2018, p. 8, Sep. 2018.

[110] V. M. N. Passaro, A. Cuccovillo, L. Vaiani, M. D. Carlo, and C. E. Campanella, "Gyroscope Technology and Applications: A Review in the Industrial Perspective," Sensors, vol. 17, Oct. 2017.

[111] C. J. Fisher, "Using an Accelerometer for Inclination Sensing," Analog Devices, AN- 1057, 2010. [Online]. Available: https://www.analog.com/ media/en/technical-documentation/application-notes/AN-1057.pdf 
[112] J. D. Weiss and F. Shields, "GPS/INS Integration in a Severe Urban Environment," in IEEE 1998 Position Location and Navigation Symposium (Cat. No.98CH36153), Apr. 1998, pp. 432-440.

[113] G. De Angelis, G. Baruffa, and S. Cacopardi, "GNSS/Cellular Hybrid Positioning System for Mobile Users in Urban Scenarios," IEEE Transactions on Intelligent Transportation Systems, vol. 14, no. 1, pp. 313-321, Mar. 2013.

[114] The Office of the Secretary of Defense, "GPS Standard Positioning Service (SPS) Performance Standard," U.S. Government, Tech. Rep., Sep. 2008. [Online]. Available: https://www.gps.gov/technical/ps/ 2008-SPS-performance-standard.pdf

[115] B. Ben-Moshe, E. Elkin, H. Levi, and A. Weissman, "Improving Accuracy of GNSS Devices in Urban Canyons," in 23rd Annual Canadian Conference on Computational Geometry, Aug. 2011.

[116] K. Kira and L. A. Rendell, "A Practical Approach to Feature Selection," in Proceedings of the Ninth International Workshop on Machine Learning, Jul. 1992, pp. 249-256.

[117] A. Zheng and A. Casari, Feature Engineering for Machine Learning: Principles and Techniques for Data Scientists, 1st ed. O'Reilly Media Inc., 2018.

[118] V. Nair and G. E. Hinton, "Rectified Linear Units Improve Restricted Boltzmann Machines," in Proceedings of the 27th International Conference on International Conference on Machine Learning, Jun. 2010, pp. 807-814.

[119] S. Amari, "Backpropagation and Stochastic Gradient Descent Method," Neurocomputing, vol. 5, no. 4, pp. 185-196, Jun. 1993.

[120] I. Goodfellow, Y. Bengio, and A. Courville, Deep Learning. MIT Press, 2016, http://www.deeplearningbook.org.

[121] S. Hur, T. Kim, D. J. Love, J. V. Krogmeier, T. A. Thomas, and A. Ghosh, "Millimeter Wave Beamforming for Wireless Backhaul and Access in Small Cell Networks," IEEE Transactions on Communications, vol. 61, no. 10, pp. 4391-4403, Oct. 2013.

[122] Z. Xiao, T. He, P. Xia, and X. Xia, "Hierarchical Codebook Design for Beamforming Training in Millimeter-Wave Communication," IEEE 
Transactions on Wireless Communications, vol. 15, no. 5, pp. 3380-3392, May 2016.

[123] P. Marsch, Ö. Bulakci, O. Queseth, and M. Boldi, 5G System Design: Architectural and Functional Considerations and Long Term Research. Wiley, 2018.

[124] S. Gimenez, S. Roger, D. Martín-Sacristán, J. F. Monserrat, P. Baracca, V. Braun, and H. Halbauer, "Performance of Hybrid Beamforming for mmW Multi-Antenna Systems in Dense Urban Scenarios," in 2016 IEEE 27th Annual International Symposium on Personal, Indoor, and Mobile Radio Communications (PIMRC), Sep. 2016, pp. 1-6.

[125] Networld2020 and 5GIA, "Strategic Research and Innovation Agenda 2021-27. Smart Networks in the context of NGI," Networld2020, Tech. Rep., Sep. 2018. [Online]. Available: https://www.networld2020.eu/ wp-content/uploads/2018/11/networld2020-5gia-sria-version-2.0.pdf

[126] O. Ozgun, "New Software Tool (GO+UTD) for Visualization of Wave Propagation [Testing Ourselves]," IEEE Antennas and Propagation Magazine, vol. 58, no. 3, pp. 91-103, Jun. 2016.

[127] G. E. Marai, A. G. Forbes, and A. Johnson, "Interdisciplinary Immersive Analytics at the Electronic Visualization Laboratory: Lessons Learned and Upcoming Challenges," in 2016 Workshop on Immersive Analytics (IA), Mar. 2016, pp. 54-59.

[128] N. Gershon and W. Page, "What Storytelling Can Do for Information Visualization," ACM Commununications, vol. 44, no. 8, pp. 31-37, Aug. 2001.

[129] M. Zyda, "From Visual Simulation to Virtual Reality to Games," Computer, vol. 38, no. 9, pp. 25-32, Sep. 2005.

[130] R. Brath, M. Peters, and R. Senior, "Visualization for Communication: the Importance of Aesthetic Sizzle," in 9th International Conference on Information Visualisation (IV'05), Jul. 2005, pp. 724-729.

[131] A. Menin, R. Torchelsen, and L. Nedel, "An Analysis of VR Technology Used in Immersive Simulations with a Serious Game Perspective," IEEE Computer Graphics and Applications, vol. 38, no. 2, pp. 57-73, Mar. 2018. 
[132] A. Navarro, J. V. Pradilla, and P. Madriñán, "A 3D Game Tool for Mobile Networks Planning," in 2010 Second International Conference on Mobile, Hybrid, and On-Line Learning, Feb. 2010, pp. 158-161.

[133] P. Chakraborty, N. P. Kuruvatti, and H. D. Schotten, "A Novel Serious Game Engineering Based Interactive Visualization and Evaluation Platform for Cellular Technologies," in 2017 International Symposium on Networks, Computers and Communications (ISNCC), May 2017, pp. $1-6$.

[134] D. Martín-Sacristán, C. Herranz, and J. F. Monserrat, "Traffic Safety in the METIS-II 5G Connected Cars Use Case: Technology Enablers and Baseline Evaluation," in EuCNC 2017 - European Conference on Networks and Communications, Jun. 2017, pp. 1-5.

[135] G. Huang, J. Belschner, A. Szczygiel, M. Boldi, D. Garcia-Roger, C. Herranz, D. Martín-Sacristán, J. F. Monserrat, D. Prado, N. P. Kuruvatti, and J. Lianghai, "Final 5G Visualization," METIS-II, Deliverable D7.3, Jun. 2017.

[136] C. Johnson, "Top Scientific Visualization Research Problems," IEEE Computer Graphics and Applications, vol. 24, no. 4, pp. 13-17, Jul. 2004.

[137] B. Lee, N. H. Riche, P. Isenberg, and S. Carpendale, "More Than Telling a Story: Transforming Data into Visually Shared Stories," IEEE Computer Graphics and Applications, vol. 35, no. 5, pp. 84-90, Sep. 2015.

[138] P. Agyapong, V. Braun, M. Fallgren, S. Jeux, A. Klein, J. Lianghai, D. Martín-Sacristán, M. Maternia, M. Moisio, J. F. Monserrat, K. Pawlak, H. Tullberg, and A. Weber, "Simulation Guidelines," METISI, Deliverable D6.1, Oct. 2013.

[139] J. S. Yi, Y. a. Kang, and J. Stasko, "Toward a Deeper Understanding of the Role of Interaction in Information Visualization," IEEE Transactions on Visualization and Computer Graphics, vol. 13, no. 6, pp. 1224-1231, Nov. 2007. 